\title{
Visible-Light-Driven Selective Alkenyl C-P Bond Cleavage of Allenylphosphine Oxides
}

\author{
Kai Wei, ${ }^{\dagger,}$ Kai Luo, ${ }^{\dagger, \S}$ Fang Liu, ${ }^{\dagger}$ Lei Wu, ${ }^{*, \dagger}$ and Li-Zhu Wu* ${ }^{*}$ \\ †Jiangsu Key Laboratory of Pesticide Science and Department of Chemistry, College of Sciences, \\ Nanjing Agricultural University, Nanjing 210095, China \\ *Key Laboratory of Photochemical Conversion and Optoelectronic Materials, Technical Institute of \\ Physics and Chemistry, Chinese Academy of Sciences, Beijing, P. R. China
}

Table of Contents for Supporting Information

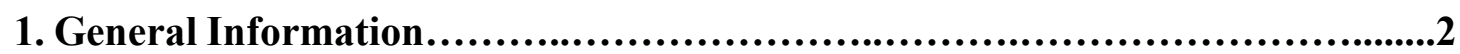

2. General Preparation for Substrates Preparation and Characterizations..............2

3. General Procedures for Visible-Light-Driven C-P Cleavage and Migration of Allenylphosphine Oxide.........................................................12

4. Optimization of the Reaction Conditions and the Reactions of Single Electron Oxidants..................................................................13

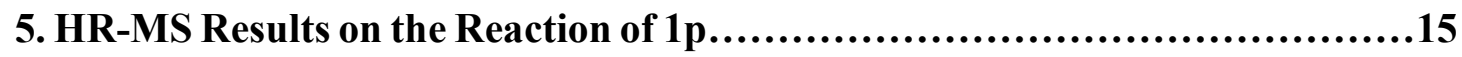

6. Crossover Experiment...........................................................................16

7. ${ }^{18}$ O-labeling Experiment...........................................................................16

8. Determination of Singlet Oxygen Species............................................................18

9. Quenching Experiments of Erythrosin B............................................................21

10. The Reaction Rate Curves With or Without of BI-OH.......................21

11. X-Ray Crystallography Data of Compound 2b.............................22

12. Characterizations of Products..................................................31

13. ${ }^{1} \mathrm{H} /{ }^{13} \mathrm{C} /{ }^{31} \mathrm{P}-\mathrm{NMR}$, and HRMS Spectra of Substrates and Products.....................44 


\section{General Information:}

Solvents and reagents were reagent grade and used without purification unless otherwise noted. Column chromatography was performed using silica gel (200-300 mesh). All ${ }^{1} \mathrm{H}-\mathrm{NMR}$ (400 MHz) spectra were recorded on a Bruker-DMX 400 using $\mathrm{CDCl}_{3}$ solution in the presence of tetramethylsilane (TMS) as an internal standard and are reported in ppm $(\delta)$. Coupling constants are reported in Hertz (Hz). Spectral splitting patterns are designated as s, singlet; d, doublet; t, triplet; q, quartet; p, pentet; m, multiplet; and br, broad. High resolution mass spectroscopic data of the products were collected on a Waters Micromass GCT instrument using EI $(70 \mathrm{eV})$ or an Agilent Technologies 6540 UHD Accurate-Mass Q-TOF LC/MS using ESI.

\section{General Procedures for Substrates Preparation and Characterizations:}

\subsection{Substrates Preparation}

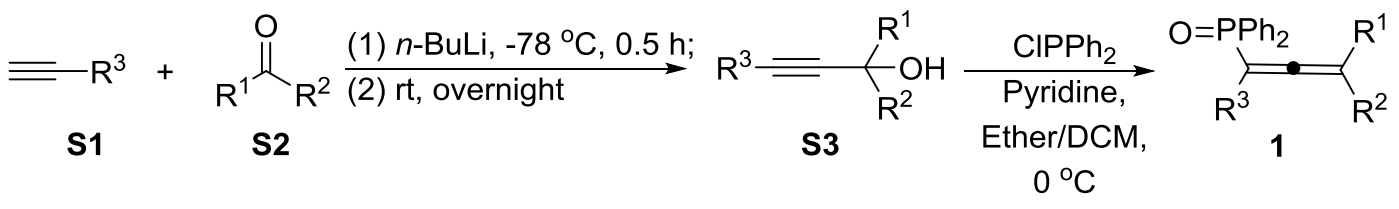

To a solution of terminal alkyne $(\mathbf{S 1}, 40 \mathrm{mmol})$ in anhydrous THF $(40 \mathrm{~mL})$ was added $n$-BuLi (2.5 $\mathrm{M}$ in hexane, $48 \mathrm{mmol}$ ) drop wisely at $-78^{\circ} \mathrm{C}$. The mixture was stirred for 30 minutes and then $50 \mathrm{mmol}$ of ketone (S2) was added, which was maintained at $-78^{\circ} \mathrm{C}$ for $1 \mathrm{~h}$ before warm-up. After 12 hours, the reaction was quenched with $50 \mathrm{~mL}$ saturated $\mathrm{NH}_{4} \mathrm{Cl}$ solution, extracted with ethyl acetate, and dried with anhydrous $\mathrm{Na}_{2} \mathrm{SO}_{4}$. The resulting acetylenic alcohol (S3) could be used directly after concentration. The procedures were modified from reported works: a) Hashimi, A. S. K.; Wang, T.; Shi, S.; Rudolph, M. J. Org. Chem. 2012, 77, 7761-7767. b) Liu, P.; Deng, C.-L.; Lei, X.; Lin, G. Eur. J. Org. Chem. 2011, 7308-7316.

To a solution of the acetylenic alcohol $(\mathbf{S 3}, 40 \mathrm{mmol})$ in anhydrous ethyl ether $(50 \mathrm{~mL})$ and pyridine $(3.88 \mathrm{~mL}, 48 \mathrm{mmol})$ under nitrogen was added dropwise a solution of diphenylphosphine chloride (40 mmol) in $\mathrm{CH}_{2} \mathrm{Cl}_{2}(50 \mathrm{~mL})$. After 12 hours, the reaction was quenched with water and extracted with $\mathrm{CH}_{2} \mathrm{Cl}_{2}$ for three times, and the combined organic layer was dried with $\mathrm{Na}_{2} \mathrm{SO}_{4}$. The crude product was subjected to flash chromatography on silica gel eluting with ethyl acetate and petroleum ether (v:v=1:1) to afford the purified allenylphosphine oxides (1).

\section{[NOTE]:}


The relative lower yields $(<50 \%)$ of some substrates were due to the slow reaction rate in the second step of preparation, and the unreacted compound $\mathbf{S 3}$ could be easily recovered during the isolation of compound $\mathbf{1 .}$

Readers may consider that the propargylic phosphate (2, products of this paper) can be produced while preparing the allenylphosphine oxides (1). However, as shown in the following TLC figures, control experiments clearly ruled out the possibility.

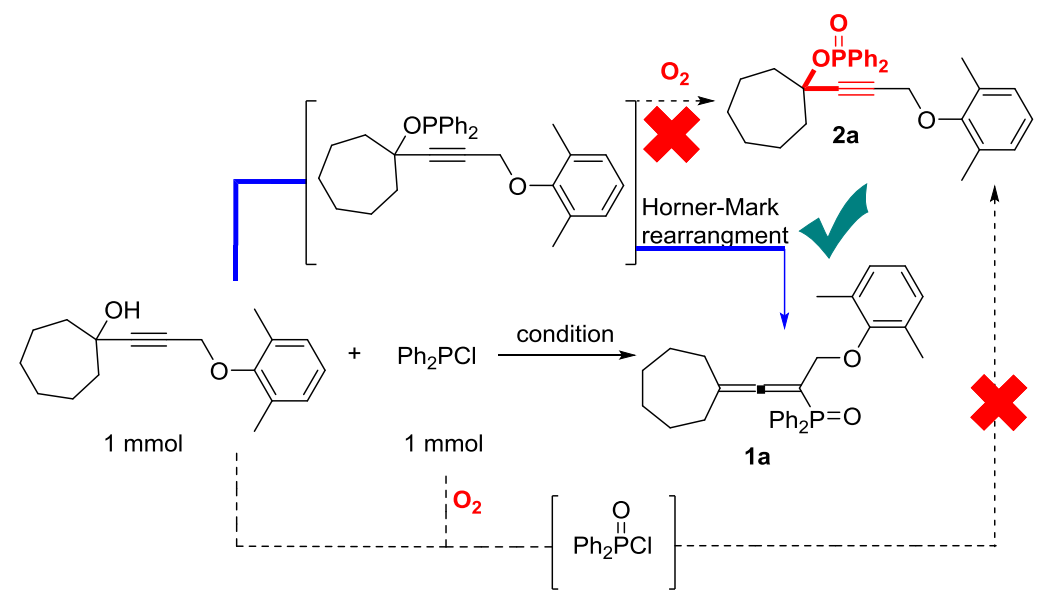

Condition A: pyridine, ether, $0^{\circ} \mathrm{C}$ to $\mathrm{rt}, 12 \mathrm{~h}, \mathrm{~N}_{2}$; only $1 \mathrm{a}$ !!!

Condition B: pyridine, ether, $0^{\circ} \mathrm{C}$ to rt, $12 \mathrm{~h}, \mathrm{O}_{2}$ balloon; only $1 \mathrm{a} ! ! !(<10 \%)$

acetate : petroleum ether $=1: 2$
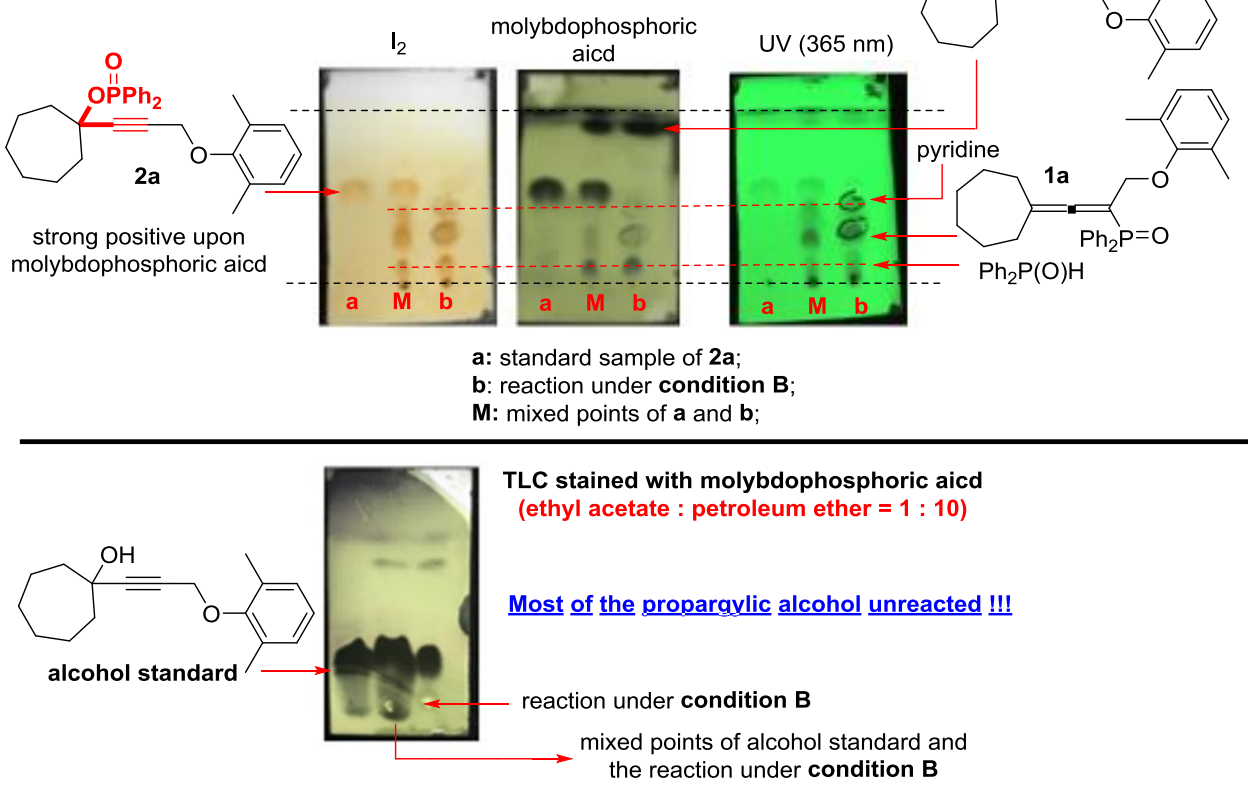

The standard condition under nitrogen gave no product of propargylic phosphate (2a). A parallel experiment equipped with oxygen balloon turned to be sluggish, with large amount of propargylic alcohol left. 


\subsection{Substrates Characterizations}

For known compounds: $(\mathbf{1 a}, \mathbf{1 n}, \mathbf{1 p}),{ }^{1}(\mathbf{1 b}, \mathbf{1 g}, \mathbf{1 r}),{ }^{2}(\mathbf{1 c}, \mathbf{1 d}, \mathbf{1 f}, \mathbf{1 t}, \mathbf{1 v}, \mathbf{1 w}, \mathbf{1 a a}),{ }^{3}(\mathbf{1 e}$, 1o, 1x, 1y), ${ }^{4}$ (1h, 1i, 1j, 1m,1k), ${ }^{5}(\mathbf{1 z}, \mathbf{1 a b}, \mathbf{1 a e}, \mathbf{1 a h}, 1 \mathrm{ai}){ }^{6}$

(1-(dihydrothiophen-3(2H)-ylidene)-3-(2,6-dimethylphenoxy)prop-1-en-2-yl)diphenylphosphine oxide (1d)<smiles>Cc1cccc(C)c1OCC(=CC1CCSC1)P=O</smiles>

A white solid. Yield: $45 \% .{ }^{1} \mathbf{H}$ NMR $\left(400 \mathrm{MHz}, \mathrm{CDCl}_{3}\right) \delta 7.85-7.73(\mathrm{~m}, 4 \mathrm{H}), 7.56-7.52(\mathrm{~m}, 2 \mathrm{H})$, $7.51-7.45(\mathrm{~m}, 4 \mathrm{H}), 6.97-6.85(\mathrm{~m}, 3 \mathrm{H}), 4.54(\mathrm{~d}, J=8.3 \mathrm{~Hz}, 2 \mathrm{H}), 3.60-3.51(\mathrm{~m}, 1 \mathrm{H}), 3.22-3.11$ (m, 1H), $2.81-2.64(\mathrm{~m}, 2 \mathrm{H}), 2.46-2.35(\mathrm{~m}, 2 \mathrm{H}), 2.16(\mathrm{~s}, 6 \mathrm{H}) .{ }^{13} \mathbf{C}$ NMR $\left(101 \mathrm{MHz}, \mathrm{CDCl}_{3}\right) \delta$ $203.6(\mathrm{~d}, J=5.2 \mathrm{~Hz}), 155.4,132.5(\mathrm{~d}, J=8.1 \mathrm{~Hz}), 132.1(\mathrm{~d}, J=3.0 \mathrm{~Hz}), 132.0(\mathrm{~d}, J=3.0 \mathrm{~Hz}), 131.6$ $(\mathrm{d}, J=10.1 \mathrm{~Hz}), 131.4(\mathrm{~d}, J=7.1 \mathrm{~Hz}), 130.9,128.8,128.5(\mathrm{~d}, J=4.6 \mathrm{~Hz}), 128.4(\mathrm{~d}, J=5.1 \mathrm{~Hz})$, 124.1, $106.4(\mathrm{~d}, J=13.7 \mathrm{~Hz}), 99.3,98.3,67.6(\mathrm{~d}, J=12.5 \mathrm{~Hz}), 35.0(\mathrm{~d}, J=5.0 \mathrm{~Hz}), 32.7(\mathrm{~d}, J=5.6$ Hz), $31.9(\mathrm{~d}, J=2.2 \mathrm{~Hz}), 16.3 .{ }^{31} \mathbf{P}$ NMR $\left(162 \mathrm{MHz}, \mathrm{CDCl}_{3}\right) \delta 29.48$ (s). HRMS (ESI): $\left([\mathrm{M}+\mathrm{Na}]^{+}\right)$ Calcd for $\mathrm{C}_{27} \mathrm{H}_{27} \mathrm{NaO}_{2} \mathrm{PS}$ : 469.1367, Found: 469.1361 .

(1-(dihydro-2H-thiopyran-4(3H)-ylidene)-3-(2,6-dimethylphenoxy)prop-1-en-2-yl)diphenyl phosphine oxide (1e)<smiles>Cc1cccc(C)c1OCC(=CC1CCSCC1)[PH](=O)P</smiles>

A white solid. Yield: $56 \% .{ }^{1} \mathbf{H}$ NMR $\left(400 \mathrm{MHz}, \mathrm{CDCl}_{3}\right) \delta 7.87-7.75(\mathrm{~m}, 4 \mathrm{H}), 7.61-7.45(\mathrm{~m}, 6 \mathrm{H})$, $6.99-6.84(\mathrm{~m}, 3 \mathrm{H}), 4.49(\mathrm{~d}, J=8.6 \mathrm{~Hz}, 2 \mathrm{H}), 2.57-2.46(\mathrm{~m}, 2 \mathrm{H}), 2.43-2.33(\mathrm{~m}, 2 \mathrm{H}), 2.31-2.23$ (m, 2H), $2.17(\mathrm{~d}, J=3.0 \mathrm{~Hz}, 1 \mathrm{H}), 2.14(\mathrm{~s}, 6 \mathrm{H}), 2.11(\mathrm{~d}, J=3.2 \mathrm{~Hz}, 1 \mathrm{H}) .{ }^{13} \mathbf{C}$ NMR $(101 \mathrm{MHz}$, $\left.\mathrm{CDCl}_{3}\right) \delta 206.5(\mathrm{~d}, J=6.0 \mathrm{~Hz}), 155.4,132.6,131.9(\mathrm{~d}, J=2.0 \mathrm{~Hz}), 131.9,131.8,131.5,131.0$, 128.8, 128.5, 128.4, 124.1, $103.6(\mathrm{~d}, J=13.2 \mathrm{~Hz}), 96.2,95.2,67.7(\mathrm{~d}, J=12.6 \mathrm{~Hz}), 31.1$ (d, $J=5.3$ Hz), $28.7(\mathrm{~d}, J=3.7 \mathrm{~Hz}), 16.3 .{ }^{31} \mathbf{P}$ NMR $\left(162 \mathrm{MHz}, \mathrm{CDCl}_{3}\right) \delta 29.76$ (s). HRMS (ESI): $\left([\mathrm{M}+\mathrm{Na}]^{+}\right)$ Calcd for $\mathrm{C}_{28} \mathrm{H}_{29} \mathrm{NaO}_{2} \mathrm{PS}: 483.1524$, Found: 483.1518 . 
(1-(dihydro-2H-pyran-4(3H)-ylidene)-3-(2,6-dimethylphenoxy)prop-1-en-2-yl)diphenylphosphine oxide (1f)<smiles>Cc1cccc(C)c1OCC(=P)P=O</smiles>

A white solid. Yield: $62 \% .{ }^{1} \mathbf{H}$ NMR $\left(400 \mathrm{MHz}, \mathrm{CDCl}_{3}\right) \delta 7.86-7.76(\mathrm{~m}, 4 \mathrm{H}), 7.57-7.52(\mathrm{~m}, 2 \mathrm{H})$, $7.51-7.45(\mathrm{~m}, 4 \mathrm{H}), 6.98-6.84(\mathrm{~m}, 3 \mathrm{H}), 4.51(\mathrm{~d}, J=8.7 \mathrm{~Hz}, 2 \mathrm{H}), 3.69-3.58(\mathrm{~m}, 2 \mathrm{H}), 3.12-3.03$ (m, 2H), $2.30-2.25(\mathrm{~m}, 1 \mathrm{H}), 2.23-2.19(\mathrm{~m}, 1 \mathrm{H}), 2.15(\mathrm{~s}, 6 \mathrm{H}), 2.02-1.93(\mathrm{~m}, 2 \mathrm{H}) .{ }^{13} \mathbf{C}$ NMR $\left(101 \mathrm{MHz}, \mathrm{CDCl}_{3}\right) \delta 206.0(\mathrm{~d}, J=5.9 \mathrm{~Hz}), 155.4,132.7,132.0(\mathrm{~d}, J=3.0 \mathrm{~Hz}), 131.9,131.8,131.6$, 131.0, 128.8, 128.5, 128.3, 124.1, $101.4(\mathrm{~d}, J=13.3 \mathrm{~Hz}), 96.7,95.7,67.8(\mathrm{~d}, J=13.1 \mathrm{~Hz}), 67.5(\mathrm{~d}$, $J=3.0 \mathrm{~Hz}), 29.8(\mathrm{~d}, J=5.1 \mathrm{~Hz}), 16.3 .{ }^{31} \mathbf{P}$ NMR $\left(162 \mathrm{MHz}, \mathrm{CDCl}_{3}\right) \delta 29.96(\mathrm{~s})$. HRMS (ESI): $\left([\mathrm{M}+\mathrm{Na}]^{+}\right)$Calcd for $\mathrm{C}_{28} \mathrm{H}_{29} \mathrm{NaO}_{3} \mathrm{P}: 467.1752$, Found: 467.1746.

(1-(2,6-dimethylphenoxy)-4-methyldeca-2,3-dien-2-yl)diphenylphosphine oxide (1i)<smiles>CCCCCC/C(C)=C/C(COc1c(C)cccc1C)[PH](=O)P</smiles>

Pale yellow oil. Yield: $42 \% .{ }^{1} \mathbf{H}$ NMR $\left(400 \mathrm{MHz}, \mathrm{CDCl}_{3}\right) \delta 7.85-7.77(\mathrm{~m}, 4 \mathrm{H}), 7.54-7.49(\mathrm{~m}$, 2H), $7.48-7.41$ (m, 4H), $6.98-6.83(\mathrm{~m}, 3 \mathrm{H}), 4.48(\mathrm{~d}, J=9.3 \mathrm{~Hz}, 2 \mathrm{H}), 2.15$ (s, 6H), $1.83-1.66$ (m, 2H), $1.55(\mathrm{~d}, J=5.9 \mathrm{~Hz}, 3 \mathrm{H}), 1.24-1.05(\mathrm{~m}, 8 \mathrm{H}), 0.87(\mathrm{t}, J=7.0 \mathrm{~Hz}, 3 \mathrm{H}) .{ }^{13} \mathbf{C}$ NMR $(101$ $\left.\mathrm{MHz}, \mathrm{CDCl}_{3}\right) \delta 209.4(\mathrm{~d}, J=6.0 \mathrm{~Hz}), 155.4,132.9$ (d, $\left.J=32.4 \mathrm{~Hz}\right), 132.1,131.8(\mathrm{~d}, J=14.1 \mathrm{~Hz})$, $131.8(\mathrm{~d}, J=2.0 \mathrm{~Hz}), 131.7(\mathrm{~d}, J=5.1 \mathrm{~Hz}), 131.1,128.7,128.2(\mathrm{~d}, J=8.1 \mathrm{~Hz}), 128.1(\mathrm{~d}, J=8.1$ $\mathrm{Hz}), 124.0,104.0$ (d, $J=13.4 \mathrm{~Hz}), 95.8,94.7,68.1$ (d, $J=12.5 \mathrm{~Hz}), 33.1$ (d, $J=5.1 \mathrm{~Hz}), 31.6,28.9$, $27.1(\mathrm{~d}, J=2.2 \mathrm{~Hz}), 22.5,17.7(\mathrm{~d}, J=5.6 \mathrm{~Hz}), 16.2,14.1 .{ }^{31} \mathbf{P}$ NMR $\left(162 \mathrm{MHz}, \mathrm{CDCl}_{3}\right) \delta 30.10(\mathrm{~s})$.

(1-(2,6-dimethylphenoxy)-4,5-dimethylhexa-2,3-dien-2-yl)diphenylphosphine oxide (1j)<smiles>Cc1cccc(C)c1OCC(=CC(C)C)P=O</smiles>

Pale yellow oil. Yield: $35 \% .{ }^{1} \mathbf{H}$ NMR $\left(400 \mathrm{MHz}, \mathrm{CDCl}_{3}\right) \delta 7.89-7.75(\mathrm{~m}, 4 \mathrm{H}), 7.55-7.39(\mathrm{~m}$, 6H), $6.99-6.83(\mathrm{~m}, 3 \mathrm{H}), 4.53-4.42(\mathrm{~m}, 2 \mathrm{H}), 2.14(\mathrm{~s}, 6 \mathrm{H}), 2.05-1.94(\mathrm{~m}, 1 \mathrm{H}), 1.60(\mathrm{~d}, J=5.8$ 
$\mathrm{Hz}, 3 \mathrm{H}), 0.86(\mathrm{~d}, J=6.8 \mathrm{~Hz}, 3 \mathrm{H}), 0.68(\mathrm{~d}, J=6.8 \mathrm{~Hz}, 3 \mathrm{H}) .{ }^{13} \mathbf{C} \mathbf{N M R}\left(101 \mathrm{MHz}, \mathrm{CDCl}_{3}\right) \delta 208.7$ (d, $J=6.5 \mathrm{~Hz}), 155.4,133.3,132.5(\mathrm{~d}, J=45.5 \mathrm{~Hz}), 132.0(\mathrm{~d}, J=10.1 \mathrm{~Hz}), 132.8(\mathrm{~d}, J=8.1 \mathrm{~Hz})$, 131.7 (d, $J=8.1 \mathrm{~Hz}), 131.1,128.7,128.3$ (d, $J=9.1 \mathrm{~Hz}), 128.2$ (d, $J=8.1 \mathrm{~Hz}), 124.0,109.4$ (d, $J$ $=13.3 \mathrm{~Hz}), 96.8,95.8,68.0(\mathrm{~d}, J=12.6 \mathrm{~Hz}), 31.7$ (d, $J=4.8 \mathrm{~Hz}), 21.0$ (d, $J=2.1 \mathrm{~Hz}), 20.5$ (d, $J=$ $2.9 \mathrm{~Hz}), 16.1,15.9$ (d, $J=5.6 \mathrm{~Hz}) .{ }^{31} \mathbf{P}$ NMR $\left(162 \mathrm{MHz}, \mathrm{CDCl}_{3}\right) \delta 30.07$ (s). HRMS (ESI): $\left([\mathrm{M}+\mathrm{Na}]^{+}\right)$Calcd for $\mathrm{C}_{28} \mathrm{H}_{31} \mathrm{NaO}_{2} \mathrm{P}: 453.1959$, Found: 453.1953 .

(4-cyclohexyl-1-(2,6-dimethylphenoxy)penta-2,3-dien-2-yl)diphenylphosphine oxide (1k)<smiles>C/C(=C\C(=P)C1CCCCC1)C(=O)Oc1c(C)cccc1C</smiles>

Pale yellow oil. Yield: $48 \% .{ }^{1} \mathbf{H}$ NMR $\left(400 \mathrm{MHz}, \mathrm{CDCl}_{3}\right) \delta 7.88-7.77(\mathrm{~m}, 4 \mathrm{H}), 7.55-7.41(\mathrm{~m}$, $6 \mathrm{H}), 6.99-6.83(\mathrm{~m}, 3 \mathrm{H}), 4.54-4.41(\mathrm{~m}, 2 \mathrm{H}), 2.14(\mathrm{~s}, 6 \mathrm{H}), 1.74-1.52(\mathrm{~m}, 8 \mathrm{H}), 1.40(\mathrm{~d}, J=13.0$ $\mathrm{Hz}, 1 \mathrm{H}), 1.22-0.86(\mathrm{~m}, 4 \mathrm{H}), 0.64-0.47(\mathrm{~m}, 1 \mathrm{H}) .{ }^{13} \mathbf{C} \mathbf{N M R}\left(101 \mathrm{MHz}, \mathrm{CDCl}_{3}\right) \delta 209.2(\mathrm{~d}, J=$ $6.4 \mathrm{~Hz}), 155.4,133.1(\mathrm{~d}, J=48.5 \mathrm{~Hz}), 132.3,132.2(\mathrm{~d}, J=10.1 \mathrm{~Hz}), 131.8(\mathrm{~d}, J=10.1 \mathrm{~Hz}), 131.7$, 131.2, 128.7, $128.2(\mathrm{~d}, J=13.1 \mathrm{~Hz}), 128.1(\mathrm{~d}, J=12.1 \mathrm{~Hz}), 123.9,108.6(\mathrm{~d}, J=13.3 \mathrm{~Hz}), 96.6$, 95.5, $68.1(\mathrm{~d}, J=12.7 \mathrm{~Hz}), 41.1(\mathrm{~d}, J=4.7 \mathrm{~Hz}), 31.3(\mathrm{~d}, J=2.0 \mathrm{~Hz}), 30.9(\mathrm{~d}, J=2.7 \mathrm{~Hz}), 26.2(\mathrm{~d}$, $J=3.0 \mathrm{~Hz}), 26.1,16.2,16.1(\mathrm{~d}, J=6.1 \mathrm{~Hz}) .{ }^{31} \mathbf{P}$ NMR $\left(162 \mathrm{MHz}, \mathrm{CDCl}_{3}\right) \delta 29.85(\mathrm{~s})$. HRMS (ESI): $\left([\mathrm{M}+\mathrm{Na}]^{+}\right)$Calcd for $\mathrm{C}_{31} \mathrm{H}_{35} \mathrm{NaO}_{2} \mathrm{P}: 493.2272$, Found: 493.2266.

\section{(1-(2,6-dimethylphenoxy)-5-(4-methoxyphenyl)-4-methylpenta-2,3-dien-2-yl)diphenylphos-} phine oxide (1m)<smiles>COc1ccc(C/C(C)=C/C(=P)Pc2ccccc2)cc1</smiles>

A white solid. ${ }^{1} \mathbf{H}$ NMR $\left(400 \mathrm{MHz}, \mathrm{CDCl}_{3}\right) \delta 7.68-7.59(\mathrm{~m}, 2 \mathrm{H}), 7.58-7.48(\mathrm{~m}, 4 \mathrm{H}), 7.47-7.36$ $(\mathrm{m}, 4 \mathrm{H}), 6.95(\mathrm{~d}, J=7.3 \mathrm{~Hz}, 2 \mathrm{H}), 6.88(\mathrm{~d}, J=8.5 \mathrm{~Hz}, 3 \mathrm{H}), 6.77(\mathrm{~d}, J=8.4 \mathrm{~Hz}, 2 \mathrm{H}), 4.48-4.32(\mathrm{~m}$, 2H), $3.81(\mathrm{~s}, 3 \mathrm{H}), 3.20-2.91(\mathrm{~m}, 2 \mathrm{H}), 2.16(\mathrm{~s}, 6 \mathrm{H}), 1.53(\mathrm{~d}, J=5.7 \mathrm{~Hz}, 3 \mathrm{H}) .{ }^{13} \mathbf{C}$ NMR $(101 \mathrm{MHz}$, $\left.\mathrm{CDCl}_{3}\right) \delta 209.1(\mathrm{~d}, J=5.8 \mathrm{~Hz}), 158.3,155.4,132.9,132.0(\mathrm{~d}, J=10.0 \mathrm{~Hz}), 131.9(\mathrm{~d}, J=3.0 \mathrm{~Hz})$, 
$131.8(\mathrm{~d}, J=10.1 \mathrm{~Hz}), 131.7(\mathrm{~d}, J=3.0 \mathrm{~Hz}), 131.1,130.9,130.3,129.8(\mathrm{~d}, J=2.0 \mathrm{~Hz}), 128.7,128.3$ $(\mathrm{d}, J=12.3 \mathrm{~Hz}), 128.1(\mathrm{~d}, J=12.3 \mathrm{~Hz}), 124.0,113.7,104.4(\mathrm{~d}, J=14.1 \mathrm{~Hz}), 96.7,95.6,67.8(\mathrm{~d}, J$ $=13.1 \mathrm{~Hz}), 55.3,38.9(\mathrm{~d}, J=5.2 \mathrm{~Hz}), 17.7(\mathrm{~d}, J=5.1 \mathrm{~Hz}), 16.2 .{ }^{31} \mathbf{P}$ NMR $\left(162 \mathrm{MHz}, \mathrm{CDCl}_{3}\right) \delta$ 30.09 (s). HRMS (ESI): $\left([\mathrm{M}+\mathrm{Na}]^{+}\right)$Calcd for $\mathrm{C}_{33} \mathrm{H}_{33} \mathrm{NaO}_{3} \mathrm{P}: 531.2065$, Found: 531.2059 .

\section{1,3-dimethyl-2-((4-methyl-2-tosylpenta-2,3-dien-1-yl)oxy)benzene $\left(1^{\prime}\right)^{3}$}<smiles>CC(C)=CC(COc1c(C)cccc1C)S(=O)(=O)c1ccc(C)cc1</smiles>

Yellow oil. ${ }^{1} \mathbf{H}$ NMR $\left(400 \mathrm{MHz}, \mathrm{CDCl}_{3}\right) \delta 7.82(\mathrm{~d}, J=8.1 \mathrm{~Hz}, 2 \mathrm{H}), 7.31(\mathrm{~d}, J=8.1 \mathrm{~Hz}, 2 \mathrm{H}), 6.99-$ $6.85(\mathrm{~m}, 3 \mathrm{H}), 4.51(\mathrm{~s}, 2 \mathrm{H}), 2.43(\mathrm{~s}, 3 \mathrm{H}), 2.17(\mathrm{~s}, 6 \mathrm{H}), 1.80(\mathrm{~s}, 6 \mathrm{H}) .{ }^{13} \mathbf{C} \mathbf{N M R}\left(101 \mathrm{MHz}, \mathrm{CDCl}_{3}\right) \delta$ 204.3, 155.4, 144.1, 138.4, 131.0, 129.6, 128.8, 127.9, 124.2, 108.6, 107.3, 67.5, 21.6, 19.5, 16.3. HRMS (ESI): ([M+Na $\left.]^{+}\right)$Calcd for $\mathrm{C}_{21} \mathrm{H}_{24} \mathrm{NaO}_{3} \mathrm{~S}: 379.1344$, Found: 397.1338 .

\section{diethyl (3-methyl-1-phenylbuta-1,2-dien-1-yl)phosphonate (1") $)^{7}$}

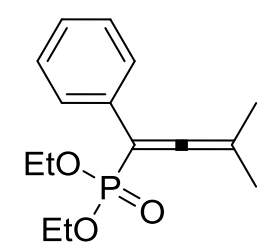

Pale yellow oil. Yield: 50\%. ${ }^{1} \mathbf{H}$ NMR (400 MHz, $\left.\mathrm{CDCl}_{3}\right) \delta 7.54(\mathrm{~d}, J=7.9 \mathrm{~Hz}, 2 \mathrm{H}), 7.36-7.20$ (m, 3H), $4.18-4.06(\mathrm{~m}, 4 \mathrm{H}), 1.89$ (d, $J=6.4 \mathrm{~Hz}, 6 \mathrm{H}), 1.30$ (t, $J=7.1 \mathrm{~Hz}, 6 \mathrm{H}) .{ }^{13} \mathbf{C} \mathbf{N M R}\left(101 \mathrm{MHz}, \mathrm{CDCl}_{3}\right)$ $\delta 209.2(\mathrm{~d}, J=4.2 \mathrm{~Hz}), 133.2(\mathrm{~d}, J=10.4 \mathrm{~Hz}), 128.5,127.7$ (d, $J=5.9 \mathrm{~Hz}), 127.3,99.3(\mathrm{~d}, J=15.4 \mathrm{~Hz})$, 96.1, 94.2, $62.5(\mathrm{~d}, J=6.1 \mathrm{~Hz}), 61.8,19.5(\mathrm{~d}, J=6.6 \mathrm{~Hz}), 16.3(\mathrm{~d}, J=6.6 \mathrm{~Hz}) .{ }^{31} \mathbf{P}$ NMR $(162 \mathrm{MHz}$, $\left.\mathrm{CDCl}_{3}\right) \delta 16.53(\mathrm{~s})$.

\section{References}

1. Mao, M.; Zhang, L.; Chen, Y.-Z.; Zhu, J.; Wu, L. ACS Catal. 2017, 7, 181-185.

2. Chen, Y.-Z.; Zhang, L.; Lu, A.-M.; Yang, F.; Wu, L. J. Org. Chem. 2015, 80, 673-680.

3. Liu, T.; Sun, X.; Wu, L. Adv. Synth. Catal. 2018, 360, 2005-2012.

4. Zhang, L.; Zhu, J.; Ma, J.; Wu, L.; Zhang, W.-H. Org. Lett. 2017, 19, 6308-6311. 
5. Luo, K.; Zhang, L.; Ma, J.; Sha, Q.; Wu, L. J. Org. Chem. 2017, 82, 6978-6985.

6. Mao, L.-L.; Li, Y.-H.; Yang, S.-D. Org. Chem. Front., 2017, 4, 608-611.

7. (a) Yu, F.; Lian, X.; Zhao, J.; Yu, Y.; Ma, S. J. Org. Chem. 2009, 74, 1130; (b) Xin, N.; Ma, S. Eur. J. Org. Chem. 2012, 3806.

For Characterizations of New Compounds: 11, 1n, 1s, 1v, 1ac, 1ad, 1af, 1ag, 1aj. (1-(2,6-dimethylphenoxy)-4,5,5-trimethylhexa-2,3-dien-2-yl)diphenylphosphine oxide (11)<smiles>CC(=CC(COc1c(C)cccc1C)PP)C(C)(C)C</smiles>

A white solid, yield: $46 \%$, m.p.: $97.6-98.7^{\circ} \mathrm{C} .{ }^{1} \mathbf{H}$ NMR $\left(400 \mathrm{MHz}, \mathrm{CDCl}_{3}\right) \delta 7.98-7.71(\mathrm{~m}, 4 \mathrm{H})$, $7.57-7.36(\mathrm{~m}, 6 \mathrm{H}), 7.00-6.80(\mathrm{~m}, 3 \mathrm{H}), 4.60-4.30(\mathrm{~m}, 2 \mathrm{H}), 2.14$ (s, 6H), $1.62(\mathrm{~d}, J=5.8 \mathrm{~Hz}$, 3H), $0.81(\mathrm{~s}, 9 \mathrm{H}) .{ }^{13} \mathbf{C}$ NMR $\left(101 \mathrm{MHz}, \mathrm{CDCl}_{3}\right) \delta 208.8(\mathrm{~d}, J=6.9 \mathrm{~Hz}), 155.3,133.4,132.5(\mathrm{~d}, J=$ $22.2 \mathrm{~Hz}), 132.1(\mathrm{~d}, J=9.7 \mathrm{~Hz}), 131.8,131.6(\mathrm{~d}, J=26.3 \mathrm{~Hz}), 131.4$ (d, $J=56.6 \mathrm{~Hz}), 128.7,128.3$ $(\mathrm{d}, J=10.1 \mathrm{~Hz}), 128.2(\mathrm{~d}, J=10.1 \mathrm{~Hz}), 124.0,112.2(\mathrm{~d}, J=13.4 \mathrm{~Hz}), 96.3,95.2,67.9$ (d, $J=12.6$ $\mathrm{Hz}), 33.6(\mathrm{~d}, J=4.3 \mathrm{~Hz}), 28.4,16.1,14.0(\mathrm{~d}, J=5.6 \mathrm{~Hz}) .{ }^{31} \mathbf{P}$ NMR $\left(162 \mathrm{MHz}, \mathrm{CDCl}_{3}\right) \delta 30.35(\mathrm{~s})$. HRMS (ESI): ([M+Na] $]^{+}$) Calcd for $\mathrm{C}_{29} \mathrm{H}_{33} \mathrm{NaO}_{2} \mathrm{P}: 467.2116$, Found: 467.2110.

(4-benzyl-1-(2,6-dimethylphenoxy)-5-phenylpenta-2,3-dien-2-yl)diphenylphosphine oxide (1n)<smiles>Cc1cccc(C)c1OCC(=C=C(Br)Cc1ccccc1)P=O</smiles>

Pale yellow oil, yield: $53 \%{ }^{1}{ }^{1} \mathbf{H}$ NMR $\left(400 \mathrm{MHz}, \mathrm{CDCl}_{3}\right) \delta 7.54-7.43(\mathrm{~m}, 6 \mathrm{H}), 7.42-7.36(\mathrm{~m}$, 4H), $7.27-7.20(\mathrm{~m}, 6 \mathrm{H}), 7.02-6.92(\mathrm{~m}, 6 \mathrm{H}), 6.92-6.85(\mathrm{~m}, 1 \mathrm{H}), 4.36(\mathrm{~d}, J=7.8 \mathrm{~Hz}, 2 \mathrm{H}), 3.15$ $(\mathrm{qd}, J=15.4,5.5 \mathrm{~Hz}, 4 \mathrm{H}), 2.16(\mathrm{~s}, 6 \mathrm{H}) .{ }^{13} \mathbf{C}$ NMR $\left(101 \mathrm{MHz}, \mathrm{CDCl}_{3}\right) \delta 208.5(\mathrm{~d}, J=6.1 \mathrm{~Hz}), 155.3$, $137.7(\mathrm{~d}, J=2.3 \mathrm{~Hz}), 132.3,131.9,131.8,131.8$ (d, $J=12.1 \mathrm{~Hz}), 131.2,131.2,129.3,128.8,128.4$, 128.3, 128.2, 126.6, 124.1, $108.3(\mathrm{~d}, J=13.3 \mathrm{~Hz}), 98.1,97.0,67.6(\mathrm{~d}, J=13.4 \mathrm{~Hz}), 38.3(\mathrm{~d}, J=5.0$ Hz), 16.2. ${ }^{31}$ P NMR $\left(162 \mathrm{MHz}, \mathrm{CDCl}_{3}\right) \delta 28.76$ (s). HRMS (ESI): ([M+H] $\left.]^{+}\right)$Calcd for $\mathrm{C}_{38} \mathrm{H}_{36} \mathrm{O}_{2} \mathrm{P}$ : 555.2453, Found: 555.2447.

(4-cyclohexyl-1-(2,6-dimethylphenoxy)buta-2,3-dien-2-yl)diphenylphosphine oxide (1s) 
<smiles>Cc1cccc(C)c1OCC(=CC1CCCCC1)P=O</smiles>

Colorless oil, yield: 36\%. ${ }^{1} \mathbf{H}$ NMR $\left(400 \mathrm{MHz}, \mathrm{CDCl}_{3}\right) \delta 7.86-7.78(\mathrm{~m}, 4 \mathrm{H}), 7.46-7.40(\mathrm{~m}, 6 \mathrm{H})$, $6.89(\mathrm{~d}, J=7.3 \mathrm{~Hz}, 2 \mathrm{H}), 6.83-6.80(\mathrm{~m}, 1 \mathrm{H}), 5.32-5.28(\mathrm{~m}, 1 \mathrm{H}), 4.49(\mathrm{~d}, J=9.4 \mathrm{~Hz}, 2 \mathrm{H}), 2.13(\mathrm{~s}$, 6H), $1.84(\mathrm{~s}, 1 \mathrm{H}), 1.59-1.42(\mathrm{~m}, 5 \mathrm{H}), 1.17-1.08(\mathrm{~m}, 2 \mathrm{H}), 1.03-0.97(\mathrm{~m}, 1 \mathrm{H}), 0.84-0.68(\mathrm{~m}$, 2H). ${ }^{13} \mathrm{C} \mathrm{NMR}\left(101 \mathrm{MHz}, \mathrm{CDCl}_{3}\right) \delta 209.8(\mathrm{~d}, J=6.4 \mathrm{~Hz}), 155.2,132.7(\mathrm{~d}, J=13.2 \mathrm{~Hz}), 132.0(\mathrm{~d}$, $J=9.6 \mathrm{~Hz}), 131.9,131.8,131.6(\mathrm{~d}, J=14.4 \mathrm{~Hz}), 131.0,128.7,128.2(\mathrm{~d}, J=2.3 \mathrm{~Hz}), 128.2(\mathrm{~d}, J=$ $22.0 \mathrm{~Hz}), 124.1,100.3$ (d, $J=12.7 \mathrm{~Hz}), 97.2(\mathrm{~d}, J=104.6 \mathrm{~Hz}), 67.9$ (d, $J=11.2 \mathrm{~Hz}), 36.7$ (d, $J=$ $4.6 \mathrm{~Hz}), 32.6(\mathrm{~d}, J=2.6 \mathrm{~Hz}), 32.2,25.8,25.8(\mathrm{~d}, J=3.6 \mathrm{~Hz}), 16.2 .{ }^{31} \mathbf{P} \mathbf{N M R}\left(162 \mathrm{MHz}, \mathrm{CDCl}_{3}\right) \delta$ 28.5 (s). HRMS (ESI): ([M+Na $\left.]^{+}\right)$Calcd for $\mathrm{C}_{30} \mathrm{H}_{33} \mathrm{O}_{2} \mathrm{NaP}: 479.2116$, Found: 479.2110.

(1-(2,6-dimethylphenoxy)-4-methylpenta-2,3-dien-2-yl)di(thiophen-2-yl)phosphine oxide (1v)

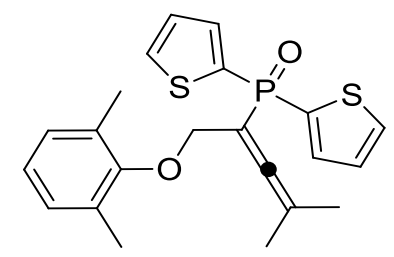

Pale yellow oil, yield: $65 \%$. ${ }^{1} \mathrm{H}$ NMR $\left(400 \mathrm{MHz}, \mathrm{CDCl}_{3}\right) \delta 7.73(\mathrm{t}, J=4.5 \mathrm{~Hz}, 2 \mathrm{H}), 7.65(\mathrm{dd}, J=$ 7.6, 3.6 Hz, 2H), $7.21-7.15(\mathrm{~m}, 2 \mathrm{H}), 7.02-6.82(\mathrm{~m}, 3 \mathrm{H}), 4.55(\mathrm{~d}, J=9.6 \mathrm{~Hz}, 2 \mathrm{H}), 2.21(\mathrm{~s}, 6 \mathrm{H})$, $1.64(\mathrm{~d}, J=6.5 \mathrm{~Hz}, 6 \mathrm{H}) .{ }^{13} \mathbf{C}$ NMR $\left(101 \mathrm{MHz}, \mathrm{CDCl}_{3}\right) \delta 208.6(\mathrm{~d}, J=5.8 \mathrm{~Hz}), 155.6,136.4(\mathrm{~d}, J=$ $10.4 \mathrm{~Hz}), 134.9,133.7$ (d, $J=5.2 \mathrm{~Hz}), 131.2,128.7,128.1$ (d, $J=14.6 \mathrm{~Hz}), 124.0,100.9$ (d, $J=$ 14.5 Hz), 96.4 (d, $J=118.2 \mathrm{~Hz}), 67.9$ (d, $J=14.1 \mathrm{~Hz}), 19.2$ (d, $J=6.1 \mathrm{~Hz}), 16.3 .{ }^{31} \mathbf{P}$ NMR $(162$ $\left.\mathrm{MHz}, \mathrm{CDCl}_{3}\right) \delta 14.95$ (s). HRMS (ESI): ([M+Na $\left.]^{+}\right)$Calcd for $\mathrm{C}_{22} \mathrm{H}_{23} \mathrm{NaO}_{2} \mathrm{PS}_{2}: 437.0775$, Found: 437.0769 .

(2-cyclobutylidene-1-phenylvinyl)diphenylphosphine oxide (1ac)<smiles>O=PP(c1ccccc1)c1ccccc1</smiles>

A white solid, yield: 82\%, m.p.: 91.9-92.7 ${ }^{\circ} \mathrm{C} .{ }^{1} \mathbf{H}$ NMR (400 MHz, $\left.\mathrm{CDCl}_{3}\right) \delta 7.82-7.70(\mathrm{~m}, 4 \mathrm{H})$, $7.61(\mathrm{~d}, J=8.0 \mathrm{~Hz}, 2 \mathrm{H}), 7.55-7.48(\mathrm{~m}, 2 \mathrm{H}), 7.48-7.40(\mathrm{~m}, 4 \mathrm{H}), 7.26-7.13(\mathrm{~m}, 3 \mathrm{H}), 2.96-2.80$ $(\mathrm{m}, 2 \mathrm{H}), 2.43-2.27(\mathrm{~m}, 2 \mathrm{H}), 1.87-1.71(\mathrm{~m}, 1 \mathrm{H}), 1.57-1.40(\mathrm{~m}, 1 \mathrm{H}) .{ }^{13} \mathbf{C} \mathbf{N M R}\left(101 \mathrm{MHz}, \mathrm{CDCl}_{3}\right)$ 
$\delta 203.1(\mathrm{~d}, J=6.1 \mathrm{~Hz}), 133.6,133.4,133.4,132.5,131.7,131.6,131.6,128.5,128.3,128.3,128.2$, 127.4, $105.5(\mathrm{~d}, J=5.0 \mathrm{~Hz}), 103.3(\mathrm{~d}, J=2.0 \mathrm{~Hz}), 28.9(\mathrm{~d}, J=6.1 \mathrm{~Hz}), 17.5 .{ }^{31} \mathbf{P}$ NMR $(162 \mathrm{MHz}$, $\left.\mathrm{CDCl}_{3}\right) \delta 31.59$ (s). HRMS (ESI): $\left([\mathrm{M}+\mathrm{H}]^{+}\right)$Calcd for $\mathrm{C}_{24} \mathrm{H}_{22} \mathrm{OP}: 357.1408$, Found: 357.1402.

\section{(2-cyclopropylidene-1-phenylvinyl)diphenylphosphine oxide (1ad)}<smiles>O=P[PH](c1ccccc1)(c1ccccc1)C1CC1</smiles>

A white solid, yield: $76 \%$, m.p.: $152.8-153.7^{\circ} \mathrm{C} .{ }^{1} \mathbf{H}$ NMR $\left(400 \mathrm{MHz}, \mathrm{CDCl}_{3}\right) \delta 7.82-7.71(\mathrm{~m}$, 4H), $7.62(\mathrm{~d}, J=7.7 \mathrm{~Hz}, 2 \mathrm{H}), 7.48-7.34(\mathrm{~m}, 6 \mathrm{H}), 7.26-7.08(\mathrm{~m}, 3 \mathrm{H}), 1.65(\mathrm{q}, J=6.7 \mathrm{~Hz}, 2 \mathrm{H})$, $1.12(\mathrm{q}, J=6.6 \mathrm{~Hz}, 2 \mathrm{H}) .{ }^{13} \mathbf{C}$ NMR $\left(101 \mathrm{MHz}, \mathrm{CDCl}_{3}\right) \delta 195.9$ (d, $\left.J=7.6 \mathrm{~Hz}\right), 133.4$ (d, $J=7.1$ $\mathrm{Hz}), 133.4,132.3,131.7,131.6,131.6,128.5,128.2$ (d, $J=5.1 \mathrm{~Hz}), 128.0$ (d, $J=5.1 \mathrm{~Hz}), 127.1$, 102.3, 101.3, 79.6, 79.5, $10.8(\mathrm{~d}, J=4.3 \mathrm{~Hz}) .{ }^{31} \mathbf{P}$ NMR $\left(162 \mathrm{MHz}, \mathrm{CDCl}_{3}\right) \delta 31.59$ (s). HRMS (ESI): $\left([\mathrm{M}+\mathrm{H}]^{+}\right.$) Calcd for $\mathrm{C}_{23} \mathrm{H}_{20} \mathrm{OP}: 343.1252$, Found: 343.1246 .

\section{(3-methyl-1-phenylhepta-1,2,6-trien-1-yl)diphenylphosphine oxide (1af)}<smiles>C=CCC/C(C)=C/C(=PP)c1ccccc1</smiles>

A white solid, yield: $62 \%$, m.p.: $63.9-64.1{ }^{\circ} \mathrm{C} .{ }^{1} \mathbf{H}$ NMR $\left(400 \mathrm{MHz}, \mathrm{CDCl}_{3}\right) \delta 7.80-7.69$ (m, 4H), $7.62(\mathrm{~d}, J=7.8 \mathrm{~Hz}, 2 \mathrm{H}), 7.49-7.37(\mathrm{~m}, 6 \mathrm{H}), 7.27-7.09$ (m, 3H), $5.82-5.45(\mathrm{~m}, 1 \mathrm{H}), 4.93$ (t, $J=$ $12.4 \mathrm{~Hz}, 2 \mathrm{H}), 1.96-1.86(\mathrm{~m}, 2 \mathrm{H}), 1.85-1.67(\mathrm{~m}, 2 \mathrm{H}), 1.53(\mathrm{~d}, J=5.9 \mathrm{~Hz}, 3 \mathrm{H}) .{ }^{13} \mathbf{C}$ NMR $(101$ $\left.\mathrm{MHz}, \mathrm{CDCl}_{3}\right) \delta 209.5(\mathrm{~d}, J=6.1 \mathrm{~Hz}), 137.4,133.5(\mathrm{~d}, J=25.3 \mathrm{~Hz}), 133.3,132.4(\mathrm{~d}, J=33.3 \mathrm{~Hz})$, 131.7, $131.6(\mathrm{~d}, J=9.1 \mathrm{~Hz}), 131.5(\mathrm{~d}, J=14.1 \mathrm{~Hz}), 128.3(\mathrm{~d}, J=37.4 \mathrm{~Hz}), 128.3,128.2(\mathrm{~d}, J=29.3$ Hz), 127.3, 115.2, 104.1, 104.0, 101.0, 100.0, 32.7 (d, $J=5.2 \mathrm{~Hz}), 31.3$ (d, $J=2.0 \mathrm{~Hz}), 17.7$ (d, $J=$ $6.1 \mathrm{~Hz}) .{ }^{31} \mathbf{P}$ NMR $\left(162 \mathrm{MHz}, \mathrm{CDCl}_{3}\right) \delta 31.44$ (s). HRMS (ESI): $\left([\mathrm{M}+\mathrm{H}]^{+}\right)$Calcd for $\mathrm{C}_{26} \mathrm{H}_{26} \mathrm{OP}: 385.1721$, Found: 385.1715 .

\section{(1-(4-bromophenyl)-3-methylbuta-1,2-dien-1-yl)diphenylphosphine oxide (1ag)}<smiles>CC(C)=C=C(P=P)c1ccc(Br)cc1</smiles> 
A white solid, yield: $72 \%$, m.p.: $108.1-109.0{ }^{\circ} \mathrm{C} .{ }^{1} \mathbf{H}$ NMR $\left(400 \mathrm{MHz}, \mathrm{CDCl}_{3}\right) \delta 7.79-7.70(\mathrm{~m}$, 4H), $7.58(\mathrm{~d}, J=7.4 \mathrm{~Hz}, 2 \mathrm{H}), 7.53-7.39(\mathrm{~m}, 6 \mathrm{H}), 7.27-7.13(\mathrm{~m}, 2 \mathrm{H}), 1.47$ (d, $J=6.0 \mathrm{~Hz}, 6 \mathrm{H})$. ${ }^{13} \mathbf{C}$ NMR $\left(101 \mathrm{MHz}, \mathrm{CDCl}_{3}\right) \delta 210.1,133.5,132.4,131.6,131.6,131.6,131.5,128.5,128.3,128.3$, 128.2, 128.2, 127.3, 119.7, $100.0(\mathrm{~d}, J=13.7 \mathrm{~Hz}), 19.0(\mathrm{~d}, J=5.8 \mathrm{~Hz}) .{ }^{31} \mathbf{P} \mathbf{N M R}\left(162 \mathrm{MHz}, \mathrm{CDCl}_{3}\right)$ $\delta 31.66$ (s). HRMS (ESI): $\left([\mathrm{M}+\mathrm{H}]^{+}\right)$Calcd for $\mathrm{C}_{23} \mathrm{H}_{21} \mathrm{OBrP}: 423.0513$, Found: 423.0507.

(4,4-dicyclopropyl-1-(2,6-dimethylphenoxy)buta-2,3-dien-2-yl)diphenylphosphine oxide (1aj)<smiles>Cc1cccc(C)c1OCC(C=C(C1CC1)C1CC1)P=O</smiles>

Pale yellow oil, yield: 38\%. ${ }^{1} \mathbf{H}$ NMR (400 MHz, $\left.\mathrm{CDCl}_{3}\right) \delta 7.88-7.77(\mathrm{~m}, 4 \mathrm{H}), 7.55-7.42$ (m, $6 \mathrm{H}), 6.96-6.82(\mathrm{~m}, 3 \mathrm{H}), 4.43(\mathrm{~d}, J=8.8 \mathrm{~Hz}, 2 \mathrm{H}), 2.12(\mathrm{~s}, 6 \mathrm{H}), 1.17-1.03(\mathrm{~m}, 2 \mathrm{H}), 0.68-0.50$ (m, 4H), $0.36-0.24(\mathrm{~m}, 2 \mathrm{H}), 0.21-0.07(\mathrm{~m}, 2 \mathrm{H}) .{ }^{13} \mathbf{C}$ NMR $\left(101 \mathrm{MHz}, \mathrm{CDCl}_{3}\right) \delta 209.3(\mathrm{~d}, J=6.2$ Hz), 155.3, 132.8, 131.9, 131.8, 131.8, 131.7, 131.1, 128.7, 128.2 (d, $J=12.2 \mathrm{~Hz}), 124.0,114.4$ (d, $J=13.0 \mathrm{~Hz}), 99.5,98.5,67.8(\mathrm{~d}, J=13.2 \mathrm{~Hz}), 16.1,11.4(\mathrm{~d}, J=5.9 \mathrm{~Hz}), 5.9(\mathrm{~d}, J=2.3 \mathrm{~Hz}), 5.5(\mathrm{~d}$, $J=2.1 \mathrm{~Hz}) .{ }^{31} \mathbf{P}$ NMR $\left(162 \mathrm{MHz}, \mathrm{CDCl}_{3}\right) \delta 28.37$ (s). HRMS (ESI): $\left([\mathrm{M}+\mathrm{H}]^{+}\right)$Calcd for $\mathrm{C}_{30} \mathrm{H}_{32} \mathrm{O}_{2} \mathrm{P}$ : 455.2140, Found: 455.2134 . 


\section{General Procedures for Visible-Light-Driven C-P Cleavage and Migration of Allenylphosphine Oxides}

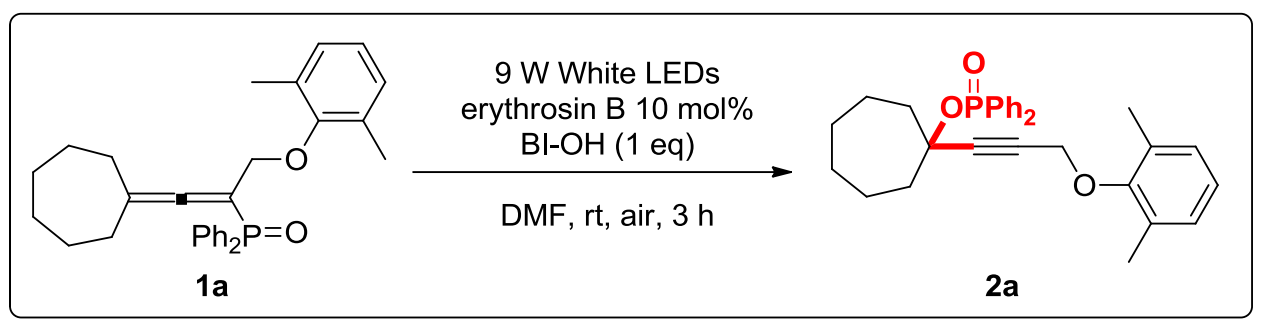

To a $10 \mathrm{~mL}$ tube was added allenylphosphine oxide (1a, $0.2 \mathrm{mmol})$, erythrosin B (16.7 mg, 10 mol\%), 1-hydroxy-2-oxa-1-ioda(III)indan-3-one (52 mg, $0.2 \mathrm{mmol})$ and $2 \mathrm{~mL}$ DMF. The reaction mixture was then stirred for 3 hours irradiated by $9 \mathrm{~W}$ White LEDs at room temperature. The resulting mixture was diluted with ethyl acetate, and then washed with saturated $\mathrm{NaHCO}_{3}$ solution and brine. The organic layer was collected and concentrated. The residue was purified by column chromatography (eluent: 1:2 (v/v) of ethyl acetate/petroleum ether) to afford product $\mathbf{2 a}$ as a white solid.

\section{Gram-Scale Synthesis:}

To a $100 \mathrm{~mL}$ round-bottomed flask was added allenylphosphine oxide (1a, $2 \mathrm{mmol})$, erythrosin B (167 mg, 10 mol\%), 1-hydroxy-2-oxa-1-ioda(III)indan-3-one (520 mg, 2 mmol) and 25 mL DMF. The reaction mixture was then stirred for 5 hours irradiated by $9 \mathrm{~W}$ White LEDs at room temperature. The resulting mixture was diluted with ethyl acetate, and then washed with saturated $\mathrm{NaHCO}_{3}$ solution and brine. The organic layer was collected and concentrated. The residue was purified by column chromatography (eluent: 1:2 (v/v) of ethyl acetate/petroleum ether) to afford product 2a as a white solid ( $830 \mathrm{mg}, 87.8 \%$ yield). 


\section{Optimization of the Reaction Conditions and The Reactions of Single Electron Oxidants}

Table S1. Screening of Photocatalysts a

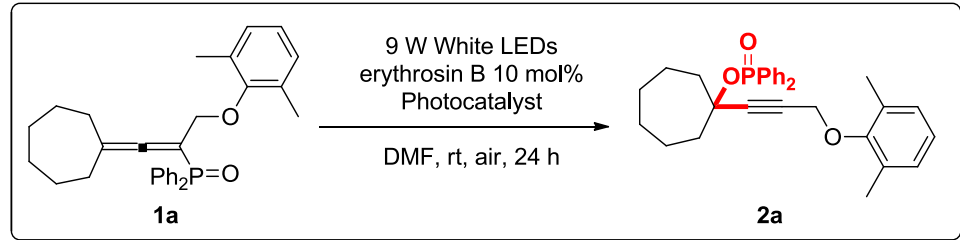

\begin{tabular}{ccc}
\hline entry & photocatalyst & yield (\%) \\
\hline 1 & eosin Y & 30 \\
2 & acid Red 51 & 17 \\
5 & CBr $_{4}$ & $<5$ \\
6 & CuI $_{2}$ & $<5$ \\
7 & phthalocyanine & N.R \\
8 & erythrosin B sodium salt & 22 \\
9 & fluorescein & N.R. \\
10 & pigment Green 7 & N.R. \\
11 & 2,4,6-Triphenylpyrylium Tetrafluoroborate & N.R. \\
12 & p-Anisaldehyde & $<5$ \\
13 & erythrosin B & $>95(81)$ \\
14 & erythrosin B & 50 \\
15 & erythrosin B & 64
\end{tabular}

${ }^{\text {a }}$ Reaction conditions: allenylphosphine oxide (1a, $\left.0.2 \mathrm{mmol}\right)$, photocatalyst (10 mol\%), $2 \mathrm{~mL} \mathrm{DMF,}$ $24 \mathrm{~h}, 9 \mathrm{~W}$ White LEDs, r.t.; ${ }^{\mathrm{b}}$ photocatalyst $(2 \mathrm{~mol} \%)$; ${ }^{\mathrm{c}}$ photocatalyst $(5 \mathrm{~mol} \%)$; ${ }^{\mathrm{d}}$ photocatalyst $(20 \mathrm{~mol} \%)$; ${ }^{\mathrm{e}}$ Yields based on ${ }^{31} \mathrm{P}$ NMR, isolated yields in brackets, N. R. = No Reaction.

Table S2. Screening of Solvents a

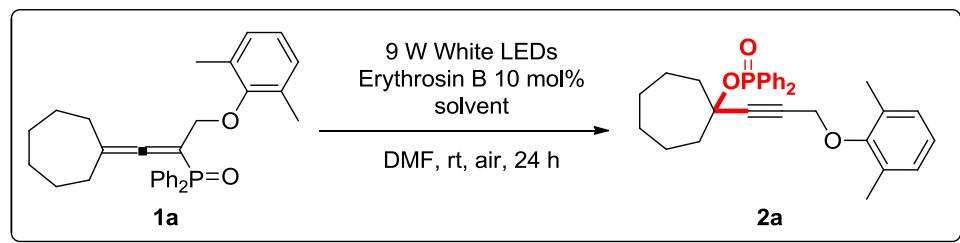

\begin{tabular}{ccc}
\hline entry & solvent & yield $(\%)^{\mathrm{b}}$ \\
\hline 1 & $\mathrm{MeOH}$ & 7 \\
2 & $1,4-\mathrm{Dioxane}$ & N.R. \\
3 & $\mathrm{CH}_{3} \mathrm{NO}_{2}$ & 15
\end{tabular}




$\begin{array}{ccc}4 & \mathrm{CH}_{3} \mathrm{CN} & 18 \\ 5 & \text { DMSO } & 12 \\ 6 & \text { DMAc } & 73(52) \\ 7 & \text { DMF } & >95(81) \\ 8 & \text { Toluene } & \text { Trace } \\ 9 & \text { DCE } & \text { N.R. } \\ 10 & \text { EA } & \text { Trace }\end{array}$

${ }^{a}$ Reaction conditions: allenylphosphine oxide (1a, $\left.0.2 \mathrm{mmol}\right)$, erythrosin B (10 mol\%), $2 \mathrm{~mL}$ solvent, $24 \mathrm{~h}, 9 \mathrm{~W}$ White LEDs, r.t.; ${ }^{\text {}}$ Yields based on ${ }^{31} \mathrm{P}$ NMR, isolated yields in brackets, N. R. $=$ No Reaction.

Table S3. Screening of Additives, Atmosphere and Reaction Time ${ }^{\mathrm{a}}$

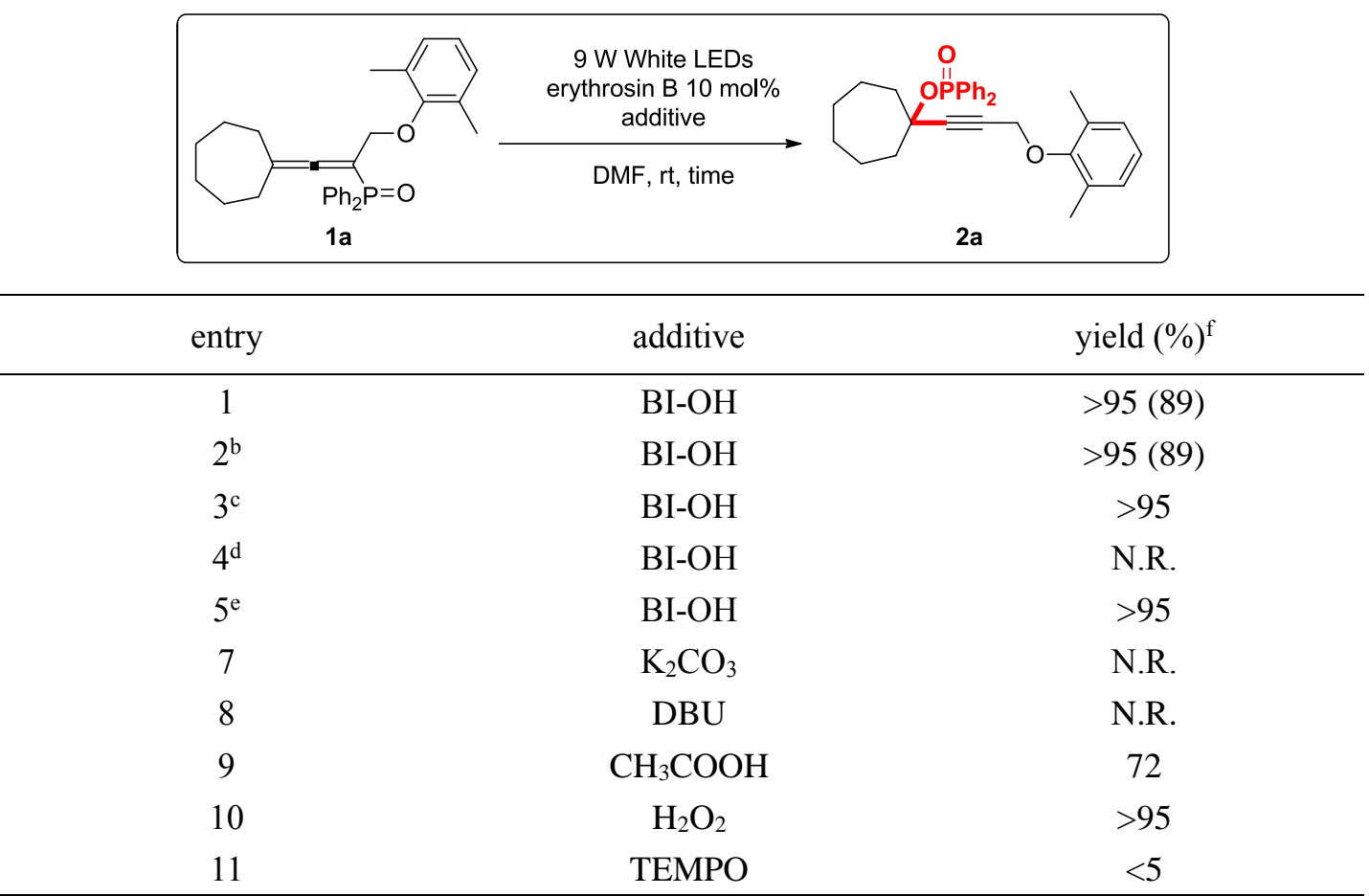

${ }^{a}$ Reaction conditions: allenylphosphine oxide (1a, $\left.0.2 \mathrm{mmol}\right)$, erythrosin B (10 mol\%), additive (1 eq), 2 mL DMF, 24 h, $9 \mathrm{~W}$ White LEDs, r.t.; 3 hours; ${ }^{\mathrm{b}} 6$ hours; ${ }^{\mathrm{d}}$ reaction under $\mathrm{N}_{2}$ atmosphere; ${ }^{\mathrm{e}}$ reaction under $\mathrm{O}_{2}$ atmosphere; ${ }^{\mathrm{f}}$ Yields based on ${ }^{31} \mathrm{P}$ NMR, isolated yields in brackets, N. R. $=$ No Reaction.

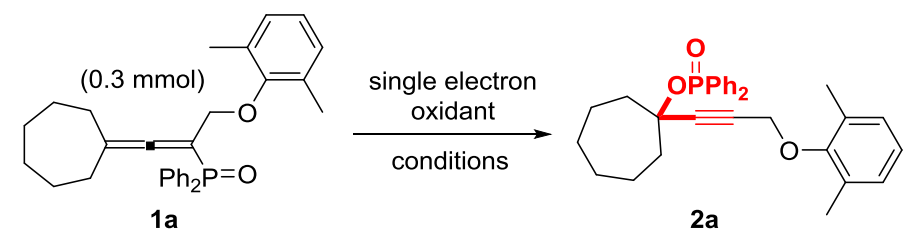

(1) 2 equiv Ceric Ammonium Nitrate, $\mathrm{O}_{2}$ balloon, $\mathrm{CH}_{3} \mathrm{CN}(2 \mathrm{~mL}), 80^{\circ} \mathrm{C}$;

(2) 2 equiv $\mathrm{Mn}(\mathrm{OAc})_{3}-2 \mathrm{H}_{2} \mathrm{O}, \mathrm{O}_{2}$ balloon, toluene $(2 \mathrm{~mL}), 80^{\circ} \mathrm{C}$;

All results of the single electron oxidants were negative:

For condition 1), 1a decomposed rapidly, but without detection of $\mathbf{2 a}$;

For condition 2), $95 \%$ of 1 a was recovered. 


\section{HR-MS Results on the Reaction of $1 p$}

Reaction conditions: 1p (0.2 mmol), erythrosin B (10 mol\%), BI-OH (1 eq), 2 mL DMF, $9 \mathrm{~W}$ White LEDs, r.t. for 3 hours.
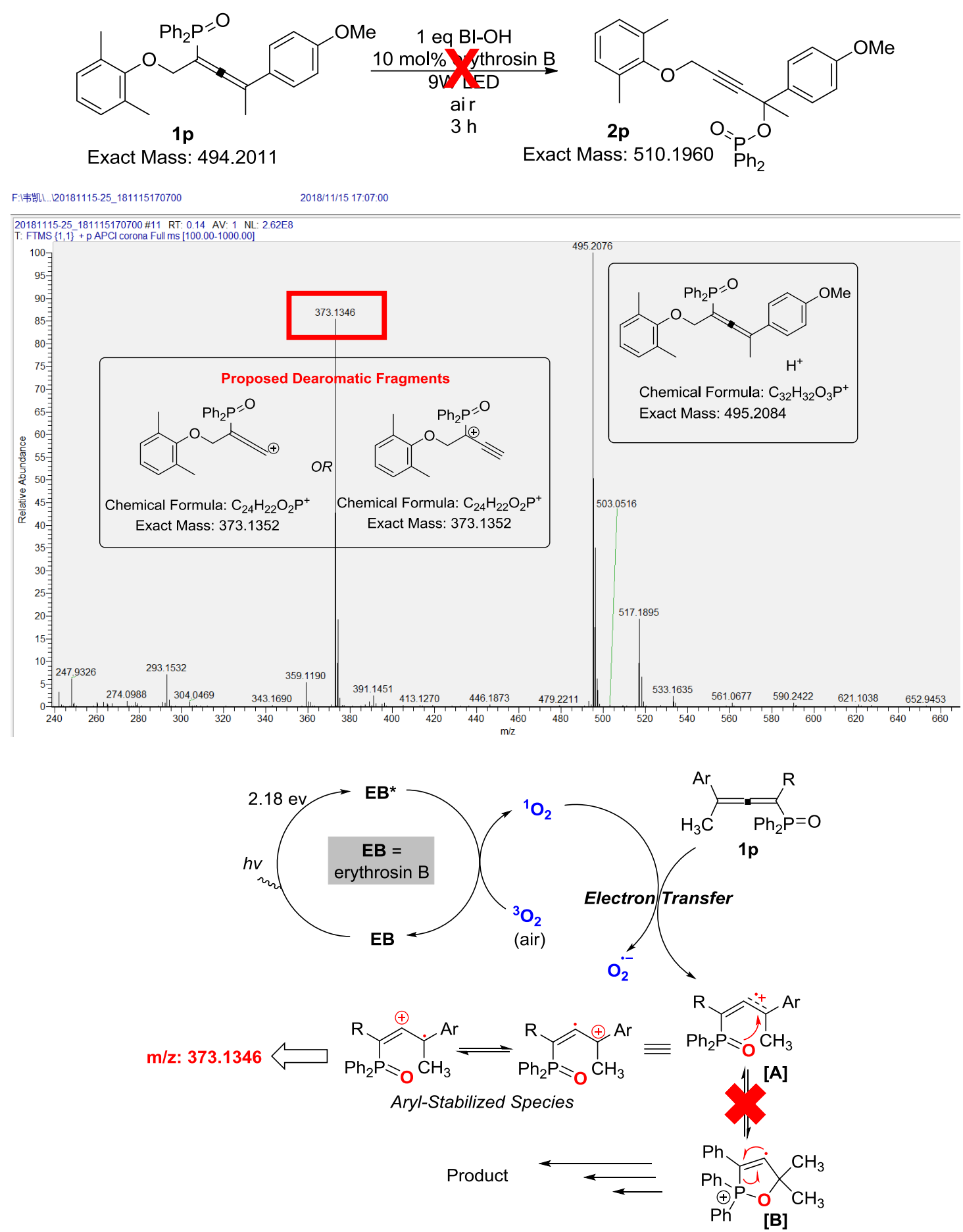

The failure of $2 \mathbf{o}$ and $\mathbf{2 p}$ might be attributed to the stabilization of aryl groups to the intermediate [A], thus altered the pathway to final product. Due to complexity of reaction system, the side products were not identified yet. 


\section{Crossover Experiment}
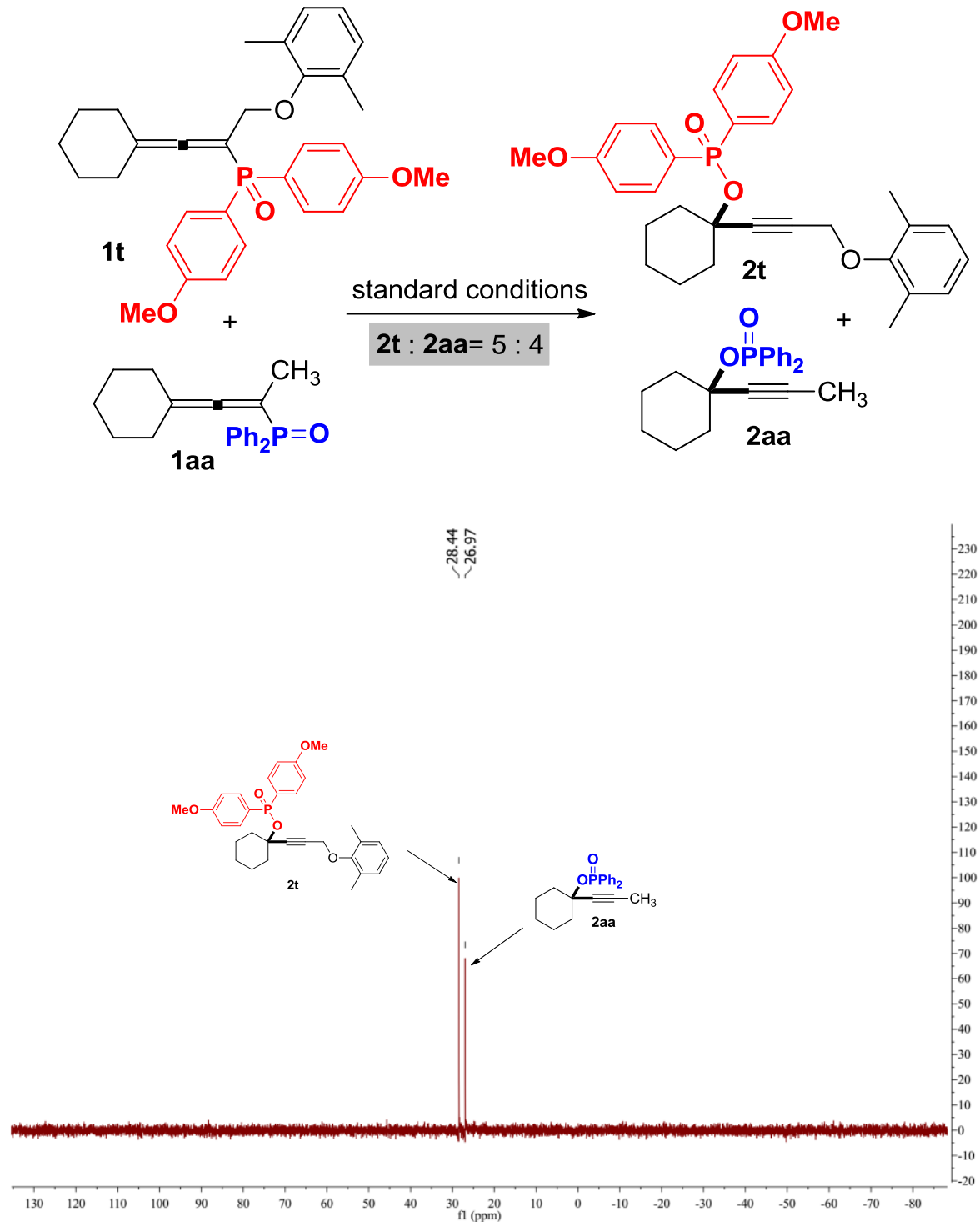

Figure S1. ${ }^{31} \mathrm{P}-\mathrm{NMR}$ analysis of crossover experiments

\section{7. ${ }^{18} \mathrm{O}$-labeling Experiment}

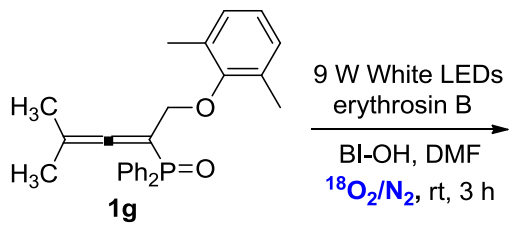

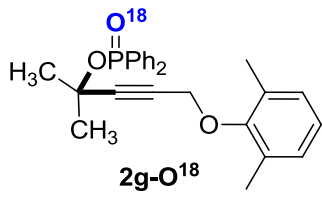

Determined by basic hydrolysis and HR-MS 
NJNY-20180326-2 \#25 RT: 0.37 AV: 1 NL: 8.50E6

T: FTMS $\{1,1\}$ + p ESI Full ms [100.00-1000.00]

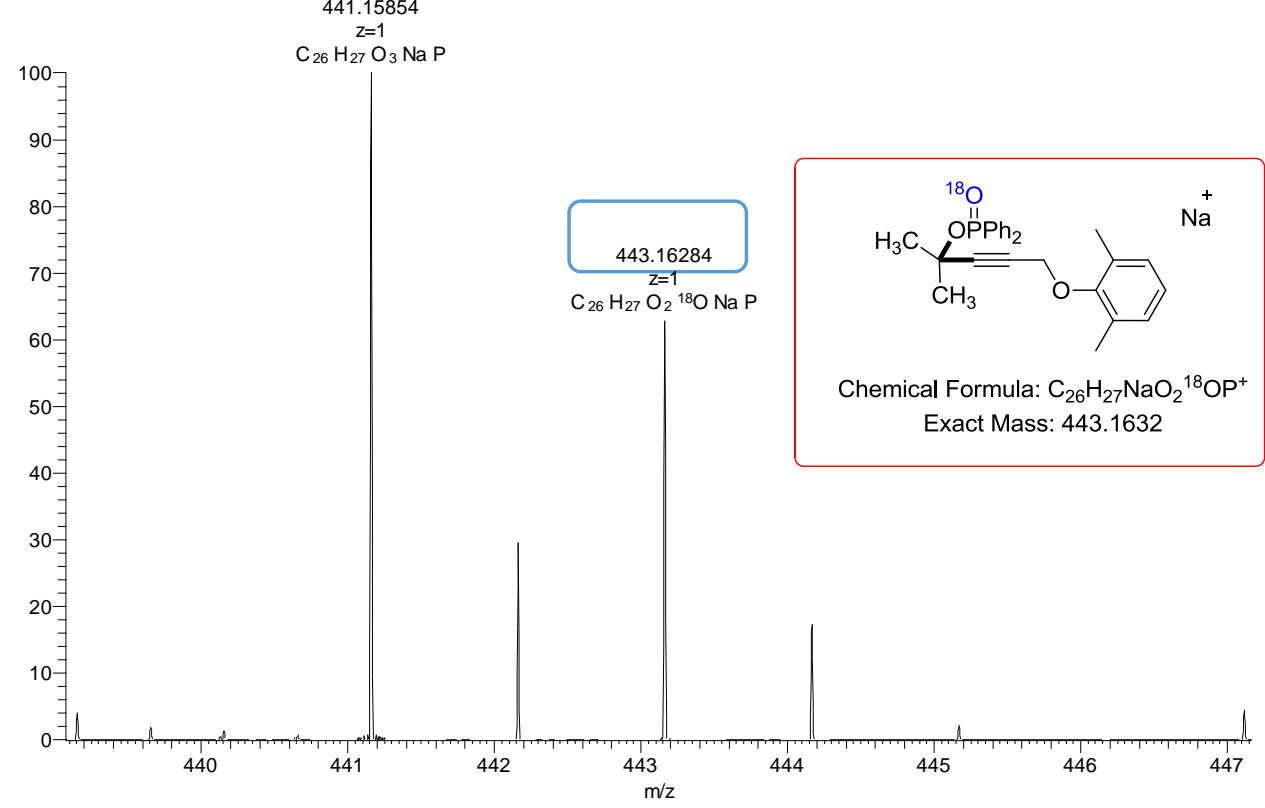

Figure S2. HR-MS result of ${ }^{18} \mathrm{O}$-labeling product $\mathbf{2 g - \mathbf { O } ^ { 1 8 }}$
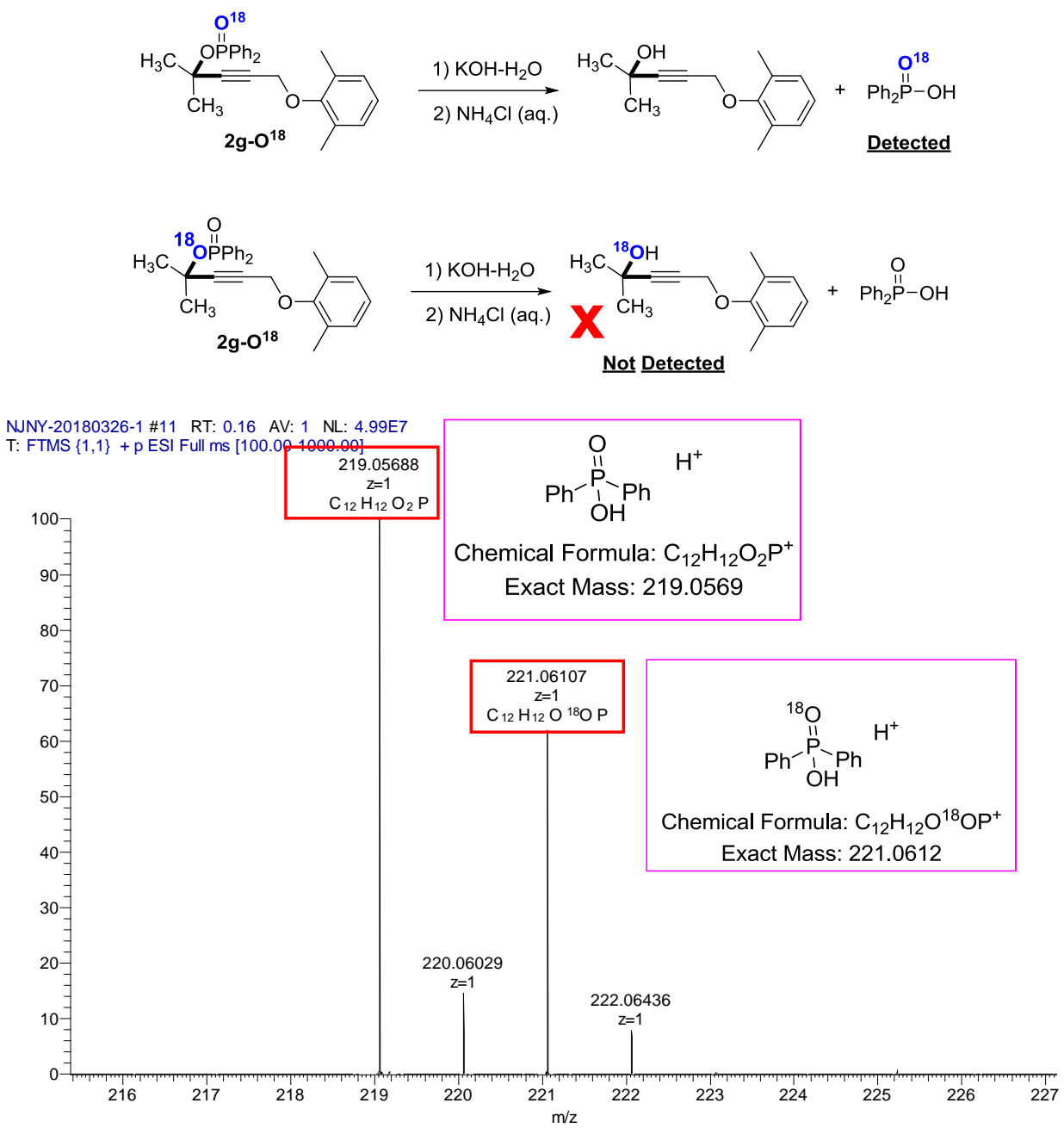

Figure S3. HRMS analysis of basic hydrolyzed ${ }^{18} \mathrm{O}$-labeling product 


\section{Determination of Singlet Oxygen Species}

\subsection{EPR Study}

To further explore the active species of singlet oxygen involved during the reaction, 2,2,6,6tetramethylpiperidine (TEMP) were used to trap ${ }^{1} \mathrm{O}_{2}$. Irradiation of DMF solution of TEMP, allenylphosphine oxide (1g), erythrosin B and 1-hydroxy-2-oxa-1-ioda(III)indan-3-one in air under $9 \mathrm{~W}$ White LED irradiation resulted in the formation of a strong characteristic signal of ${ }^{1} \mathrm{O}_{2}$ adduct with TEMP. $\left({ }^{1} \mathrm{O}_{2}, \mathrm{~g}=2.0064\right.$, ao $\left.=15.84 \mathrm{G}\right)$

Ref.: Zhao, L.; Li, P.; Xie, X.; Wang, L. Selective Remote C-H Trifluoromethylation of Aminoquinolines with $\mathrm{CF}_{3} \mathrm{SO}_{2} \mathrm{Na}$ under Visible Light Irradiation in the Absence of an External Photocatalyst, Org. Chem. Front. 2018, 5, 1689-1697. In this paper, the characterization data of singlet oxygen: ${ }^{1} \mathrm{O}_{2}, \mathrm{~g}=2.0070, \mathrm{a}_{\mathrm{O}}=15.79 \mathrm{G}$.

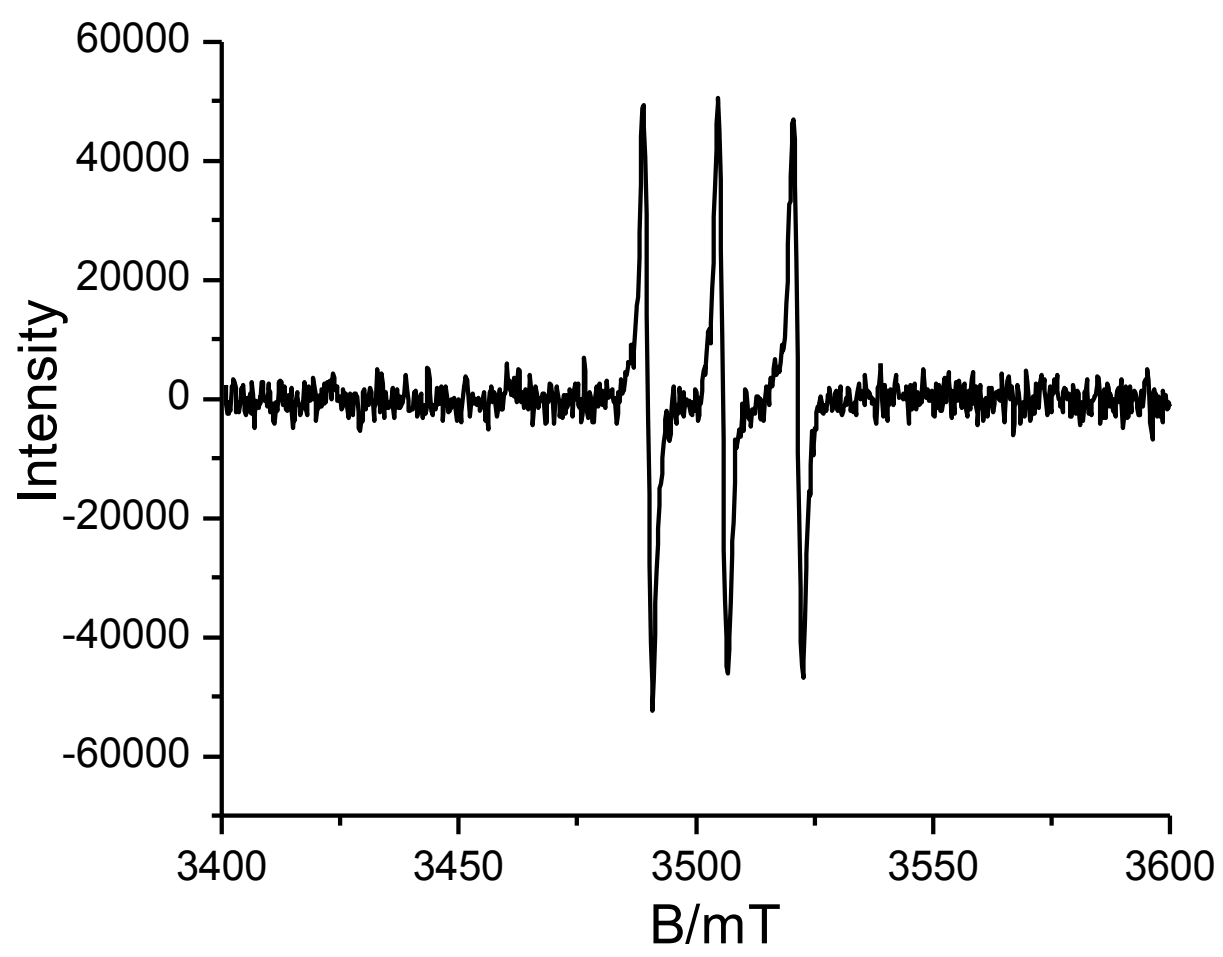

Figure S4. Electron spin resonance spectra of ${ }^{1} \mathrm{O}_{2}$ adduct with TEMP 


\subsection{Trapping Experiment}

We carried out control experiments trying to verify the presence of photoexcited singlet oxygen. Trapping experiment using 9,10-diphenylanthracene under the standard conditions afforded 9,10diphenyl-9,10-dihydro-9,10-epidioxyanthracene (3) in 69\% yield. The parallel experiment without BI-OH furnished 3 in $66 \%$ yield.

For the trapping of singlet oxygen with 9,10-dimethyl-9,10-dihydro-9,10-epidioxyanthracene, see: Ding, W.; Lu, L.; Zhou, Q.; Wei, Y.; Chen, J.; Xiao, W.-J. Bifunctional Photocatalysts for Enantioselective Aerobic Oxidation of $\beta$-Ketoesters, J. Am. Chem. Soc. 2017, 139, 63-66.<smiles>c1ccc(-c2c3ccccc3c(-c3ccccc3)c3ccccc23)cc1</smiles>
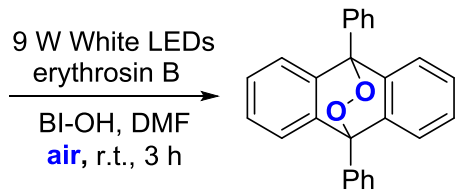

$3,69 \%$ yield (w/o BI-OH: 66\%)

\section{9,10-diphenyl-9,10-dihydro-9,10-epidioxyanthracene (3)}<smiles>OC(c1ccccc1)c1ccccc1-c1ccccc1-c1ccccc1</smiles>

A light white powder. ${ }^{1} \mathbf{H}$ NMR $\left(400 \mathrm{MHz}, \mathrm{CDCl}_{3}\right) \delta$ 7.73-7.68 (m, 4H), $7.63(\mathrm{t}, J=$ $7.6 \mathrm{~Hz}, 4 \mathrm{H}), 7.54(\mathrm{t}, J=7.3 \mathrm{~Hz}, 2 \mathrm{H}), 7.24-7.12(\mathrm{~m}, 8 \mathrm{H}) .{ }^{13} \mathbf{C}$ NMR $\left(101 \mathrm{MHz}, \mathrm{CDCl}_{3}\right) \delta 140.2$, 133.0, 128.4, 128.3, 127.7, 127.5, 123.5, 84.1. HR-MS: $[\mathrm{M}+\mathrm{H}]^{+}, \mathrm{C}_{26} \mathrm{H}_{19} \mathrm{O}_{2}$ : Calcd. 363.1380, Found, 363.1389 .

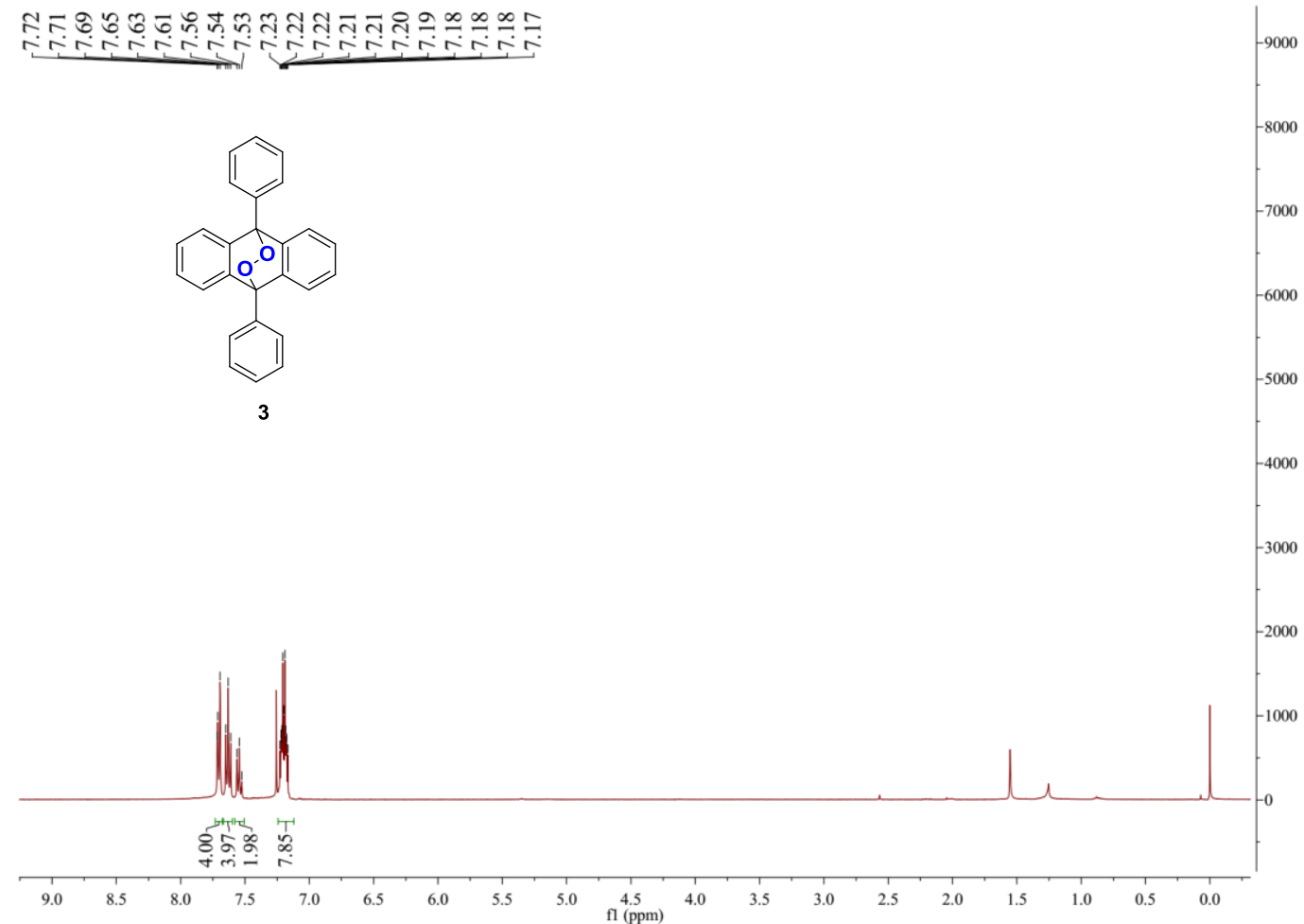



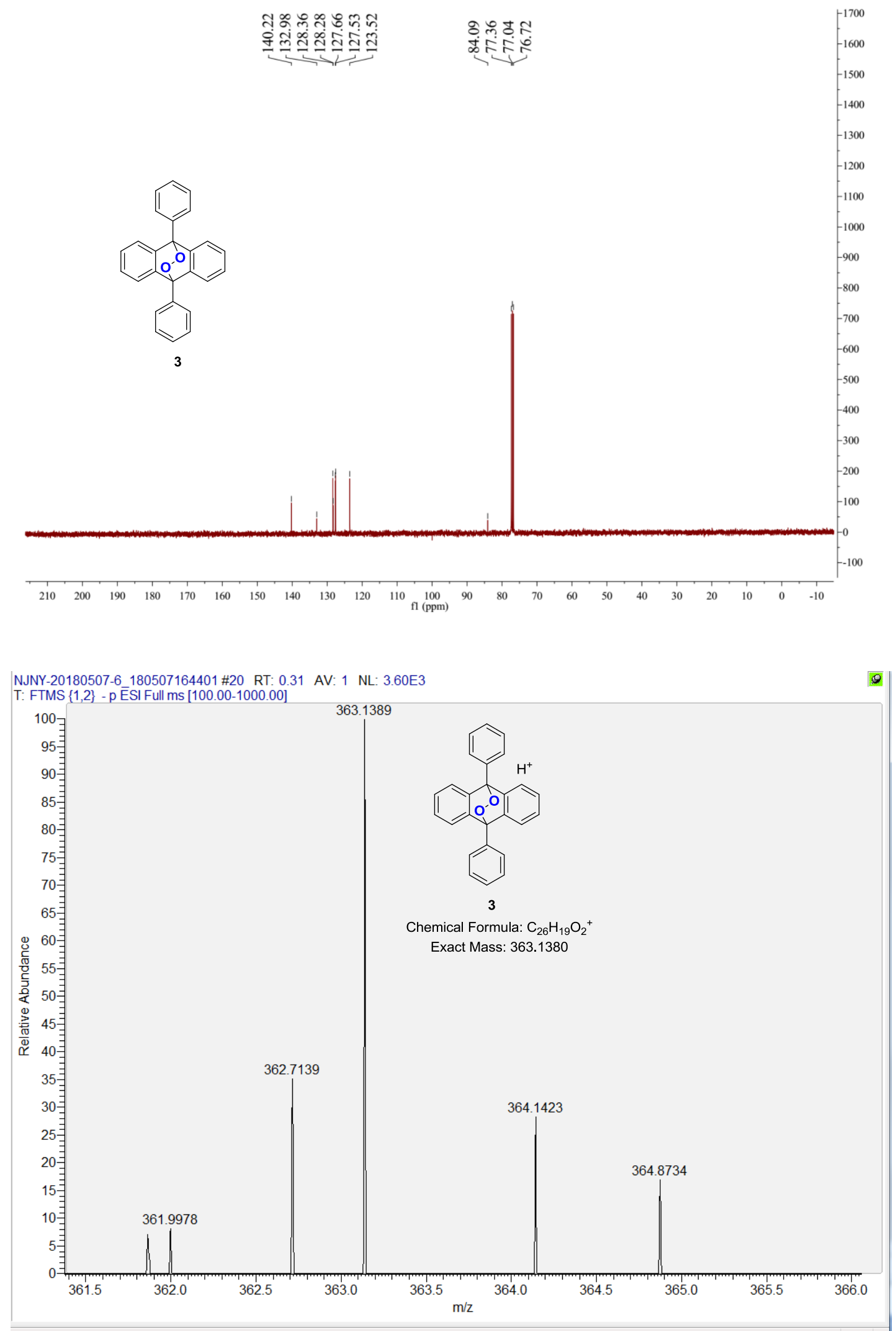

Figure S5. ${ }^{1} \mathrm{H}-\mathrm{NMR},{ }^{13} \mathrm{C}-\mathrm{NMR}$ and HR-MS of 3 


\section{Quenching Experiments of Erythrosin B}

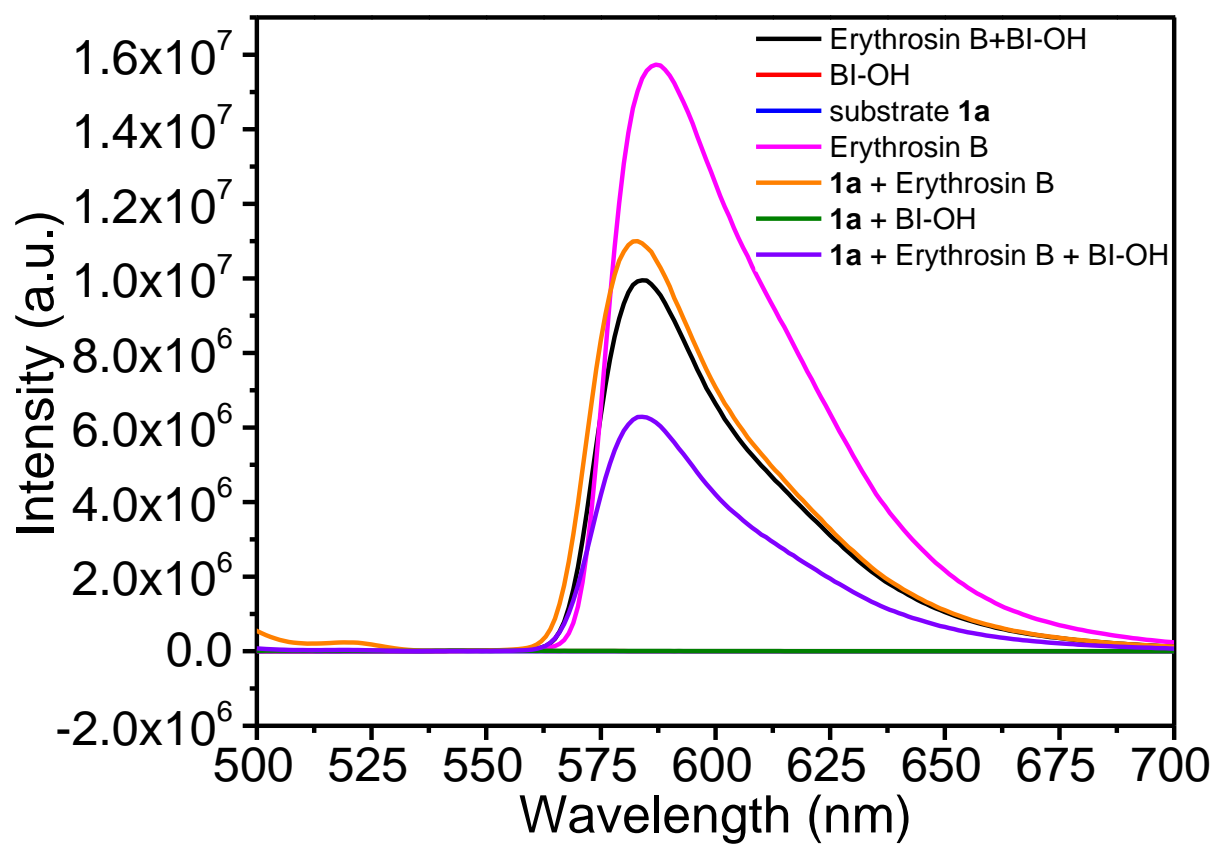

Figure S6. Fluorescence Quenching Experiments.

(Concentration: [erythrosin $\mathrm{B}]=0.2 \mathrm{mmol} / \mathrm{L} ;[\mathbf{1 a}]=2 \mathrm{mmol} / \mathrm{L} ;[\mathrm{BI}-\mathrm{OH}]=2 \mathrm{mmol} / \mathrm{L}$.)

\section{The Reaction Rate Curves With or Without of BI-OH}

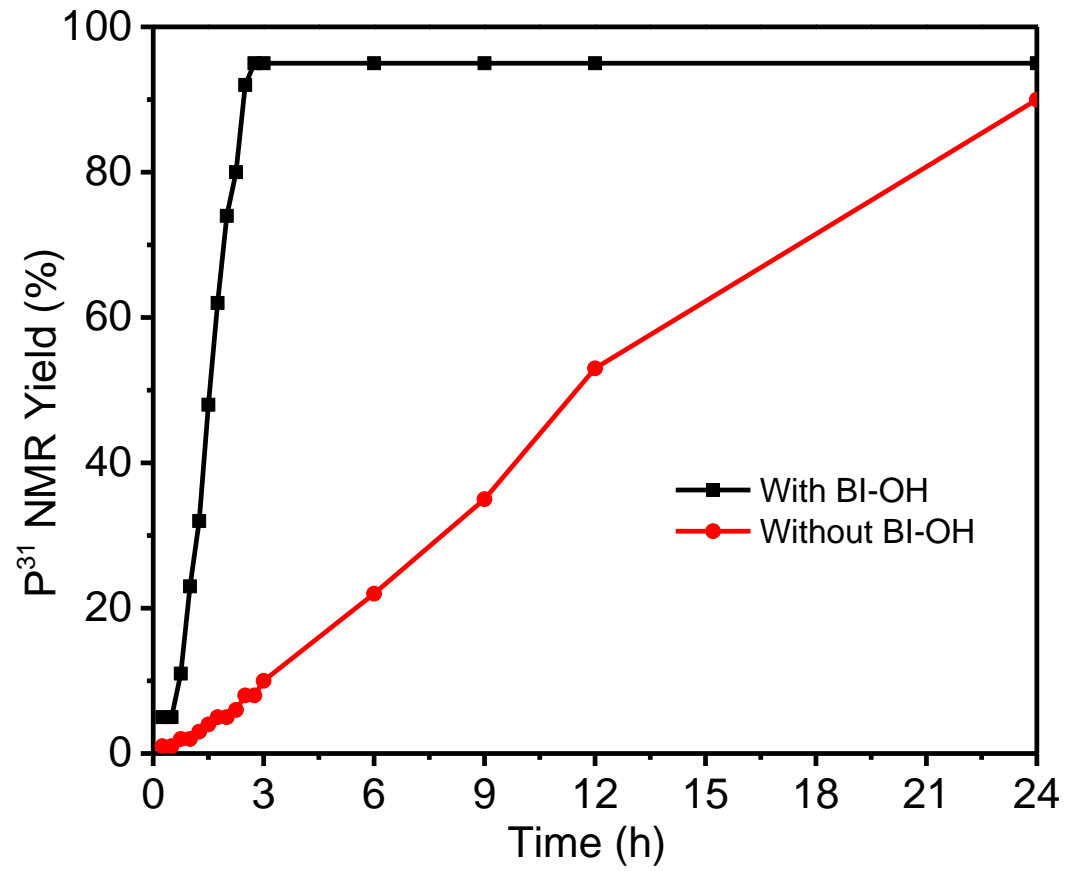

Figure S7. The influence of BI-OH on reaction rate (base on the yields of $\mathbf{2 a}$ ). 
11. X-Ray Crystallography Data of Compound 2b

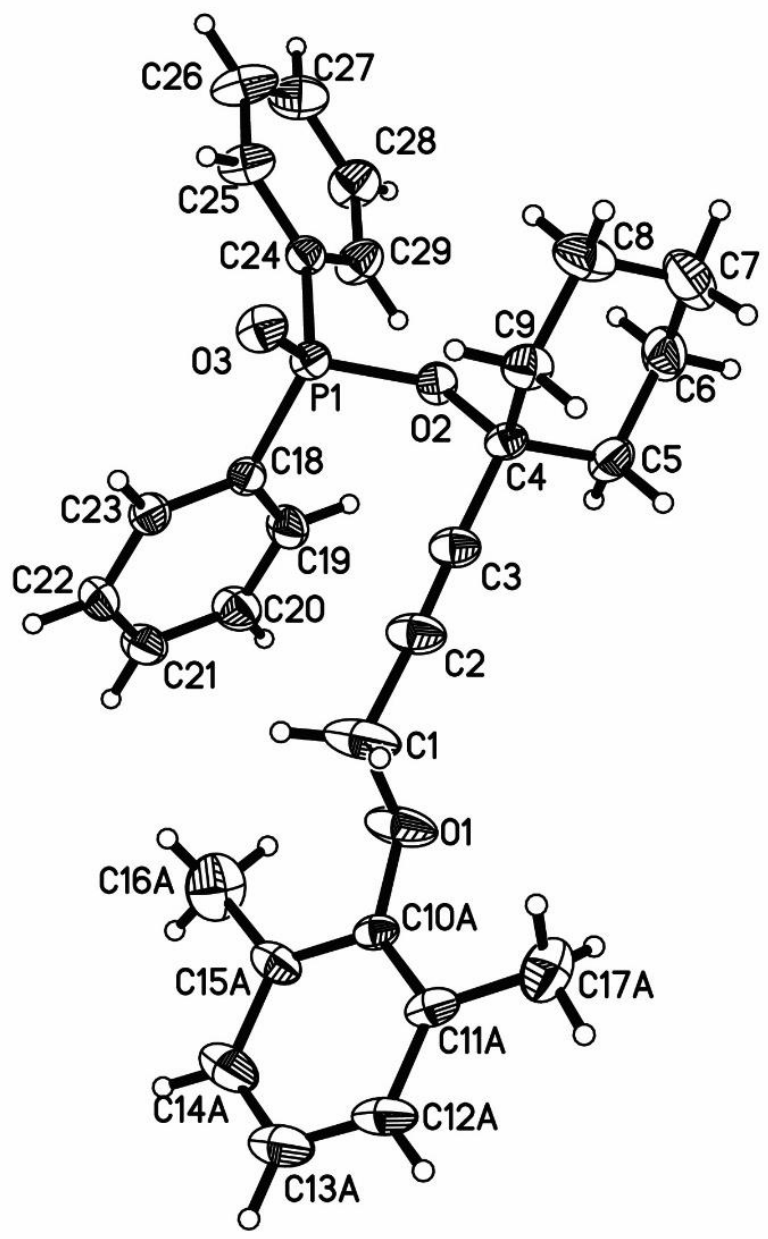

Table S4. Crystal data and structure refinement for $\mathbf{2 b}$.

Identification code

Empirical formula

Formula weight

Temperature

Wavelength

Crystal system

Space group

Unit cell dimensions

$108.9600(10)^{\circ}$

Volume

$\mathrm{Z}$

Density (calculated)

Absorption coefficient

$\mathrm{F}(000)$ 2b

C29 H31 O3 P

458.51

296(2) K

$0.71073 \AA$

Monoclinic

$\mathrm{P} 2{ }_{1} / \mathrm{n}$

$\mathrm{a}=16.4416(7) \AA$

$\alpha=90^{\circ}$.

$\mathrm{b}=8.8429(3) \AA$

$\beta=$

$\mathrm{c}=18.5563(8) \AA$

$\gamma=90^{\circ}$.

2551.55(18) $\AA^{3}$

4

$1.194 \mathrm{Mg} / \mathrm{m}^{3}$

$0.135 \mathrm{~mm}^{-1}$

976 
Crystal size

Theta range for data collection

Index ranges

Reflections collected

Independent reflections

Completeness to theta $=25.242^{\circ}$

Absorption correction

Max. and min. transmission

Refinement method

Data / restraints / parameters

Goodness-of-fit on $\mathrm{F}^{2}$

Final $\mathrm{R}$ indices [I $>2 \operatorname{sigma}(\mathrm{I})]$

$\mathrm{R}$ indices (all data)

Extinction coefficient

Largest diff. peak and hole
$0.230 \times 0.180 \times 0.160 \mathrm{~mm}^{3}$

2.321 to $27.507^{\circ}$.

$-21<=\mathrm{h}<=19,-11<=\mathrm{k}<=10,-24<=1<=22$

25700

$5849[\mathrm{R}(\mathrm{int})=0.0402]$

$99.6 \%$

Semi-empirical from equivalents

0.7456 and 0.6737

Full-matrix least-squares on $\mathrm{F}^{2}$

$5849 / 132 / 351$

1.046

$\mathrm{R} 1=0.0581, \mathrm{wR} 2=0.1469$

$\mathrm{R} 1=0.0922, \mathrm{wR} 2=0.1626$

$\mathrm{n} / \mathrm{a}$

0.250 and -0.291 e. $\AA^{-3}$ 
Table S5. Bond lengths $[\AA]$ and angles $\left[{ }^{\circ}\right]$ for $\mathbf{2 b}$.

\begin{tabular}{|c|c|}
\hline $\mathrm{C}(1)-\mathrm{O}(1)$ & $1.438(3)$ \\
\hline$C(1)-C(2)$ & $1.463(3)$ \\
\hline $\mathrm{C}(1)-\mathrm{H}(1 \mathrm{~A})$ & 0.9700 \\
\hline $\mathrm{C}(1)-\mathrm{H}(1 \mathrm{~B})$ & 0.9700 \\
\hline $\mathrm{C}(2)-\mathrm{C}(3)$ & $1.171(3)$ \\
\hline $\mathrm{C}(3)-\mathrm{C}(4)$ & $1.469(3)$ \\
\hline $\mathrm{C}(4)-\mathrm{O}(2)$ & $1.464(2)$ \\
\hline$C(4)-C(9)$ & $1.522(3)$ \\
\hline $\mathrm{C}(4)-\mathrm{C}(5)$ & $1.524(3)$ \\
\hline$C(5)-C(6)$ & $1.515(4)$ \\
\hline $\mathrm{C}(5)-\mathrm{H}(5 \mathrm{~A})$ & 0.9700 \\
\hline $\mathrm{C}(5)-\mathrm{H}(5 \mathrm{~B})$ & 0.9700 \\
\hline $\mathrm{C}(6)-\mathrm{C}(7)$ & $1.522(5)$ \\
\hline $\mathrm{C}(6)-\mathrm{H}(6 \mathrm{~A})$ & 0.9700 \\
\hline $\mathrm{C}(6)-\mathrm{H}(6 \mathrm{~B})$ & 0.9700 \\
\hline$C(7)-C(8)$ & $1.514(5)$ \\
\hline $\mathrm{C}(7)-\mathrm{H}(7 \mathrm{~A})$ & 0.9700 \\
\hline $\mathrm{C}(7)-\mathrm{H}(7 \mathrm{~B})$ & 0.9700 \\
\hline $\mathrm{C}(8)-\mathrm{C}(9)$ & $1.518(4)$ \\
\hline $\mathrm{C}(8)-\mathrm{H}(8 \mathrm{~A})$ & 0.9700 \\
\hline $\mathrm{C}(8)-\mathrm{H}(8 \mathrm{~B})$ & 0.9700 \\
\hline $\mathrm{C}(9)-\mathrm{H}(9 \mathrm{~A})$ & 0.9700 \\
\hline $\mathrm{C}(9)-\mathrm{H}(9 \mathrm{~B})$ & 0.9700 \\
\hline $\mathrm{C}(10 \mathrm{~A})-\mathrm{O}(1)$ & $1.369(4)$ \\
\hline$C(10 A)-C(11 A)$ & 1.3900 \\
\hline$C(10 A)-C(15 A)$ & 1.3900 \\
\hline$C(11 A)-C(12 A)$ & 1.3900 \\
\hline$C(11 \mathrm{~A})-\mathrm{C}(17 \mathrm{~A})$ & $1.495(9)$ \\
\hline $\mathrm{C}(12 \mathrm{~A})-\mathrm{C}(13 \mathrm{~A})$ & 1.3900 \\
\hline $\mathrm{C}(12 \mathrm{~A})-\mathrm{H}(12 \mathrm{~A})$ & 0.9300 \\
\hline$C(13 A)-C(14 A)$ & 1.3900 \\
\hline $\mathrm{C}(13 \mathrm{~A})-\mathrm{H}(13 \mathrm{~A})$ & 0.9300 \\
\hline$C(14 A)-C(15 A)$ & 1.3900 \\
\hline $\mathrm{C}(14 \mathrm{~A})-\mathrm{H}(14 \mathrm{~A})$ & 0.9300 \\
\hline$C(15 A)-C(16 A)$ & $1.547(9)$ \\
\hline $\mathrm{C}(16 \mathrm{~A})-\mathrm{H}(16 \mathrm{~A})$ & 0.9600 \\
\hline
\end{tabular}




\begin{tabular}{|c|c|}
\hline $\mathrm{C}(16 \mathrm{~A})-\mathrm{H}(16 \mathrm{~B})$ & 0.9600 \\
\hline $\mathrm{C}(16 \mathrm{~A})-\mathrm{H}(16 \mathrm{C})$ & 0.9600 \\
\hline $\mathrm{C}(17 \mathrm{~A})-\mathrm{H}(17 \mathrm{~A})$ & 0.9600 \\
\hline $\mathrm{C}(17 \mathrm{~A})-\mathrm{H}(17 \mathrm{~B})$ & 0.9600 \\
\hline $\mathrm{C}(17 \mathrm{~A})-\mathrm{H}(17 \mathrm{C})$ & 0.9600 \\
\hline $\mathrm{C}(10 \mathrm{~B})-\mathrm{O}(1)$ & $1.344(6)$ \\
\hline$C(10 B)-C(11 B)$ & 1.3900 \\
\hline$C(10 B)-C(15 B)$ & 1.3900 \\
\hline $\mathrm{C}(11 \mathrm{~B})-\mathrm{C}(12 \mathrm{~B})$ & 1.3900 \\
\hline $\mathrm{C}(11 \mathrm{~B})-\mathrm{C}(17 \mathrm{~B})$ & $1.562(10)$ \\
\hline $\mathrm{C}(12 \mathrm{~B})-\mathrm{C}(13 \mathrm{~B})$ & 1.3900 \\
\hline $\mathrm{C}(12 \mathrm{~B})-\mathrm{H}(12 \mathrm{~B})$ & 0.9300 \\
\hline $\mathrm{C}(13 \mathrm{~B})-\mathrm{C}(14 \mathrm{~B})$ & 1.3900 \\
\hline $\mathrm{C}(13 \mathrm{~B})-\mathrm{H}(13 \mathrm{~B})$ & 0.9300 \\
\hline $\mathrm{C}(14 \mathrm{~B})-\mathrm{C}(15 \mathrm{~B})$ & 1.3900 \\
\hline $\mathrm{C}(14 \mathrm{~B})-\mathrm{H}(14 \mathrm{~B})$ & 0.9300 \\
\hline$C(15 B)-C(16 B)$ & $1.473(13)$ \\
\hline $\mathrm{C}(16 \mathrm{~B})-\mathrm{H}(16 \mathrm{D})$ & 0.9600 \\
\hline $\mathrm{C}(16 \mathrm{~B})-\mathrm{H}(16 \mathrm{E})$ & 0.9600 \\
\hline $\mathrm{C}(16 \mathrm{~B})-\mathrm{H}(16 \mathrm{~F})$ & 0.9600 \\
\hline $\mathrm{C}(17 \mathrm{~B})-\mathrm{H}(17 \mathrm{D})$ & 0.9600 \\
\hline $\mathrm{C}(17 \mathrm{~B})-\mathrm{H}(17 \mathrm{E})$ & 0.9600 \\
\hline $\mathrm{C}(17 \mathrm{~B})-\mathrm{H}(17 \mathrm{~F})$ & 0.9600 \\
\hline $\mathrm{C}(18)-\mathrm{C}(19)$ & $1.390(3)$ \\
\hline $\mathrm{C}(18)-\mathrm{C}(23)$ & $1.391(3)$ \\
\hline $\mathrm{C}(18)-\mathrm{P}(1)$ & $1.797(2)$ \\
\hline C(19)-C(20) & $1.376(4)$ \\
\hline $\mathrm{C}(19)-\mathrm{H}(19)$ & 0.9300 \\
\hline $\mathrm{C}(20)-\mathrm{C}(21)$ & $1.374(4)$ \\
\hline $\mathrm{C}(20)-\mathrm{H}(20)$ & 0.9300 \\
\hline$C(21)-C(22)$ & $1.372(4)$ \\
\hline $\mathrm{C}(21)-\mathrm{H}(21)$ & 0.9300 \\
\hline$C(22)-C(23)$ & $1.380(4)$ \\
\hline $\mathrm{C}(22)-\mathrm{H}(22)$ & 0.9300 \\
\hline $\mathrm{C}(23)-\mathrm{H}(23)$ & 0.9300 \\
\hline$C(24)-C(29)$ & $1.376(3)$ \\
\hline$C(24)-C(25)$ & $1.379(4)$ \\
\hline $\mathrm{C}(24)-\mathrm{P}(1)$ & $1.800(2)$ \\
\hline
\end{tabular}




\begin{tabular}{|c|c|}
\hline$C(25)-C(26)$ & $1.379(4)$ \\
\hline $\mathrm{C}(25)-\mathrm{H}(25)$ & 0.9300 \\
\hline$C(26)-C(27)$ & $1.360(4)$ \\
\hline $\mathrm{C}(26)-\mathrm{H}(26)$ & 0.9300 \\
\hline $\mathrm{C}(27)-\mathrm{C}(28)$ & $1.366(4)$ \\
\hline $\mathrm{C}(27)-\mathrm{H}(27)$ & 0.9300 \\
\hline $\mathrm{C}(28)-\mathrm{C}(29)$ & $1.377(4)$ \\
\hline $\mathrm{C}(28)-\mathrm{H}(28)$ & 0.9300 \\
\hline $\mathrm{C}(29)-\mathrm{H}(29)$ & 0.9300 \\
\hline $\mathrm{O}(2)-\mathrm{P}(1)$ & $1.5865(16)$ \\
\hline $\mathrm{O}(3)-\mathrm{P}(1)$ & $1.4676(17)$ \\
\hline $\mathrm{O}(1)-\mathrm{C}(1)-\mathrm{C}(2)$ & $109.5(2)$ \\
\hline $\mathrm{O}(1)-\mathrm{C}(1)-\mathrm{H}(1 \mathrm{~A})$ & 109.8 \\
\hline $\mathrm{C}(2)-\mathrm{C}(1)-\mathrm{H}(1 \mathrm{~A})$ & 109.8 \\
\hline $\mathrm{O}(1)-\mathrm{C}(1)-\mathrm{H}(1 \mathrm{~B})$ & 109.8 \\
\hline $\mathrm{C}(2)-\mathrm{C}(1)-\mathrm{H}(1 \mathrm{~B})$ & 109.8 \\
\hline $\mathrm{H}(1 \mathrm{~A})-\mathrm{C}(1)-\mathrm{H}(1 \mathrm{~B})$ & 108.2 \\
\hline $\mathrm{C}(3)-\mathrm{C}(2)-\mathrm{C}(1)$ & $175.9(3)$ \\
\hline $\mathrm{C}(2)-\mathrm{C}(3)-\mathrm{C}(4)$ & $177.6(3)$ \\
\hline $\mathrm{O}(2)-\mathrm{C}(4)-\mathrm{C}(3)$ & $110.41(18)$ \\
\hline $\mathrm{O}(2)-\mathrm{C}(4)-\mathrm{C}(9)$ & $109.48(18)$ \\
\hline $\mathrm{C}(3)-\mathrm{C}(4)-\mathrm{C}(9)$ & $110.75(19)$ \\
\hline $\mathrm{O}(2)-\mathrm{C}(4)-\mathrm{C}(5)$ & 103.77(17) \\
\hline $\mathrm{C}(3)-\mathrm{C}(4)-\mathrm{C}(5)$ & 111.19(19) \\
\hline $\mathrm{C}(9)-\mathrm{C}(4)-\mathrm{C}(5)$ & $111.0(2)$ \\
\hline$C(6)-C(5)-C(4)$ & $111.8(2)$ \\
\hline $\mathrm{C}(6)-\mathrm{C}(5)-\mathrm{H}(5 \mathrm{~A})$ & 109.3 \\
\hline $\mathrm{C}(4)-\mathrm{C}(5)-\mathrm{H}(5 \mathrm{~A})$ & 109.3 \\
\hline $\mathrm{C}(6)-\mathrm{C}(5)-\mathrm{H}(5 \mathrm{~B})$ & 109.3 \\
\hline $\mathrm{C}(4)-\mathrm{C}(5)-\mathrm{H}(5 \mathrm{~B})$ & 109.3 \\
\hline $\mathrm{H}(5 \mathrm{~A})-\mathrm{C}(5)-\mathrm{H}(5 \mathrm{~B})$ & 107.9 \\
\hline$C(5)-C(6)-C(7)$ & $110.9(2)$ \\
\hline $\mathrm{C}(5)-\mathrm{C}(6)-\mathrm{H}(6 \mathrm{~A})$ & 109.4 \\
\hline $\mathrm{C}(7)-\mathrm{C}(6)-\mathrm{H}(6 \mathrm{~A})$ & 109.4 \\
\hline $\mathrm{C}(5)-\mathrm{C}(6)-\mathrm{H}(6 \mathrm{~B})$ & 109.4 \\
\hline $\mathrm{C}(7)-\mathrm{C}(6)-\mathrm{H}(6 \mathrm{~B})$ & 109.4 \\
\hline $\mathrm{H}(6 \mathrm{~A})-\mathrm{C}(6)-\mathrm{H}(6 \mathrm{~B})$ & 108.0 \\
\hline
\end{tabular}




\begin{tabular}{|c|c|}
\hline $\mathrm{C}(8)-\mathrm{C}(7)-\mathrm{C}(6)$ & $111.2(2)$ \\
\hline $\mathrm{C}(8)-\mathrm{C}(7)-\mathrm{H}(7 \mathrm{~A})$ & 109.4 \\
\hline $\mathrm{C}(6)-\mathrm{C}(7)-\mathrm{H}(7 \mathrm{~A})$ & 109.4 \\
\hline $\mathrm{C}(8)-\mathrm{C}(7)-\mathrm{H}(7 \mathrm{~B})$ & 109.4 \\
\hline $\mathrm{C}(6)-\mathrm{C}(7)-\mathrm{H}(7 \mathrm{~B})$ & 109.4 \\
\hline $\mathrm{H}(7 \mathrm{~A})-\mathrm{C}(7)-\mathrm{H}(7 \mathrm{~B})$ & 108.0 \\
\hline $\mathrm{C}(7)-\mathrm{C}(8)-\mathrm{C}(9)$ & $111.8(3)$ \\
\hline $\mathrm{C}(7)-\mathrm{C}(8)-\mathrm{H}(8 \mathrm{~A})$ & 109.2 \\
\hline $\mathrm{C}(9)-\mathrm{C}(8)-\mathrm{H}(8 \mathrm{~A})$ & 109.2 \\
\hline $\mathrm{C}(7)-\mathrm{C}(8)-\mathrm{H}(8 \mathrm{~B})$ & 109.2 \\
\hline $\mathrm{C}(9)-\mathrm{C}(8)-\mathrm{H}(8 \mathrm{~B})$ & 109.2 \\
\hline $\mathrm{H}(8 \mathrm{~A})-\mathrm{C}(8)-\mathrm{H}(8 \mathrm{~B})$ & 107.9 \\
\hline $\mathrm{C}(8)-\mathrm{C}(9)-\mathrm{C}(4)$ & $111.4(2)$ \\
\hline $\mathrm{C}(8)-\mathrm{C}(9)-\mathrm{H}(9 \mathrm{~A})$ & 109.3 \\
\hline $\mathrm{C}(4)-\mathrm{C}(9)-\mathrm{H}(9 \mathrm{~A})$ & 109.3 \\
\hline $\mathrm{C}(8)-\mathrm{C}(9)-\mathrm{H}(9 \mathrm{~B})$ & 109.3 \\
\hline $\mathrm{C}(4)-\mathrm{C}(9)-\mathrm{H}(9 \mathrm{~B})$ & 109.3 \\
\hline $\mathrm{H}(9 \mathrm{~A})-\mathrm{C}(9)-\mathrm{H}(9 \mathrm{~B})$ & 108.0 \\
\hline $\mathrm{O}(1)-\mathrm{C}(10 \mathrm{~A})-\mathrm{C}(11 \mathrm{~A})$ & $132.0(6)$ \\
\hline $\mathrm{O}(1)-\mathrm{C}(10 \mathrm{~A})-\mathrm{C}(15 \mathrm{~A})$ & $108.0(6)$ \\
\hline$C(11 A)-C(10 A)-C(15 A)$ & 120.0 \\
\hline$C(10 A)-C(11 A)-C(12 A)$ & 120.0 \\
\hline$C(10 A)-C(11 A)-C(17 A)$ & $117.5(5)$ \\
\hline$C(12 A)-C(11 A)-C(17 A)$ & $122.5(5)$ \\
\hline$C(13 A)-C(12 A)-C(11 A)$ & 120.0 \\
\hline $\mathrm{C}(13 \mathrm{~A})-\mathrm{C}(12 \mathrm{~A})-\mathrm{H}(12 \mathrm{~A})$ & 120.0 \\
\hline $\mathrm{C}(11 \mathrm{~A})-\mathrm{C}(12 \mathrm{~A})-\mathrm{H}(12 \mathrm{~A})$ & 120.0 \\
\hline$C(14 A)-C(13 A)-C(12 A)$ & 120.0 \\
\hline $\mathrm{C}(14 \mathrm{~A})-\mathrm{C}(13 \mathrm{~A})-\mathrm{H}(13 \mathrm{~A})$ & 120.0 \\
\hline $\mathrm{C}(12 \mathrm{~A})-\mathrm{C}(13 \mathrm{~A})-\mathrm{H}(13 \mathrm{~A})$ & 120.0 \\
\hline$C(13 A)-C(14 A)-C(15 A)$ & 120.0 \\
\hline $\mathrm{C}(13 \mathrm{~A})-\mathrm{C}(14 \mathrm{~A})-\mathrm{H}(14 \mathrm{~A})$ & 120.0 \\
\hline $\mathrm{C}(15 \mathrm{~A})-\mathrm{C}(14 \mathrm{~A})-\mathrm{H}(14 \mathrm{~A})$ & 120.0 \\
\hline$C(14 A)-C(15 A)-C(10 A)$ & 120.0 \\
\hline$C(14 A)-C(15 A)-C(16 A)$ & $117.4(5)$ \\
\hline$C(10 A)-C(15 A)-C(16 A)$ & $122.6(5)$ \\
\hline$C(15 A)-C(16 A)-H(16 A)$ & 109.5 \\
\hline $\mathrm{C}(15 \mathrm{~A})-\mathrm{C}(16 \mathrm{~A})-\mathrm{H}(16 \mathrm{~B})$ & 109.5 \\
\hline
\end{tabular}




\begin{tabular}{|c|c|}
\hline$H(16 A)-C(16 A)-H(16 B)$ & 109.5 \\
\hline $\mathrm{C}(15 \mathrm{~A})-\mathrm{C}(16 \mathrm{~A})-\mathrm{H}(16 \mathrm{C})$ & 109.5 \\
\hline $\mathrm{H}(16 \mathrm{~A})-\mathrm{C}(16 \mathrm{~A})-\mathrm{H}(16 \mathrm{C})$ & 109.5 \\
\hline $\mathrm{H}(16 \mathrm{~B})-\mathrm{C}(16 \mathrm{~A})-\mathrm{H}(16 \mathrm{C})$ & 109.5 \\
\hline $\mathrm{C}(11 \mathrm{~A})-\mathrm{C}(17 \mathrm{~A})-\mathrm{H}(17 \mathrm{~A})$ & 109.5 \\
\hline $\mathrm{C}(11 \mathrm{~A})-\mathrm{C}(17 \mathrm{~A})-\mathrm{H}(17 \mathrm{~B})$ & 109.5 \\
\hline $\mathrm{H}(17 \mathrm{~A})-\mathrm{C}(17 \mathrm{~A})-\mathrm{H}(17 \mathrm{~B})$ & 109.5 \\
\hline $\mathrm{C}(11 \mathrm{~A})-\mathrm{C}(17 \mathrm{~A})-\mathrm{H}(17 \mathrm{C})$ & 109.5 \\
\hline $\mathrm{H}(17 \mathrm{~A})-\mathrm{C}(17 \mathrm{~A})-\mathrm{H}(17 \mathrm{C})$ & 109.5 \\
\hline $\mathrm{H}(17 \mathrm{~B})-\mathrm{C}(17 \mathrm{~A})-\mathrm{H}(17 \mathrm{C})$ & 109.5 \\
\hline $\mathrm{O}(1)-\mathrm{C}(10 \mathrm{~B})-\mathrm{C}(11 \mathrm{~B})$ & $100.9(8)$ \\
\hline $\mathrm{O}(1)-\mathrm{C}(10 \mathrm{~B})-\mathrm{C}(15 \mathrm{~B})$ & 139.1(8) \\
\hline $\mathrm{C}(11 \mathrm{~B})-\mathrm{C}(10 \mathrm{~B})-\mathrm{C}(15 \mathrm{~B})$ & 120.0 \\
\hline $\mathrm{C}(10 \mathrm{~B})-\mathrm{C}(11 \mathrm{~B})-\mathrm{C}(12 \mathrm{~B})$ & 120.0 \\
\hline $\mathrm{C}(10 \mathrm{~B})-\mathrm{C}(11 \mathrm{~B})-\mathrm{C}(17 \mathrm{~B})$ & $122.6(7)$ \\
\hline $\mathrm{C}(12 \mathrm{~B})-\mathrm{C}(11 \mathrm{~B})-\mathrm{C}(17 \mathrm{~B})$ & $117.4(7)$ \\
\hline $\mathrm{C}(13 \mathrm{~B})-\mathrm{C}(12 \mathrm{~B})-\mathrm{C}(11 \mathrm{~B})$ & 120.0 \\
\hline $\mathrm{C}(13 \mathrm{~B})-\mathrm{C}(12 \mathrm{~B})-\mathrm{H}(12 \mathrm{~B})$ & 120.0 \\
\hline $\mathrm{C}(11 \mathrm{~B})-\mathrm{C}(12 \mathrm{~B})-\mathrm{H}(12 \mathrm{~B})$ & 120.0 \\
\hline $\mathrm{C}(12 \mathrm{~B})-\mathrm{C}(13 \mathrm{~B})-\mathrm{C}(14 \mathrm{~B})$ & 120.0 \\
\hline $\mathrm{C}(12 \mathrm{~B})-\mathrm{C}(13 \mathrm{~B})-\mathrm{H}(13 \mathrm{~B})$ & 120.0 \\
\hline $\mathrm{C}(14 \mathrm{~B})-\mathrm{C}(13 \mathrm{~B})-\mathrm{H}(13 \mathrm{~B})$ & 120.0 \\
\hline $\mathrm{C}(13 \mathrm{~B})-\mathrm{C}(14 \mathrm{~B})-\mathrm{C}(15 \mathrm{~B})$ & 120.0 \\
\hline $\mathrm{C}(13 \mathrm{~B})-\mathrm{C}(14 \mathrm{~B})-\mathrm{H}(14 \mathrm{~B})$ & 120.0 \\
\hline $\mathrm{C}(15 \mathrm{~B})-\mathrm{C}(14 \mathrm{~B})-\mathrm{H}(14 \mathrm{~B})$ & 120.0 \\
\hline $\mathrm{C}(14 \mathrm{~B})-\mathrm{C}(15 \mathrm{~B})-\mathrm{C}(10 \mathrm{~B})$ & 120.0 \\
\hline $\mathrm{C}(14 \mathrm{~B})-\mathrm{C}(15 \mathrm{~B})-\mathrm{C}(16 \mathrm{~B})$ & $123.9(7)$ \\
\hline $\mathrm{C}(10 \mathrm{~B})-\mathrm{C}(15 \mathrm{~B})-\mathrm{C}(16 \mathrm{~B})$ & $116.1(7)$ \\
\hline $\mathrm{C}(15 \mathrm{~B})-\mathrm{C}(16 \mathrm{~B})-\mathrm{H}(16 \mathrm{D})$ & 109.5 \\
\hline $\mathrm{C}(15 \mathrm{~B})-\mathrm{C}(16 \mathrm{~B})-\mathrm{H}(16 \mathrm{E})$ & 109.5 \\
\hline $\mathrm{H}(16 \mathrm{D})-\mathrm{C}(16 \mathrm{~B})-\mathrm{H}(16 \mathrm{E})$ & 109.5 \\
\hline $\mathrm{C}(15 \mathrm{~B})-\mathrm{C}(16 \mathrm{~B})-\mathrm{H}(16 \mathrm{~F})$ & 109.5 \\
\hline $\mathrm{H}(16 \mathrm{D})-\mathrm{C}(16 \mathrm{~B})-\mathrm{H}(16 \mathrm{~F})$ & 109.5 \\
\hline $\mathrm{H}(16 \mathrm{E})-\mathrm{C}(16 \mathrm{~B})-\mathrm{H}(16 \mathrm{~F})$ & 109.5 \\
\hline $\mathrm{C}(11 \mathrm{~B})-\mathrm{C}(17 \mathrm{~B})-\mathrm{H}(17 \mathrm{D})$ & 109.5 \\
\hline $\mathrm{C}(11 \mathrm{~B})-\mathrm{C}(17 \mathrm{~B})-\mathrm{H}(17 \mathrm{E})$ & 109.5 \\
\hline H(17D)-C(17B)-H(17E) & 109.5 \\
\hline $\mathrm{C}(11 \mathrm{~B})-\mathrm{C}(17 \mathrm{~B})-\mathrm{H}(17 \mathrm{~F})$ & 109.5 \\
\hline
\end{tabular}




\begin{tabular}{|c|c|}
\hline $\mathrm{H}(17 \mathrm{D})-\mathrm{C}(17 \mathrm{~B})-\mathrm{H}(17 \mathrm{~F})$ & 109.5 \\
\hline $\mathrm{H}(17 \mathrm{E})-\mathrm{C}(17 \mathrm{~B})-\mathrm{H}(17 \mathrm{~F})$ & 109.5 \\
\hline $\mathrm{C}(19)-\mathrm{C}(18)-\mathrm{C}(23)$ & $118.3(2)$ \\
\hline $\mathrm{C}(19)-\mathrm{C}(18)-\mathrm{P}(1)$ & $122.75(17)$ \\
\hline $\mathrm{C}(23)-\mathrm{C}(18)-\mathrm{P}(1)$ & $118.97(18)$ \\
\hline $\mathrm{C}(20)-\mathrm{C}(19)-\mathrm{C}(18)$ & $120.8(2)$ \\
\hline $\mathrm{C}(20)-\mathrm{C}(19)-\mathrm{H}(19)$ & 119.6 \\
\hline $\mathrm{C}(18)-\mathrm{C}(19)-\mathrm{H}(19)$ & 119.6 \\
\hline $\mathrm{C}(21)-\mathrm{C}(20)-\mathrm{C}(19)$ & $120.0(3)$ \\
\hline $\mathrm{C}(21)-\mathrm{C}(20)-\mathrm{H}(20)$ & 120.0 \\
\hline $\mathrm{C}(19)-\mathrm{C}(20)-\mathrm{H}(20)$ & 120.0 \\
\hline $\mathrm{C}(22)-\mathrm{C}(21)-\mathrm{C}(20)$ & $120.1(2)$ \\
\hline $\mathrm{C}(22)-\mathrm{C}(21)-\mathrm{H}(21)$ & 119.9 \\
\hline $\mathrm{C}(20)-\mathrm{C}(21)-\mathrm{H}(21)$ & 119.9 \\
\hline $\mathrm{C}(21)-\mathrm{C}(22)-\mathrm{C}(23)$ & $120.2(2)$ \\
\hline $\mathrm{C}(21)-\mathrm{C}(22)-\mathrm{H}(22)$ & 119.9 \\
\hline $\mathrm{C}(23)-\mathrm{C}(22)-\mathrm{H}(22)$ & 119.9 \\
\hline $\mathrm{C}(22)-\mathrm{C}(23)-\mathrm{C}(18)$ & $120.6(2)$ \\
\hline $\mathrm{C}(22)-\mathrm{C}(23)-\mathrm{H}(23)$ & 119.7 \\
\hline $\mathrm{C}(18)-\mathrm{C}(23)-\mathrm{H}(23)$ & 119.7 \\
\hline $\mathrm{C}(29)-\mathrm{C}(24)-\mathrm{C}(25)$ & $118.4(2)$ \\
\hline $\mathrm{C}(29)-\mathrm{C}(24)-\mathrm{P}(1)$ & $122.73(18)$ \\
\hline $\mathrm{C}(25)-\mathrm{C}(24)-\mathrm{P}(1)$ & $118.90(19)$ \\
\hline $\mathrm{C}(26)-\mathrm{C}(25)-\mathrm{C}(24)$ & $120.5(3)$ \\
\hline $\mathrm{C}(26)-\mathrm{C}(25)-\mathrm{H}(25)$ & 119.7 \\
\hline $\mathrm{C}(24)-\mathrm{C}(25)-\mathrm{H}(25)$ & 119.7 \\
\hline $\mathrm{C}(27)-\mathrm{C}(26)-\mathrm{C}(25)$ & $120.2(3)$ \\
\hline $\mathrm{C}(27)-\mathrm{C}(26)-\mathrm{H}(26)$ & 119.9 \\
\hline $\mathrm{C}(25)-\mathrm{C}(26)-\mathrm{H}(26)$ & 119.9 \\
\hline$C(26)-C(27)-C(28)$ & $120.2(3)$ \\
\hline $\mathrm{C}(26)-\mathrm{C}(27)-\mathrm{H}(27)$ & 119.9 \\
\hline $\mathrm{C}(28)-\mathrm{C}(27)-\mathrm{H}(27)$ & 119.9 \\
\hline $\mathrm{C}(27)-\mathrm{C}(28)-\mathrm{C}(29)$ & $119.7(3)$ \\
\hline $\mathrm{C}(27)-\mathrm{C}(28)-\mathrm{H}(28)$ & 120.2 \\
\hline $\mathrm{C}(29)-\mathrm{C}(28)-\mathrm{H}(28)$ & 120.2 \\
\hline $\mathrm{C}(24)-\mathrm{C}(29)-\mathrm{C}(28)$ & $121.0(3)$ \\
\hline $\mathrm{C}(24)-\mathrm{C}(29)-\mathrm{H}(29)$ & 119.5 \\
\hline $\mathrm{C}(28)-\mathrm{C}(29)-\mathrm{H}(29)$ & 119.5 \\
\hline
\end{tabular}




$\begin{array}{lc}\mathrm{C}(10 \mathrm{~B})-\mathrm{O}(1)-\mathrm{C}(1) & 110.9(6) \\ \mathrm{C}(10 \mathrm{~A})-\mathrm{O}(1)-\mathrm{C}(1) & 112.4(4) \\ \mathrm{C}(4)-\mathrm{O}(2)-\mathrm{P}(1) & 127.30(13) \\ \mathrm{O}(3)-\mathrm{P}(1)-\mathrm{O}(2) & 117.43(10) \\ \mathrm{O}(3)-\mathrm{P}(1)-\mathrm{C}(18) & 111.80(10) \\ \mathrm{O}(2)-\mathrm{P}(1)-\mathrm{C}(18) & 106.34(9) \\ \mathrm{O}(3)-\mathrm{P}(1)-\mathrm{C}(24) & 113.20(11) \\ \mathrm{O}(2)-\mathrm{P}(1)-\mathrm{C}(24) & 98.21(9) \\ \mathrm{C}(18)-\mathrm{P}(1)-\mathrm{C}(24) & 108.78(10)\end{array}$

Symmetry transformations used to generate equivalent atoms:

Table S6. Hydrogen bonds for $\mathbf{2 b}\left[\AA\right.$ and $\left.^{\circ}\right]$.

\begin{tabular}{lcccc}
\hline D-H...A & d(D-H) & d(H...A & $d($ D...A $)$ & $<($ DHA $)$ \\
\hline C(9)-H(9A)...O(3) & 0.97 & 2.60 & $3.278(3)$ & 127.3 \\
\hline
\end{tabular}

Symmetry transformations used to generate equivalent atoms: 


\section{Characterizations of Products:}

\section{1-(3-(2,6-dimethylphenoxy)prop-1-yn-1-yl)cycloheptyl diphenylphosphinate (2a)}

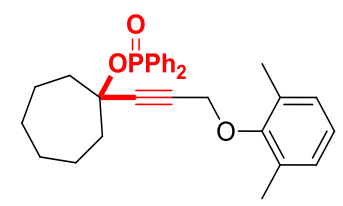

A white solid, m.p.: $91.4-91.9{ }^{\circ} \mathrm{C},(84.1 \mathrm{mg}, 89 \%$ yield $) .{ }^{1} \mathbf{H}$ NMR $\left(400 \mathrm{MHz}, \mathrm{CDCl}_{3}\right) \delta 7.86-$ $7.76(\mathrm{~m}, 4 \mathrm{H}), 7.49-7.36(\mathrm{~m}, 6 \mathrm{H}), 7.03-6.90(\mathrm{~m}, 3 \mathrm{H}), 4.13(\mathrm{~s}, 2 \mathrm{H}), 2.34-2.26(\mathrm{~m}, 4 \mathrm{H}), 2.22(\mathrm{~s}$, 6H), $1.75-1.65(\mathrm{~m}, 2 \mathrm{H}), 1.63-1.47(\mathrm{~m}, 6 \mathrm{H}) .{ }^{13} \mathbf{C} \mathbf{N M R}\left(101 \mathrm{MHz}, \mathrm{CDCl}_{3}\right) \delta$ 155.1, 134.7, 133.3, 131.5, 131.4, 131.2, 128.7, 128.3, 128.1, 124.2, 87.6 (d, $J=5.1 \mathrm{~Hz}), 84.2,81.3(\mathrm{~d}, J=9.1 \mathrm{~Hz}), 59.7$, $43.1(\mathrm{~d}, J=4.0 \mathrm{~Hz}), 28.0,21.8,16.6 .{ }^{31} \mathbf{P}$ NMR $\left(162 \mathrm{MHz}, \mathrm{CDCl}_{3}\right) \delta 27.28$ (s). HRMS (ESI): $\left([\mathrm{M}+\mathrm{Na}]^{+}\right)$Calcd for $\mathrm{C}_{30} \mathrm{H}_{33} \mathrm{NaO}_{3} \mathrm{P}: 495.2065$, Found: 495.2059. IR (film) $v$ 2932, 2859, 2160, 1439, $1228,1192,1161,966,726,697 \mathrm{~cm}^{-1}$.

1-(3-(2,6-dimethylphenoxy)prop-1-yn-1-yl)cyclohexyl diphenylphosphinate (2b)

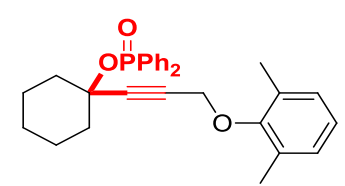

A white solid, m.p.: $97.5-98.7{ }^{\circ} \mathrm{C},(79.7 \mathrm{mg}, 87 \%$ yield $) .{ }^{1} \mathbf{H}$ NMR $\left(400 \mathrm{MHz}, \mathrm{CDCl}_{3}\right) \delta 7.90-$ $7.70(\mathrm{~m}, 4 \mathrm{H}), 7.56-7.33(\mathrm{~m}, 6 \mathrm{H}), 7.07-6.86(\mathrm{~m}, 3 \mathrm{H}), 4.15(\mathrm{~s}, 2 \mathrm{H}), 2.22(\mathrm{~s}, 6 \mathrm{H}), 2.17-2.03(\mathrm{~m}$, 4H), $1.75-1.65(\mathrm{~m}, 2 \mathrm{H}), 1.58-1.44(\mathrm{~m}, 3 \mathrm{H}), 1.37-1.32(\mathrm{~m}, 1 \mathrm{H}) .{ }^{13} \mathbf{C} \mathbf{~ N M R}\left(101 \mathrm{MHz}, \mathrm{CDCl}_{3}\right)$ $\delta 155.1,134.6,133.2,131.5,131.4,131.2,128.7,128.3,128.1,124.2,86.6(\mathrm{~d}, J=3.0 \mathrm{~Hz}), 84.7$, $78.1(\mathrm{~d}, J=3.6 \mathrm{~Hz}), 59.7,39.8(\mathrm{~d}, J=3.0 \mathrm{~Hz}), 24.8,22.9,16.6 .{ }^{31} \mathbf{P}$ NMR $\left(162 \mathrm{MHz}, \mathrm{CDCl}_{3}\right) \delta$ 27.63 (s). HRMS (ESI): ([M+Na] $]^{+}$) Calcd for $\mathrm{C}_{29} \mathrm{H}_{31} \mathrm{NaO}_{3} \mathrm{P}: 481.1909$, Found: 481.1903. IR (film) $v 2920,2850,1646,1437,1122,1060,953,725,690 \mathrm{~cm}^{-1}$.

1-(3-(2,6-dimethylphenoxy)prop-1-yn-1-yl)cyclopentyl diphenylphosphinate (2c)

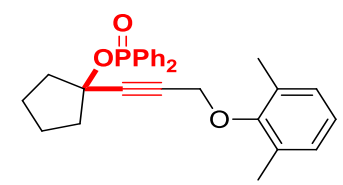

A pale yellow solid, m.p.: $116.1-117.0{ }^{\circ} \mathrm{C},(70.2 \mathrm{mg}, 79 \%$ yield $) .{ }^{1} \mathbf{H}$ NMR $\left(400 \mathrm{MHz}, \mathrm{CDCl}_{3}\right) \delta$ $7.86-7.72(\mathrm{~m}, 4 \mathrm{H}), 7.50-7.36(\mathrm{~m}, 6 \mathrm{H}), 7.07-6.88(\mathrm{~m}, 3 \mathrm{H}), 4.10(\mathrm{~s}, 2 \mathrm{H}), 2.57-2.48(\mathrm{~m}, 2 \mathrm{H})$, $2.22(\mathrm{~s}, 6 \mathrm{H}), 2.10-2.00(\mathrm{~m}, 2 \mathrm{H}), 1.97-1.85(\mathrm{~m}, 2 \mathrm{H}), 1.84-1.72(\mathrm{~m}, 2 \mathrm{H}) .{ }^{13} \mathbf{C}$ NMR $(101 \mathrm{MHz}$ 
$\left.\mathrm{CDCl}_{3}\right) \delta 155.1,134.2,132.8,131.6,131.6,131.6,131.5,131.2,128.7,128.2,128.1,124.2,86.5$, 83.9, $82.8(\mathrm{~d}, J=9.1 \mathrm{~Hz}), 59.6,42.4(\mathrm{~d}, J=5.1 \mathrm{~Hz}), 23.0,16.6 .{ }^{31} \mathbf{P}$ NMR $\left(162 \mathrm{MHz}, \mathrm{CDCl}_{3}\right) \delta$ 28.34 (s). HRMS (ESI): ([M+Na] $]^{+}$) Calcd for $\mathrm{C}_{28} \mathrm{H}_{29} \mathrm{NaO}_{3} \mathrm{P}: 467.1752$, Found: 467.1746. IR (film) $v 2919,2847,2158,1976,1436,1289,1139,1082,746,693 \mathrm{~cm}^{-1}$.

3-(3-(2,6-dimethylphenoxy)prop-1-yn-1-yl)tetrahydrothiophen-3-yl diphenylphosphinate (2d)

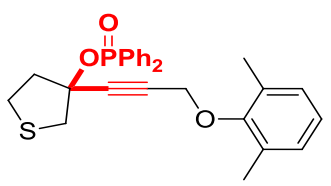

A white solid, m.p.: $116.8-117.5{ }^{\circ} \mathrm{C},(53.6 \mathrm{mg}, 58 \%$ yield $) .{ }^{1} \mathbf{H} \mathbf{N M R}\left(400 \mathrm{MHz}, \mathrm{CDCl}_{3}\right) \delta 7.87-$ $7.74(\mathrm{~m}, 4 \mathrm{H}), 7.54-7.38(\mathrm{~m}, 6 \mathrm{H}), 7.05-6.90(\mathrm{~m}, 3 \mathrm{H}), 4.10(\mathrm{~s}, 2 \mathrm{H}), 3.67-3.59(\mathrm{~m}, 1 \mathrm{H}), 3.25(\mathrm{~d}$, $J=8.0 \mathrm{~Hz}, 1 \mathrm{H}), 3.18-3.08(\mathrm{~m}, 1 \mathrm{H}), 3.05-2.97(\mathrm{~m}, 1 \mathrm{H}), 2.96-2.87(\mathrm{~m}, 1 \mathrm{H}), 2.31-2.22(\mathrm{~m}, 1 \mathrm{H})$, $2.20(\mathrm{~s}, 6 \mathrm{H}) .{ }^{13} \mathbf{C}$ NMR $\left(101 \mathrm{MHz}, \mathrm{CDCl}_{3}\right) \delta 154.9,133.6(\mathrm{~d}, J=18.2 \mathrm{~Hz}), 132.2(\mathrm{~d}, J=20.2 \mathrm{~Hz})$, $132.0(\mathrm{~d}, J=5.1 \mathrm{~Hz}), 131.9$ (d, $J=5.1 \mathrm{~Hz}), 131.6$ (d, $J=2.0 \mathrm{~Hz}), 131.5(\mathrm{~d}, J=2.0 \mathrm{~Hz}), 131.1$, 128.8, 128.4 (d, $J=6.1, \mathrm{~Hz}), 128.3(\mathrm{~d}, J=6.1 \mathrm{~Hz}), 124.4,85.3,83.2(\mathrm{~d}, J=3.0 \mathrm{~Hz}), 81.9$ (d, $J=$ $9.1 \mathrm{~Hz}), 59.4,44.7(\mathrm{~d}, J=4.0 \mathrm{~Hz}), 44.3(\mathrm{~d}, J=4.0 \mathrm{~Hz}), 28.8,16.6 .{ }^{31} \mathbf{P} \mathbf{N M R}\left(162 \mathrm{MHz}, \mathrm{CDCl}_{3}\right) \delta$ 30.37 (s). HRMS (ESI): ([M+Na] $]^{+}$) Calcd for $\mathrm{C}_{27} \mathrm{H}_{27} \mathrm{NaO}_{3} \mathrm{PS}: 485.1316$, Found: 485.1310. IR (film) $v 2954,2923,1971,1443,1362,1192,972,728,694 \mathrm{~cm}^{-1}$.

\section{4-(3-(2,6-dimethylphenoxy)prop-1-yn-1-yl)tetrahydro-2H-thiopyran-4-yl diphenylphosphinate (2e)}

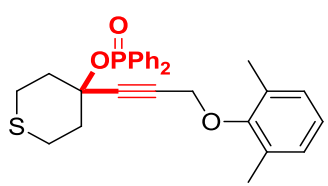

Yellow oil, (36.2 mg, $38 \%$ yield). ${ }^{1} \mathbf{H}$ NMR (400 MHz, $\left.\mathrm{CDCl}_{3}\right) \delta 7.85-7.75(\mathrm{~m}, 4 \mathrm{H}), 7.50-7.38$ (m, 6H), $7.05-6.90(\mathrm{~m}, 3 \mathrm{H}), 4.15(\mathrm{~s}, 2 \mathrm{H}), 2.81-2.67(\mathrm{~m}, 4 \mathrm{H}), 2.47-2.36(\mathrm{~m}, 4 \mathrm{H}), 2.21(\mathrm{~s}, 6 \mathrm{H})$. ${ }^{13} \mathbf{C}$ NMR $\left(101 \mathrm{MHz}, \mathrm{CDCl}_{3}\right) \delta$ 155.0, 134.2, 132.8, 131.8, 131.8, 131.5, 131.4, 131.1, 128.8, 128.4, 128.3, 124.4, 86.5, 85.3 (d, $J=4.0 \mathrm{~Hz}), 76.3$ (d, $J=9.1 \mathrm{~Hz}), 59.4,40.5$ (d, $J=4.0 \mathrm{~Hz}), 25.2,16.6$. ${ }^{31}$ P NMR (162 MHz, $\left.\mathrm{CDCl}_{3}\right) \delta 28.78$ (s). HRMS (ESI): $\left([\mathrm{M}+\mathrm{Na}]^{+}\right)$Calcd for $\mathrm{C}_{28} \mathrm{H}_{29} \mathrm{NaO}_{3} \mathrm{PS}$ : 499.1473, Found: 499.1467. IR (film) v 2921, 2849, 1716, 1584, 1438, 1184, 1129, 969, 743, 693 $\mathrm{cm}^{-1}$. 
4-(3-(2,6-dimethylphenoxy)prop-1-yn-1-yl)tetrahydro-2H-pyran-4-yl diphenylphosphinate (2f)

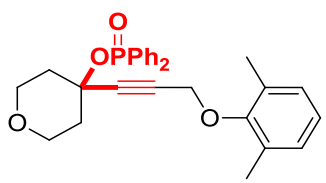

A white solid, m.p.: $107.4-108.0{ }^{\circ} \mathrm{C},(49.7 \mathrm{mg}, 54 \%$ yield $) .{ }^{1} \mathbf{H}$ NMR $\left(400 \mathrm{MHz}, \mathrm{CDCl}_{3}\right) \delta 7.87-$ $7.73(\mathrm{~m}, 4 \mathrm{H}), 7.52-7.37(\mathrm{~m}, 6 \mathrm{H}), 7.05-6.90(\mathrm{~m}, 3 \mathrm{H}), 4.16(\mathrm{~s}, 2 \mathrm{H}), 3.95-3.81(\mathrm{~m}, 2 \mathrm{H}), 3.69-$ $3.51(\mathrm{~m}, 2 \mathrm{H}), 2.31-2.24(\mathrm{~m} 2 \mathrm{H}), 2.21(\mathrm{~s}, 6 \mathrm{H}), 2.19-2.04(\mathrm{~m}, 2 \mathrm{H}) .{ }^{13} \mathbf{C}$ NMR $\left(101 \mathrm{MHz}, \mathrm{CDCl}_{3}\right)$ $\delta 154.9,134.1,132.7,131.8,131.8,131.5,131.4,131.1,128.8,128.4,128.2,124.4,85.6,85.4(\mathrm{~d}, J$ $=4.0 \mathrm{~Hz}), 74.5(\mathrm{~d}, J=8.1 \mathrm{~Hz}), 64.3,59.4,39.9(\mathrm{~d}, J=4.0 \mathrm{~Hz}), 16.6 .{ }^{31} \mathbf{P}$ NMR $\left(162 \mathrm{MHz}, \mathrm{CDCl}_{3}\right)$ $\delta 29.04$ (s). HRMS (ESI): $\left([\mathrm{M}+\mathrm{Na}]^{+}\right)$Calcd for $\mathrm{C}_{28} \mathrm{H}_{29} \mathrm{NaO}_{4} \mathrm{P}: 483.1701$, Found: 483.1695. IR (film) $v$ 2954, 2923, 2158, 1590, 1443, 1312, 1192, 972, 727, $694 \mathrm{~cm}^{-1}$.

\section{5-(2,6-dimethylphenoxy)-2-methylpent-3-yn-2-yl diphenylphosphinate (2g)}<smiles>Cc1cccc(C)c1OCC#CC(C)(C)O</smiles>

A white solid, m.p.: $109.4-110.2{ }^{\circ} \mathrm{C},(76.9 \mathrm{mg}, 92 \%$ yield $) .{ }^{1} \mathbf{H}$ NMR $\left(400 \mathrm{MHz}, \mathrm{CDCl}_{3}\right) \delta 7.88-$ $7.75(\mathrm{~m}, 4 \mathrm{H}), 7.54-7.35(\mathrm{~m}, 6 \mathrm{H}), 7.04-6.92(\mathrm{~m}, 3 \mathrm{H}), 4.16(\mathrm{~s}, 2 \mathrm{H}), 2.24(\mathrm{~s}, 6 \mathrm{H}), 1.81(\mathrm{~s}, 6 \mathrm{H}) .{ }^{13} \mathrm{C}$ NMR $\left(101 \mathrm{MHz}, \mathrm{CDCl}_{3}\right) \delta 155.1,134.4,133.0,131.7,131.6,131.6,131.5,131.2,128.8,128.3$, $128.2,124.3,87.7(\mathrm{~d}, J=5.0 \mathrm{~Hz}), 82.5,74.6(\mathrm{~d}, J=8.1 \mathrm{~Hz}), 59.6,31.7(\mathrm{~d}, J=3.0 \mathrm{~Hz}), 16.6 .{ }^{31} \mathbf{P}$ NMR $\left(162 \mathrm{MHz}, \mathrm{CDCl}_{3}\right) \delta 27.71$ (s). HRMS (ESI): $\left([\mathrm{M}+\mathrm{Na}]^{+}\right)$Calcd for $\mathrm{C}_{26} \mathrm{H}_{27} \mathrm{NaO}_{3} \mathrm{P}: 441.1596$, Found: 441.1590. IR (film) v 2923, 2854, 2161, 1590, 1439, 1375, 1224, 1194, 1109, 958, 744, 689 $\mathrm{cm}^{-1}$.

1-(2,6-dimethylphenoxy)-4-methylhept-2-yn-4-yl diphenylphosphinate (2h)

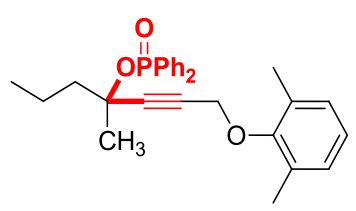

Pale yellow oil, (78.5 mg, $88 \%$ yield). ${ }^{1} \mathbf{H}$ NMR $\left(400 \mathrm{MHz}, \mathrm{CDCl}_{3}\right) \delta 7.90-7.73$ (m, 4H), $7.52-$ $7.32(\mathrm{~m}, 6 \mathrm{H}), 7.10-6.90(\mathrm{~m}, 3 \mathrm{H}), 4.28-4.18(\mathrm{~m}, 2 \mathrm{H}), 2.23(\mathrm{~s}, 6 \mathrm{H}), 2.08-1.98(\mathrm{~m}, 1 \mathrm{H}), 1.92-$ $1.82(\mathrm{~m}, 1 \mathrm{H}), 1.79(\mathrm{~s}, 3 \mathrm{H}), 1.64-1.43(\mathrm{~m}, 2 \mathrm{H}), 0.96(\mathrm{t}, J=8.0,3 \mathrm{H}) .{ }^{13} \mathbf{C} \mathbf{N M R}\left(101 \mathrm{MHz}, \mathrm{CDCl}_{3}\right)$ 
$\delta 155.1,134.5(\mathrm{~d}, J=34.3 \mathrm{~Hz}), 133.2,(\mathrm{~d}, J=30.3 \mathrm{~Hz}), 131.7(\mathrm{~d}, J=10.1 \mathrm{~Hz}), 131.6(\mathrm{~d}, J=6.1$ Hz), $131.4(\mathrm{~d}, J=24.2 \mathrm{~Hz}), 131.2(\mathrm{~d}, J=7.1 \mathrm{~Hz}), 128.7,128.3,128.2(\mathrm{~d}, J=2.1 \mathrm{~Hz}), 128.1,124.2$, $86.9(\mathrm{~d}, J=4.0 \mathrm{~Hz}), 83.5,77.8(\mathrm{~d}, J=9.1 \mathrm{~Hz}), 59.6,46.2(\mathrm{~d}, J=5.1 \mathrm{~Hz}), 29.5(\mathrm{~d}, J=3.0 \mathrm{~Hz}), 17.9$, 16.6, 14.0. ${ }^{31} \mathbf{P}$ NMR $\left(162 \mathrm{MHz}, \mathrm{CDCl}_{3}\right) \delta 27.42$ (s). HRMS (ESI): $\left([\mathrm{M}+\mathrm{Na}]^{+}\right)$Calcd for $\mathrm{C}_{28} \mathrm{H}_{31} \mathrm{NaO}_{3} \mathrm{P}: 469.1909$, Found: 469.1903. IR (film) v 2970, 2934, 2160, 1477, 1439, 1223, 1197, $1122,934,737,690 \mathrm{~cm}^{-1}$

\section{1-(2,6-dimethylphenoxy)-4-methyldec-2-yn-4-yl diphenylphosphinate (2i)}

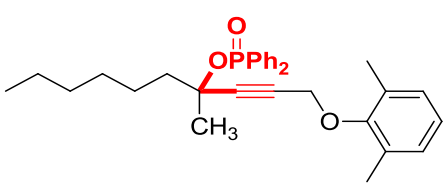

Colorless oil, (56.6 mg, $58 \%$ yield). ${ }^{1} \mathbf{H}$ NMR $\left(400 \mathrm{MHz}, \mathrm{CDCl}_{3}\right) \delta 7.86-7.75(\mathrm{~m}, 4 \mathrm{H}), 7.51-$ $7.37(\mathrm{~m}, 6 \mathrm{H}), 7.06-6.88(\mathrm{~m}, 3 \mathrm{H}), 4.25-4.12(\mathrm{~m}, 2 \mathrm{H}), 2.23(\mathrm{~s}, 6 \mathrm{H}), 2.08-1.99(\mathrm{~m}, 1 \mathrm{H}), 1.94-$ $1.85(\mathrm{~m}, 1 \mathrm{H}), 1.79(\mathrm{~s}, 3 \mathrm{H}), 1.55-1.42(\mathrm{~m}, 2 \mathrm{H}), 1.35-1.27(\mathrm{~m}, 6 \mathrm{H}), 0.95-0.88(\mathrm{~m}, 3 \mathrm{H}) .{ }^{13} \mathbf{C}$ NMR $\left(101 \mathrm{MHz}, \mathrm{CDCl}_{3}\right) \delta 155.2,134.5(\mathrm{~d}, J=34.3 \mathrm{~Hz}), 133.1(\mathrm{~d}, J=30.3 \mathrm{~Hz}), 131.7(\mathrm{~d}, J=10.1 \mathrm{~Hz})$, $131.6(\mathrm{~d}, J=6.1 \mathrm{~Hz}), 131.4(\mathrm{~d}, J=24.2 \mathrm{~Hz}), 131.2(\mathrm{~d}, J=8.1 \mathrm{~Hz}), 128.7,128.3,128.2(\mathrm{~d}, J=2.0$ Hz), 128.1, 124.2, 86.9 (d, $J=4.0 \mathrm{~Hz}), 83.5,77.9$ (d, $J=9.1 \mathrm{~Hz}), 59.7,44.1(\mathrm{~d}, J=5.1 \mathrm{~Hz}), 31.7$, $29.5(\mathrm{~d}, J=3.0 \mathrm{~Hz}), 29.2,24.5,22.6,16.6,14.1 .{ }^{31} \mathbf{P}$ NMR $\left(162 \mathrm{MHz}, \mathrm{CDCl}_{3}\right) \delta 27.44$ (s). HRMS (ESI): $\left([\mathrm{M}+\mathrm{Na}]^{+}\right)$Calcd for $\mathrm{C}_{31} \mathrm{H}_{37} \mathrm{NaO}_{3} \mathrm{P}: 511.2378$, Found: 511.2372. IR (film) v 2926, 2857, $2157,1973,1438,1227,1189,950,753,693 \mathrm{~cm}^{-1}$.

\section{6-(2,6-dimethylphenoxy)-2,3-dimethylhex-4-yn-3-yl diphenylphosphinate (2j)}

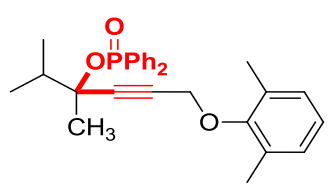

Yellow oil, (65.2 mg, $73 \%$ yield). ${ }^{1} \mathbf{H}$ NMR (400 MHz, $\left.\mathrm{CDCl}_{3}\right) \delta 7.90-7.75(\mathrm{~m}, 4 \mathrm{H}), 7.52-7.32$ (m, 6H), $7.10-6.85$ (m, 3H), 4.18 (q, J=15.5 Hz, 2H), 2.22 (s, 6H), $2.21-2.13(\mathrm{~m}, 1 \mathrm{H}), 1.77$ (s, 3H), $1.06(\mathrm{dd}, J=12.9,6.8 \mathrm{~Hz}, 6 \mathrm{H}) .{ }^{13} \mathbf{C} \mathbf{~ N M R}\left(101 \mathrm{MHz}, \mathrm{CDCl}_{3}\right) \delta 155.2,134.6(\mathrm{~d}, J=55.6 \mathrm{~Hz})$, $133.2(\mathrm{~d}, J=49.5 \mathrm{~Hz}), 131.9(\mathrm{~d}, J=10.1 \mathrm{~Hz}), 131.6(\mathrm{~d}, J=3.0 \mathrm{~Hz}), 131.5(\mathrm{~d}, J=3.0 \mathrm{~Hz}), 131.2$, $131.1(\mathrm{~d}, J=3.0 \mathrm{~Hz}), 128.7,128.3(\mathrm{~d}, J=13.1 \mathrm{~Hz}), 128.1(\mathrm{~d}, J=13.1 \mathrm{~Hz}), 124.2,86.1(\mathrm{~d}, J=4.0$ Hz), 84.2, $81.3(\mathrm{~d}, J=9.1 \mathrm{~Hz}), 59.6,39.7(\mathrm{~d}, J=6.1 \mathrm{~Hz}), 26.4(\mathrm{~d}, J=2.0 \mathrm{~Hz}), 17.8,17.5,16.6 .{ }^{31} \mathbf{P}$ 
NMR $\left(162 \mathrm{MHz}, \mathrm{CDCl}_{3}\right) \delta 27.56$ (s). HRMS (ESI): $\left([\mathrm{M}+\mathrm{Na}]^{+}\right)$Calcd for $\mathrm{C}_{28} \mathrm{H}_{31} \mathrm{NaO}_{3} \mathrm{P}: 469.1909$, Found: 469.1903. IR (film) v 2964, 2928, 2158, 1980, 1439, 1374, 1226, 1196, 1123, 947, 757, 695 $\mathrm{cm}^{-1}$.

2-cyclohexyl-5-(2,6-dimethylphenoxy)pent-3-yn-2-yl diphenylphosphinate (2k)

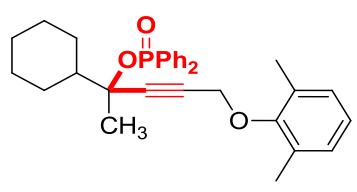

Yellow oil, (74.8 mg, $77 \%$ yield). ${ }^{1} \mathbf{H}$ NMR $\left(400 \mathrm{MHz}, \mathrm{CDCl}_{3}\right) \delta 7.91-7.71(\mathrm{~m}, 4 \mathrm{H}), 7.52-7.35$ (m, 6H), $7.05-6.85(\mathrm{~m}, 3 \mathrm{H}), 4.18$ (q, $J=15.5 \mathrm{~Hz}, 2 \mathrm{H}), 2.22(\mathrm{~s}, 6 \mathrm{H}), 2.08-1.98(\mathrm{~m}, 1 \mathrm{H}), 1.90(\mathrm{~d}$, $J=12.3 \mathrm{~Hz}, 1 \mathrm{H}), 1.85-1.78(\mathrm{~m}, 2 \mathrm{H}), 1.77(\mathrm{~s}, 3 \mathrm{H}), 1.69(\mathrm{~d}, J=12.2 \mathrm{~Hz}, 1 \mathrm{H}), 1.27-1.19(\mathrm{~m}, 3 \mathrm{H})$, $1.18-1.05(\mathrm{~m}, 3 \mathrm{H}) .{ }^{13} \mathbf{C} \mathbf{N M R}\left(101 \mathrm{MHz}, \mathrm{CDCl}_{3}\right) \delta 155.1,134.6(\mathrm{~d}, J=56.6 \mathrm{~Hz}), 133.3(\mathrm{~d}, J=$ $50.5 \mathrm{~Hz}), 131.9(\mathrm{~d}, J=10.1 \mathrm{~Hz}), 131.5(\mathrm{~d}, J=3.0 \mathrm{~Hz}), 131.5(\mathrm{~d}, J=2.0 \mathrm{~Hz}), 131.2(\mathrm{~d}, J=5.1 \mathrm{~Hz})$, 131.1, 128.7, 128.3 (d, $J=14.1 \mathrm{~Hz}), 128.1$ (d, $J=13.1 \mathrm{~Hz}), 124.2,86.4$ (d, $J=4.0 \mathrm{~Hz}), 84.3,80.9$ $(\mathrm{d}, J=9.4 \mathrm{~Hz}), 59.6,49.2(\mathrm{~d}, J=5.1 \mathrm{~Hz}), 27.5,27.3,26.7(\mathrm{~d}, J=2.0 \mathrm{~Hz}), 26.2,16.7 .{ }^{31} \mathbf{P}$ NMR $(162$ MHz, $\left.\mathrm{CDCl}_{3}\right) \delta 27.40$ (s). HRMS (ESI): ([M+Na] $]^{+}$) Calcd for $\mathrm{C}_{31} \mathrm{H}_{35} \mathrm{NaO}_{3} \mathrm{P}: 509.2222$, Found: 509.2216. IR (film) v 2925, 2853, 2166, 1588, 1437, 1183, 952, 752, 725, 690 $\mathrm{cm}^{-1}$.

6-(2,6-dimethylphenoxy)-2,2,3-trimethylhex-4-yn-3-yl diphenylphosphinate (2l)

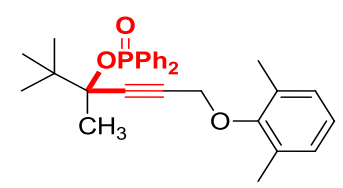

A white solid, m.p.: $118.3-119.5^{\circ} \mathrm{C},(67.2 \mathrm{mg}, 73 \%$ yield $) .{ }^{1} \mathbf{H} \mathbf{N M R}\left(400 \mathrm{MHz}, \mathrm{CDCl}_{3}\right) \delta 7.94-$ $7.70(\mathrm{~m}, 4 \mathrm{H}), 7.54-7.33(\mathrm{~m}, 6 \mathrm{H}), 7.10-6.85(\mathrm{~m}, 3 \mathrm{H}), 4.27-4.05(\mathrm{~m}, 2 \mathrm{H}), 2.22(\mathrm{~s}, 6 \mathrm{H}), 1.83(\mathrm{~s}$, 3H), $1.10(\mathrm{~s}, 9 \mathrm{H}) .{ }^{13} \mathbf{C}$ NMR $\left(101 \mathrm{MHz}, \mathrm{CDCl}_{3}\right) \delta 155.2,134.6(\mathrm{~d}, J=91.9 \mathrm{~Hz}), 133.2(\mathrm{~d}, J=82.8$ $\mathrm{Hz}), 132.1(\mathrm{~d}, J=11.1 \mathrm{~Hz}), 131.6(\mathrm{~d}, J=2.0 \mathrm{~Hz}), 131.5(\mathrm{~d}, J=3.0 \mathrm{~Hz}), 131.1(\mathrm{~d}, J=2.0 \mathrm{~Hz}), 131.0$, 128.7, 128.3 (d, $J=14.1 \mathrm{~Hz}), 128.0(\mathrm{~d}, J=13.1 \mathrm{~Hz}), 124.2,86.3(\mathrm{~d}, J=3.0 \mathrm{~Hz}), 84.3,83.7$ (d, $J=$ $10.1 \mathrm{~Hz}), 59.6,39.8(\mathrm{~d}, J=6.1 \mathrm{~Hz}), 25.3,24.8,16.7 .{ }^{31} \mathbf{P}$ NMR $\left(162 \mathrm{MHz}, \mathrm{CDCl}_{3}\right) \delta 27.38(\mathrm{~s})$. HRMS (ESI): ([M+Na] $]^{+}$) Calcd for $\mathrm{C}_{29} \mathrm{H}_{33} \mathrm{NaO}_{3} \mathrm{P}: 483.2065$, Found: 483.2059. IR (film) $v 2968$, $2922,2159,1976,1477,1436,1231,1187,1127,949,930,726,692 \mathrm{~cm}^{-1}$. 
5-(2,6-dimethylphenoxy)-1-(4-methoxyphenyl)-2-methylpent-3-yn-2-yl diphenylphosphinate (2m)

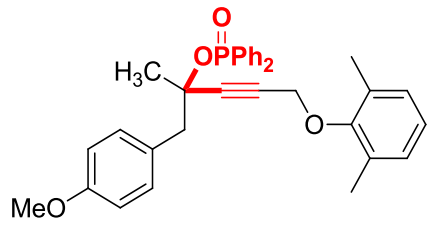

Yellow oil, (51.4 mg, $49 \%$ yield). ${ }^{1} \mathbf{H}$ NMR (400 MHz, $\left.\mathrm{CDCl}_{3}\right) \delta 7.76-7.68(\mathrm{~m}, 2 \mathrm{H}), 7.55-7.48$ (m, 2H), $7.46-7.34(\mathrm{~m}, 4 \mathrm{H}), 7.29-7.17(\mathrm{~m}, 4 \mathrm{H}), 7.01-6.90(\mathrm{~m}, 3 \mathrm{H}), 6.81(\mathrm{~d}, J=8.0 \mathrm{~Hz}, 2 \mathrm{H})$, $4.04(\mathrm{dd}, J=39.5,15.3 \mathrm{~Hz}, 2 \mathrm{H}), 3.80(\mathrm{~s}, 3 \mathrm{H}), 3.17(\mathrm{dd}, J=29.0,13.6 \mathrm{~Hz}, 2 \mathrm{H}), 2.19(\mathrm{~s}, 6 \mathrm{H}), 1.79$ (s, 3H). ${ }^{13} \mathbf{C}$ NMR $\left(101 \mathrm{MHz}, \mathrm{CDCl}_{3}\right) \delta 158.8,155.1,134.3(\mathrm{~d}, J=57.6 \mathrm{~Hz}), 132.9(\mathrm{~d}, J=53.5 \mathrm{~Hz})$, $132.2(\mathrm{~d}, J=1.0 \mathrm{~Hz}), 131.8(\mathrm{~d}, J=2.0 \mathrm{~Hz}), 131.6(\mathrm{~d}, J=2.0 \mathrm{~Hz}), 131.5,131.3(\mathrm{~d}, J=2.0 \mathrm{~Hz}), 131.2$ $(\mathrm{d}, J=2.0 \mathrm{~Hz}), 128.8(\mathrm{~d}, J=2.0 \mathrm{~Hz}), 128.3(\mathrm{~d}, J=14.1 \mathrm{~Hz}, 2.0 \mathrm{~Hz}), 128.0(\mathrm{~d}, J=14.1 \mathrm{~Hz}), 127.7$ $(\mathrm{d}, J=3.0 \mathrm{~Hz}), 124.4(\mathrm{~d}, J=2.0 \mathrm{~Hz}), 113.3,86.4,85.2(\mathrm{~d}, J=2.0 \mathrm{~Hz}), 59.5,55.3,48.8(\mathrm{~d}, J=3.0$ Hz), 29.6, 16.6. ${ }^{31} \mathbf{P}$ NMR (162 MHz, $\left.\mathrm{CDCl}_{3}\right) \delta 28.35$ (s). HRMS (ESI): $\left([\mathrm{M}+\mathrm{Na}]^{+}\right)$Calcd for $\mathrm{C}_{33} \mathrm{H}_{33} \mathrm{NaO}_{4} \mathrm{P}:$ 547.2014, Found: 547.2008. IR (film) v 2923, 2752, 2151, 1512, 1438, 1226, 1193 , $964,756,693 \mathrm{~cm}^{-1}$.

6-(2,6-dimethylphenoxy)-3-ethylhex-4-yn-3-yl diphenylphosphinate (2p)

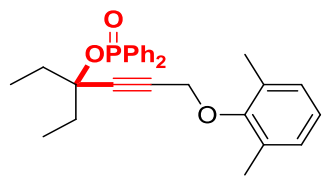

A white solid, m.p.: $55.5-56.2{ }^{\circ} \mathrm{C},(74.9 \mathrm{mg}, 84 \%$ yield $) .{ }^{1} \mathbf{H}$ NMR $\left(400 \mathrm{MHz}, \mathrm{CDCl}_{3}\right) \delta 7.83-$ $7.74(\mathrm{~m}, 4 \mathrm{H}), 7.46-7.33(\mathrm{~m}, 6 \mathrm{H}), 7.00-6.86(\mathrm{~m}, 3 \mathrm{H}), 4.14(\mathrm{~s}, 2 \mathrm{H}), 2.19(\mathrm{~s}, 6 \mathrm{H}), 2.10-1.97(\mathrm{~m}$, 4H), $0.99(\mathrm{t}, \mathrm{J}=7.4 \mathrm{~Hz}, 6 \mathrm{H}) .{ }^{13} \mathbf{C}$ NMR $\left(101 \mathrm{MHz}, \mathrm{CDCl}_{3}\right) \delta 155.2,134.6,133.2,131.5(\mathrm{~d}, \mathrm{~J}=10.1$ Hz), $131.3(\mathrm{~d}, \mathrm{~J}=40.4 \mathrm{~Hz}), 128.7,128.2$ (d, J = 13.1 Hz), 124.2, 85.9 (d, J = 5.1 Hz), 84.7, 82.1 (d, $\mathrm{J}=9.1 \mathrm{~Hz}), 59.6,33.5(\mathrm{~d}, \mathrm{~J}=3.7 \mathrm{~Hz}), 16.6,8.7 .{ }^{31} \mathbf{P}$ NMR $(162 \mathrm{MHz}, \mathrm{CDCl} 3) \delta 27.09(\mathrm{~s}) . \mathbf{H R M S}$ (ESI): $\left([\mathrm{M}+\mathrm{Na}]^{+}\right)$Calcd for $\mathrm{C}_{28} \mathrm{H}_{31} \mathrm{NaO}_{3} \mathrm{P}: 469.1909$, Found: 469.1903. IR (film) v 2967, 2157, $1728,1590,1437,1231,1187,1126,949,767,691 \mathrm{~cm}^{-1}$.

2-benzyl-5-(2,6-dimethylphenoxy)-1-phenylpent-3-yn-2-yl diphenylphosphinate (2q)<smiles>Cc1cccc(C)c1OCC#CC(Cc1ccccc1)(O[O+])[P+]([O-])(O)O</smiles> 
A white solid, m.p.: $112.6-113.7{ }^{\circ} \mathrm{C},(74.9 \mathrm{mg}, 84 \%$ yield $) .{ }^{1} \mathbf{H} \mathbf{N M R}\left(400 \mathrm{MHz}, \mathrm{CDCl}_{3}\right) \delta 7.43-$ $7.35(\mathrm{~m}, 8 \mathrm{H}), 7.35-7.29(\mathrm{~m}, 8 \mathrm{H}), 7.27-7.22(\mathrm{~m}, 4 \mathrm{H}), 7.06-6.96(\mathrm{~m}, 3 \mathrm{H}), 3.92(\mathrm{~s}, 2 \mathrm{H}), 3.44(\mathrm{~d}$, $J=13.5 \mathrm{~Hz}, 2 \mathrm{H}), 3.31(\mathrm{~d}, J=13.5 \mathrm{~Hz}, 2 \mathrm{H}), 2.21(\mathrm{~s}, 6 \mathrm{H}) .{ }^{13} \mathrm{C} \mathbf{N M R}\left(101 \mathrm{MHz}, \mathrm{CDCl}_{3}\right) \delta 155.0$, 135.5, 134.2, 132.8, 131.5, 131.4, 131.3, 131.2, 131.1, 128.8, 128.0, 127.9, 127.0, 124.4, 88.3, 84.8 $(\mathrm{d}, J=4.0 \mathrm{~Hz}), 79.2(\mathrm{~d}, J=9.1 \mathrm{~Hz}), 59.1,47.7(\mathrm{~d}, J=3.0 \mathrm{~Hz}), 16.5 .{ }^{31} \mathbf{P} \mathbf{N M R}\left(162 \mathrm{MHz}, \mathrm{CDCl}_{3}\right)$ $\delta 28.87$ (s). HRMS (ESI): ([M+Na $\left.]^{+}\right)$Calcd for $\mathrm{C}_{38} \mathrm{H}_{35} \mathrm{NaO}_{3} \mathrm{P}: 593.2222$, Found: 593.2216. IR (film) v 2915, 2849, 2158, 1730, 1470, 1248, 1162, 961, 729, $691 \mathrm{~cm}^{-1}$.

\section{6-(2,6-dimethylphenoxy)hex-4-yn-3-yl diphenylphosphinate (2r)}

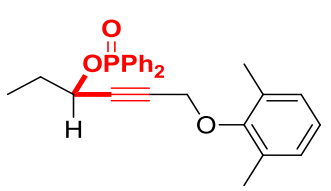

Yellow oil, (49.3 mg, $59 \%$ yield). ${ }^{1} \mathbf{H}$ NMR (400 MHz, $\left.\mathrm{CDCl}_{3}\right) \delta 7.84-7.60(\mathrm{~m}, 4 \mathrm{H}), 7.50-7.23$ (m, 6H), $7.01-6.73(\mathrm{~m}, 3 \mathrm{H}), 5.10-4.90(\mathrm{~m}, 1 \mathrm{H}), 4.31(\mathrm{~s}, 2 \mathrm{H}), 2.20(\mathrm{~s}, 6 \mathrm{H}), 1.90-1.77(\mathrm{~m}, 2 \mathrm{H})$, $0.95(\mathrm{t}, J=7.3 \mathrm{~Hz}, 3 \mathrm{H}) .{ }^{13} \mathbf{C}$ NMR $\left(101 \mathrm{MHz}, \mathrm{CDCl}_{3}\right) \delta 155.2,132.3(\mathrm{~d}, J=77.8 \mathrm{~Hz}), 132.2,132.2$ $(\mathrm{d}, J=4.0 \mathrm{~Hz}), 132.1(\mathrm{~d}, J=5.1 \mathrm{~Hz}), 131.4,131.3,131.1,131.0(\mathrm{~d}, J=69.7 \mathrm{~Hz}), 128.6(\mathrm{~d}, J=41.4$ $\mathrm{Hz}), 128.4(\mathrm{~d}, J=26.3 \mathrm{~Hz}), 124.4,84.3(\mathrm{~d}, J=5.0 \mathrm{~Hz}), 82.7,66.7(\mathrm{~d}, J=6.1 \mathrm{~Hz}), 59.8,30.2(\mathrm{~d}, J$ $=5.1 \mathrm{~Hz}), 16.6,9.2 .{ }^{31} \mathbf{P}$ NMR $\left(162 \mathrm{MHz}, \mathrm{CDCl}_{3}\right) \delta 32.37$ (s). HRMS (ESI): $\left([\mathrm{M}+\mathrm{Na}]^{+}\right)$Calcd for $\mathrm{C}_{26} \mathrm{H}_{27} \mathrm{NaO}_{3} \mathrm{P}: 441.1596$, Found: 441.1590. IR (film) v 2971, 2924, 1967, 1438, 1225, 1128, 965 , $726,693 \mathrm{~cm}^{-1}$.

1-cyclohexyl-4-(2,6-dimethylphenoxy)but-2-yn-1-yl diphenylphosphinate (2s)

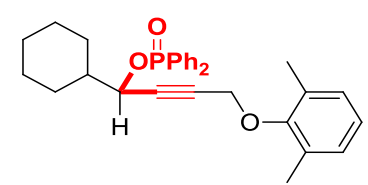

Pale yellow oil, (43.4 mg, $46 \%$ yield). ${ }^{1} \mathbf{H}$ NMR (400 MHz, $\left.\mathrm{CDCl}_{3}\right) \delta 7.90-7.77(\mathrm{~m}, 4 \mathrm{H}), 7.57-$ $7.38(\mathrm{~m}, 6 \mathrm{H}), 7.06-6.87(\mathrm{~m}, 3 \mathrm{H}), 5.01-4.89(\mathrm{~m}, 1 \mathrm{H}), 4.45-4.31(\mathrm{~m}, 2 \mathrm{H}), 2.28(\mathrm{~s}, 6 \mathrm{H}), 1.89(\mathrm{~d}$, $J=11.9 \mathrm{~Hz}, 1 \mathrm{H}), 1.78(\mathrm{t}, J=12.5 \mathrm{~Hz}, 4 \mathrm{H}), 1.67(\mathrm{~d}, J=11.6 \mathrm{~Hz}, 1 \mathrm{H}), 1.27-1.08(\mathrm{~m}, 5 \mathrm{H}) .{ }^{13} \mathrm{C}$ NMR $\left(101 \mathrm{MHz}, \mathrm{CDCl}_{3}\right) \delta 155.1,132.4(\mathrm{~d}, J=76.8 \mathrm{~Hz}), 132.3(\mathrm{~d}, J=10.1 \mathrm{~Hz}), 132.1,132.1$, $131.3(\mathrm{~d}, J=10.1 \mathrm{~Hz}), 131.2,131.1(\mathrm{~d}, J=67.7 \mathrm{~Hz}), 128.8,128.5(\mathrm{~d}, J=14.1 \mathrm{~Hz}), 128.2(\mathrm{~d}, J=$ $14.1 \mathrm{~Hz}), 124.3,83.5(\mathrm{~d}, J=4.0 \mathrm{~Hz}), 83.4,69.8(\mathrm{~d}, J=6.1 \mathrm{~Hz}), 59.7,43.4(\mathrm{~d}, J=4.0 \mathrm{~Hz}), 28.4$, 
27.7, 26.2, $25.7(\mathrm{~d}, J=6.1 \mathrm{~Hz}), 16.7 .{ }^{31} \mathbf{P}$ NMR $\left(162 \mathrm{MHz}, \mathrm{CDCl}_{3}\right) \delta 32.21$ (s). HRMS (ESI): $\left([\mathrm{M}+\mathrm{Na}]^{+}\right)$Calcd for $\mathrm{C}_{30} \mathrm{H}_{33} \mathrm{NaO}_{3} \mathrm{P}: 495.2065$, Found: 495.2059. IR (film) v 2917, 2849, 2158, 1937, $1225,1179,1113,734,695 \mathrm{~cm}^{-1}$.

1-(3-(2,6-dimethylphenoxy)prop-1-yn-1-yl)cyclohexyl bis(4-methoxyphenyl)phosphinate (2t)

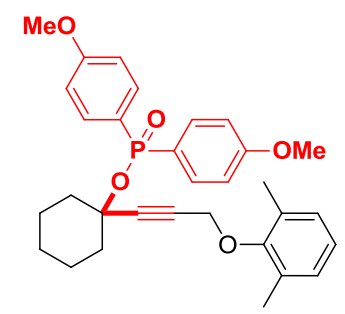

Yellow oil, (55.5 mg, $58 \%$ yield). ${ }^{1} \mathbf{H}$ NMR (400 MHz, $\left.\mathrm{CDCl}_{3}\right) \delta 7.77-7.67$ (m, 4H), 7.00 (d, $J=$ $8.0 \mathrm{~Hz}, 2 \mathrm{H}), 6.96-6.87$ (m, 5H), $4.24(\mathrm{~s}, 2 \mathrm{H}), 3.82(\mathrm{~s}, 6 \mathrm{H}), 2.23(\mathrm{~s}, 6 \mathrm{H}), 2.12-2.01(\mathrm{~m}, 4 \mathrm{H}), 1.73$ - $1.64(\mathrm{~m}, 2 \mathrm{H}), 1.56-1.45(\mathrm{~m}, 3 \mathrm{H}), 1.34-1.30(\mathrm{~m}, 1 \mathrm{H}) .{ }^{13} \mathbf{C}$ NMR $\left(101 \mathrm{MHz}, \mathrm{CDCl}_{3}\right) \delta 162.0(\mathrm{~d}$, $J=3.0 \mathrm{~Hz}), 155.2,133.4,133.2,131.2,128.7,126.5,125.0,124.2,113.7,113.6,86.8(\mathrm{~d}, J=4.0$ $\mathrm{Hz}), 84.2,59.8,55.3,39.8$ (d, $J=4.0 \mathrm{~Hz}), 24.8,22.9,16.6 .{ }^{31} \mathbf{P}$ NMR $\left(162 \mathrm{MHz}, \mathrm{CDCl}_{3}\right) \delta 28.43$ (s). HRMS (ESI): $\left([\mathrm{M}+\mathrm{Na}]^{+}\right)$Calcd for $\mathrm{C}_{31} \mathrm{H}_{35} \mathrm{NaO}_{5} \mathrm{P}: 541.2120$, Found: 541.2114. IR (film) $v$ $2926,2853,2158,1696,1503,1251,1210,1182,971,803,694 \mathrm{~cm}^{-1}$.

\section{5-(2,6-dimethylphenoxy)-2-methylpent-3-yn-2-yl bis(4-fluorophenyl)phosphinate (2u)}

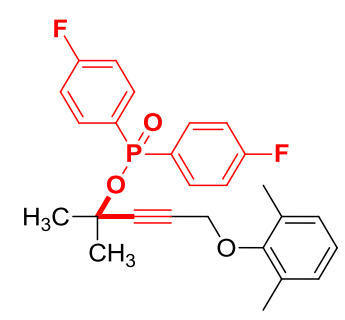

Yellow oil, (55.0 mg, $55 \%$ yield). ${ }^{1} \mathbf{H}$ NMR (400 MHz, $\left.\mathrm{CDCl}_{3}\right) \delta 7.85-7.70(\mathrm{~m}, 4 \mathrm{H}), 7.15-7.05$ (m, 4H), $7.04-6.90(\mathrm{~m}, 3 \mathrm{H}), 4.22(\mathrm{~s}, 2 \mathrm{H}), 2.24$ (s, 6H), 1.80 (s, 6H). ${ }^{13} \mathbf{C}$ NMR (101 MHz, $\left.\mathrm{CDCl}_{3}\right)$ $\delta 164.9(\mathrm{~d}, J=253.2 \mathrm{~Hz}), 155.0,133.9(\mathrm{dd}, J=11.6,8.8 \mathrm{~Hz}), 131.1,130.2(\mathrm{~d}, J=3.0 \mathrm{~Hz}), 128.8$, 124.3, $115.6(\mathrm{dd}, J=21.3,14.6 \mathrm{~Hz}), 87.5(\mathrm{~d}, J=4.0 \mathrm{~Hz}), 82.7,74.9(\mathrm{~d}, J=8.0 \mathrm{~Hz}), 59.6,31.7(\mathrm{~d}, J$ $=4.0 \mathrm{~Hz}), 16.5 .{ }^{31} \mathbf{P}$ NMR $\left(162 \mathrm{MHz}, \mathrm{CDCl}_{3}\right) \delta 26.07$ (s). HRMS (ESI): $\left([\mathrm{M}+\mathrm{Na}]^{+}\right)$Calcd for $\mathrm{C}_{26} \mathrm{H}_{25} \mathrm{~F}_{2} \mathrm{NaO}_{3} \mathrm{P}: 477.1407$, Found: 477.1401. IR (film) v 3050, 2974, 2158, 1976, 1591, 1497, 1374 , $1220,1191,968,945,827,781,696 \mathrm{~cm}^{-1}$. 


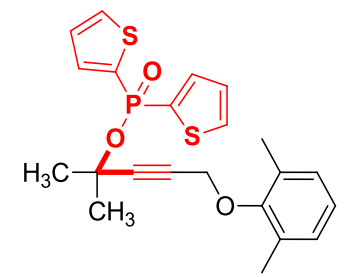

A pale yellow solid, m.p.: $135.5-136.9^{\circ} \mathrm{C},(39.6 \mathrm{mg}, 46 \%$ yield $) .{ }^{1} \mathbf{H} \mathbf{~ N M R}\left(400 \mathrm{MHz}, \mathrm{CDCl}_{3}\right) \delta$ $7.68-7.57(\mathrm{~m}, 4 \mathrm{H}), 7.15-7.08(\mathrm{~m}, 2 \mathrm{H}), 7.03-6.92(\mathrm{~m}, 3 \mathrm{H}), 4.30(\mathrm{~s}, 2 \mathrm{H}), 2.26(\mathrm{~s}, 6 \mathrm{H}), 1.80(\mathrm{~s}$, 6H). ${ }^{13} \mathbf{C}$ NMR (101 MHz, $\left.\mathrm{CDCl}_{3}\right) \delta 155.2,135.9,135.8,135.3,133.7,133.3,133.2,131.2,128.8$, 128.0, 127.9, 124.3, 87.1, 82.6, $75.6(\mathrm{~d}, J=9.1 \mathrm{~Hz}), 59.7,31.7(\mathrm{~d}, J=4.0 \mathrm{~Hz}), 16.6 .{ }^{31}$ P NMR $(162$ MHz, $\left.\mathrm{CDCl}_{3}\right) \delta 14.69$ (s). HRMS (ESI): ([M+Na $\left.]^{+}\right)$Calcd for $\mathrm{C}_{22} \mathrm{H}_{23} \mathrm{NaO}_{3} \mathrm{PS}_{2}: 453.0724$, Found: 453.0718. IR (film) v 3095, 2922, 2852, 2206, 1714, 1499, 1404, 1217, 1184, 1018, 948, 715, 661 $\mathrm{cm}^{-1}$.

\section{5-(2,6-dimethylphenoxy)-2-methylpent-3-yn-2-yl di(naphthalen-2-yl)phosphinate (2w)}

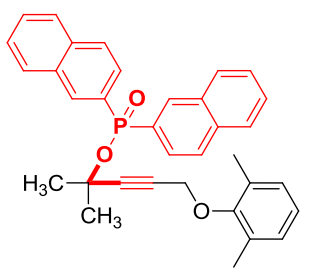

A white solid, m.p.: $62.5-62.9^{\circ} \mathrm{C},(77.6 \mathrm{mg}, 77 \%$ yield $) .{ }^{1} \mathbf{H}$ NMR $\left(400 \mathrm{MHz}, \mathrm{CDCl}_{3}\right) \delta 8.81-$ $8.67(\mathrm{~m}, 2 \mathrm{H}), 8.22-8.10(\mathrm{~m}, 2 \mathrm{H}), 7.99(\mathrm{~d}, J=8.2 \mathrm{~Hz}, 2 \mathrm{H}), 7.91-7.84(\mathrm{~m}, 2 \mathrm{H}), 7.53-7.45(\mathrm{~m}$, 6H), $7.00-6.91(\mathrm{~m}, 3 \mathrm{H}), 4.02(\mathrm{~s}, 2 \mathrm{H}), 2.15$ (s, 6H), 1.87 (s, 6H). ${ }^{13} \mathbf{C}$ NMR (101 MHz, $\left.\mathrm{CDCl}_{3}\right) \delta$ 155.1, 133.8, 133.7, 133.6, 133.2 (d, $J=3.0 \mathrm{~Hz}), 131.2,133.1$ (d, $J=10.1 \mathrm{~Hz}), 130.4,128.9$ (d, $J=28.3$ $\mathrm{Hz}), 128.8(\mathrm{~d}, J=1.0 \mathrm{~Hz}), 128.7,127.1,127.0(\mathrm{~d}, J=4.0 \mathrm{~Hz}), 126.2,124.6,124.5,124.2,87.6(\mathrm{~d}, J=$ $4.4 \mathrm{~Hz}), 75.3(\mathrm{~d}, J=8.1 \mathrm{~Hz}), 82.3,59.6,31.6(\mathrm{~d}, J=4.1 \mathrm{~Hz}), 16.5 .{ }^{31} \mathbf{P} \mathbf{N M R}\left(162 \mathrm{MHz}, \mathrm{CDCl}_{3}\right) \delta 30.75$ (s). HRMS (ESI): $\left([\mathrm{M}+\mathrm{Na}]^{+}\right)$Calcd for $\mathrm{C}_{34} \mathrm{H}_{31} \mathrm{NaO}_{3} \mathrm{P}: 541.1909$, Found: 541.1903. IR (film) $v$ $3048,2922,2331,1506,1261,1189,986,906,773,726,680,560 \mathrm{~cm}^{-1}$.

\section{2-methyl-5-phenoxypent-3-yn-2-yl diphenylphosphinate (2x)}

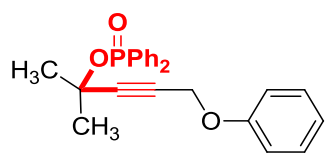


Pale yellow oil, (56.9 mg, $73 \%$ yield). ${ }^{1} \mathbf{H}$ NMR $\left(400 \mathrm{MHz}, \mathrm{CDCl}_{3}\right) \delta 7.91-7.78(\mathrm{~m}, 4 \mathrm{H}), 7.53-$ $7.39(\mathrm{~m}, 6 \mathrm{H}), 7.28(\mathrm{t}, J=8.0 \mathrm{~Hz}, 2 \mathrm{H}), 6.98(\mathrm{t}, J=7.3 \mathrm{~Hz}, 1 \mathrm{H}), 6.83(\mathrm{~d}, J=8.0 \mathrm{~Hz}, 2 \mathrm{H}), 4.35(\mathrm{~s}$, 2H), $1.79(\mathrm{~s}, 6 \mathrm{H}) .{ }^{13} \mathrm{C}$ NMR $\left(101 \mathrm{MHz}, \mathrm{CDCl}_{3}\right) \delta 157.6,134.3,133.0,131.8,131.7,131.6,131.5$, 129.4, 128.4, 128.2, 121.4, 114.9, $88.3(\mathrm{~d}, J=5.1 \mathrm{~Hz}), 81.6,74.5(\mathrm{~d}, J=8.1 \mathrm{~Hz}), 55.8,31.72(\mathrm{~d}, J$ $=4.0 \mathrm{~Hz}) .{ }^{31} \mathbf{P}$ NMR $\left(162 \mathrm{MHz}, \mathrm{CDCl}_{3}\right) \delta 28.00$ (s). HRMS (ESI): $\left([\mathrm{M}+\mathrm{Na}]^{+}\right)$Calcd for $\mathrm{C}_{24} \mathrm{H}_{23} \mathrm{NaO}_{3} \mathrm{P}: 413.1283$, Found: 413.1277. IR (film) v 2919, 2852, 2161, 1975, 1661, 1438, 1224 , $1128,1006,754,725,691 \mathrm{~cm}^{-1}$.

\section{2-methyl-5-(p-tolyloxy)pent-3-yn-2-yl diphenylphosphinate (2y)}

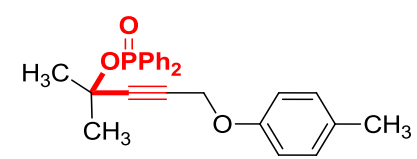

A white solid, m.p.: $66.2-67.0{ }^{\circ} \mathrm{C},(75.9 \mathrm{mg}, 94 \%$ yield $) .{ }^{1} \mathbf{H}$ NMR $\left(400 \mathrm{MHz}, \mathrm{CDCl}_{3}\right) \delta 7.94-$ $7.73(\mathrm{~m}, 4 \mathrm{H}), 7.54-7.35(\mathrm{~m}, 6 \mathrm{H}), 7.07(\mathrm{~d}, J=8.0 \mathrm{~Hz}, 2 \mathrm{H}), 6.73(\mathrm{~d}, J=8.0 \mathrm{~Hz}, 2 \mathrm{H}), 4.31(\mathrm{~s}, 2 \mathrm{H})$, $2.29(\mathrm{~s}, 3 \mathrm{H}), 1.80(\mathrm{~s}, 6 \mathrm{H}) .{ }^{13} \mathrm{C}$ NMR $\left(101 \mathrm{MHz}, \mathrm{CDCl}_{3}\right) \delta$ 155.6, 134.4, 133.0, 131.8, 131.7, 131.6, 131.5, 130.6, 129.8, 128.4, 128.2, 114.8, 88.2 (d, $J=5.1 \mathrm{~Hz}), 81.9,74.5(\mathrm{~d}, J=8.1 \mathrm{~Hz}), 55.9,31.7$ (d, $J=4.0 \mathrm{~Hz}), 20.1 .{ }^{31} \mathbf{P}$ NMR (162 MHz, CDCl 3 ) $\delta 27.92$ (s). HRMS (ESI): ([M+Na $]^{+}$) Calcd for $\mathrm{C}_{25} \mathrm{H}_{25} \mathrm{NaO}_{3} \mathrm{P}: 427.1439$, Found: 427.1433. IR (film) v 2972, 1966, 1586, 1507, 1439, 1213, 1108 , $954,725,692 \mathrm{~cm}^{-1}$.

\section{1-(prop-1-yn-1-yl)cyclohexyl diphenylphosphinate (2aa)}

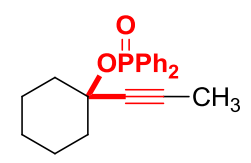

A white solid, m.p.: $95.6-97.0{ }^{\circ} \mathrm{C},\left(39.9 \mathrm{mg}, 59 \%\right.$ yield). ${ }^{1} \mathbf{H}$ NMR $\left(400 \mathrm{MHz}, \mathrm{CDCl}_{3}\right) \delta 7.90-$ $7.75(\mathrm{~m}, 4 \mathrm{H}), 7.52-7.32(\mathrm{~m}, 6 \mathrm{H}), 2.15-2.00(\mathrm{~m}, 4 \mathrm{H}), 1.76-1.66(\mathrm{~m}, 2 \mathrm{H}), 1.63-1.54(\mathrm{~m}, 2 \mathrm{H})$, $1.46(\mathrm{~s}, 1 \mathrm{H}), 1.42(\mathrm{~s}, 3 \mathrm{H}), 1.37-1.30(\mathrm{~m}, 1 \mathrm{H}) .{ }^{13} \mathbf{C} \mathbf{N M R}\left(101 \mathrm{MHz}, \mathrm{CDCl}_{3}\right) \delta 135.0,133.6,131.6$, 131.5, 131.4, 131.3, 128.2, 128.0, 85.9, 79.6 (d, $J=4.0 \mathrm{~Hz}), 78.5$ (d, $J=9.4 \mathrm{~Hz}), 40.3$ (d, $J=3.0$ Hz), 24.9, 23.0, 3.4. ${ }^{31}$ P NMR (162 MHz, $\left.\mathrm{CDCl}_{3}\right) \delta 27.13$ (s). HRMS (ESI): ([M+Na $]^{+}$) Calcd for $\mathrm{C}_{21} \mathrm{H}_{23} \mathrm{NaO}_{2} \mathrm{P}: 361.1333$, Found: 361.1327. IR (film) v 2953, 2860, 2158, 1558, 1480, 1171, 1069, $950,774,691 \mathrm{~cm}^{-1}$. 


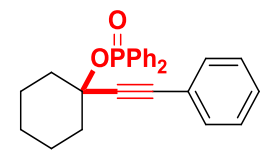

A white solid, m.p.: $109.0-109.4{ }^{\circ} \mathrm{C},(60.1 \mathrm{mg}, 75 \%$ yield $) .{ }^{1} \mathbf{H} \mathbf{N M R}\left(400 \mathrm{MHz}, \mathrm{CDCl}_{3}\right) \delta 7.94-$ $7.74(\mathrm{~m}, 4 \mathrm{H}), 7.51-7.31(\mathrm{~m}, 6 \mathrm{H}), 7.27-7.13(\mathrm{~m}, 3 \mathrm{H}), 6.99(\mathrm{~d}, J=7.4 \mathrm{~Hz}, 2 \mathrm{H}), 2.32-2.21(\mathrm{~m}$, 2H), $2.20-2.09(\mathrm{~m}, 2 \mathrm{H}), 1.84-1.72(\mathrm{~m}, 2 \mathrm{H}), 1.71-1.60(\mathrm{~m}, 2 \mathrm{H}), 1.57-1.48(\mathrm{~s}, 1 \mathrm{H}), 1.43-1.32$ (m, 1H). ${ }^{13} \mathbf{C}$ NMR $\left(101 \mathrm{MHz}, \mathrm{CDCl}_{3}\right) \delta 134.8,133.4,131.6,131.6,131.5,131.5,128.3,128.2$, 127.8, 122.2, 89.0, 78.5, 40.3 (d, $J=3.8 \mathrm{~Hz}), 24.9,23.1 .{ }^{31}$ P NMR $\left(162 \mathrm{MHz}, \mathrm{CDCl}_{3}\right) \delta 27.54(\mathrm{~s})$. HRMS (ESI): ([M+H] $]^{+}$Calcd for $\mathrm{C}_{26} \mathrm{H}_{26} \mathrm{O}_{2} \mathrm{P}: 401.1670$, Found: 401.1664. IR (film) $v 2933,2859$, $2158,1592,1437,1232,1113,981,751,690 \mathrm{~cm}^{-1}$.

\section{1-(phenylethynyl)cyclobutyl diphenylphosphinate (2ac)}

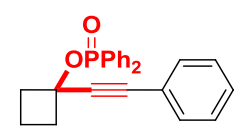

A white solid, m.p.: $91.8-92.7{ }^{\circ} \mathrm{C},(61.8 \mathrm{mg}, 83 \%$ yield $) .{ }^{1} \mathbf{H}$ NMR $\left(400 \mathrm{MHz}, \mathrm{CDCl}_{3}\right) \delta 7.93-$ $7.78(\mathrm{~m}, 4 \mathrm{H}), 7.52-7.35(\mathrm{~m}, 6 \mathrm{H}), 7.26-7.18(\mathrm{~m}, 3 \mathrm{H}), 7.13-7.04(\mathrm{~m}, 2 \mathrm{H}), 2.95-2.80(\mathrm{~m}, 2 \mathrm{H})$, $2.68-2.50(\mathrm{~m}, 2 \mathrm{H}), 1.98-1.89(\mathrm{~m}, 2 \mathrm{H}) .{ }^{13} \mathbf{C} \mathbf{N M R}\left(101 \mathrm{MHz}, \mathrm{CDCl}_{3}\right) \delta 133.7,132.4,131.8(\mathrm{~d}, J$ $=3.0 \mathrm{~Hz}), 131.7,131.6,128.4,128.1(\mathrm{~d}, J=27.2 \mathrm{~Hz}), 122.2,89.7(\mathrm{~d}, J=4.0 \mathrm{~Hz}), 86.6,73.9(\mathrm{~d}, J$ $=9.1 \mathrm{~Hz}), 39.3(\mathrm{~d}, J=5.0 \mathrm{~Hz}), 14.2 .{ }^{31} \mathbf{P}$ NMR $\left(162 \mathrm{MHz}, \mathrm{CDCl}_{3}\right) \delta 29.02$ (s). HRMS (ESI): $\left([\mathrm{M}+\mathrm{H}]^{+}\right)$Calcd for $\mathrm{C}_{24} \mathrm{H}_{22} \mathrm{O} 2 \mathrm{P}: 373.1357$, Found: 373.1351. IR (film) v 3050, 2999, 2237, 1591, $1444,1230,1124,1080,948,865,755,726,690 \mathrm{~cm}^{-1}$.

\section{1-(phenylethynyl)cyclopropyl diphenylphosphinate (2ad)}

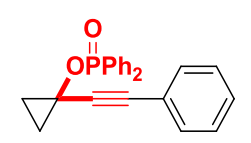

A white solid, m.p.: $80.7-81.2{ }^{\circ} \mathrm{C}\left(61.8 \mathrm{mg}, 52 \%\right.$ yield). ${ }^{1} \mathbf{H}$ NMR $\left(400 \mathrm{MHz}, \mathrm{CDCl}_{3}\right) \delta 7.96-$ $7.80(\mathrm{~m}, 4 \mathrm{H}), 7.54-7.37(\mathrm{~m}, 6 \mathrm{H}), 7.26-7.16(\mathrm{~m}, 3 \mathrm{H}), 7.10-7.03(\mathrm{~m}, 2 \mathrm{H}), 1.68(\mathrm{q}, J=5.7 \mathrm{~Hz}$, 2H), $1.20(\mathrm{dd}, J=8.4,5.5 \mathrm{~Hz}, 2 \mathrm{H}) .{ }^{13} \mathbf{C}$ NMR $\left(101 \mathrm{MHz}, \mathrm{CDCl}_{3}\right) \delta 132.5,132.2,132.1,131.9$, 131.6, 131.8, 131.2, 128.4, 128.3, 128.3, 128.0, 122.2, 88.2, 84.8, 50.7 (d, $J=8.0 \mathrm{~Hz}), 17.1$ (d, $J=$ $4.0 \mathrm{~Hz}) .{ }^{31} \mathbf{P}$ NMR $\left(162 \mathrm{MHz}, \mathrm{CDCl}_{3}\right) \delta 32.31$ (s). HRMS (ESI): $\left([\mathrm{M}+\mathrm{H}]^{+}\right)$Calcd for $\mathrm{C}_{23} \mathrm{H}_{20} \mathrm{O}_{2} \mathrm{P}$ : 
359.1201, Found: 359.1195. IR (film) v 3048, 2019, 2226, 1999, 1438, 1239, 1111, 927, 844, $691 \mathrm{~cm}^{-1}$.

2-methyl-4-phenylbut-3-yn-2-yl diphenylphosphinate (2ae)<smiles>CC(C)(O)O[Na]</smiles>

A pale yellow solid, m.p.: $148.6-149.1^{\circ} \mathrm{C},\left(38.2 \mathrm{mg}, 53 \%\right.$ yield). ${ }^{1} \mathbf{H} \mathbf{N M R}\left(400 \mathrm{MHz}, \mathrm{CDCl}_{3}\right) \delta$ $7.93-7.80$ (m, 4H), $7.49-7.38$ (m, 6H), $7.30-7.18(\mathrm{~m}, 3 \mathrm{H}), 7.05$ (d, J= 7.1 Hz, 2H), $1.92(\mathrm{~s}$, 6H). ${ }^{13} \mathbf{C}$ NMR $\left(101 \mathrm{MHz}, \mathrm{CDCl}_{3}\right) \delta 134.5,133.1,131.6,131.6,131.6,131.5,131.3(\mathrm{~d}, J=10.1$ Hz), 128.4, 128.3, 128.2, 127.9, 122.0 (d, $J=7.1 \mathrm{~Hz}), 90.2$ (d, $J=4.0 \mathrm{~Hz}), 86.6,75.2(\mathrm{~d}, J=9.1$ Hz), $32.1(\mathrm{~d}, J=4.0 \mathrm{~Hz}) \cdot{ }^{31} \mathbf{P}$ NMR $\left(162 \mathrm{MHz}, \mathrm{CDCl}_{3}\right) \delta 27.91$ (s). HRMS (ESI): ([M+Na $\left.]^{+}\right)$Calcd for $\mathrm{C}_{23} \mathrm{H}_{21} \mathrm{NaO}_{2} \mathrm{P}$ : 383.1177, Found: 383.1171. IR (film) $v$ 2918, 2850, 2157, 1681, 1437, 1177, $1120,954,726,690 \mathrm{~cm}^{-1}$.

3-methyl-1-phenylhept-6-en-1-yn-3-yl diphenylphosphinate (2af)

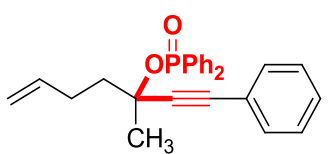

A white solid, m.p.: $161.2-162.0{ }^{\circ} \mathrm{C},(54.4 \mathrm{mg}, 68 \%$ yield $) .{ }^{1} \mathbf{H} \mathbf{N M R}\left(400 \mathrm{MHz}, \mathrm{CDCl}_{3}\right) \delta 7.96-$ $7.75(\mathrm{~m}, 4 \mathrm{H}), 7.47-7.33(\mathrm{~m}, 6 \mathrm{H}), 7.26-7.13(\mathrm{~m}, 3 \mathrm{H}), 7.06-6.96(\mathrm{~m}, 2 \mathrm{H}), 5.96-5.82(\mathrm{~m}, 1 \mathrm{H})$, $5.15-4.94(\mathrm{~m}, 2 \mathrm{H}), 2.52-2.33(\mathrm{~m}, 2 \mathrm{H}), 2.28-2.18(\mathrm{~m}, 1 \mathrm{H}), 2.12-2.02(\mathrm{~m}, 1 \mathrm{H}), 1.91(\mathrm{~s}, 3 \mathrm{H})$. ${ }^{13} \mathrm{C}$ NMR (101 MHz, $\left.\mathrm{CDCl}_{3}\right) \delta$ 137.8, $131.8(\mathrm{~d}, J=10.1 \mathrm{~Hz}), 131.7,131.6(\mathrm{~d}, J=3.0 \mathrm{~Hz}), 131.5$ (d, $J=2.0 \mathrm{~Hz}), 131.3(\mathrm{~d}, J=10.1 \mathrm{~Hz}), 128(\mathrm{~d}, J=6.1 \mathrm{~Hz}), 128.3,128.2(\mathrm{~d}, J=7.1 \mathrm{~Hz}), 127.9$, 122.0, 115.0, $88.9(\mathrm{~d}, J=4.0 \mathrm{~Hz}), 88.0,77.9,77.8,43.5(\mathrm{~d}, J=5.1 \mathrm{~Hz}), 30.0(\mathrm{~d}, J=2.0 \mathrm{~Hz}), 29.2$. ${ }^{31}$ P NMR (162 MHz, $\left.\mathrm{CDCl}_{3}\right) \delta 27.68$ (s). HRMS (ESI): ([M+H] $]^{+}$) Calcd for $\mathrm{C}_{26} \mathrm{H}_{26} \mathrm{O}_{2} \mathrm{P}: 401.1670$, Found: 401.1664. IR (film) v 2926, 2200, 1704, 1437, 1776, 1128, 954, 754, 726, $689 \mathrm{~cm}^{-1}$.

\section{4-(4-bromophenyl)-2-methylbut-3-yn-2-yl diphenylphosphinate (2ag)}

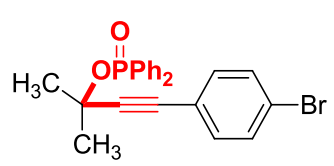

A white solid, m.p.: $185.3-186.1{ }^{\circ} \mathrm{C},(56.1 \mathrm{mg}, 64 \%$ yield $) .{ }^{1} \mathbf{H}$ NMR $\left(400 \mathrm{MHz}, \mathrm{CDCl}_{3}\right) \delta 7.90-$ 
$7.76(\mathrm{~m}, 4 \mathrm{H}), 7.49-7.34(\mathrm{~m}, 6 \mathrm{H}), 7.25-7.16(\mathrm{~m}, 2 \mathrm{H}), 7.03(\mathrm{~d}, J=4.0 \mathrm{~Hz}, 2 \mathrm{H}), 1.90(\mathrm{~s}, 6 \mathrm{H}) .{ }^{13} \mathrm{C}$ NMR $\left(101 \mathrm{MHz}, \mathrm{CDCl}_{3}\right) \delta 134.5,133.2,131.6,131.6,131.6,131.5,131.5,128.4,128.3,128.2$, 127.9, 122.1, 90.2, 86.5, $75.1(\mathrm{~d}, J=8.1 \mathrm{~Hz}), 32.1(\mathrm{~d}, J=4.0 \mathrm{~Hz}) .{ }^{31} \mathbf{P}$ NMR $\left(162 \mathrm{MHz}, \mathrm{CDCl}_{3}\right) \delta$ 27.81 (s). HRMS (ESI): $\left([\mathrm{M}+\mathrm{Na}]^{+}\right)$Calcd for $\mathrm{C}_{23} \mathrm{H}_{20} \mathrm{BrNaO}_{2} \mathrm{P}: 461.0282$, Found: 461.0276. IR (film) v 2919, 2849, 1645, 1437, 1177, 1128, 956, 726, $690 \mathrm{~cm}^{-1}$.

\section{4-(4-fluorophenyl)-2-methylbut-3-yn-2-yl diphenylphosphinate (2ah)}

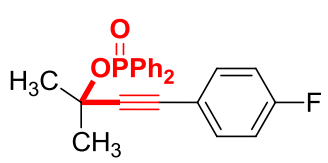

A white solid, m.p.: $189.5-190.7^{\circ} \mathrm{C},(36.3 \mathrm{mg}, 48 \%$ yield $) .{ }^{1} \mathbf{H}$ NMR $\left(400 \mathrm{MHz}, \mathrm{CDCl}_{3}\right) \delta 7.88-$ $7.76(\mathrm{~m}, 4 \mathrm{H}), 7.47-7.35(\mathrm{~m}, 6 \mathrm{H}), 7.01-6.94(\mathrm{~m}, 2 \mathrm{H}), 6.92-6.83(\mathrm{~m}, 2 \mathrm{H}), 1.89(\mathrm{~s}, 6 \mathrm{H}) .{ }^{13} \mathbf{C}$ NMR $\left(101 \mathrm{MHz}, \mathrm{CDCl}_{3}\right) \delta 162.6(\mathrm{~d}, J=250.5 \mathrm{~Hz}), 133.8(\mathrm{~d}, J=139.4 \mathrm{~Hz}), 133.6(\mathrm{~d}, J=8.1 \mathrm{~Hz}), 133.6$, 131.5, $128.3(\mathrm{~d}, J=13.1 \mathrm{~Hz}), 118.1,115.2(\mathrm{~d}, J=22.2 \mathrm{~Hz}), 89.9,85.7,74.9(\mathrm{~d}, J=8.1 \mathrm{~Hz}), 32.1(\mathrm{~d}$, $J=4.1 \mathrm{~Hz}) \cdot{ }^{31} \mathrm{P}$ NMR $\left(162 \mathrm{MHz}, \mathrm{CDCl}_{3}\right) \delta 27.90$ (s). HRMS (ESI): $\left([\mathrm{M}+\mathrm{Na}]^{+}\right)$Calcd for $\mathrm{C}_{23} \mathrm{H}_{20} \mathrm{FNaO}_{2} \mathrm{P}: 401.1082$, Found: 401.1077. IR (film) v 2920, 2157, 2015, 1588, 1437, 1177, 975, $931,726,690 \mathrm{~cm}^{-1}$.

\section{2-methyl-4-(p-tolyl)but-3-yn-2-yl diphenylphosphinate (2ai)}

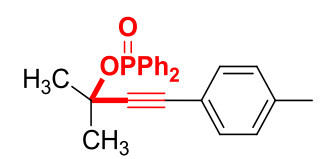

A white solid, m.p.: $90.1-90.9{ }^{\circ} \mathrm{C},(53.9 \mathrm{mg}, 72 \%$ yield $) .{ }^{1} \mathbf{H}$ NMR $\left(400 \mathrm{MHz}, \mathrm{CDCl}_{3}\right) \delta 7.87-$ 7.77 (m, 4H), $7.46-7.34$ (m, 6H), 7.00 (d, $J=7.8 \mathrm{~Hz}, 2 \mathrm{H}), 6.91$ (d, $J=7.8 \mathrm{~Hz}, 2 \mathrm{H}), 2.30(\mathrm{~s}, 3 \mathrm{H})$, $1.89(\mathrm{~s}, 6 \mathrm{H}) .{ }^{13} \mathbf{C}$ NMR $\left(101 \mathrm{MHz}, \mathrm{CDCl}_{3}\right) \delta 138.5,134.6,133.2,131.6,131.5(\mathrm{~d}, J=1.0 \mathrm{~Hz}), 128.6$, $128.3(\mathrm{~d}, J=13.1 \mathrm{~Hz}), 119.0,89.5(\mathrm{~d}, J=4.0 \mathrm{~Hz}), 86.7,75.2(\mathrm{~d}, J=8.1 \mathrm{~Hz}), 32.2(\mathrm{~d}, J=4.0 \mathrm{~Hz})$, 21.5. ${ }^{31}$ P NMR $\left(162 \mathrm{MHz}, \mathrm{CDCl}_{3}\right) \delta 27.69$ (s). HRMS (ESI): $\left([\mathrm{M}+\mathrm{Na}]^{+}\right)$Calcd for $\mathrm{C}_{24} \mathrm{H}_{23} \mathrm{NaO}_{2} \mathrm{P}$ : 397.1333, Found: 397.1327. IR (film) v 2919, 2847, 2158, 1976, 1623, 1594, 1436, 1289, 1139, $1114,1082,746,693 \mathrm{~cm}^{-1}$.

\section{2-ethoxy-4-iodo-5,5-dimethyl-3-phenyl-5H-1,2-oxaphosphole 2-oxide (2")}




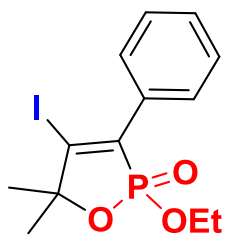

Pale yellow oil, $73 \%$ yield. ${ }^{1} \mathbf{H}$ NMR $\left(400 \mathrm{MHz} \mathrm{CDCl}_{3}\right) \delta 7.58(\mathrm{~d}, J=7.1 \mathrm{~Hz}, 2 \mathrm{H}), 7.52-7.35(\mathrm{~m}$, 3H), $4.24-3.94(\mathrm{~m}, 2 \mathrm{H}), 1.68(\mathrm{~s}, 3 \mathrm{H}), 1.63(\mathrm{~s}, 3 \mathrm{H}), 1.19(\mathrm{t}, J=7.1 \mathrm{~Hz}, 3 \mathrm{H}) .{ }^{13} \mathbf{C}$ NMR $(101 \mathrm{MHz}$, $\left.\mathrm{CDCl}_{3}\right) \delta 135.6(\mathrm{~d}, J=149.4 \mathrm{~Hz}), 132.3(\mathrm{~d}, J=10.8 \mathrm{~Hz}), 129.1,128.7,128.1(\mathrm{~d}, J=5.5 \mathrm{~Hz}), 124.2$ $(\mathrm{d}, J=43.4 \mathrm{~Hz}), 87.7(\mathrm{~d}, J=5.8 \mathrm{~Hz}), 63.8(\mathrm{~d}, J=6.8 \mathrm{~Hz}), 28.3(\mathrm{~d}, J=2.6 \mathrm{~Hz}), 28.3(\mathrm{~d}, J=1.4 \mathrm{~Hz})$, $16.5(\mathrm{~d}, J=5.5 \mathrm{~Hz}) .{ }^{31} \mathbf{P}$ NMR $\left(162 \mathrm{MHz}, \mathrm{CDCl}_{3}\right) \delta 28.81$ (s). HRMS (ESI): $\left([\mathrm{M}+\mathrm{H}]^{+}\right)$Calcd for $\mathrm{C}_{13} \mathrm{H}_{17} \mathrm{IO}_{3} \mathrm{P}: 378.9960$, Found: 378.9948 .

\section{3. ${ }^{1} \mathrm{H} /{ }^{13} \mathrm{C}-\mathrm{NMR} /{ }^{31} \mathrm{P}-\mathrm{NMR}$ and HRMS Spectra of Substrate and Products}

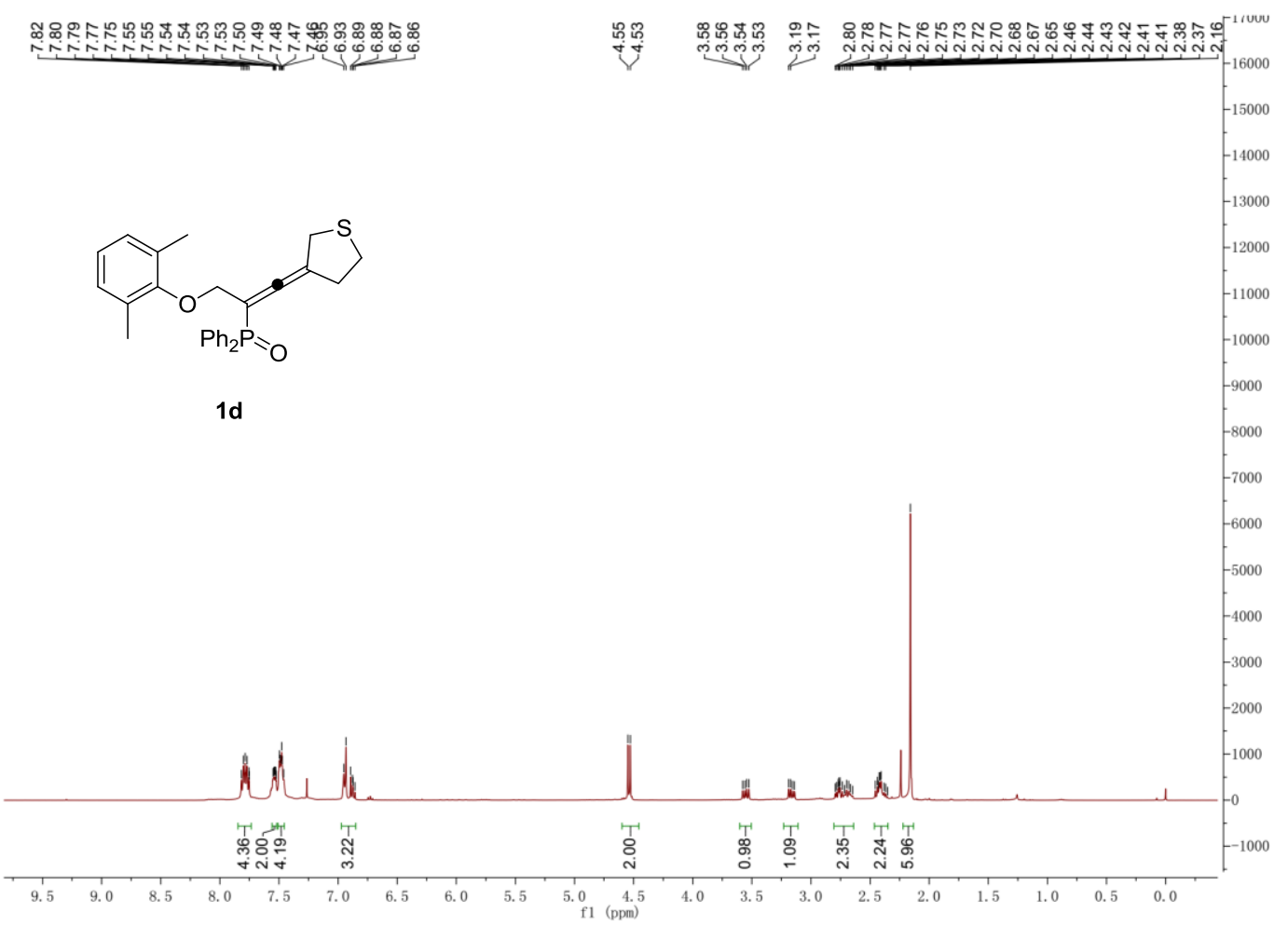




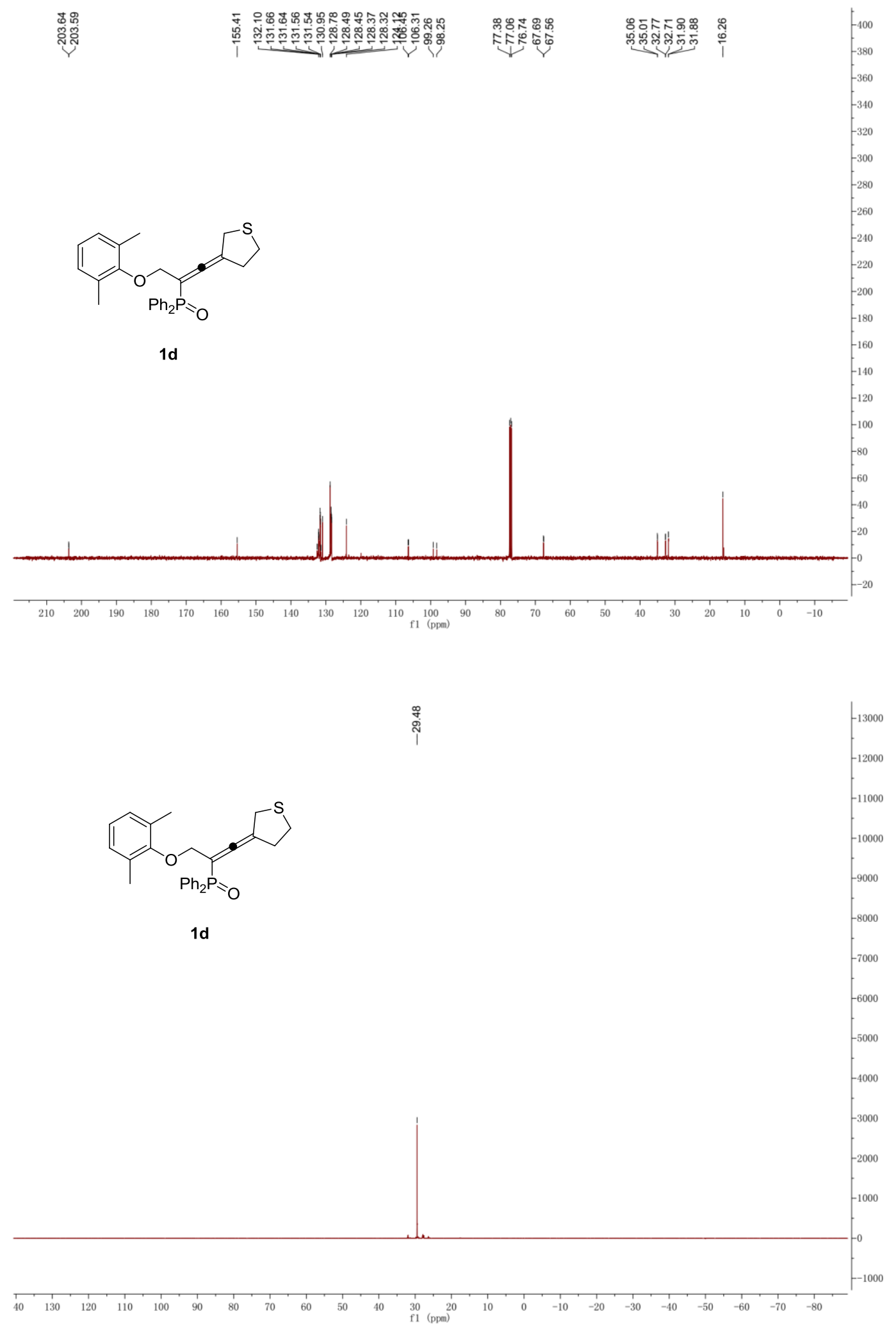


20160913-22 \#51-52 RT: 0.52-0.53 AV: 2 NL: $2.73 E 5$

T: FTMS $\{1,1\}+p$ ESI Full ms [100.00-1000.00]

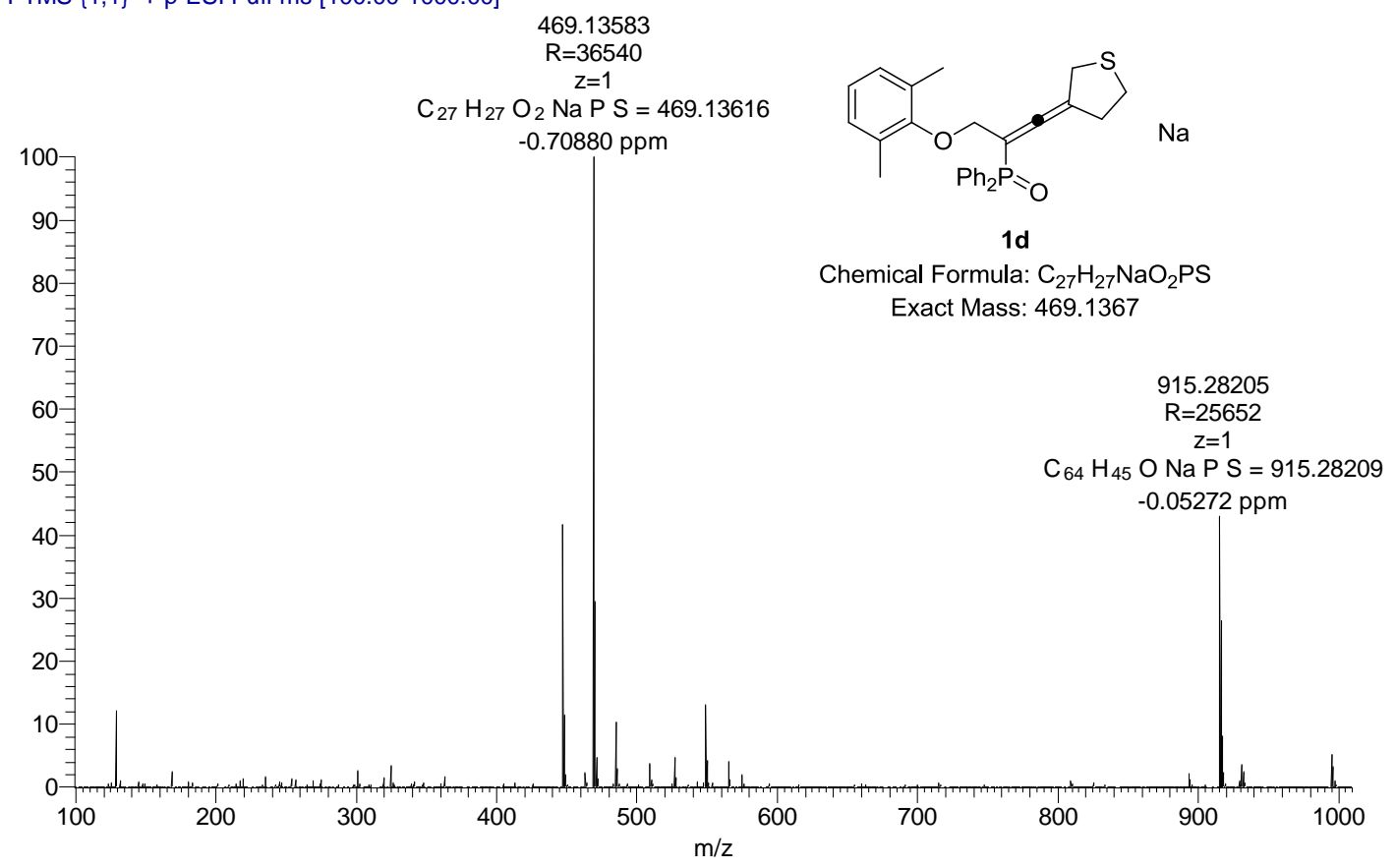

\section{兽}

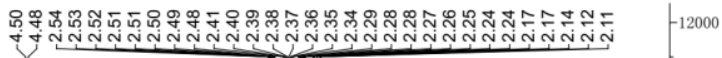

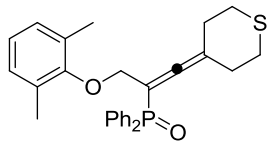

1e

(in 


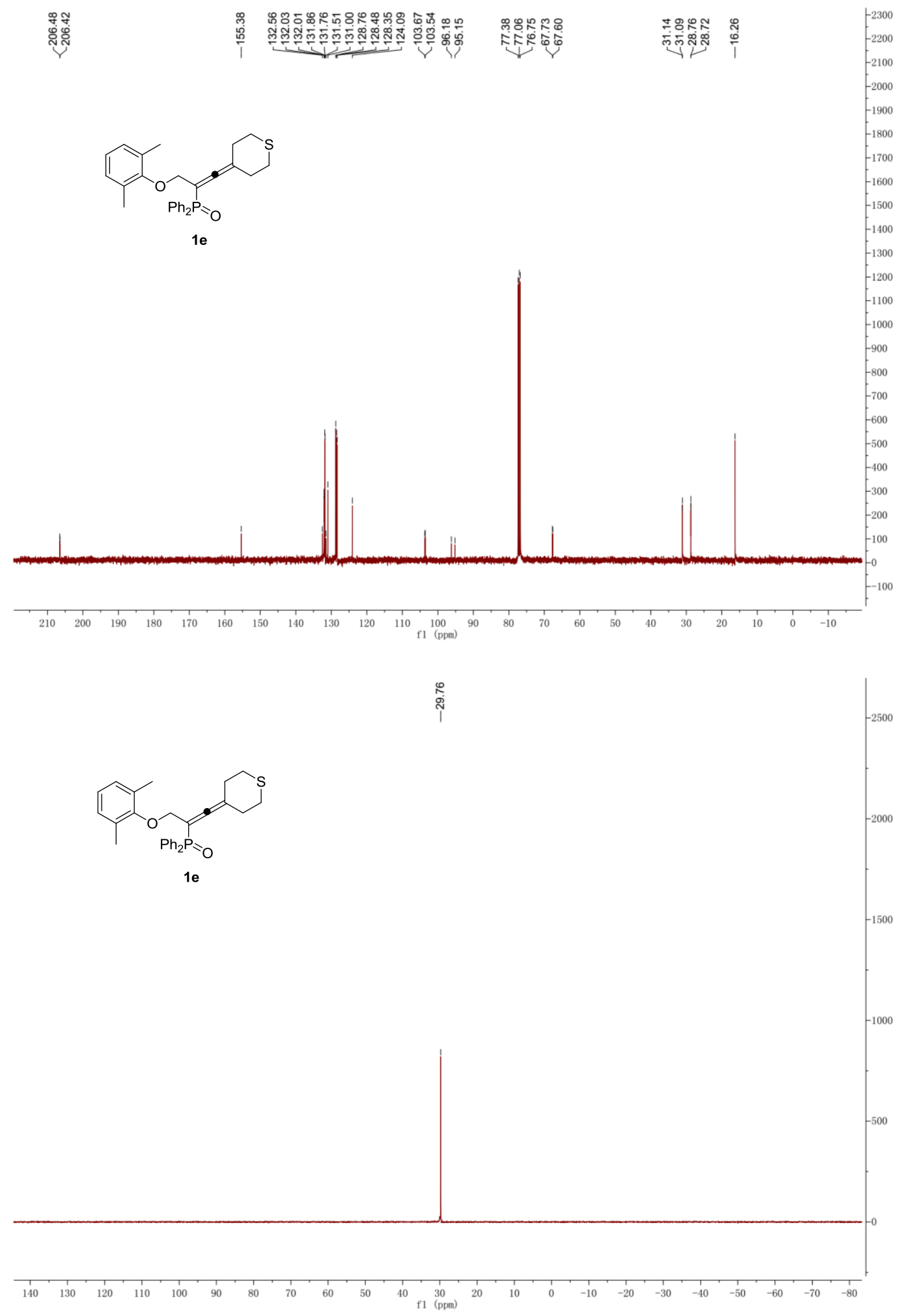


1 \#47-48 RT: 0.46-0.47 AV: 2 NL: 1.29E6

T: FTMS $\{1,1\}+p$ ESI Full ms [100.00-1000.00]

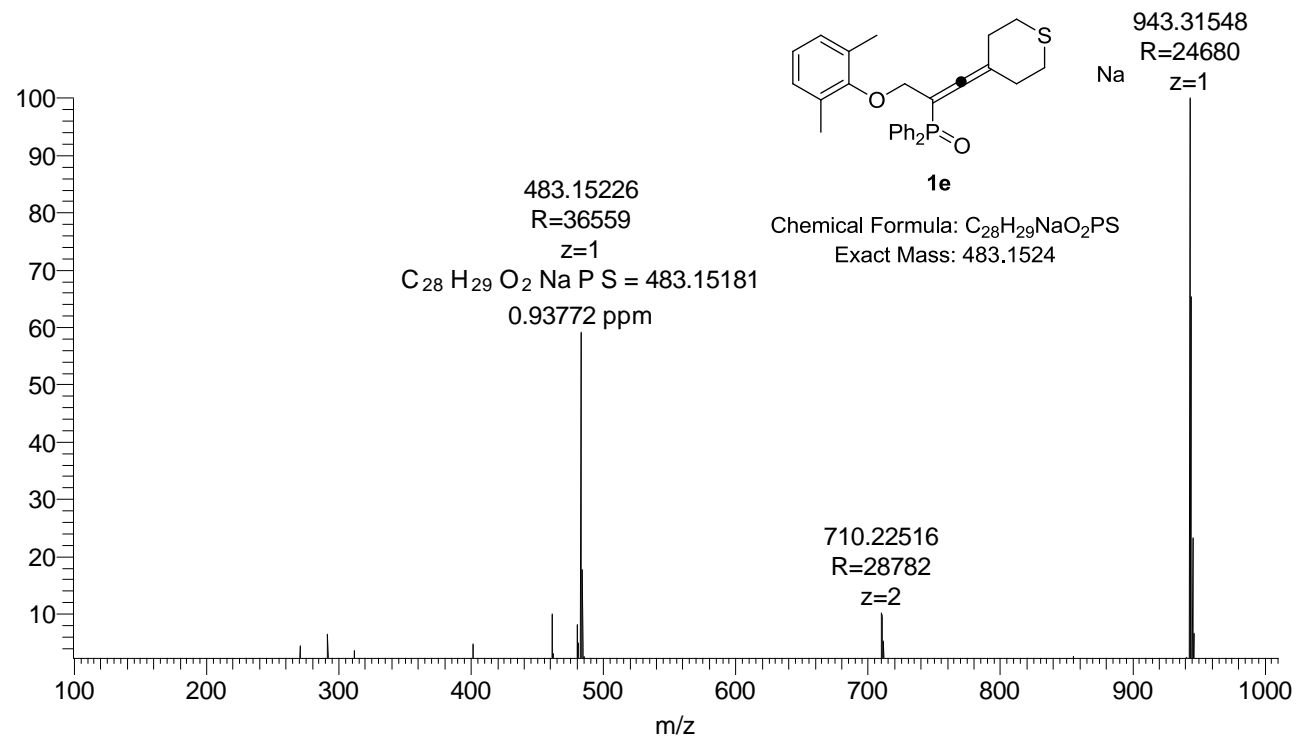

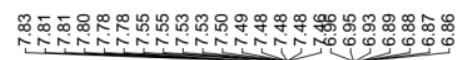

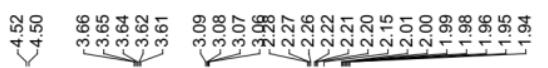
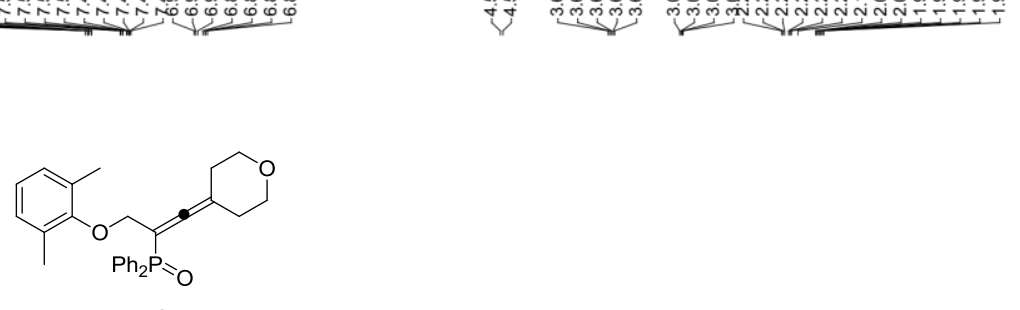

$-38000$

$1 f$

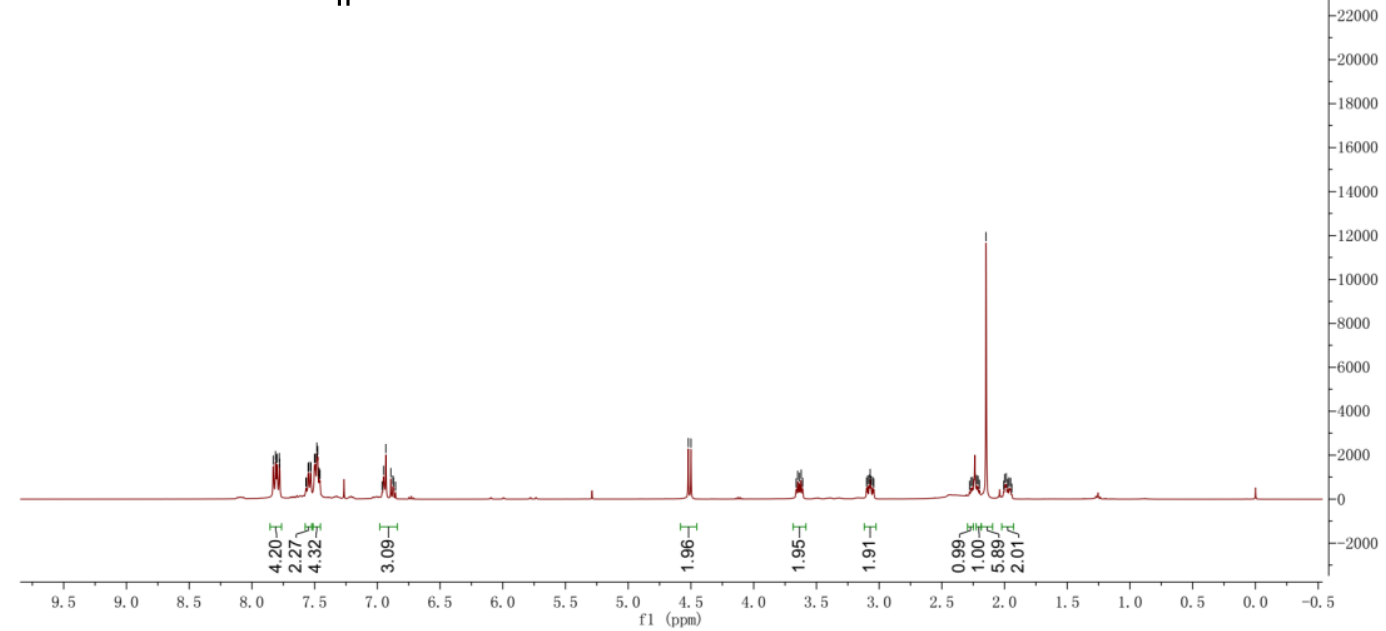




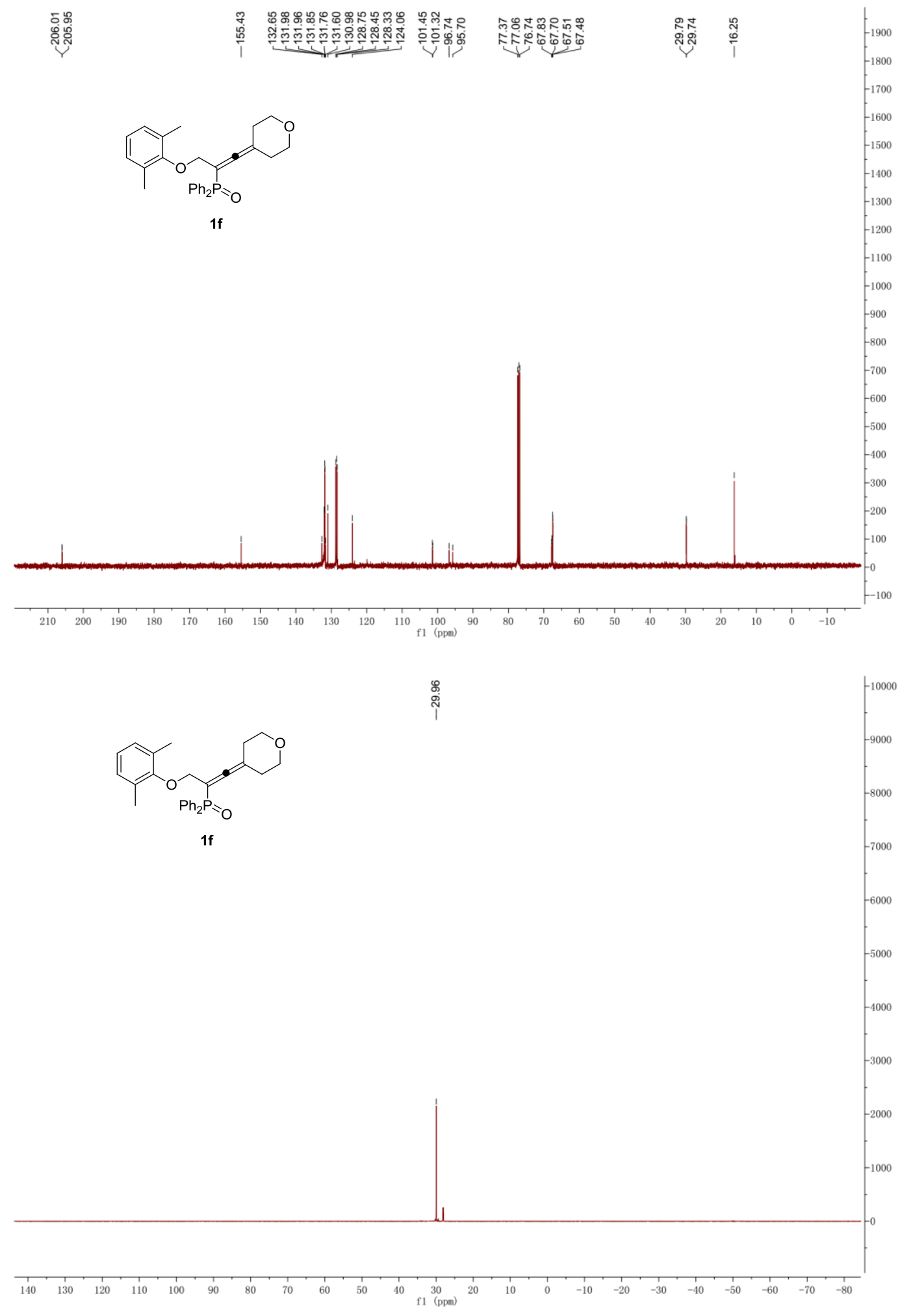


20160913-21 \#53 RT: 0.52 AV: 1 NL: 1.74E5

T: FTMS $\{1,1\}+p$ ESI Full ms [100.00-1000.00]

467.17450

$$
\mathrm{R}=38000
$$

$\mathrm{C}_{28} \mathrm{H}_{29} \mathrm{O}_{3} \mathrm{NaP}=467.17465$

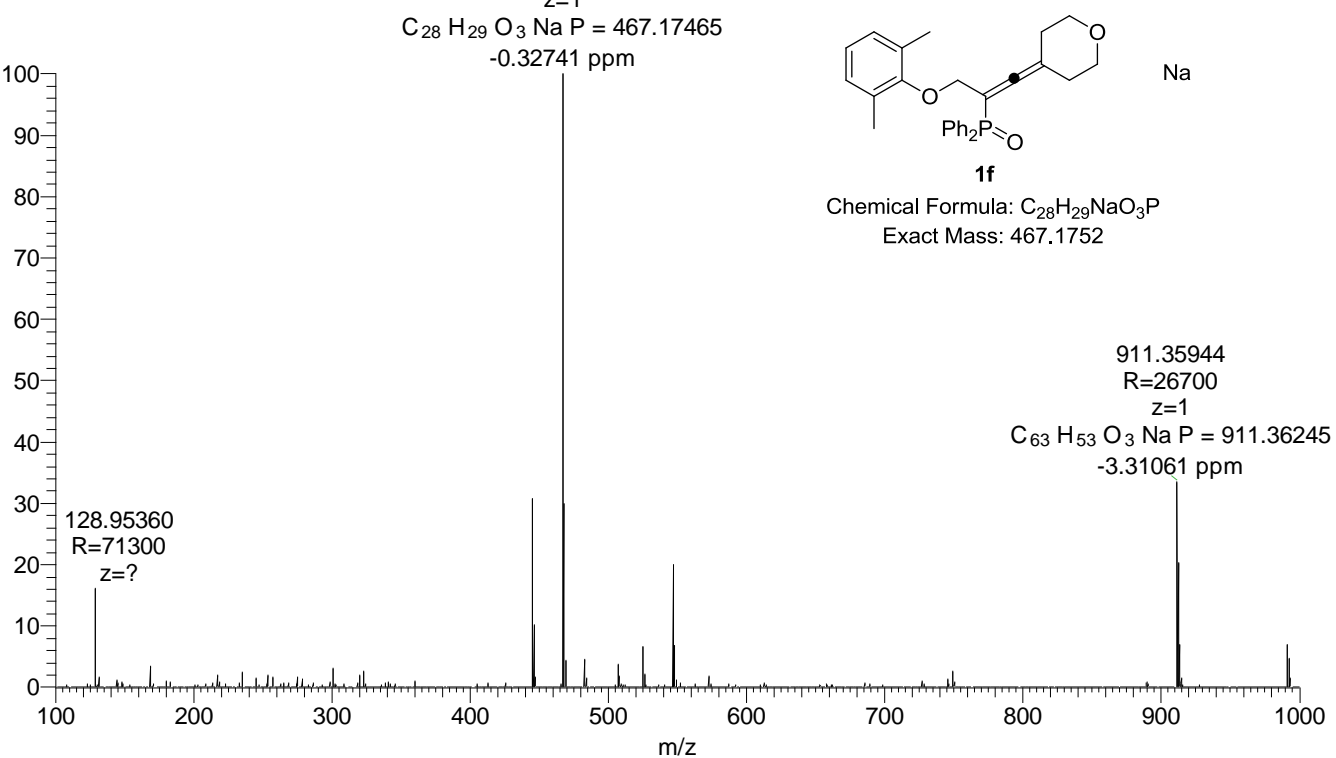

emical Formula: $\mathrm{C}_{28} \mathrm{H}_{29} \mathrm{NaO}_{3} \mathrm{P}$ Exact Mass: 467.1752

$$
\mathrm{R}=26700
$$$$
3 \mathrm{NaP}=911.36245
$$

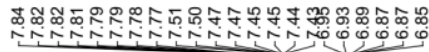<smiles>CCCCCC/C(C)=C/C(=P)P/C(=C/P)COc1c(C)cccc1C</smiles>

$1 \mathrm{i}$

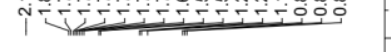

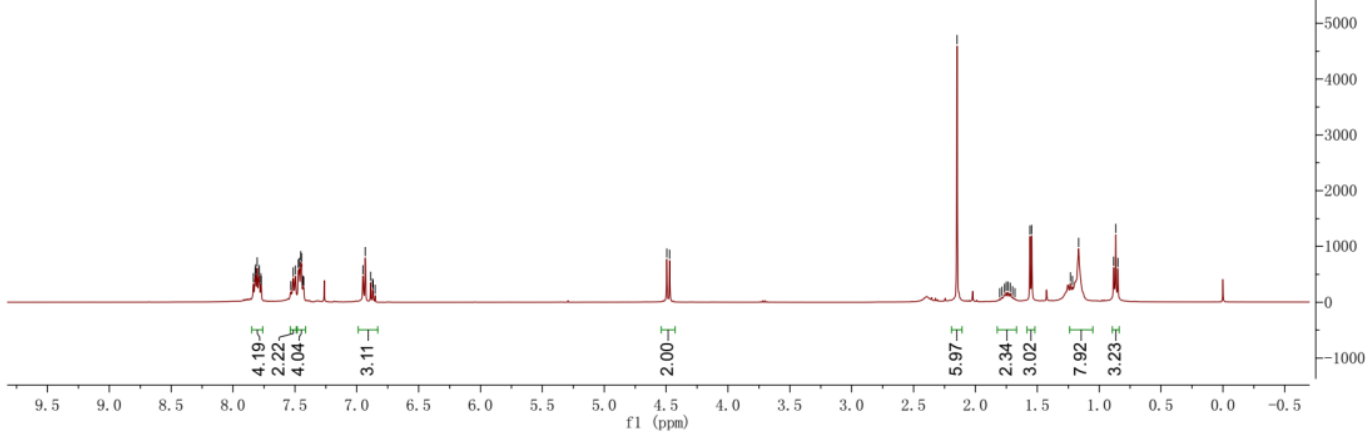




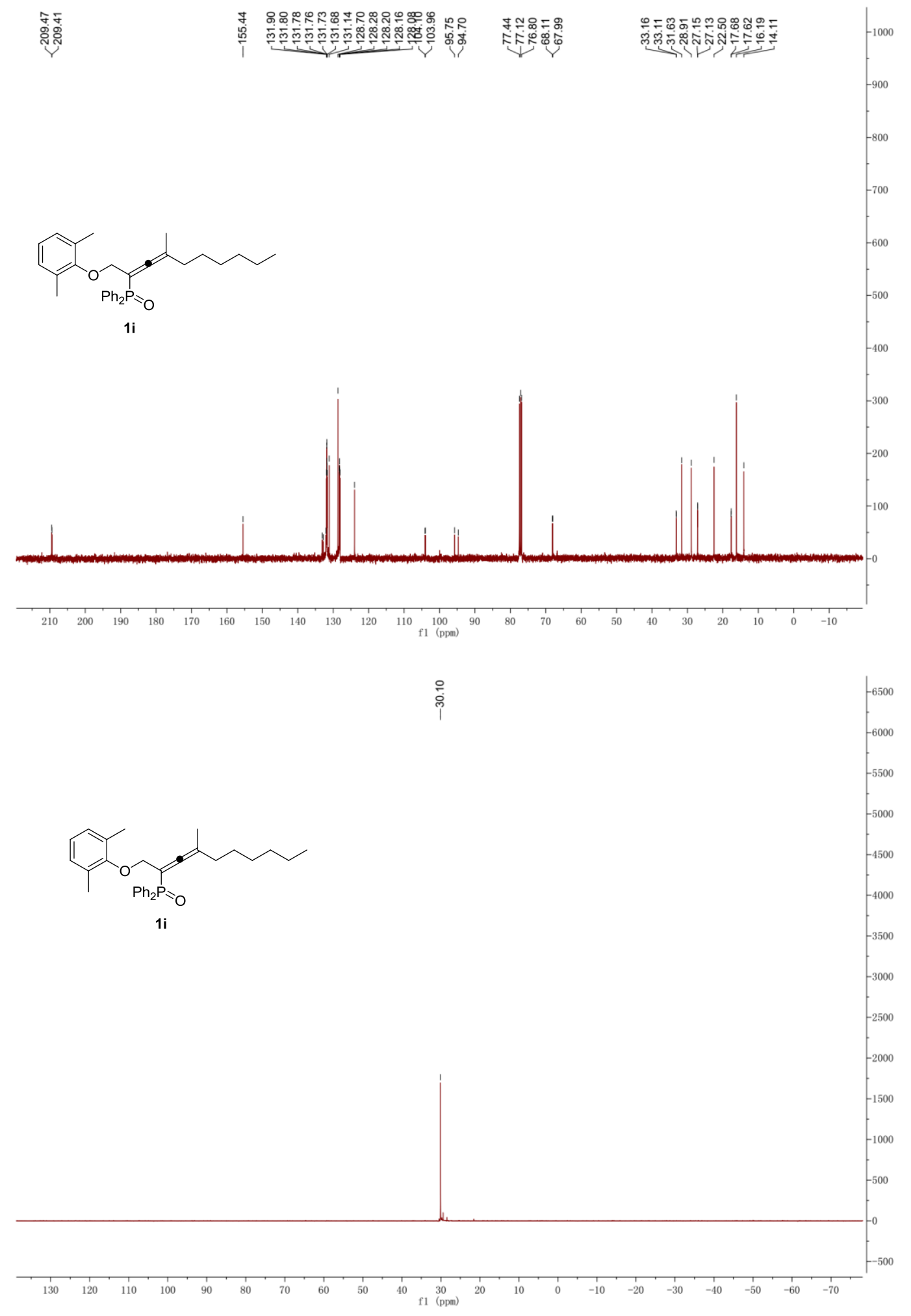



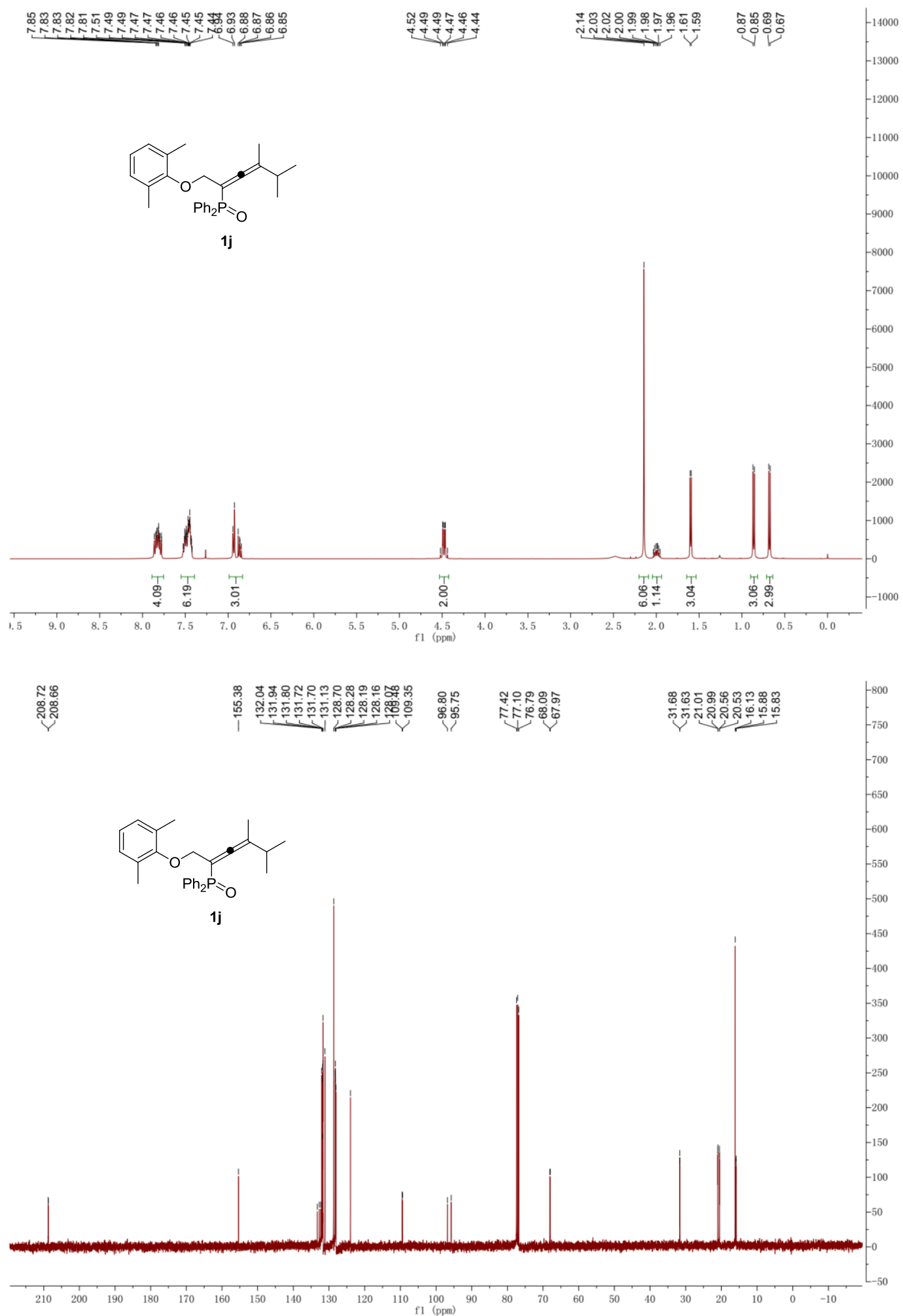


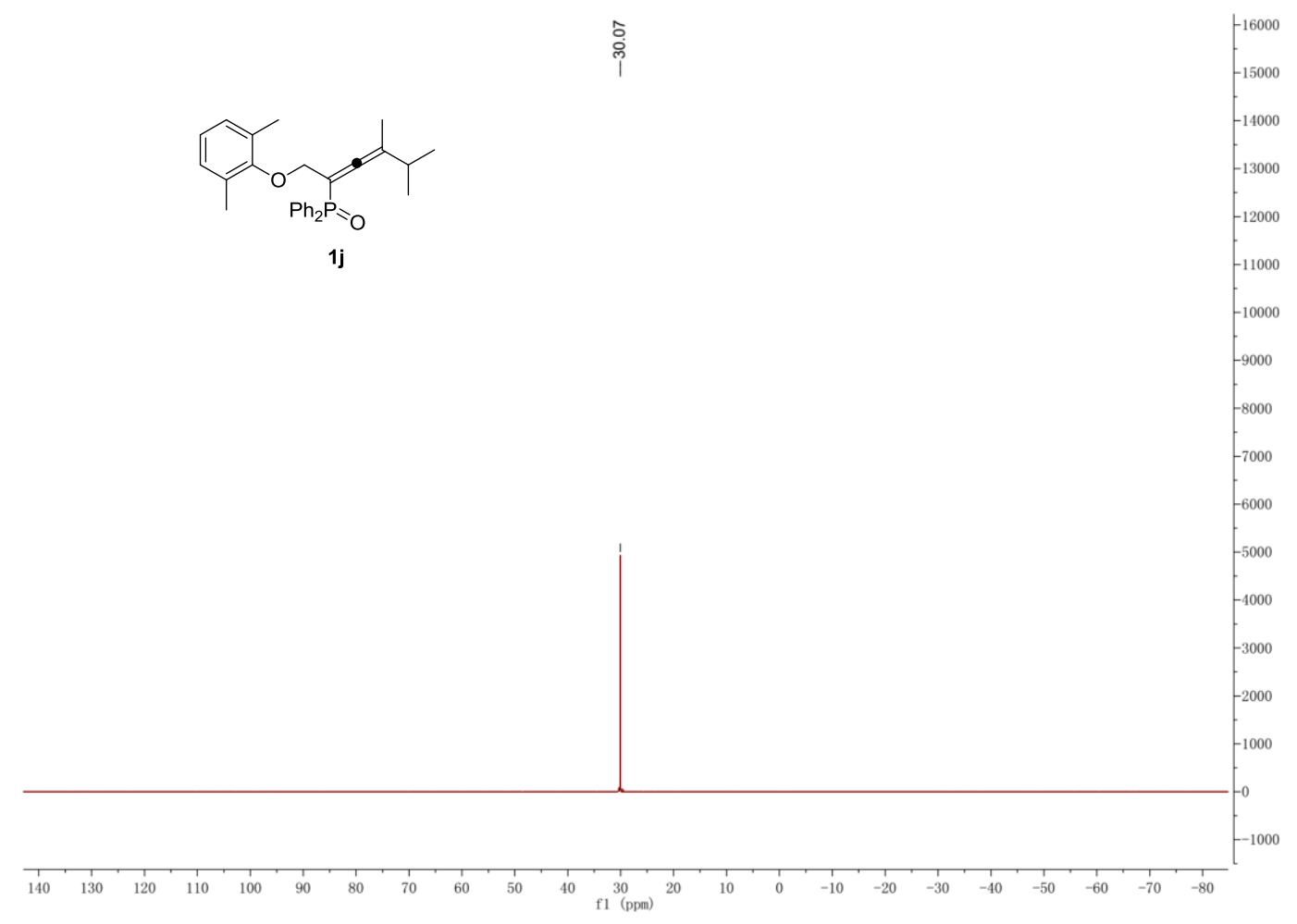

20160826-9 \#92-94 RT: 0.96-0.99 AV: 3 NL: 1.15E5 T: FTMS $\{1,1\}+p$ ESI Full ms [100.00-1000.00]

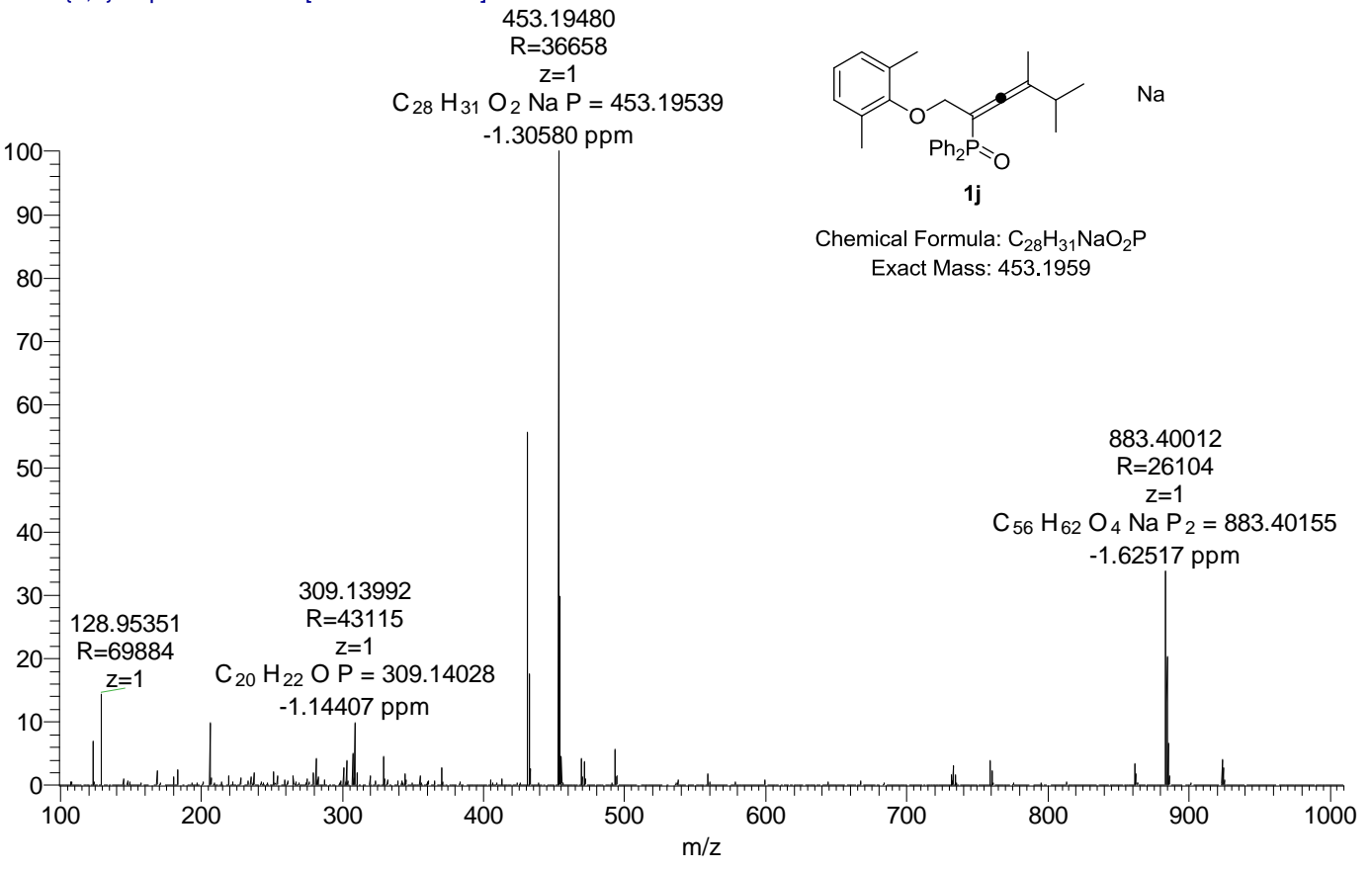




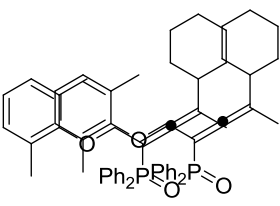

$1 \mathrm{k} \quad 1 \mathrm{k}$

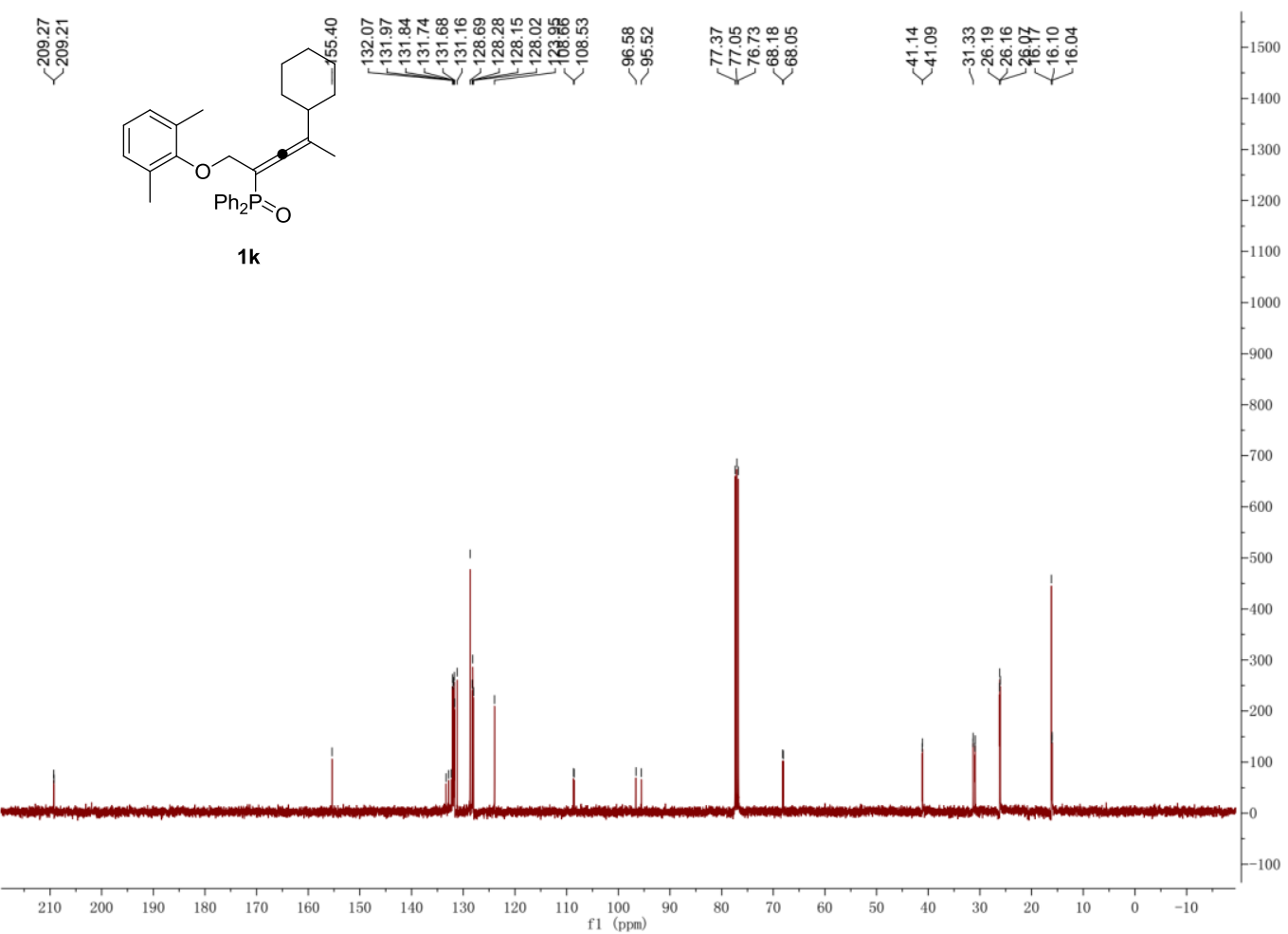




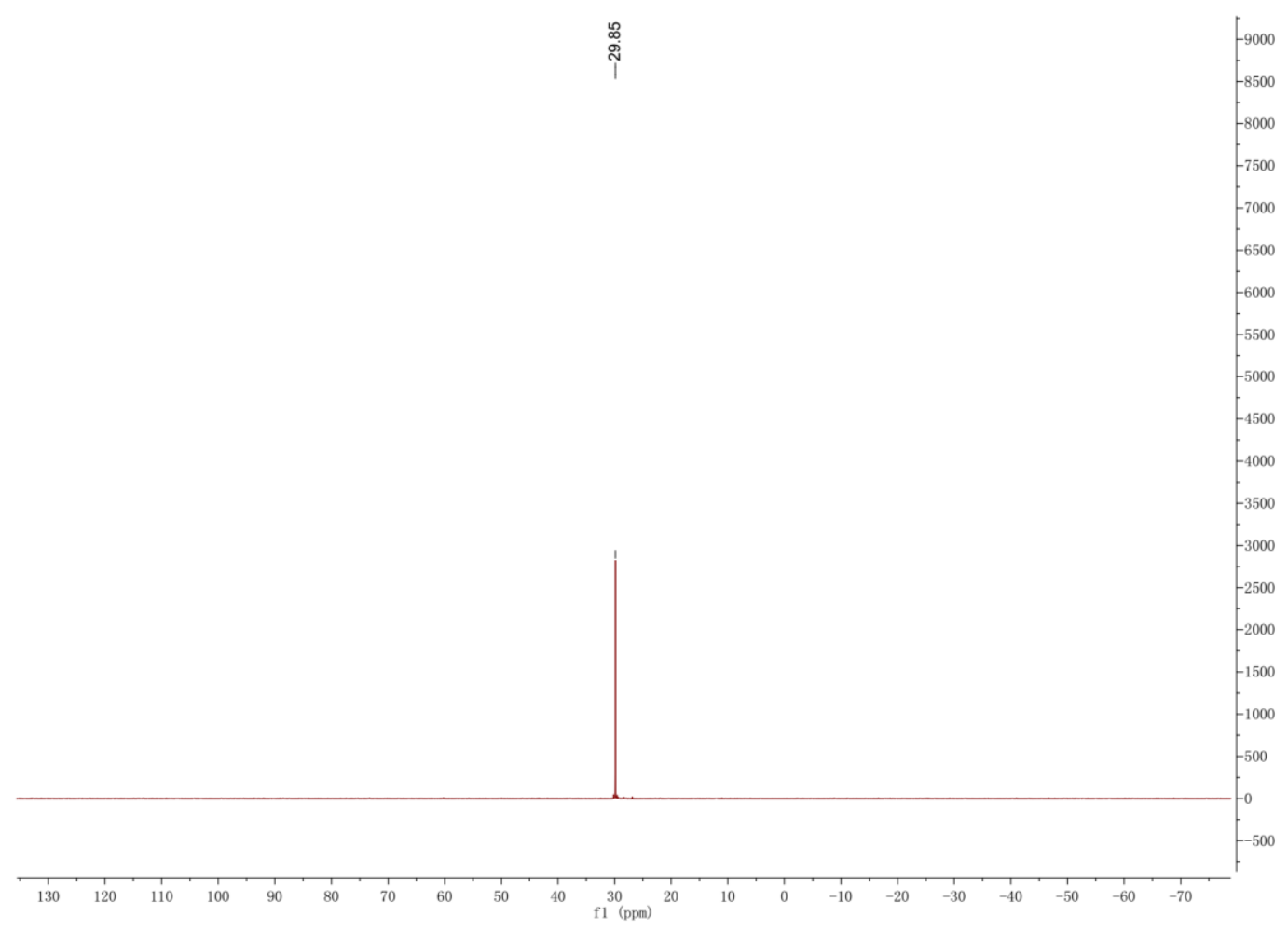

20160826-8 \#95 RT: 0.91 AV: 1 NL: 2.04E5 T: FTMS $\{1,1\}+p$ ESI Full ms [100.00-1000.00]

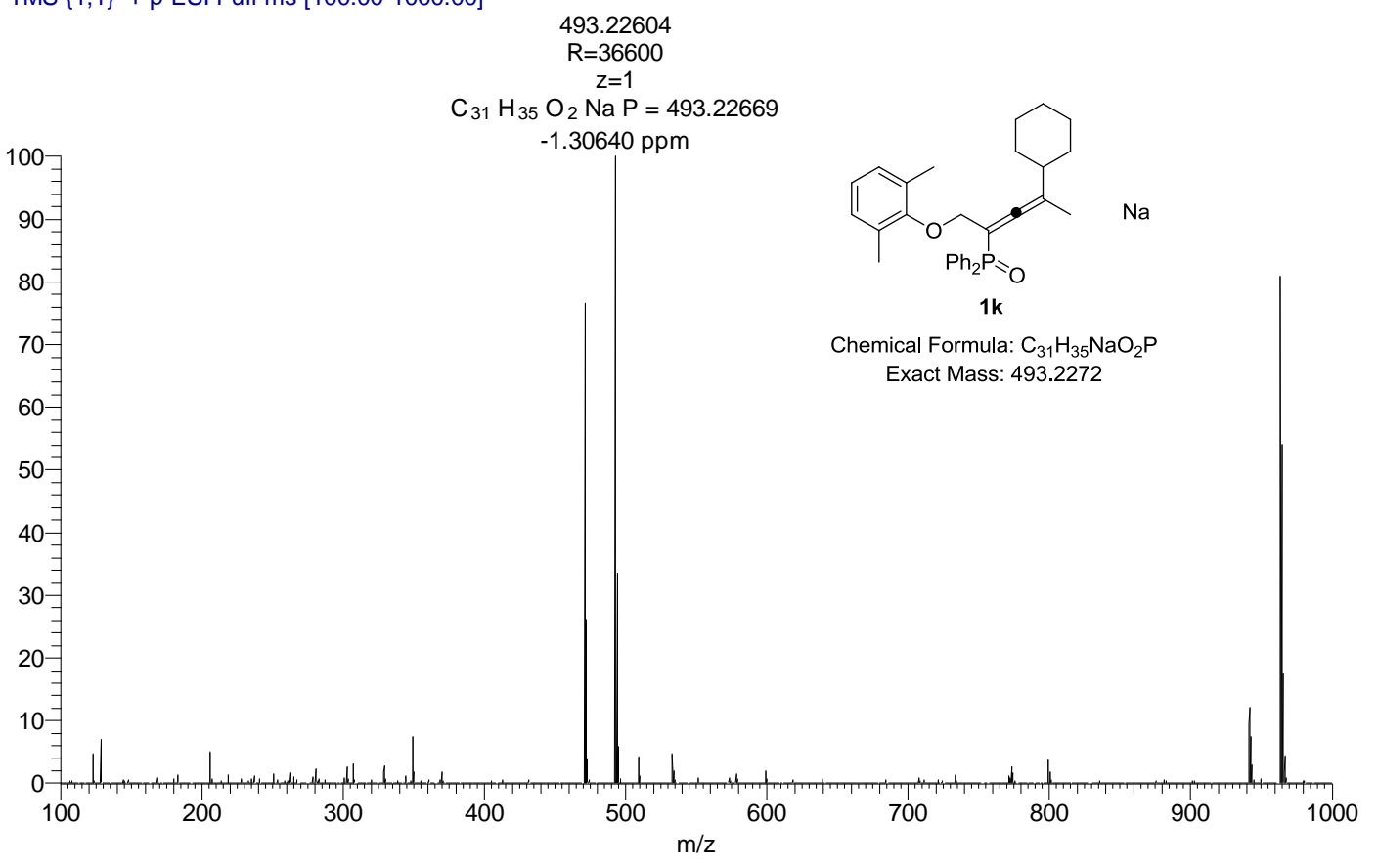




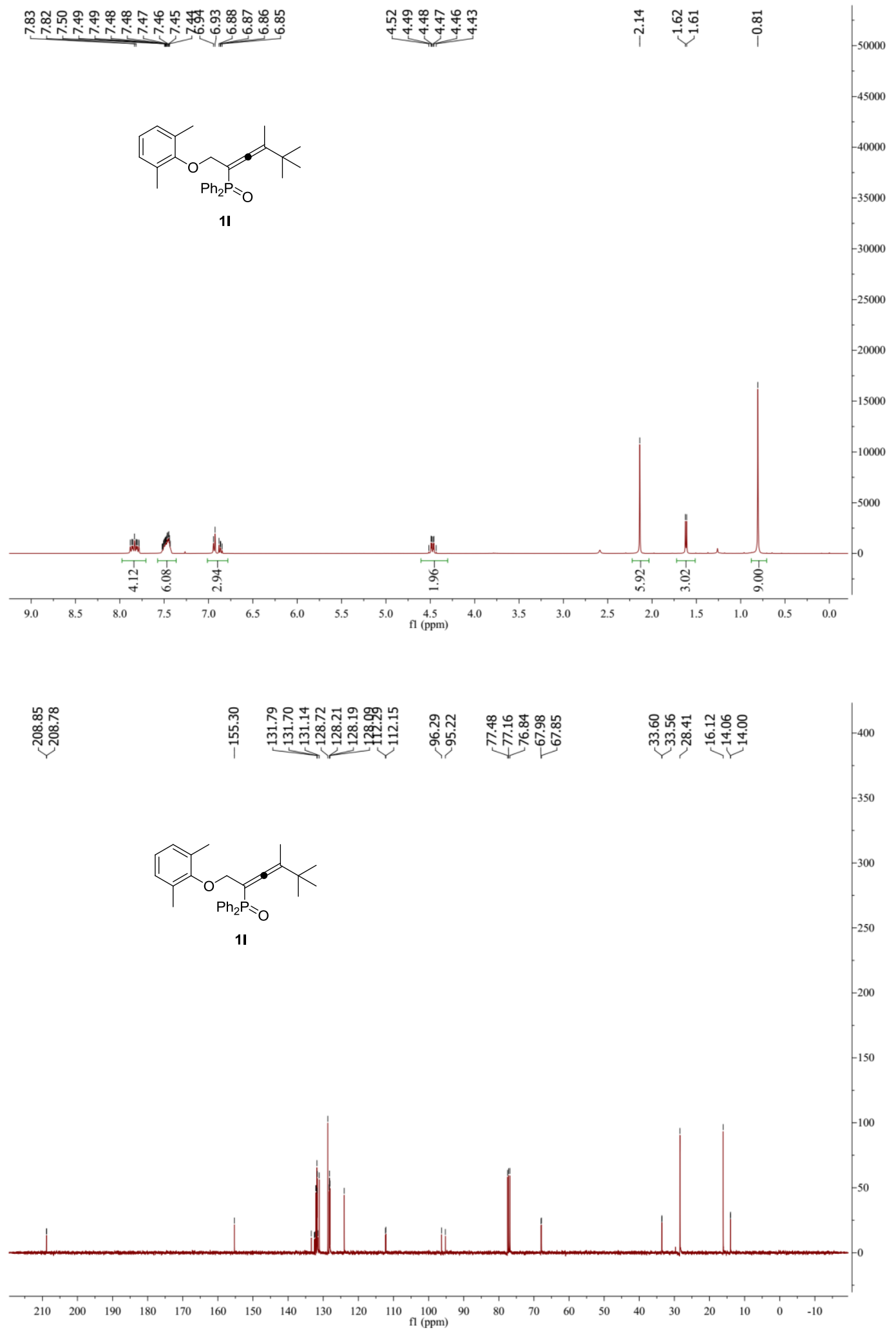




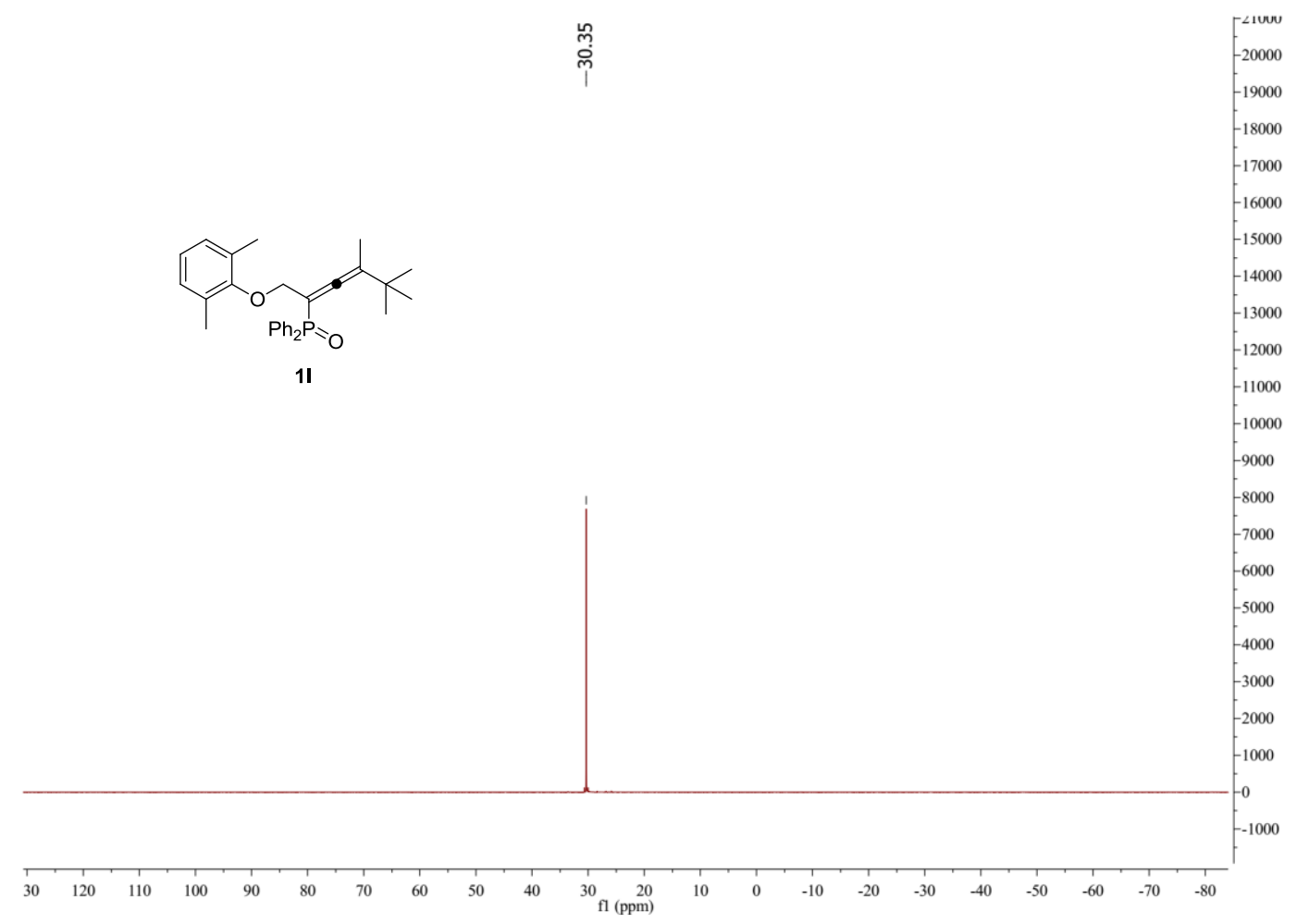

20160913-23 \#62 RT: 0.53 AV: 1 NL: 6.14E5 T: FTMS $\{1,1\}+p$ ESI Full ms $[100.00-1000.00]$

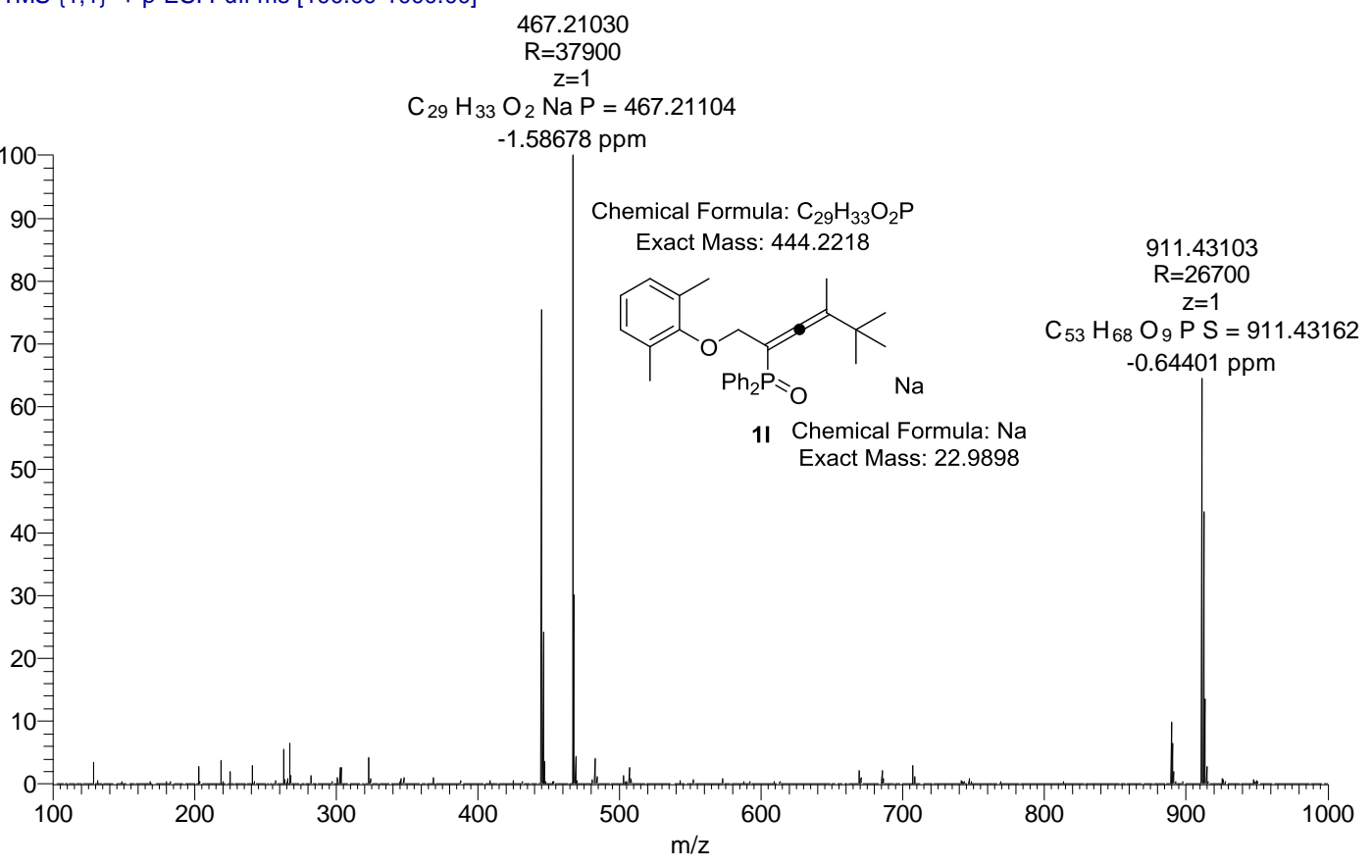




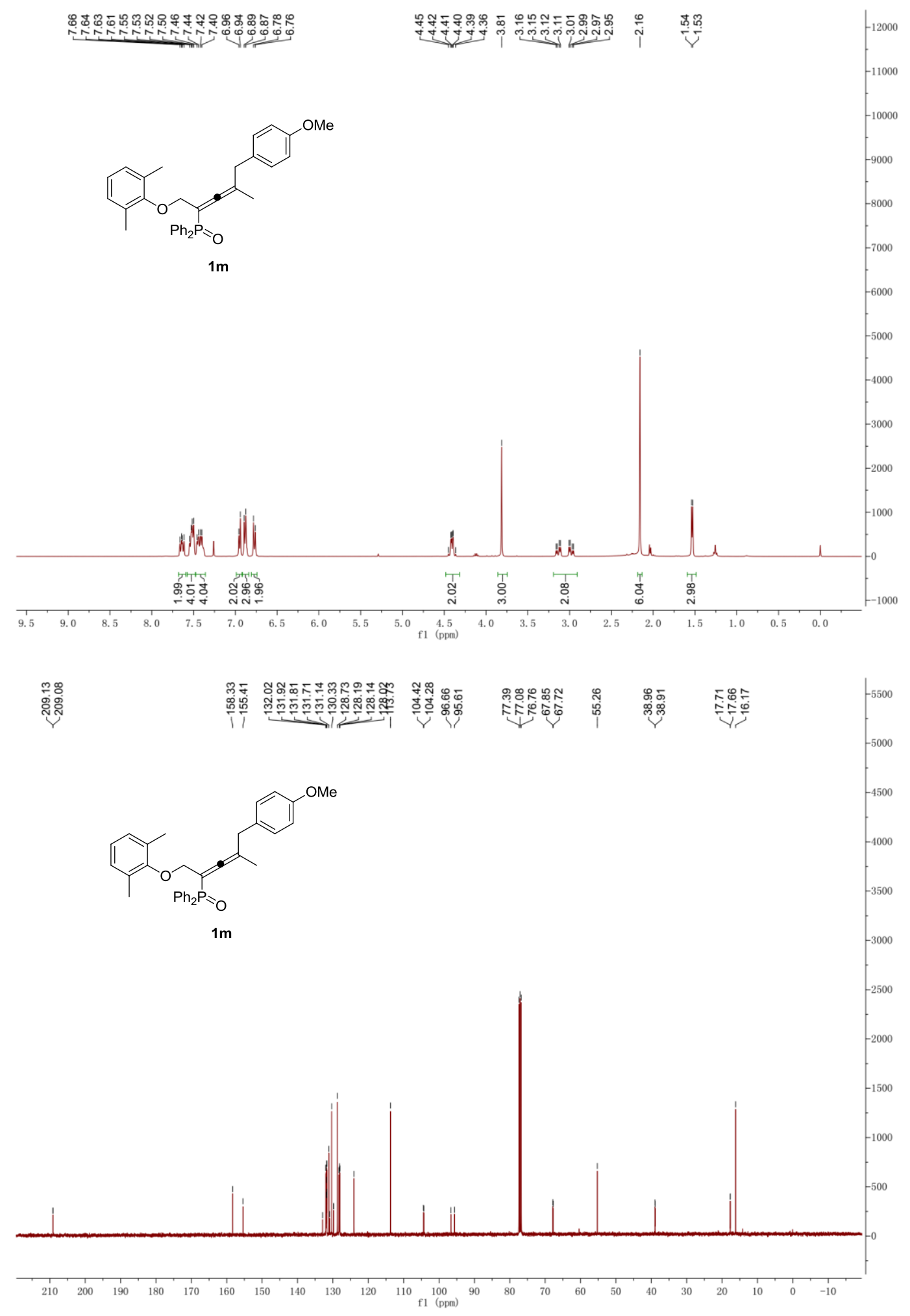




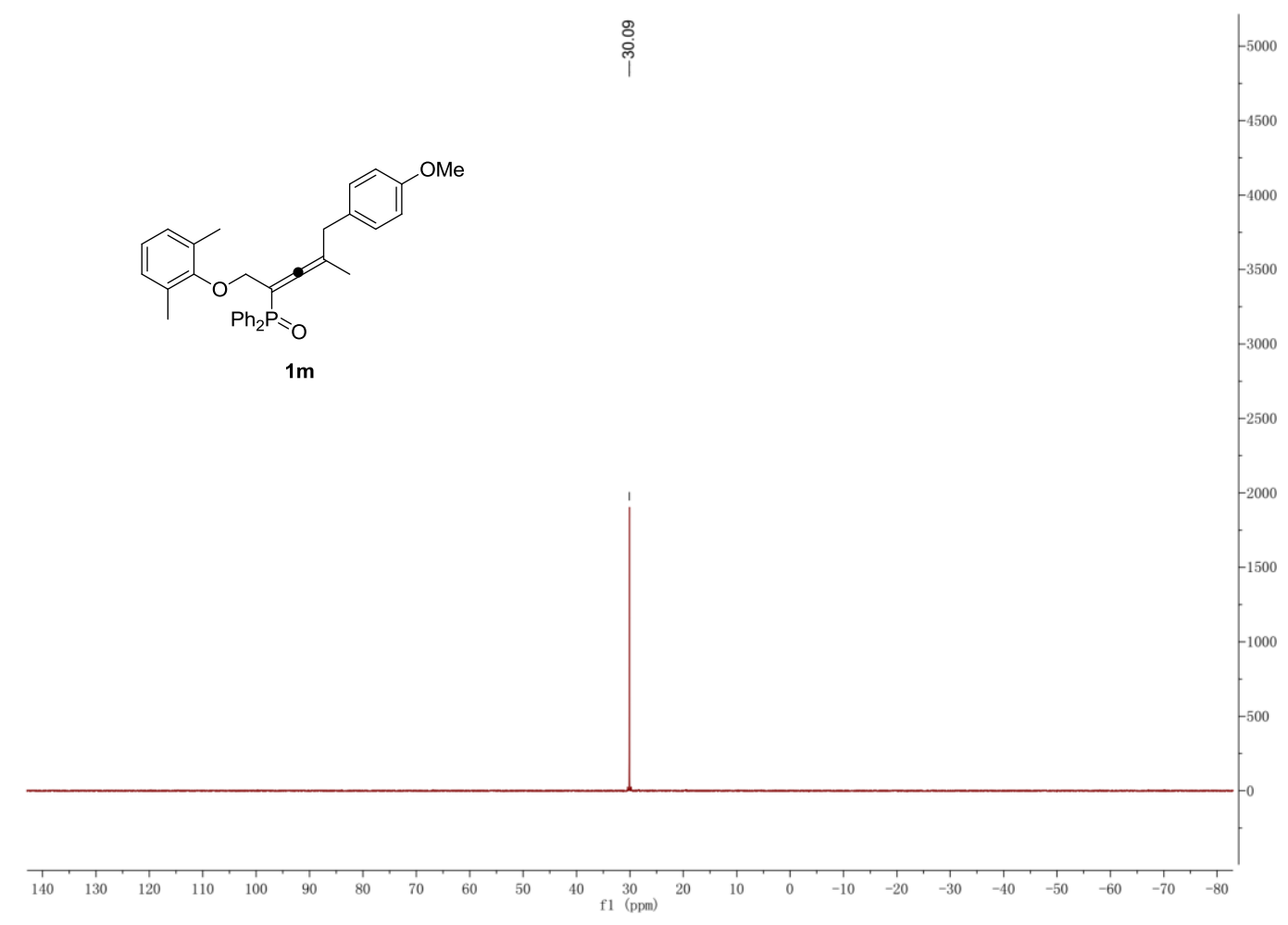

20160913-24 \#95-97 RT: 0.91-0.94 AV: 3 NL: 5.30E5 T: FTMS $\{1,1\}+p$ ESI Full ms [100.00-1000.00]

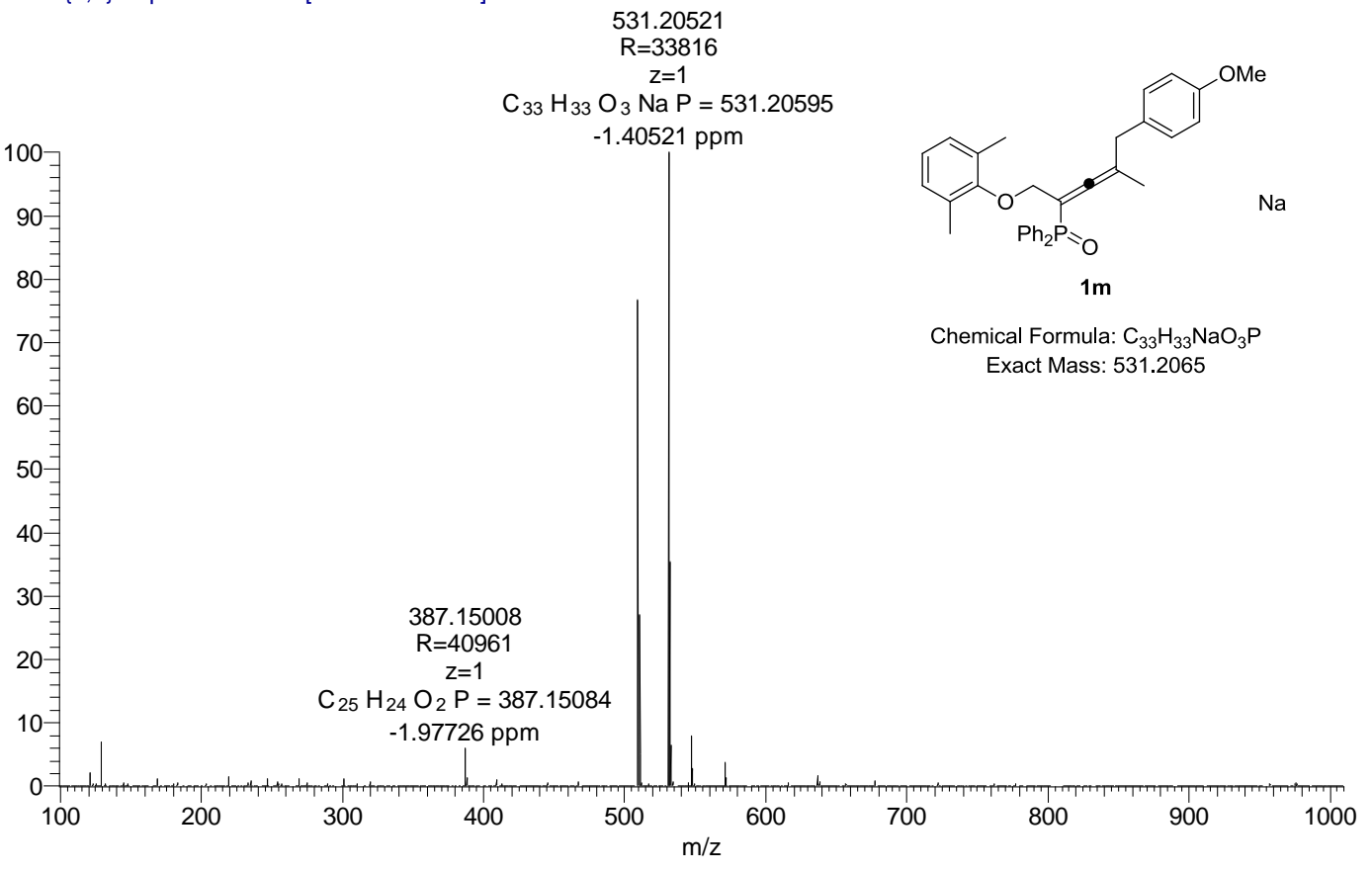



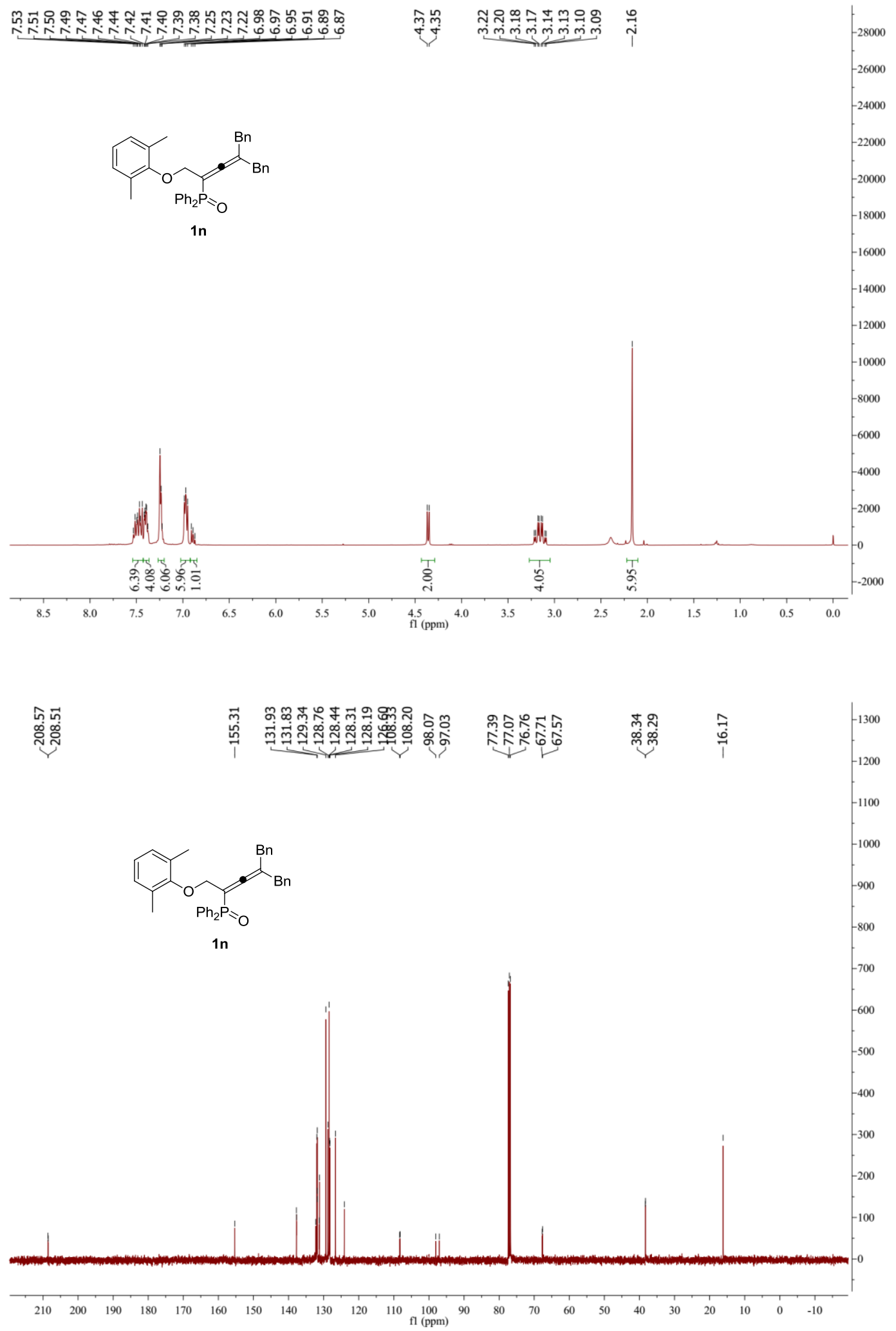


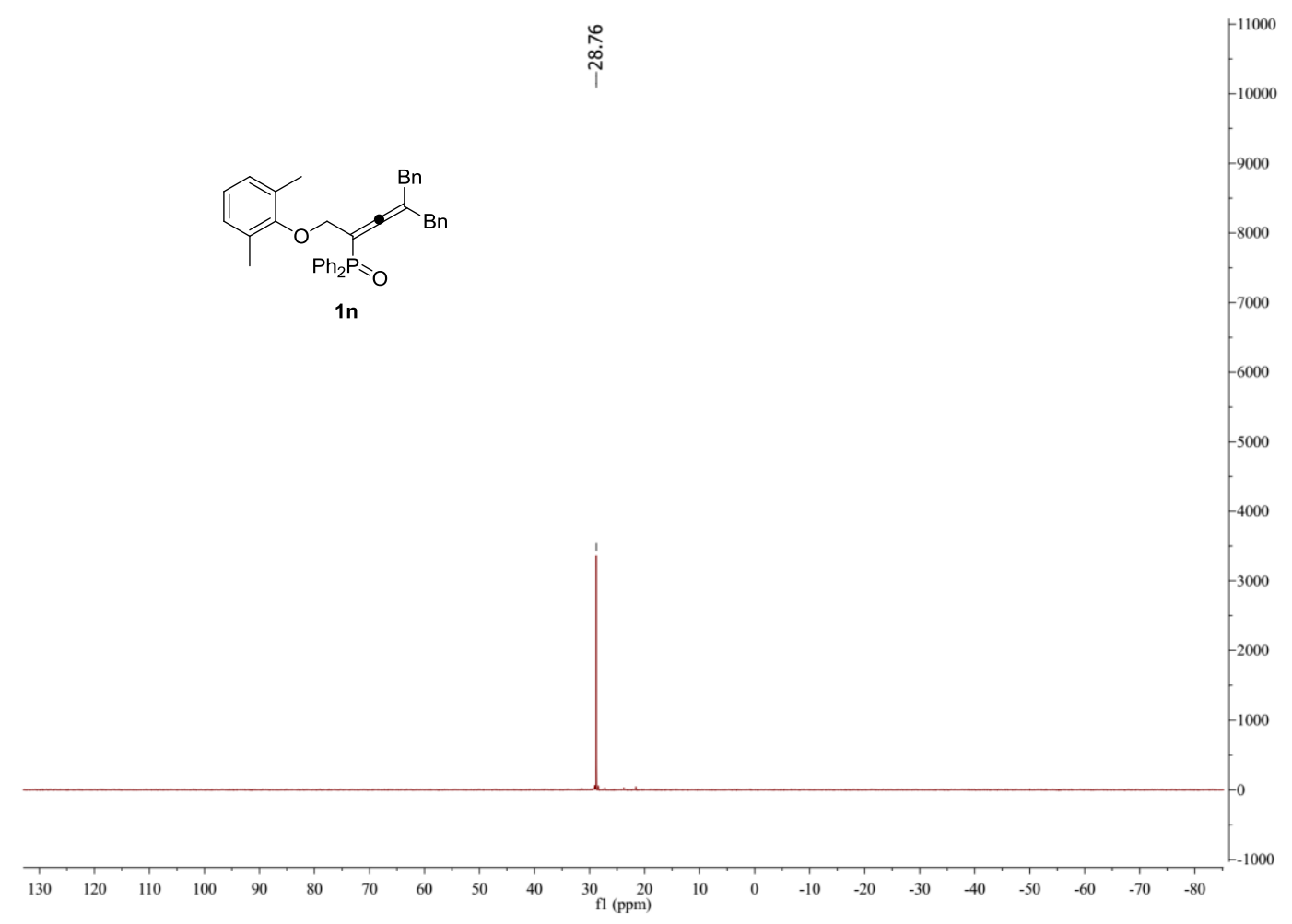

20160715-37 \#62 RT: 0.63 AV: 1 NL: 2.90E5 T: FTMS $\{1,1\}+p$ ESI Full ms [100.00-1000.00]

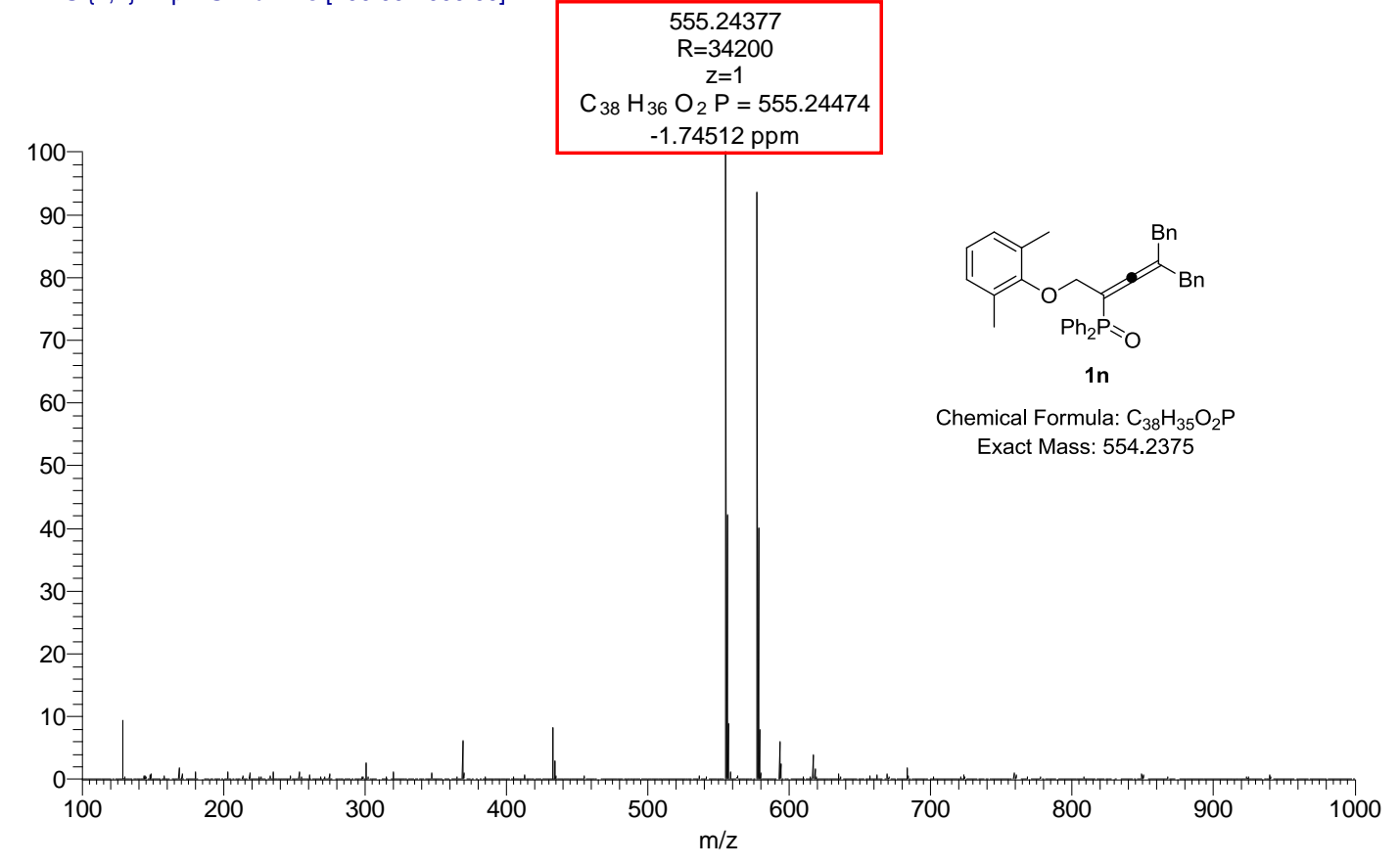




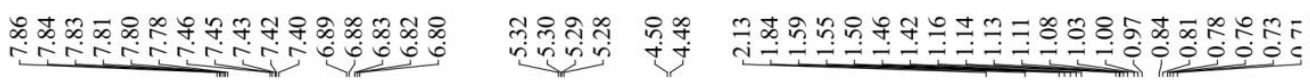

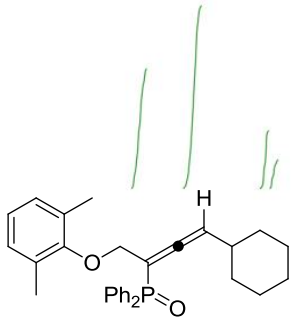

1s
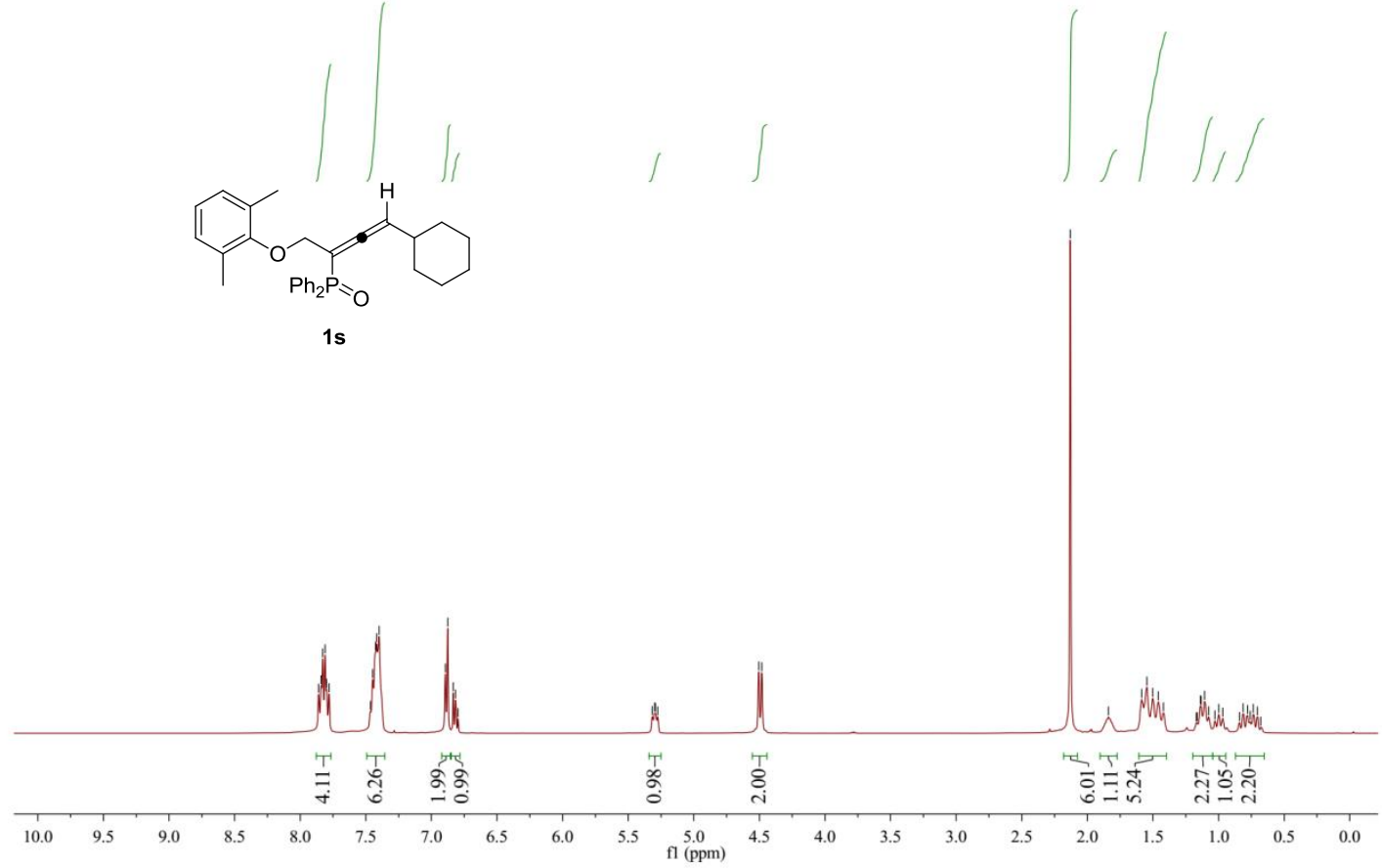

穴

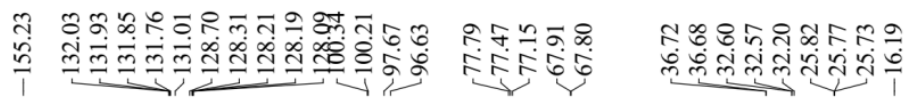

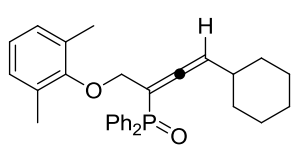

$1 \mathrm{~s}$

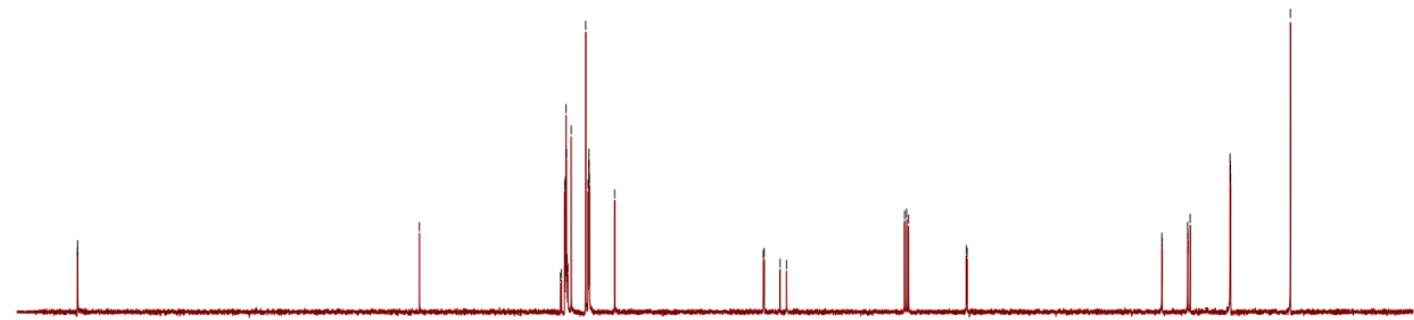

$\begin{array}{llllllllllllllllllllllllllll}210 & 200 & 190 & 180 & 170 & 160 & 150 & 140 & 130 & 120 & 110 & 100 & 90 & 80 & 70 & 60 & 50 & 40 & 30 & 20 & 10 & 0\end{array}$ 
$\bar{c}$
$\infty$
i
1

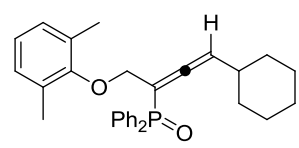

$1 \mathrm{~s}$

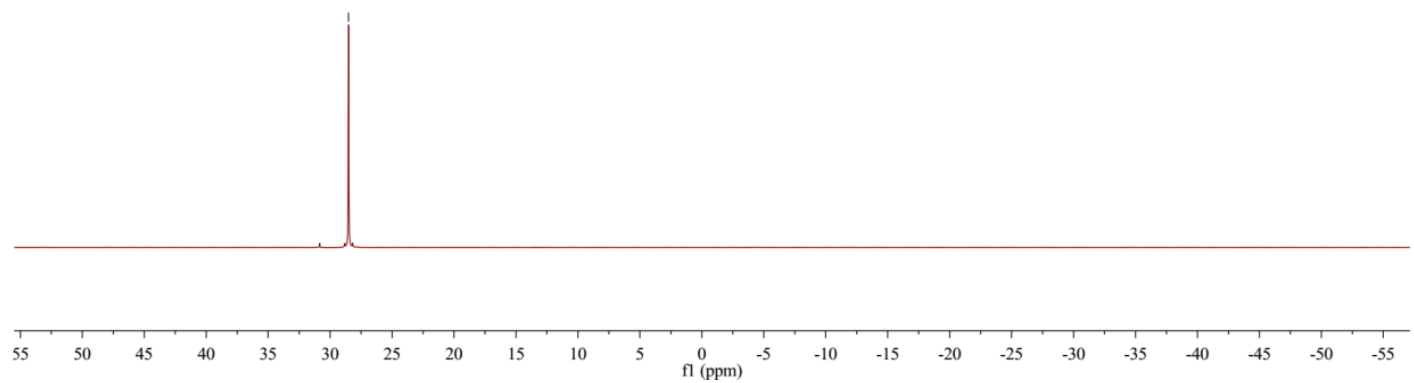

njny-20170904-20 \#71 RT: 0.62 AV: 1 SB: 33 0.01-0.03, 0.70-1.03 NL: 4.73E5 T: FTMS $\{1,1\}+p$ ESI Full ms [100.00-1000.00]

479.21077
$\mathrm{R}=36662$

$\mathrm{C}_{30} \mathrm{H}_{33} \mathrm{O}_{2} \mathrm{NaP}=479.21104$

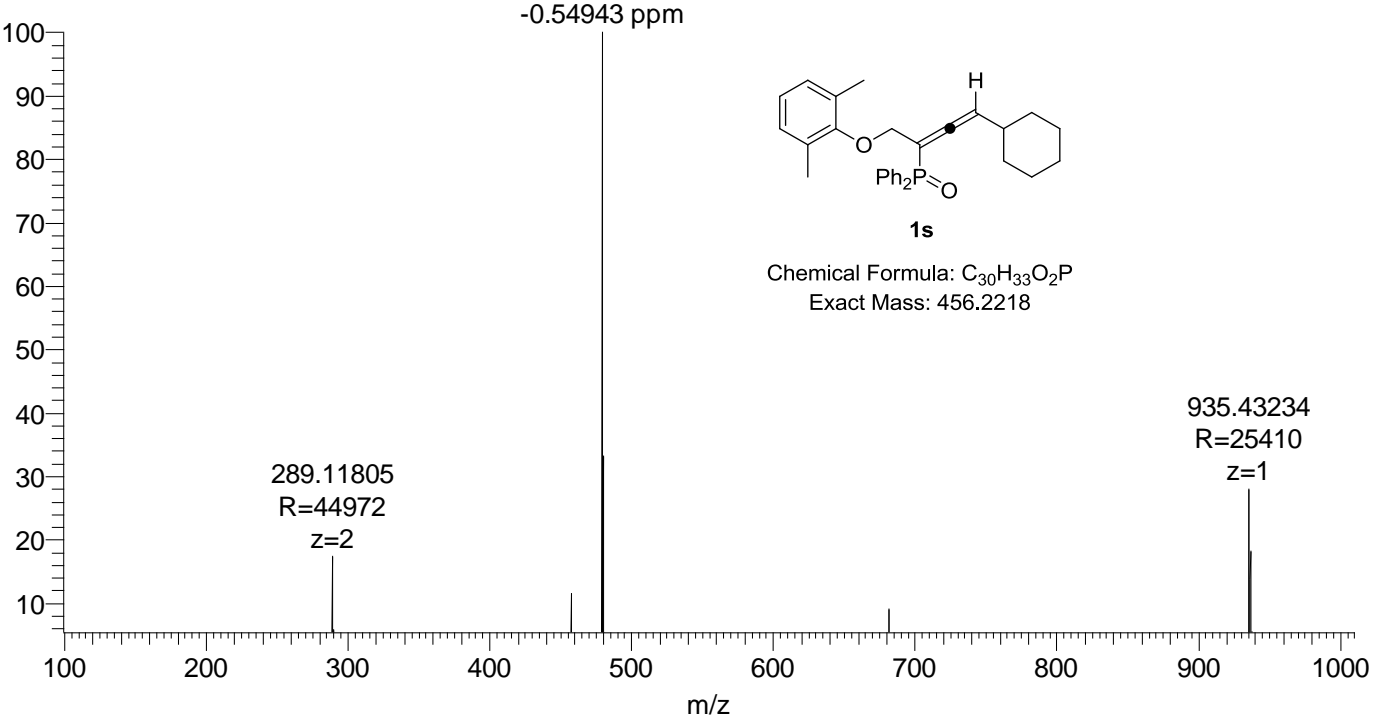




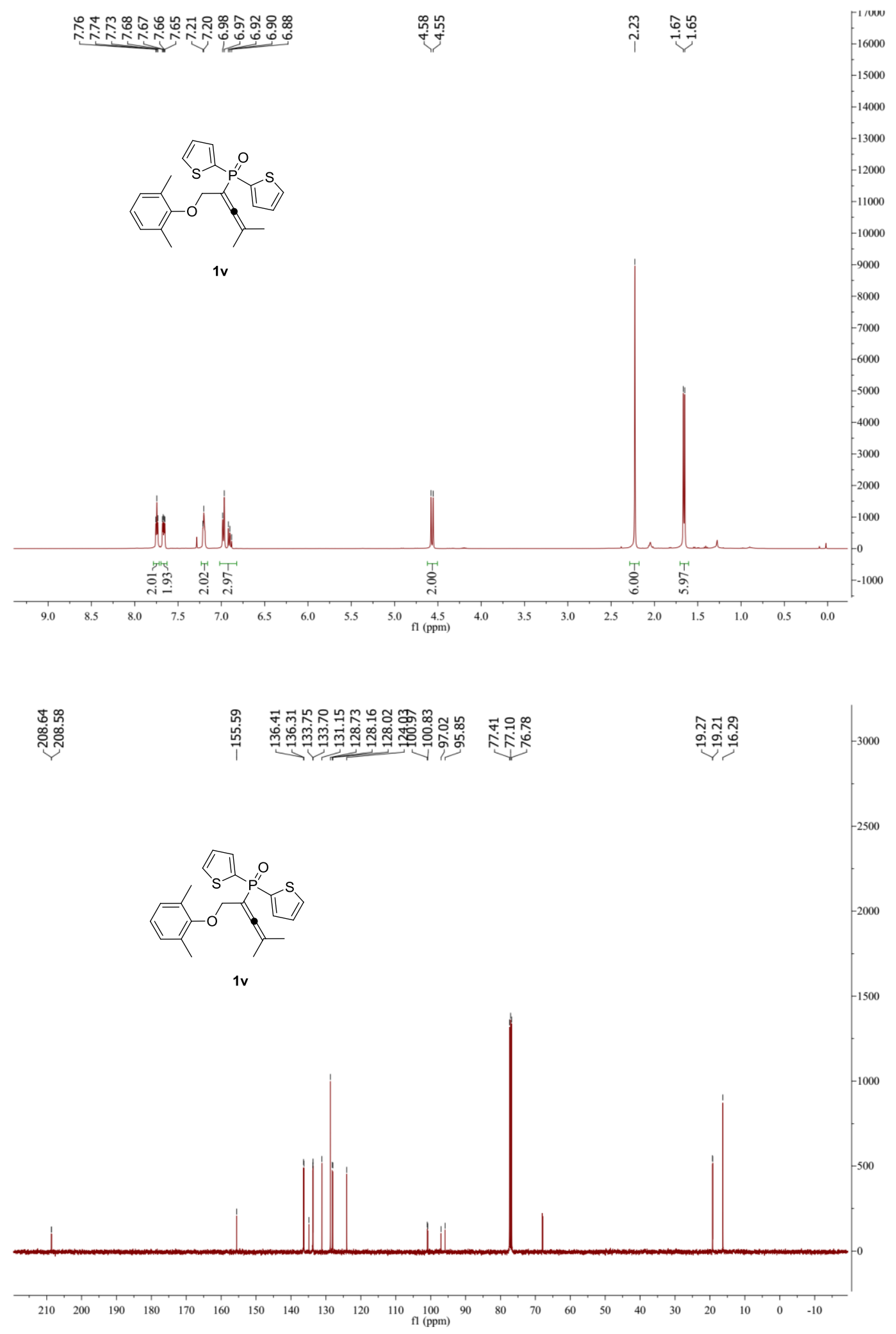




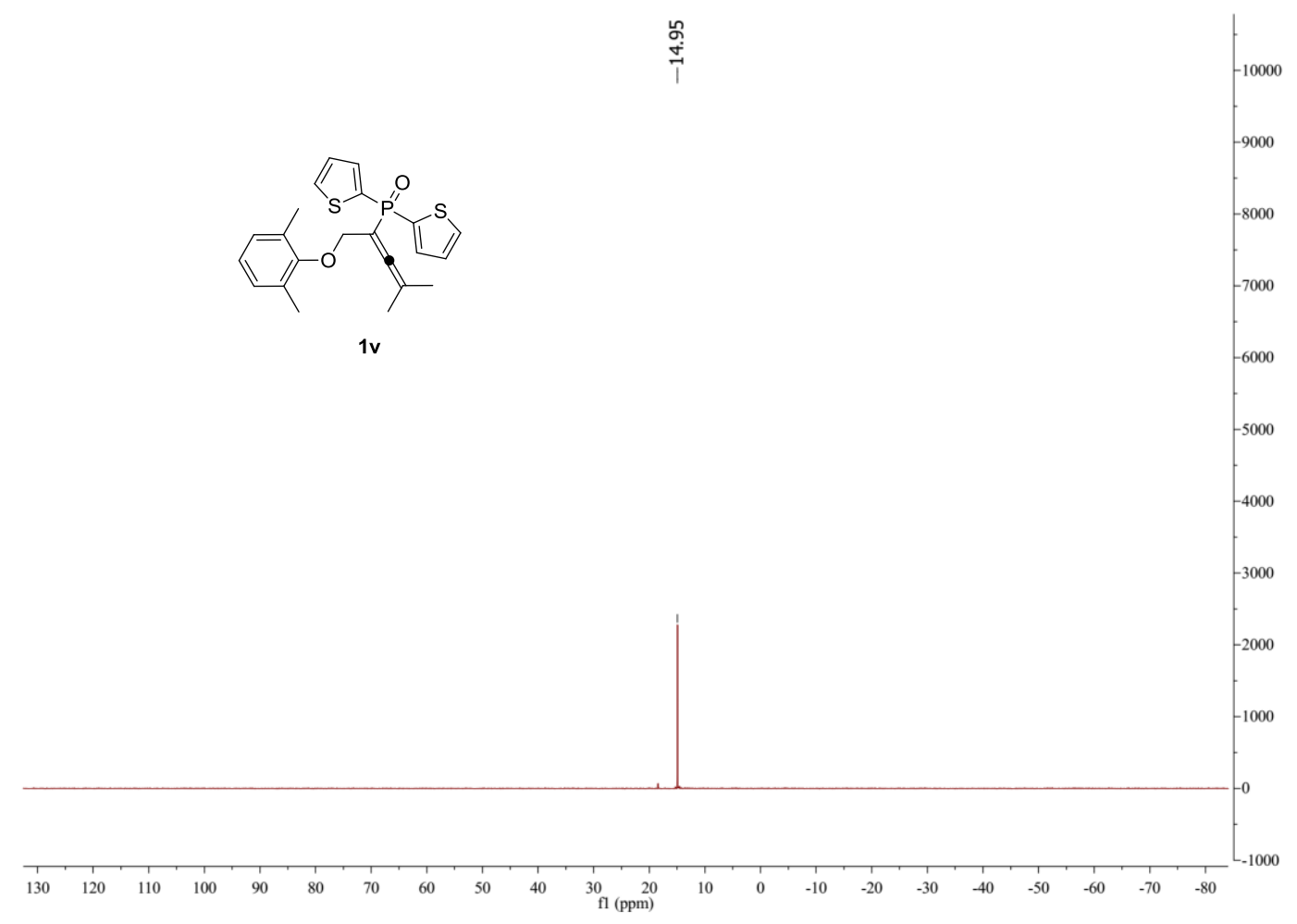

NJNY-3_180305125425 \#29 RT: 0.43 AV: 1 NL: $1.21 E 6$

T: FTMS $\{1,1\}+p$ ESI Full ms [100.00-1000.00] 437.07678
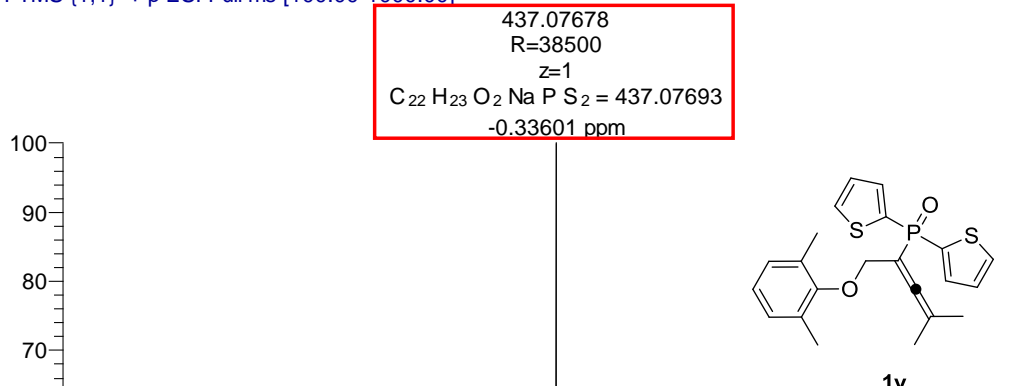

$1 v$

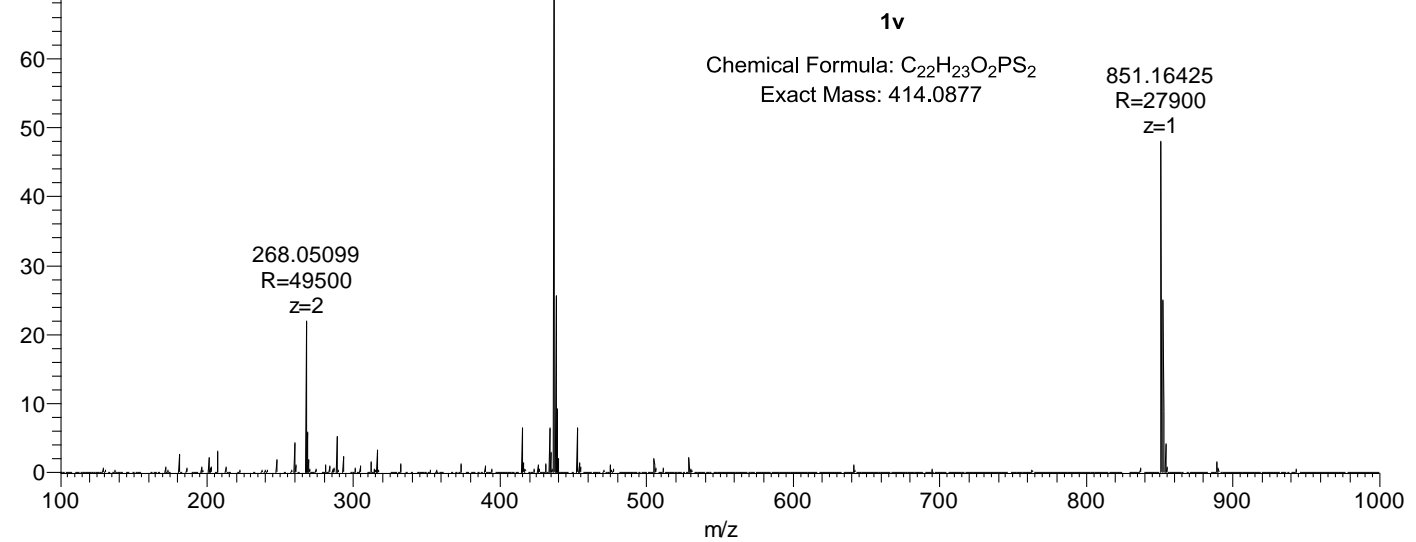



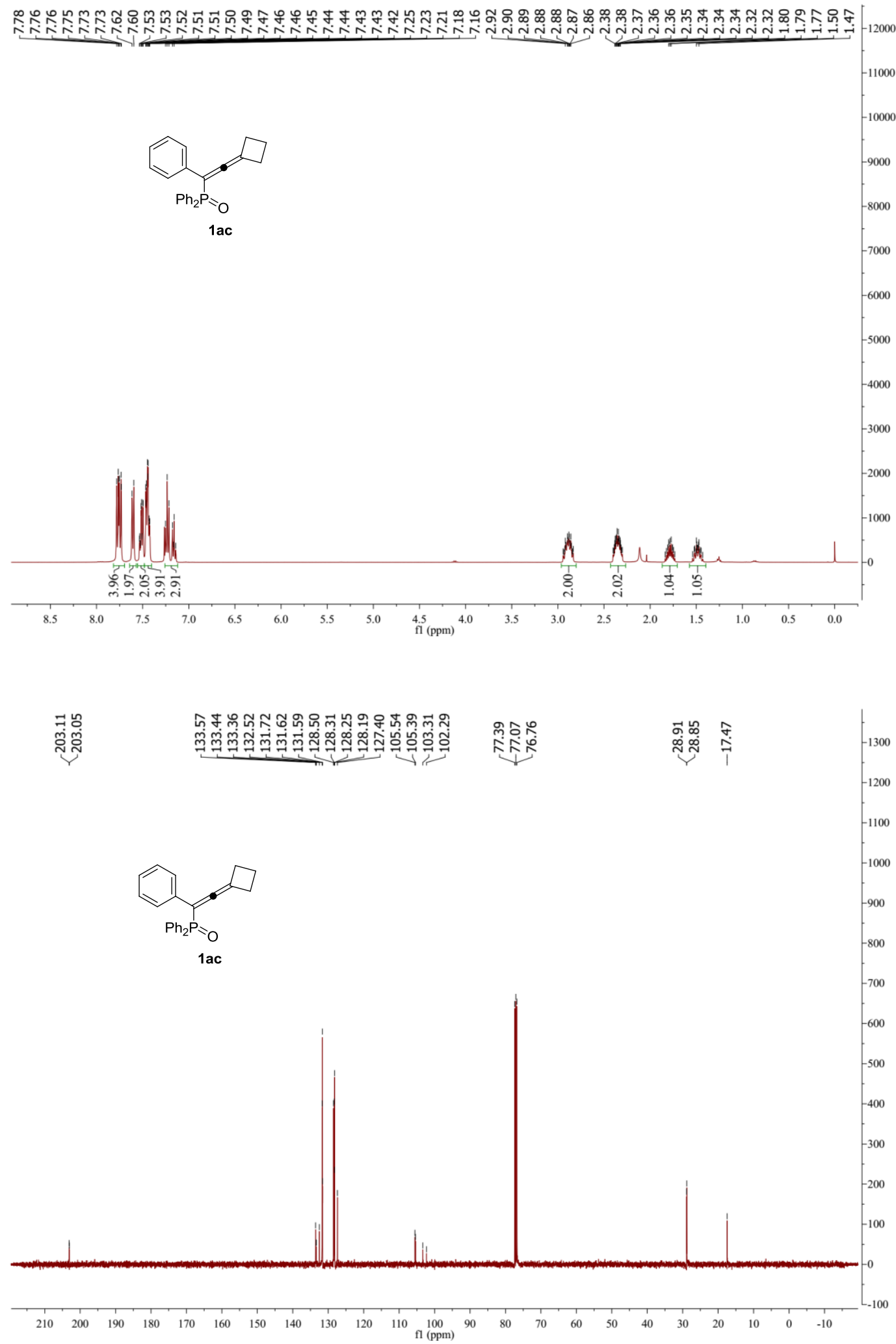


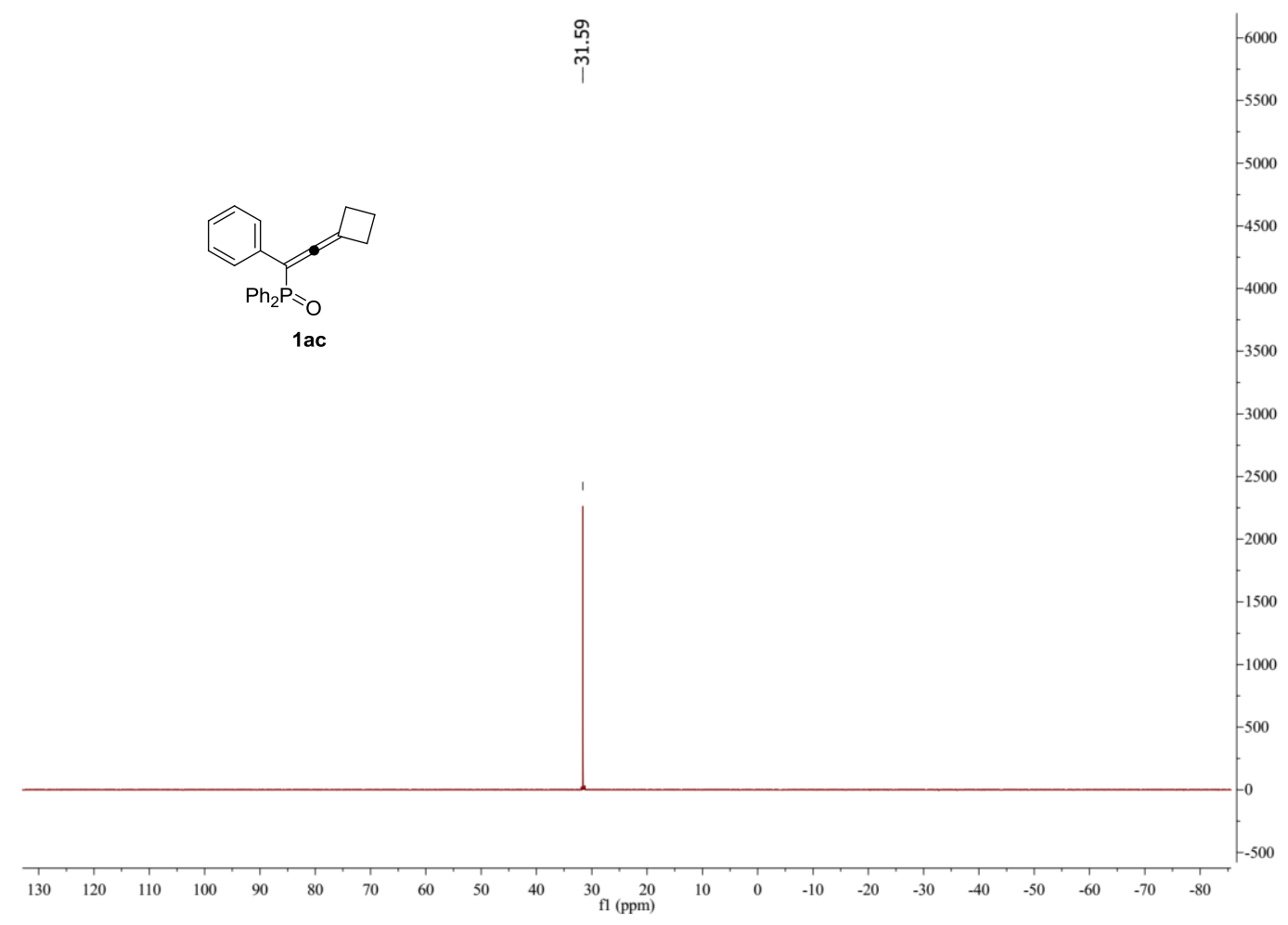

35 \#49 RT: 0.67 AV: 1 NL: $3.76 E 7$

T: FTMS $\{1,1\}+$ p ESI Full ms [100.00-1000.00]

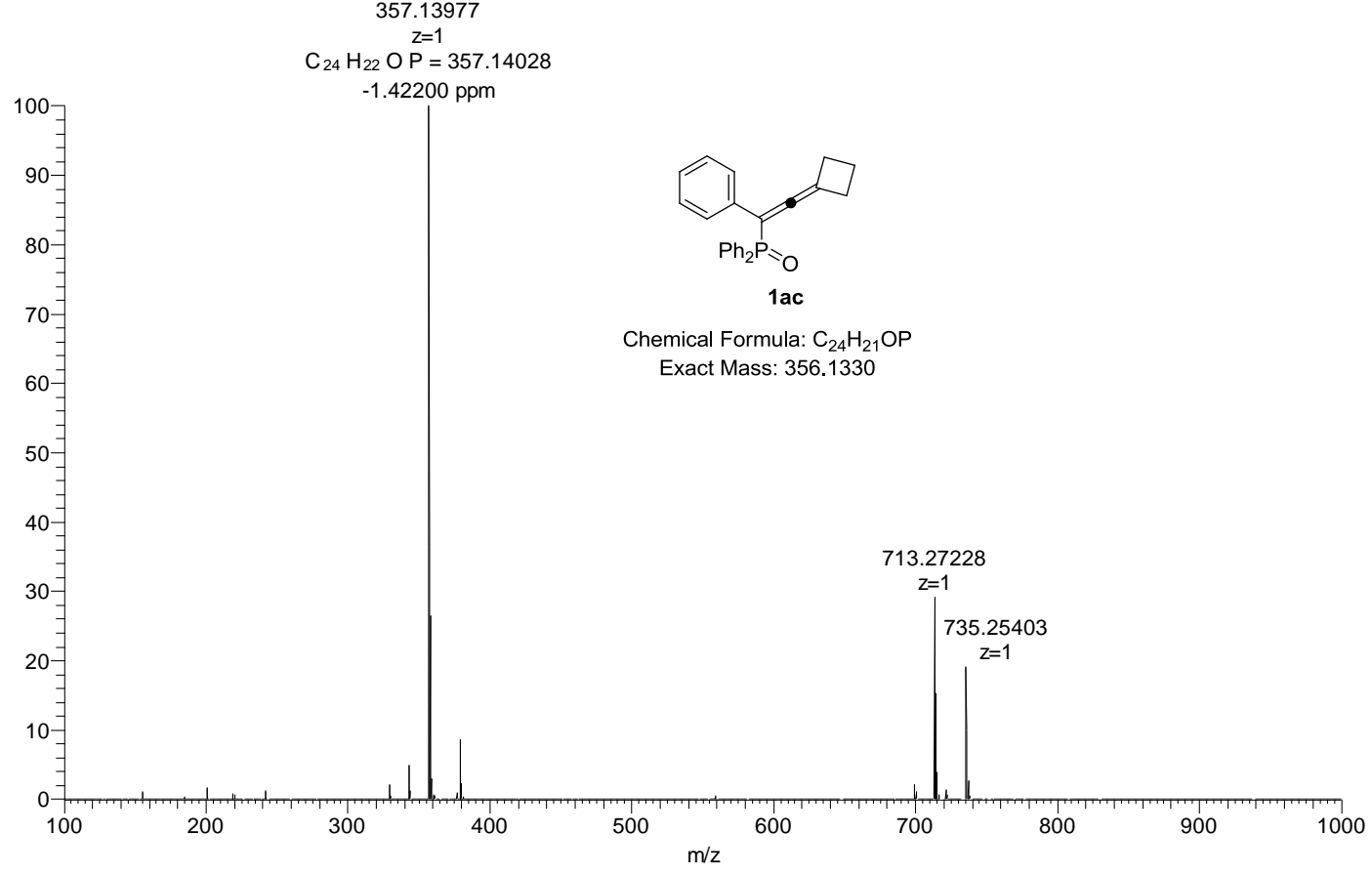



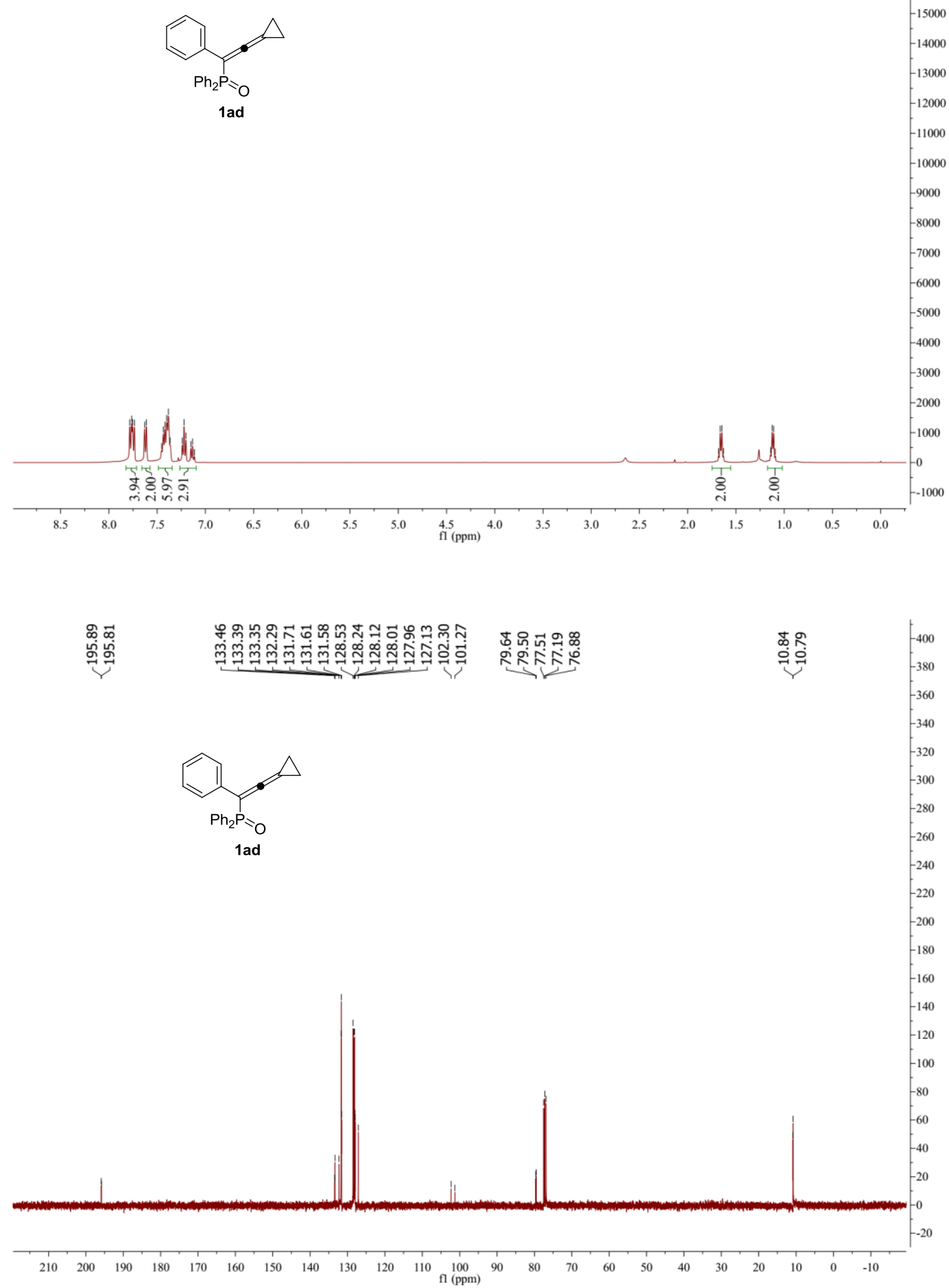


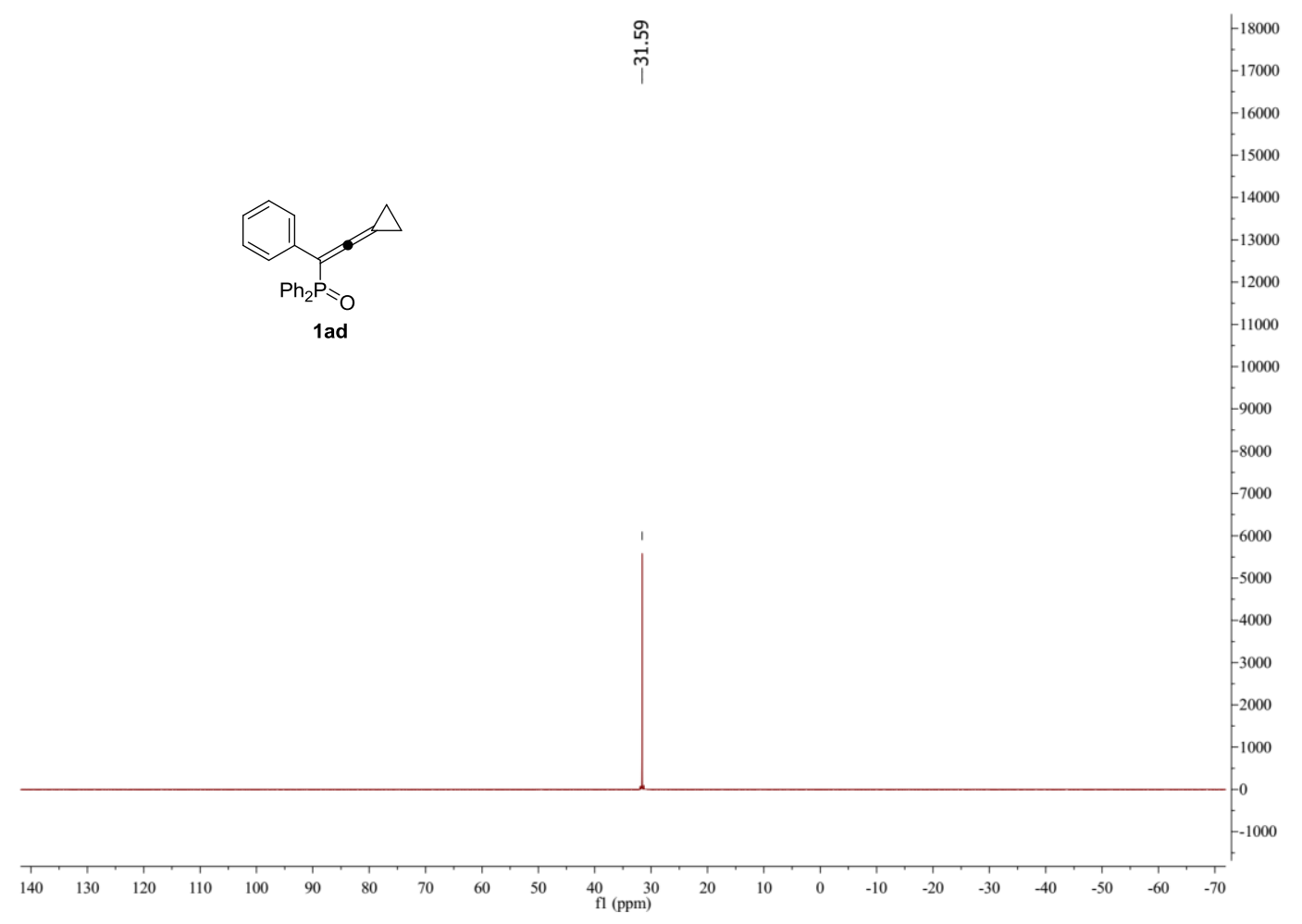

33 \#51 RT: 0.71 AV: 1 NL: $3.48 E 7$

T: FTMS $\{1,1\}+p$ ESI Full ms [100.00-1000.00]

$$
\begin{gathered}
343.12415 \\
\mathrm{Z}=\text { ? }
\end{gathered}
$$

$\mathrm{C}_{23} \mathrm{H}_{20} \mathrm{O} \mathrm{P}=343.12463$

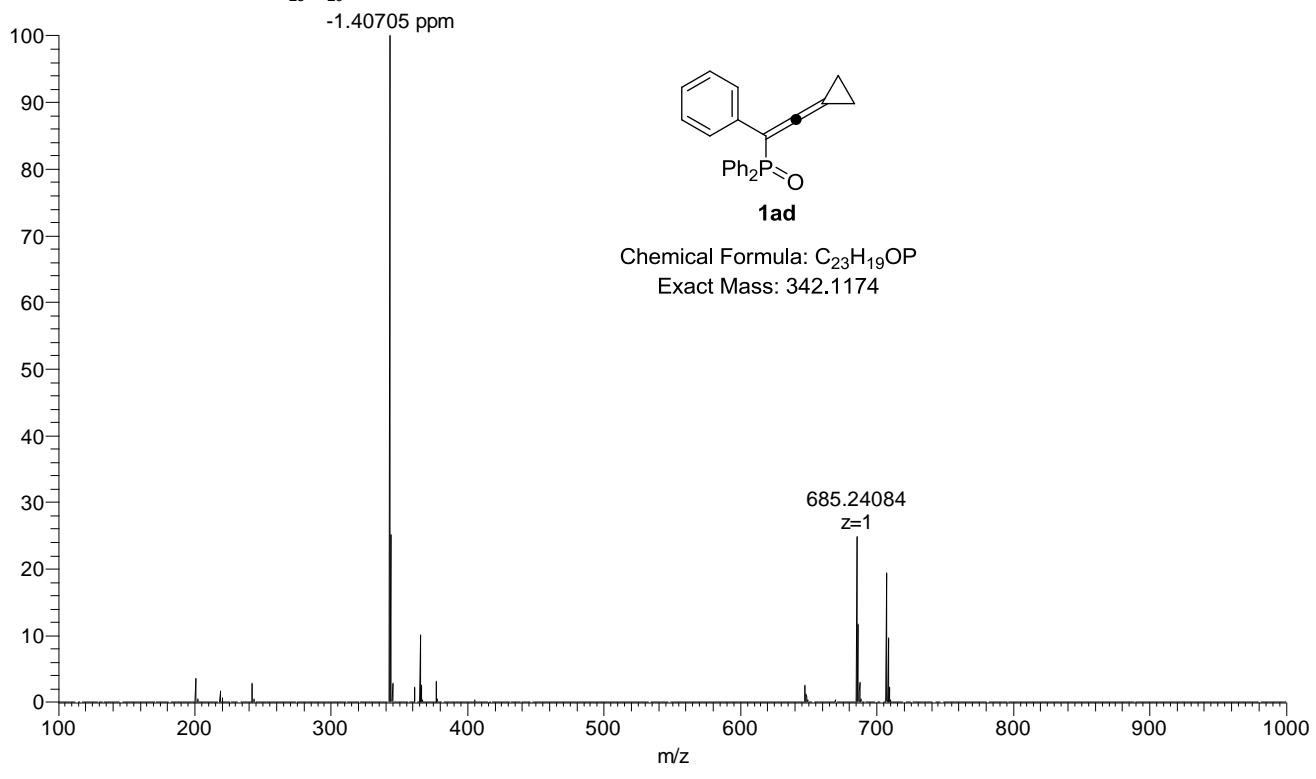




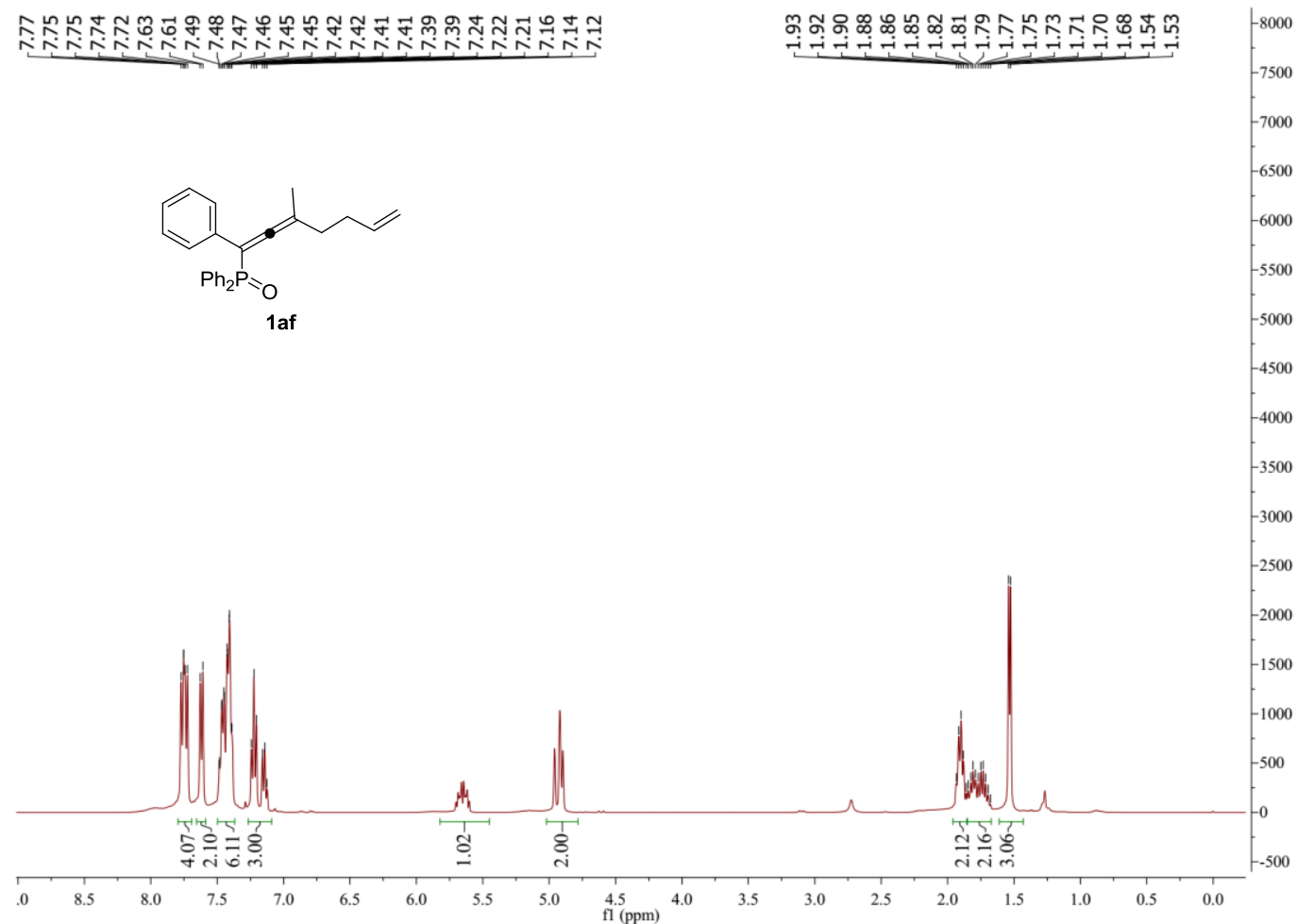

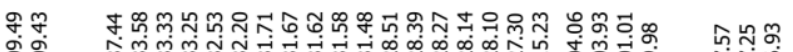

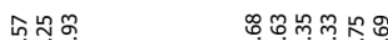

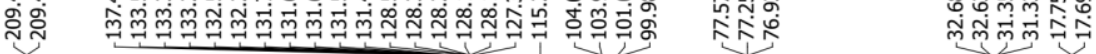
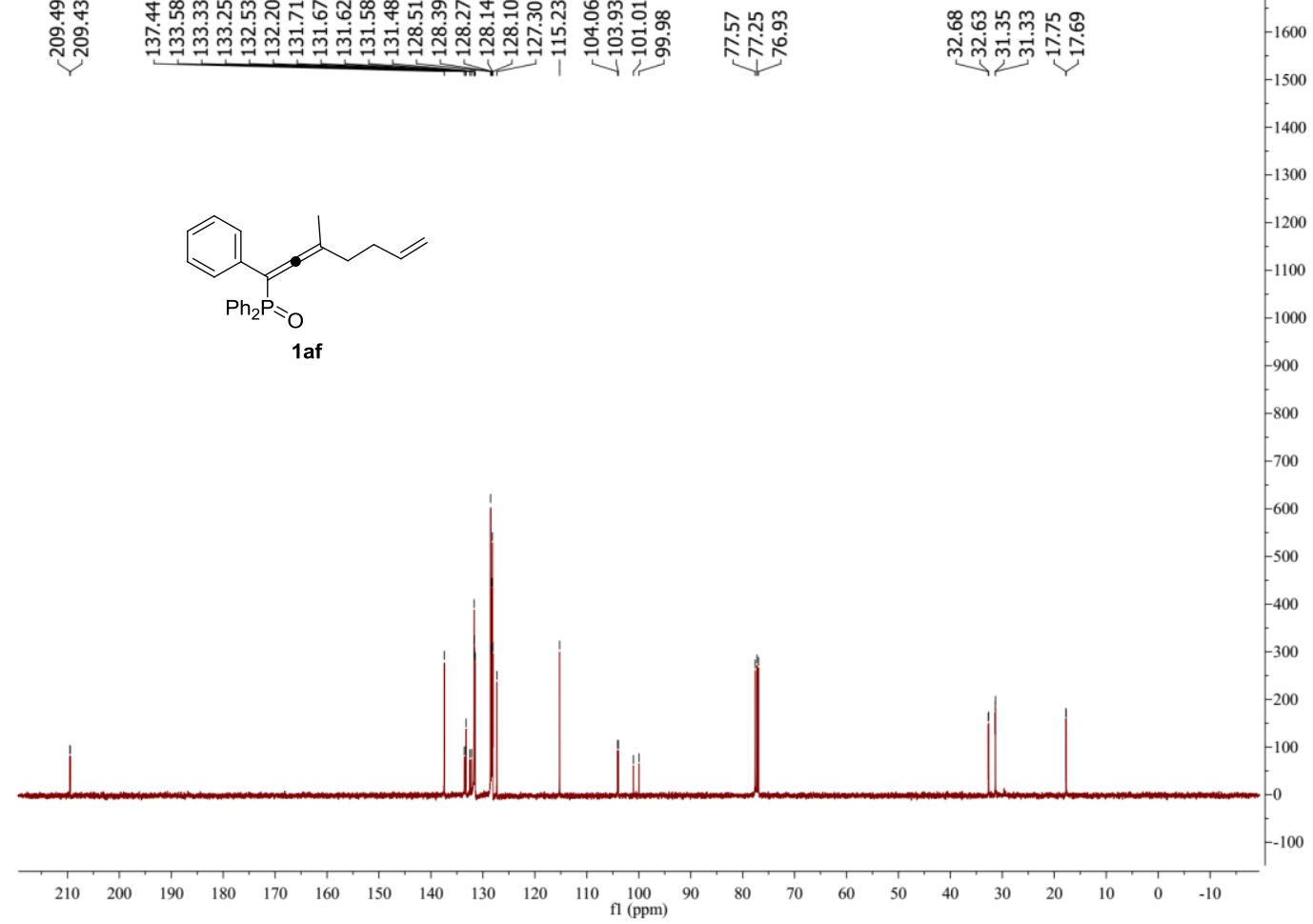


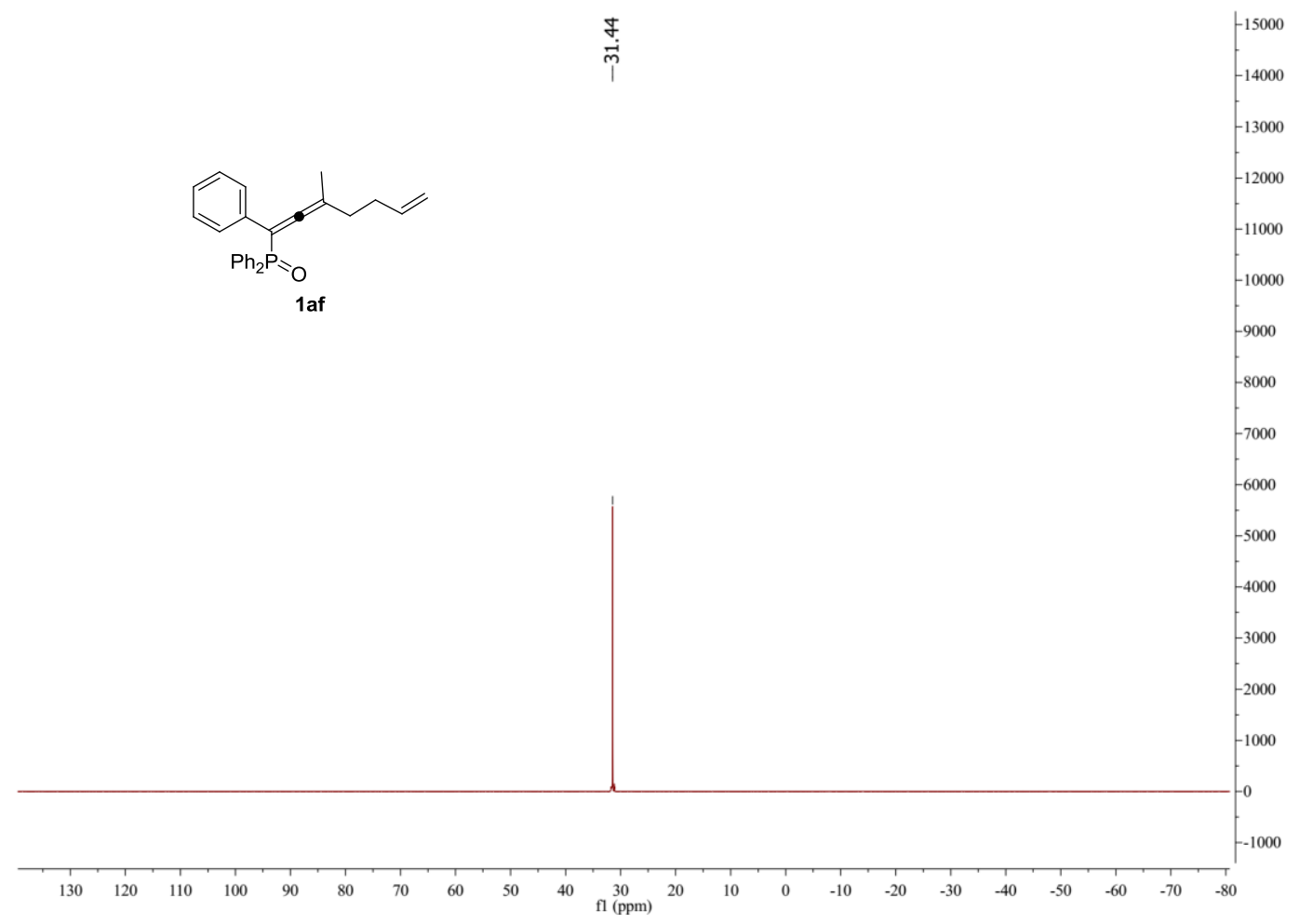

njny-20180521-3 \#65 RT: 0.96 AV: 1 NL: 2.58E6

T: FTMS $\{1,1\}+p$ ESI Full ms [100.00-1000.00]

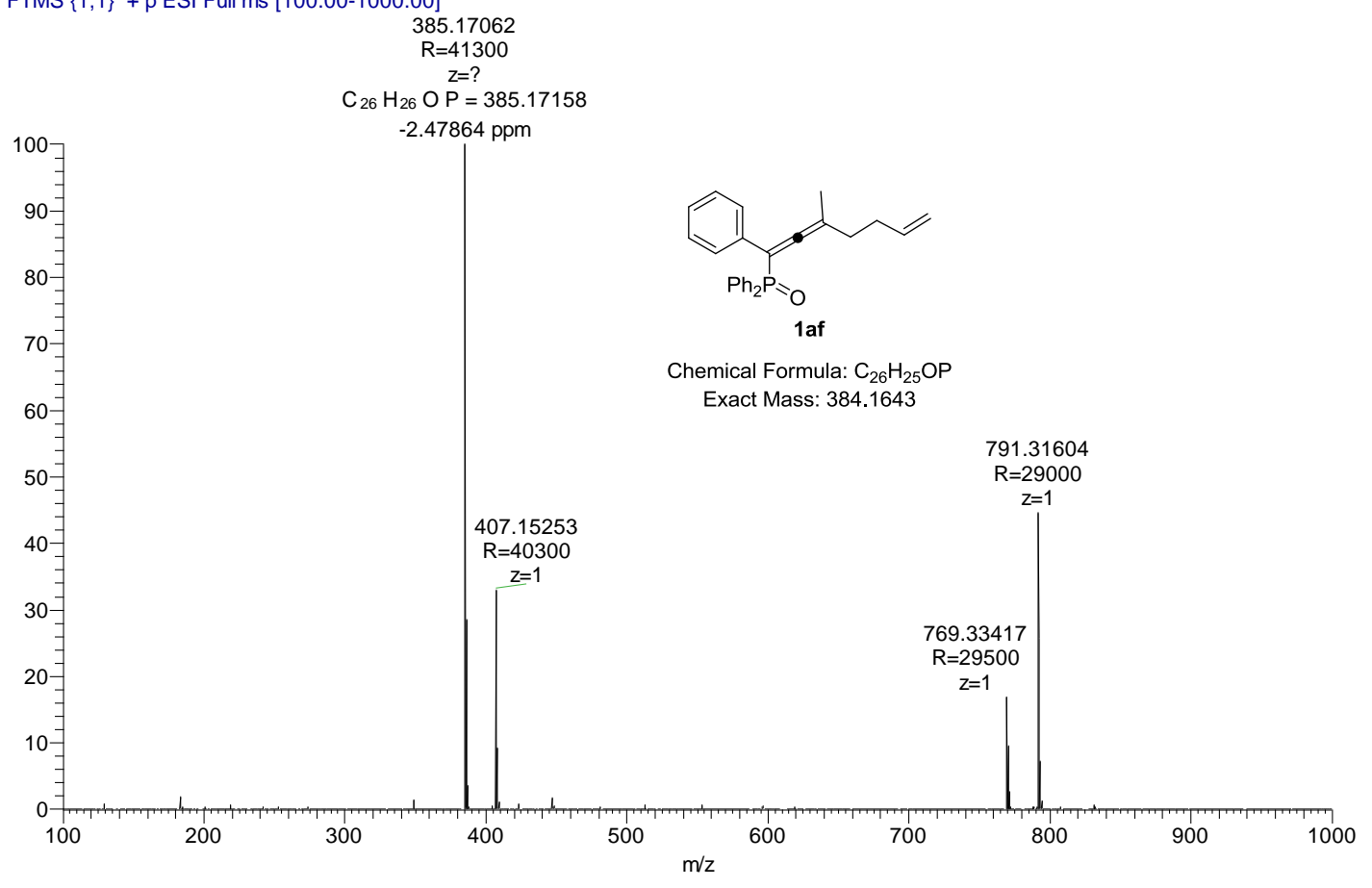



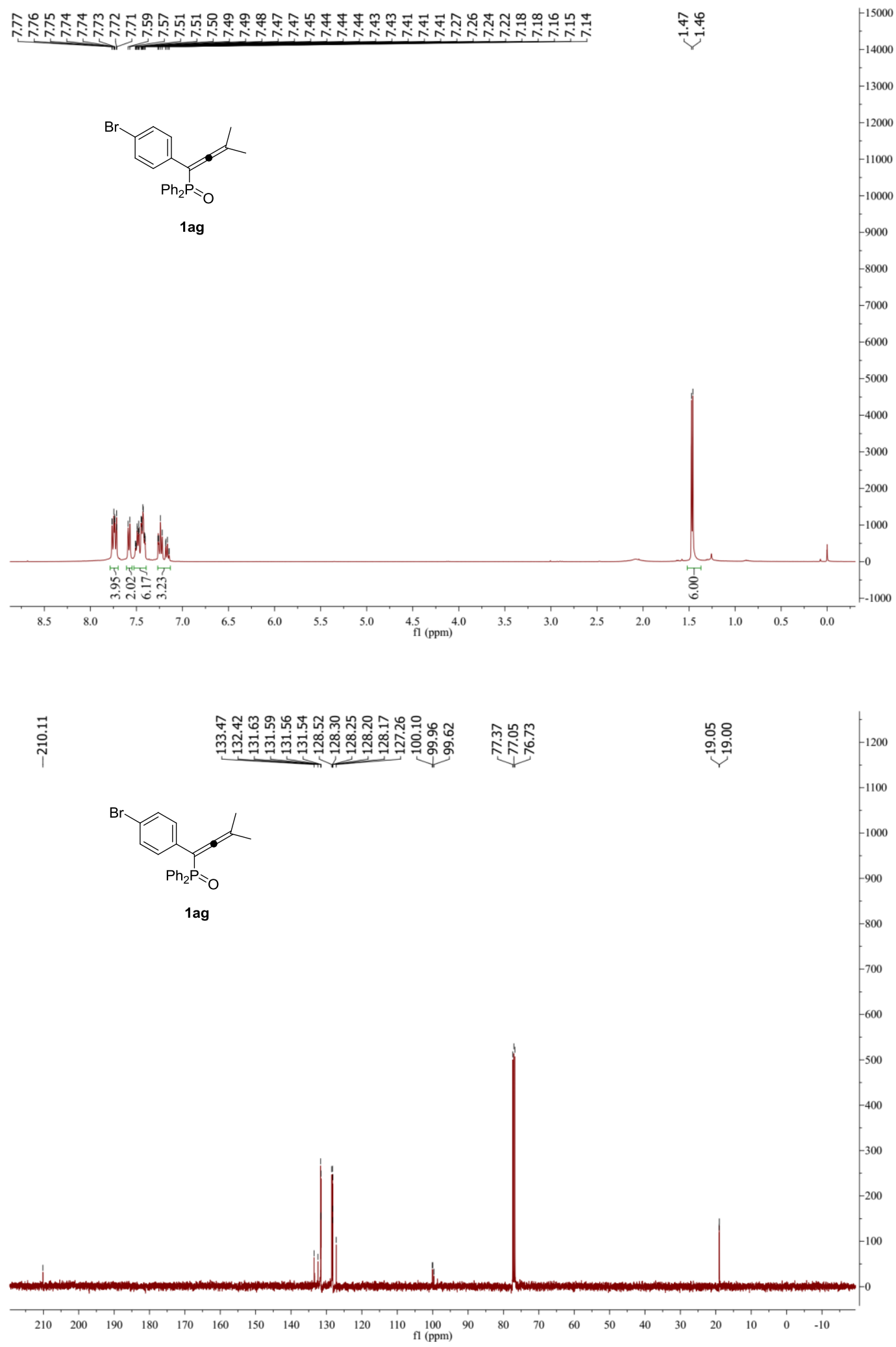


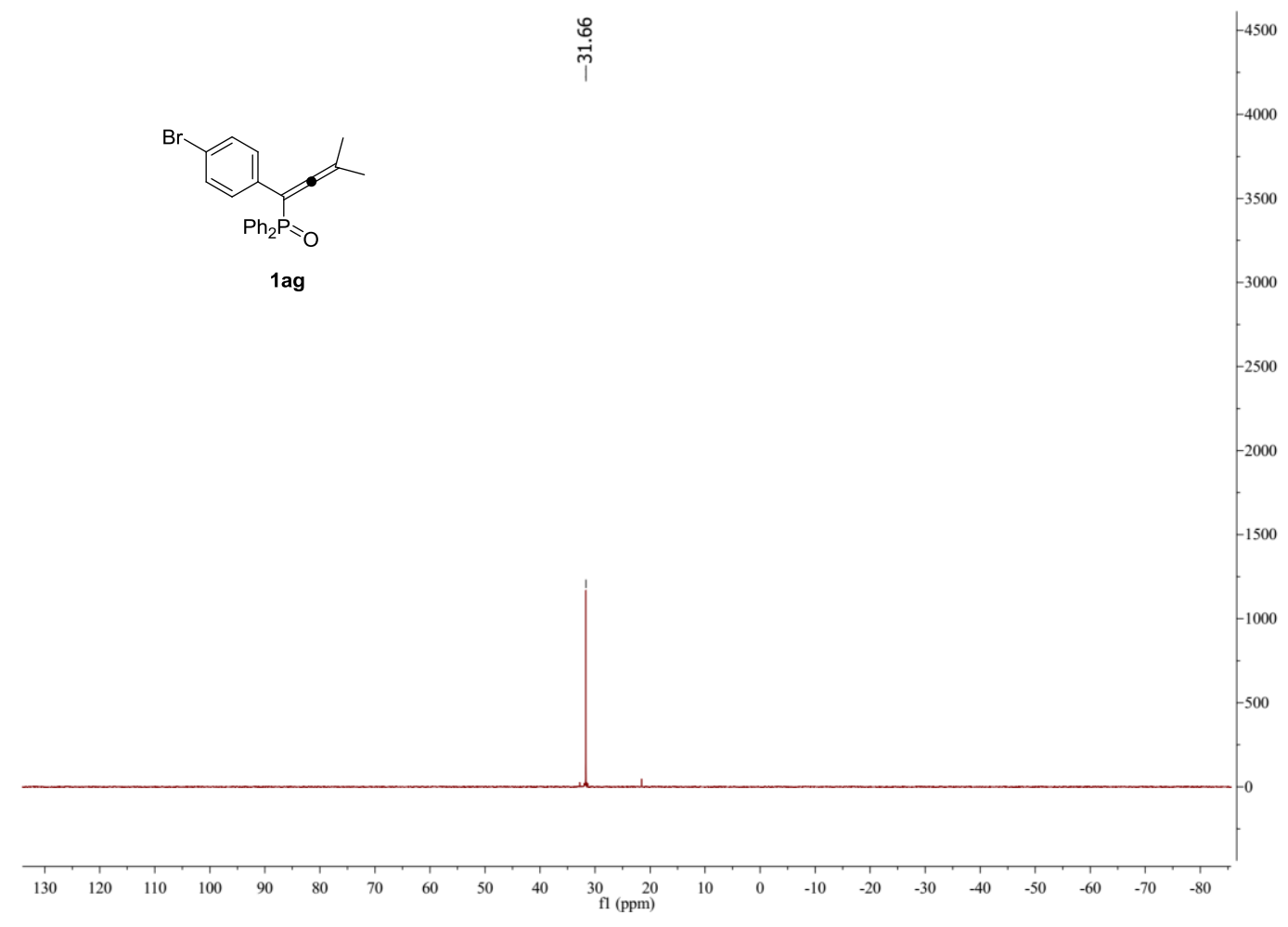

njny-20180521-4 \#55 RT: 0.84 AV: 1 NL: 8.06E3

T: FTMS $\{1,1\}$ + p ESI Full ms [100.00-1000.00]
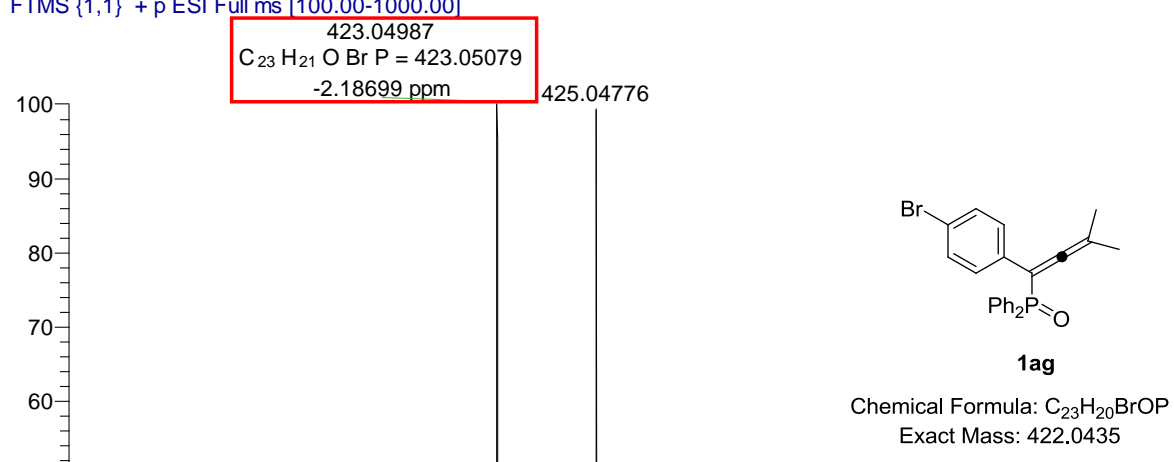

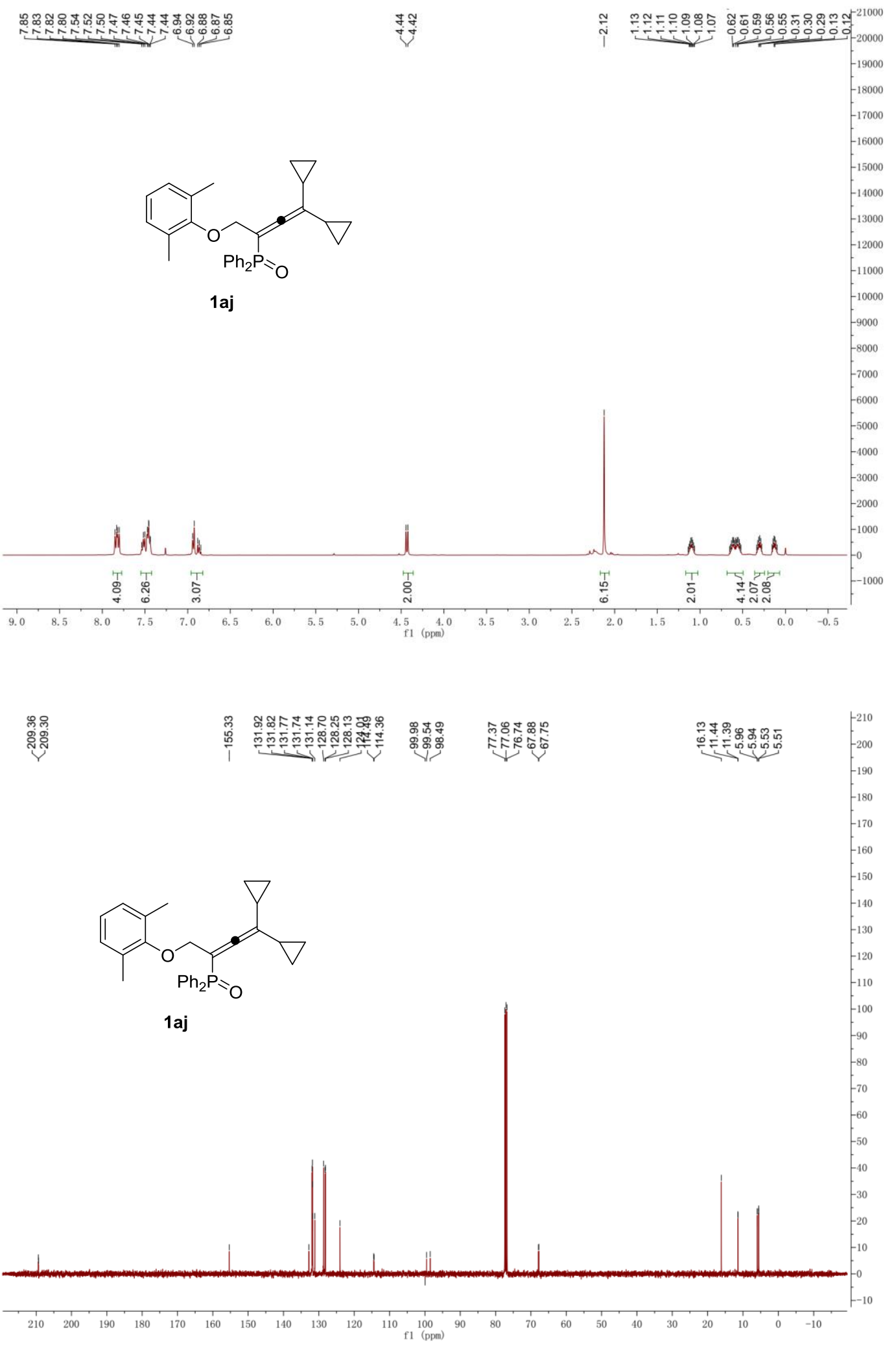


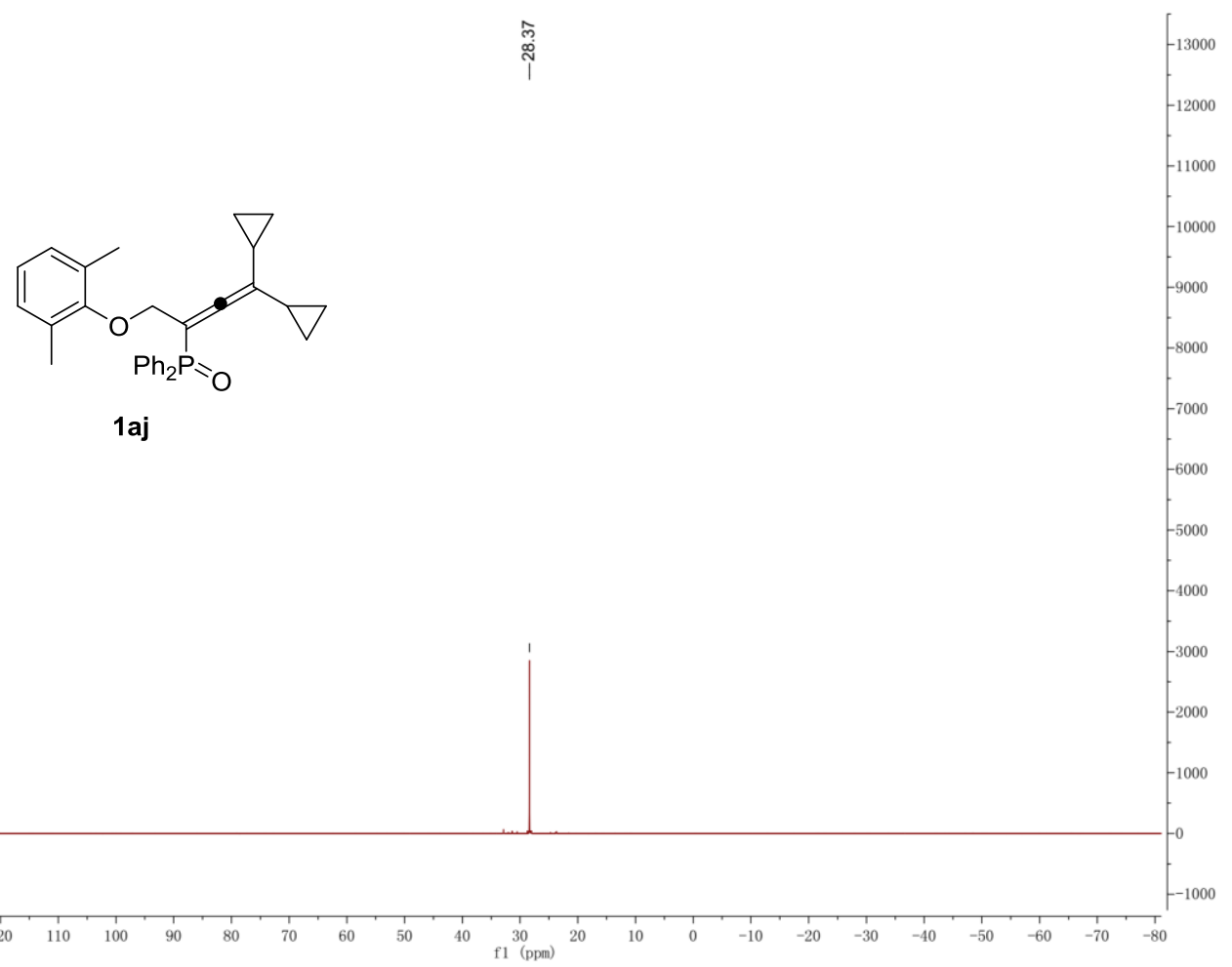

20160715-38 \#55 RT: 0.54 AV: 1 NL: 2.00E5 T: FTMS $\{1,1\}+p$ ESI Full ms [100.00-1000.00]

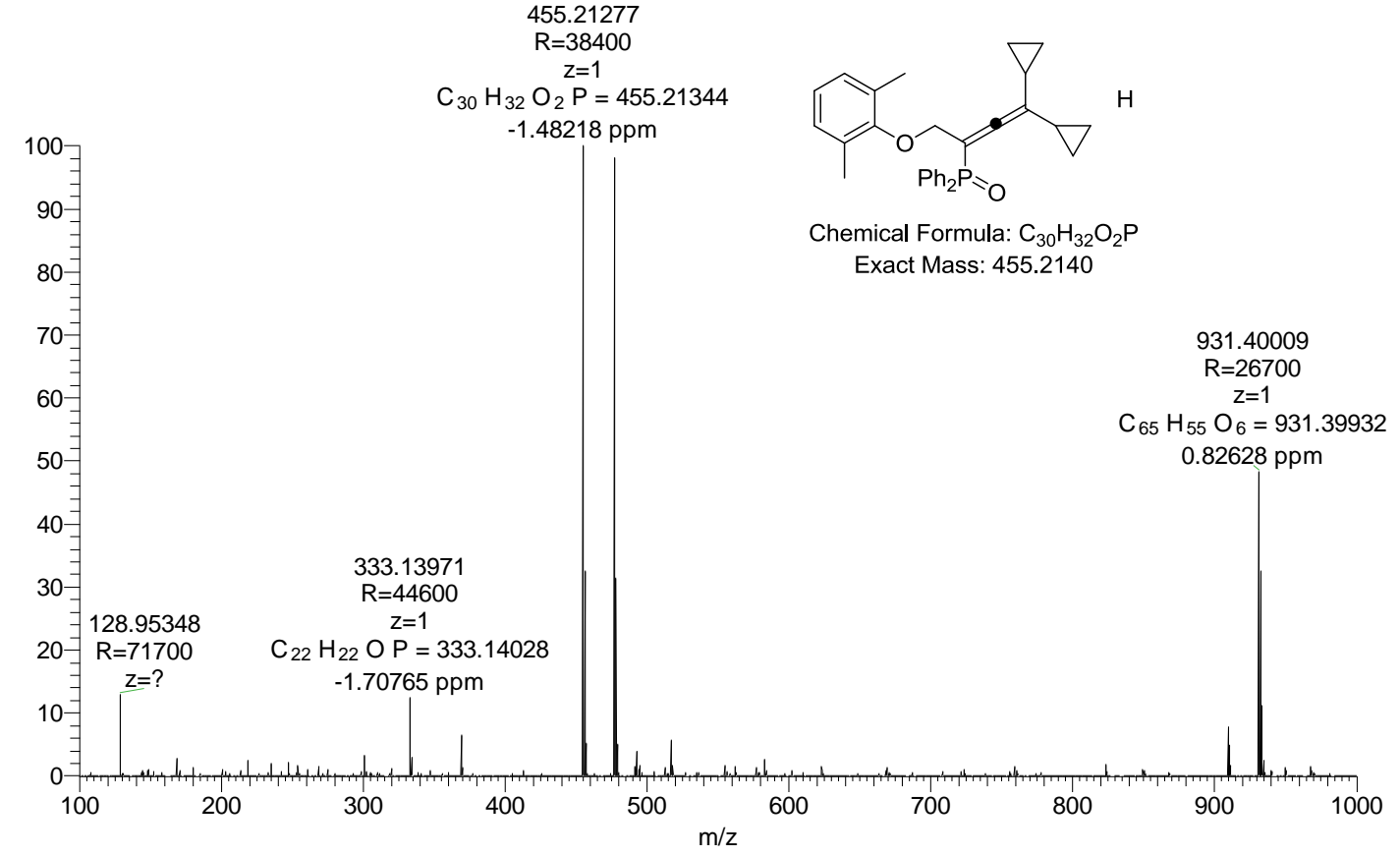



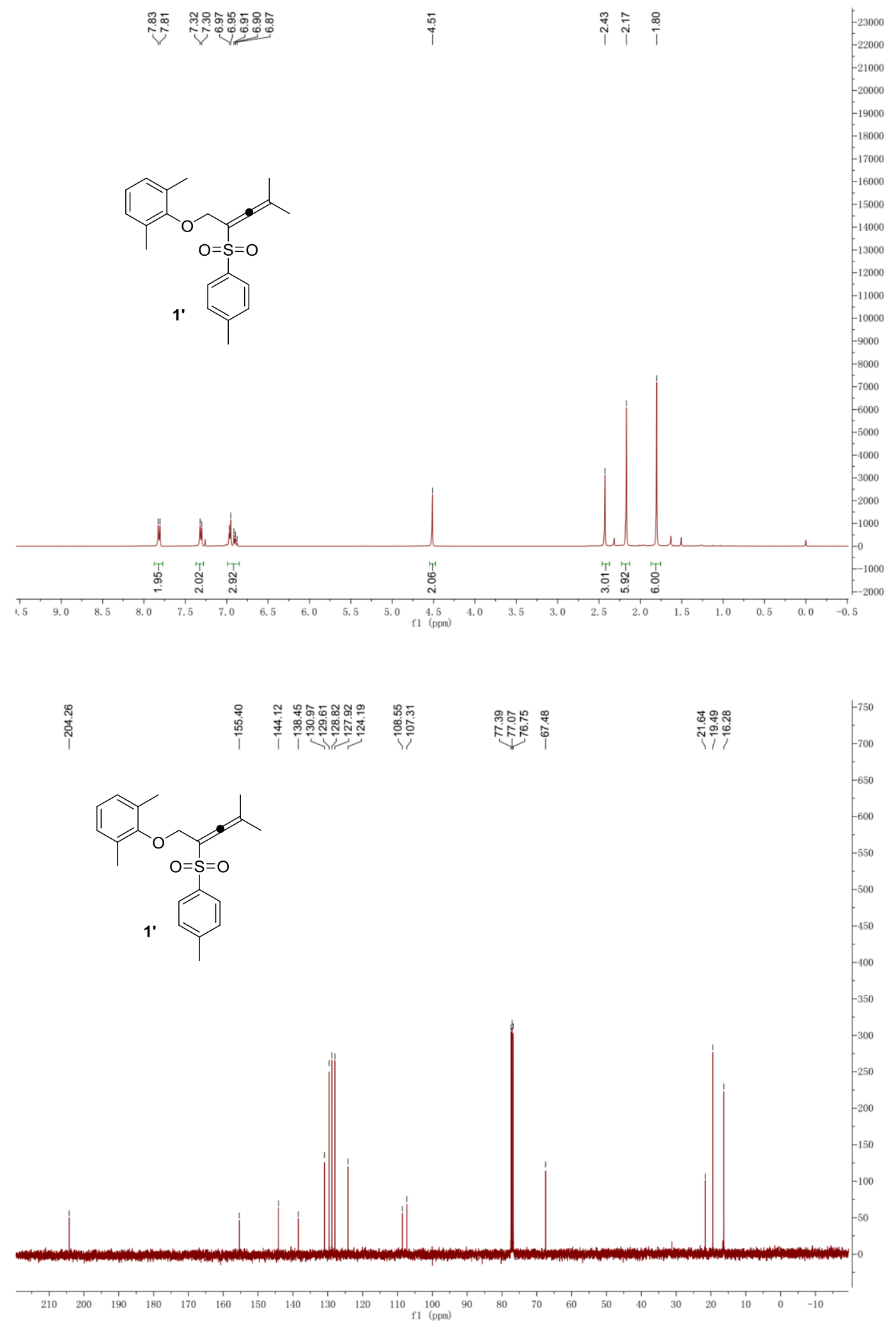
20170618-13 \#51-52 RT: 0.46-0.47 AV: 2 NL: 5.73E5

T: FTMS $\{1,1\}+p$ ESI Full ms [100.00-1000.00]
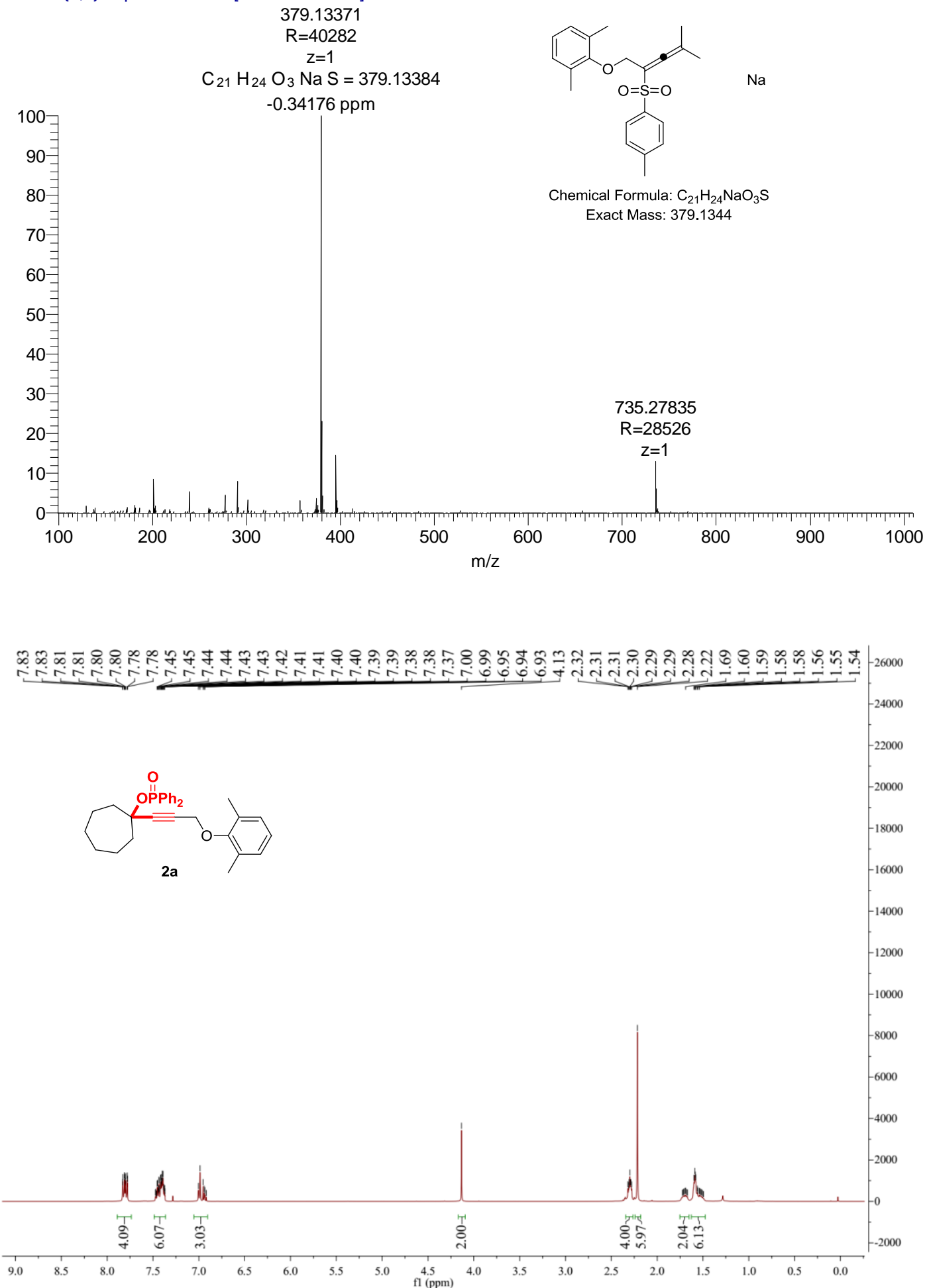

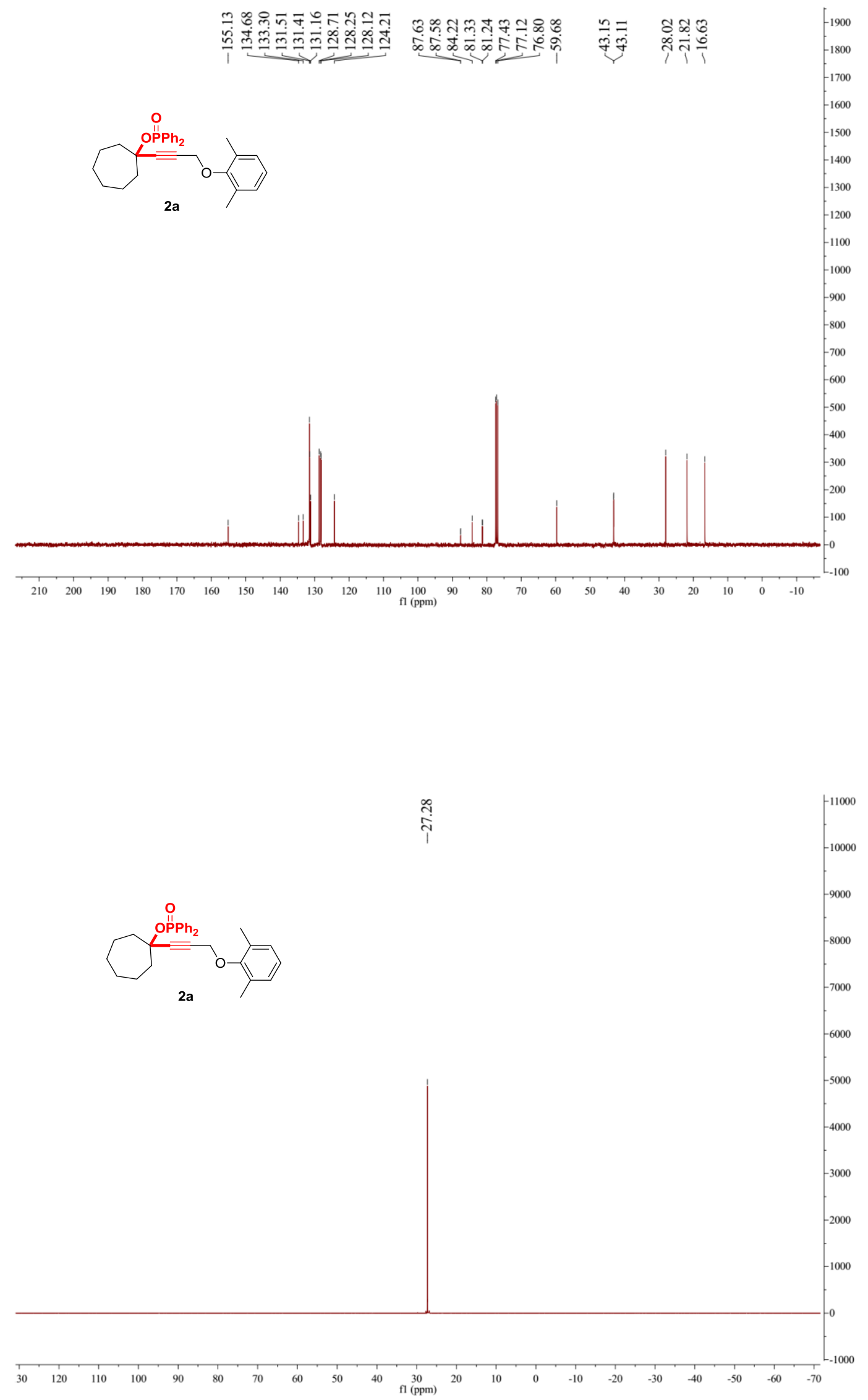
20171124-18 \#41-42 RT: 0.36-0.37 AV: 2 NL: $2.82 E 6$ T: FTMS $\{1,1\}+p$ ESI Full ms [100.00-1000.00]
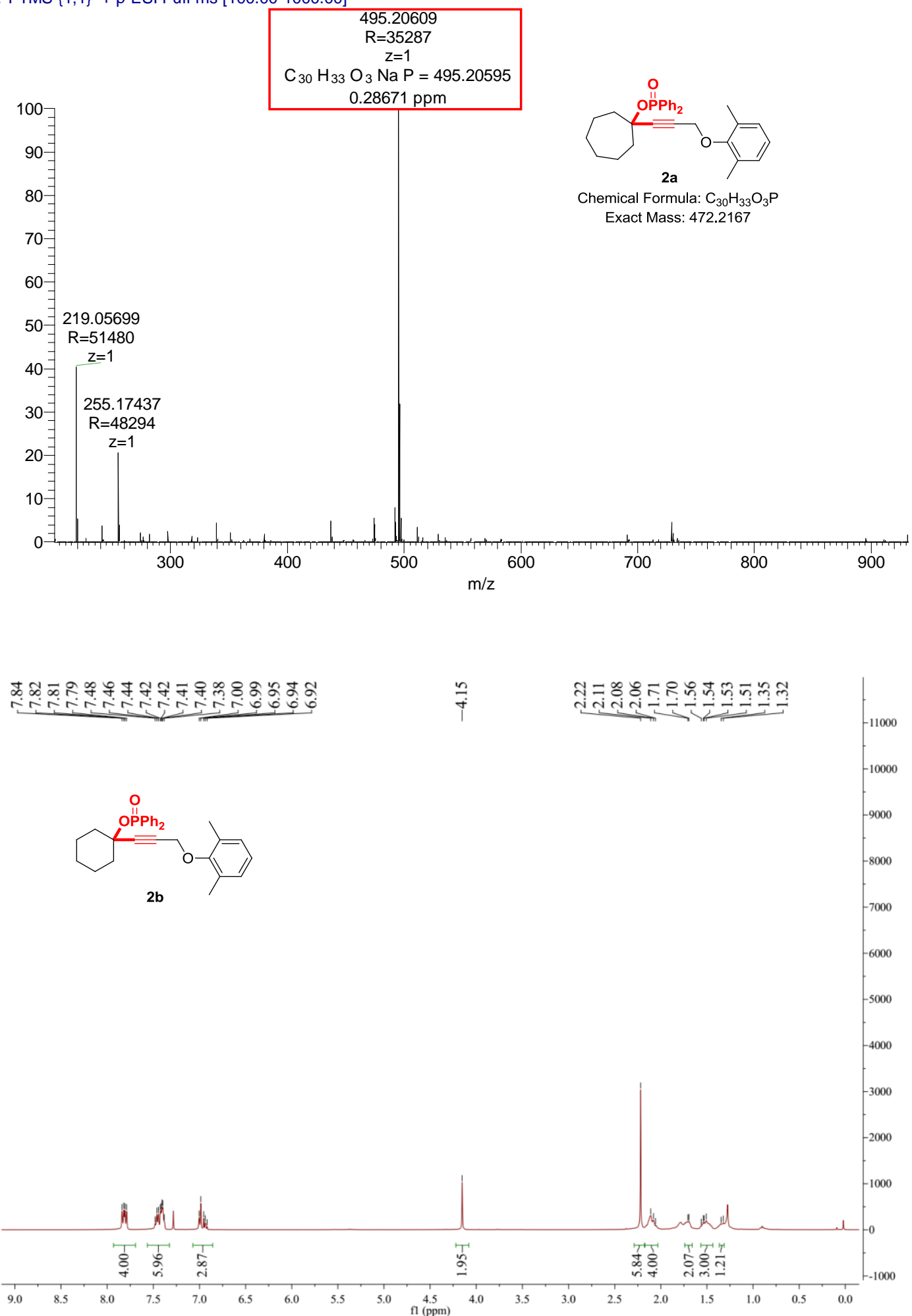


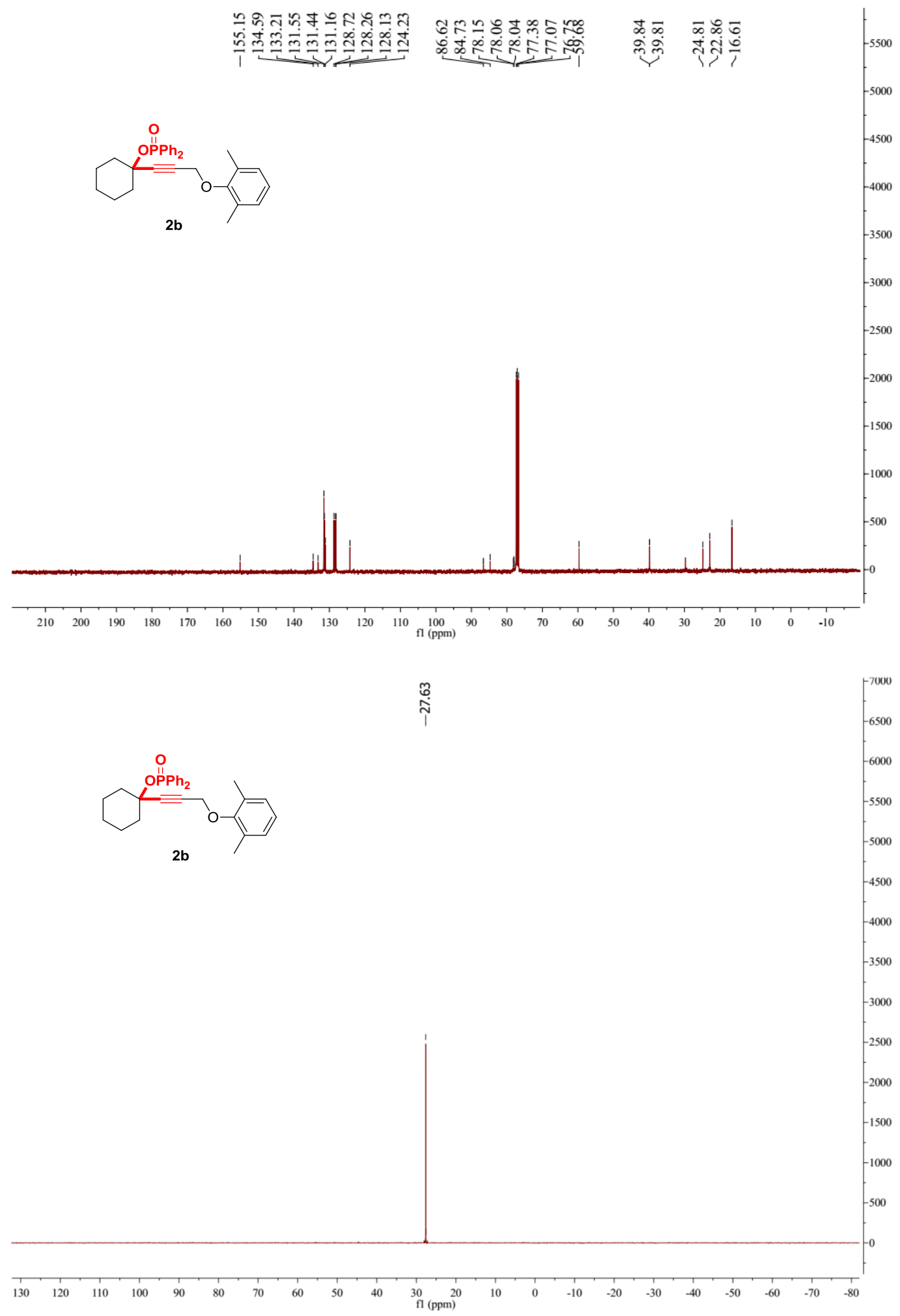


20170505-49_170506165143 \#32-36 RT: 0.27-0.31 AV: 5 NL: 5.56E5

T: FTMS $\{1, \overline{1}\}+p$ ESI Full ms [100.00-1000.00]

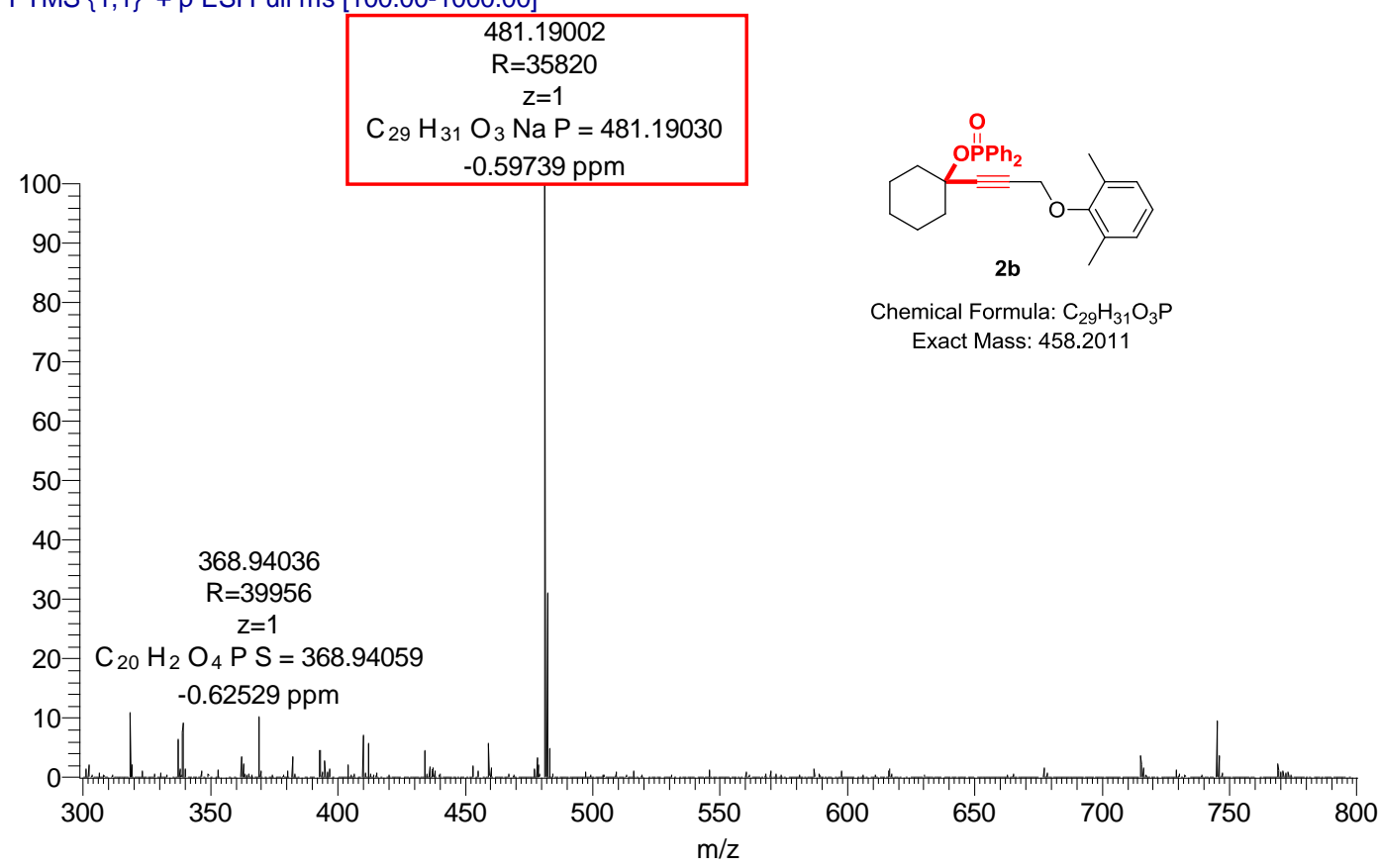

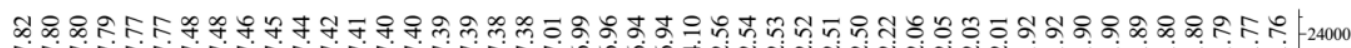

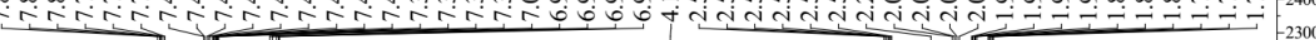

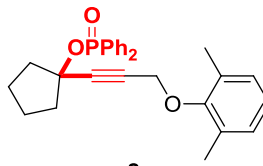

2c

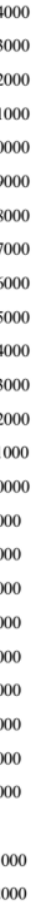



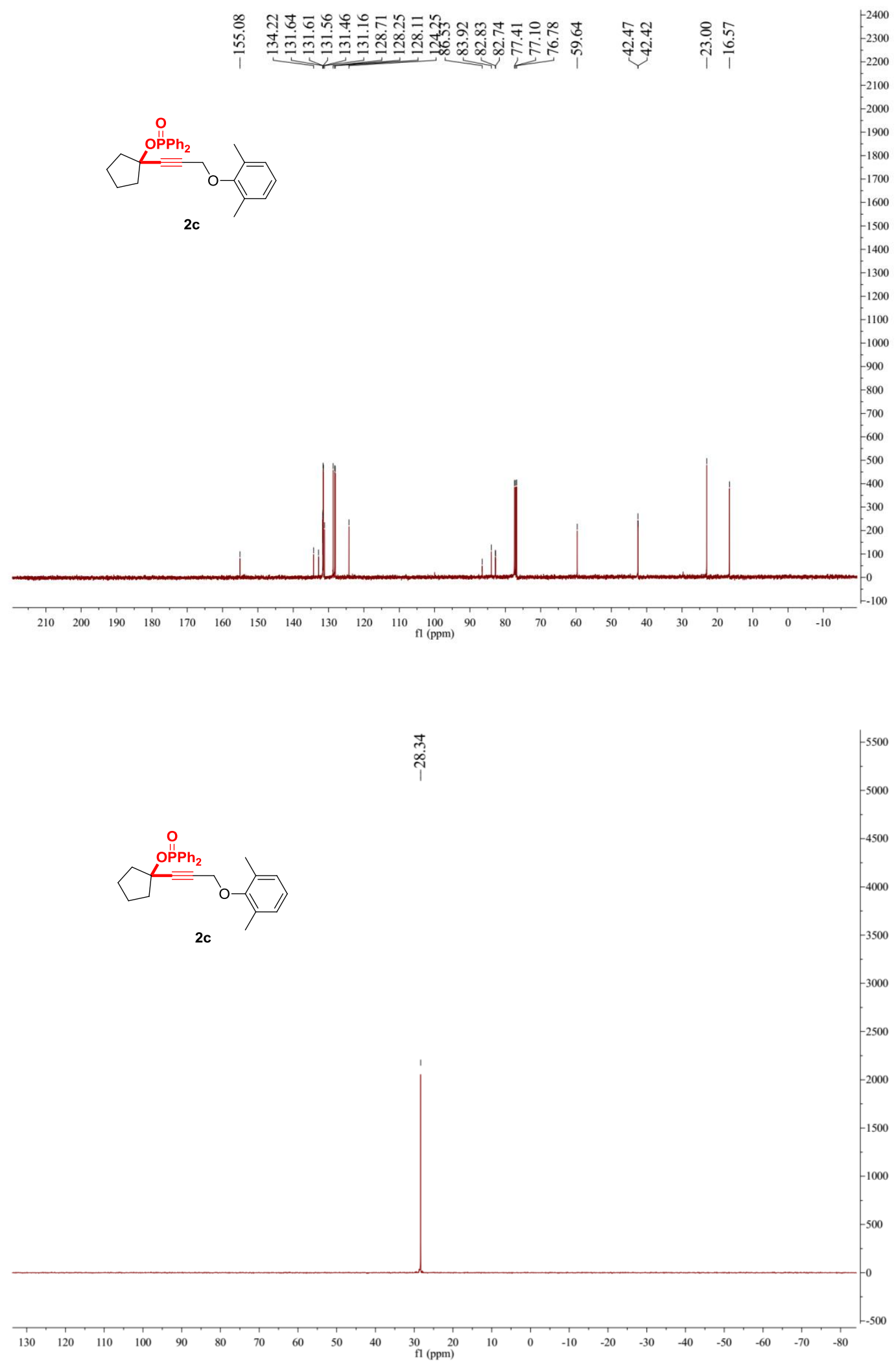
20171124-17 \#44-45 RT: 0.38-0.39 AV: 2 NL: 3.47E6

T: FTMS $\{1,1\}+$ p ESI Full ms [100.00-1000.00]

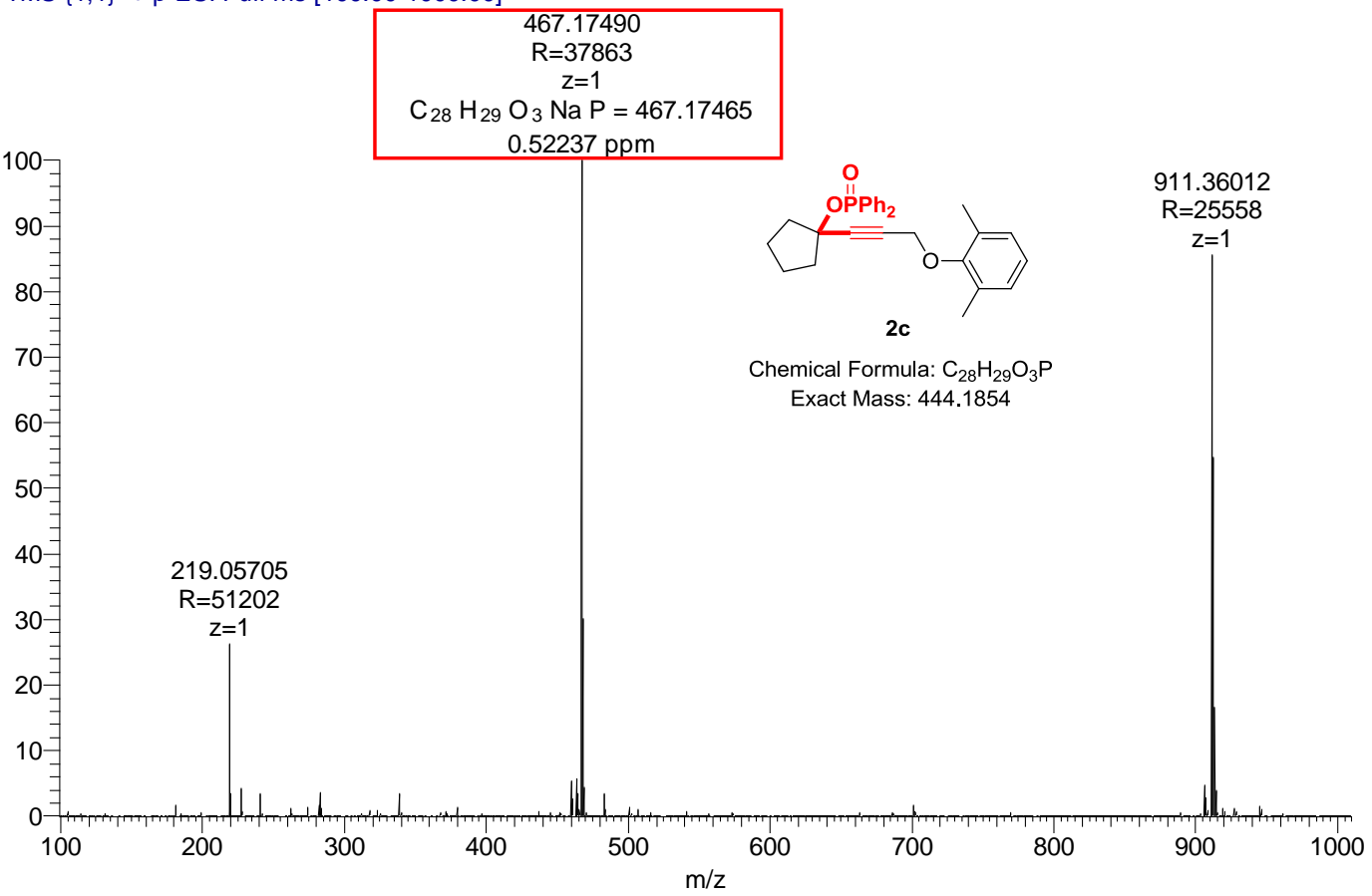

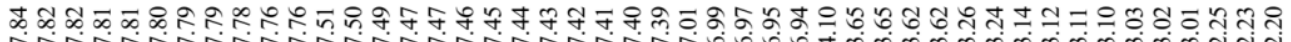

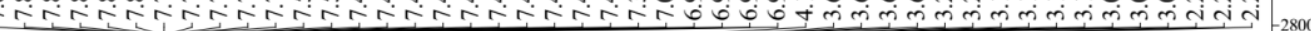<smiles>Cc1cccc(C)c1OCC#CC1(OP(=O)(O)c2ccccc2)CCSC1</smiles>

2d

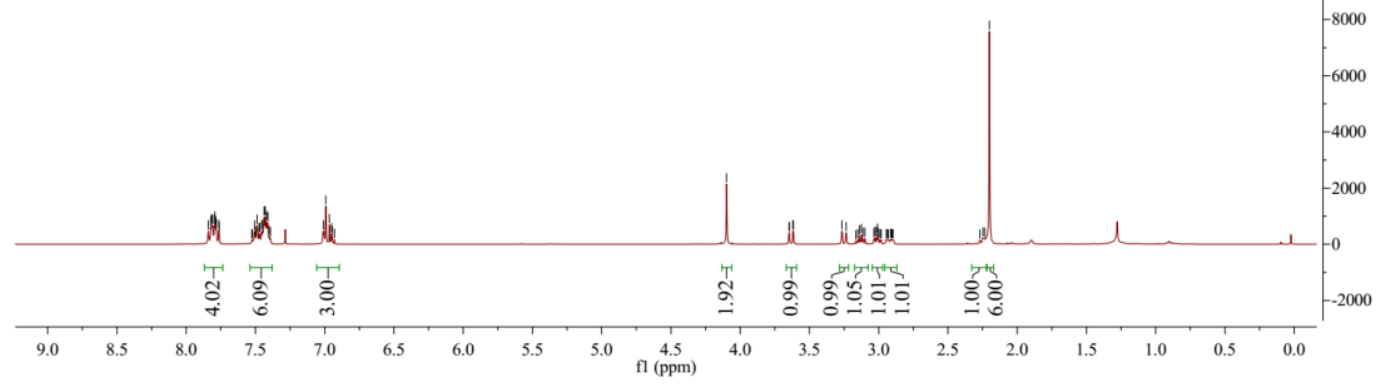



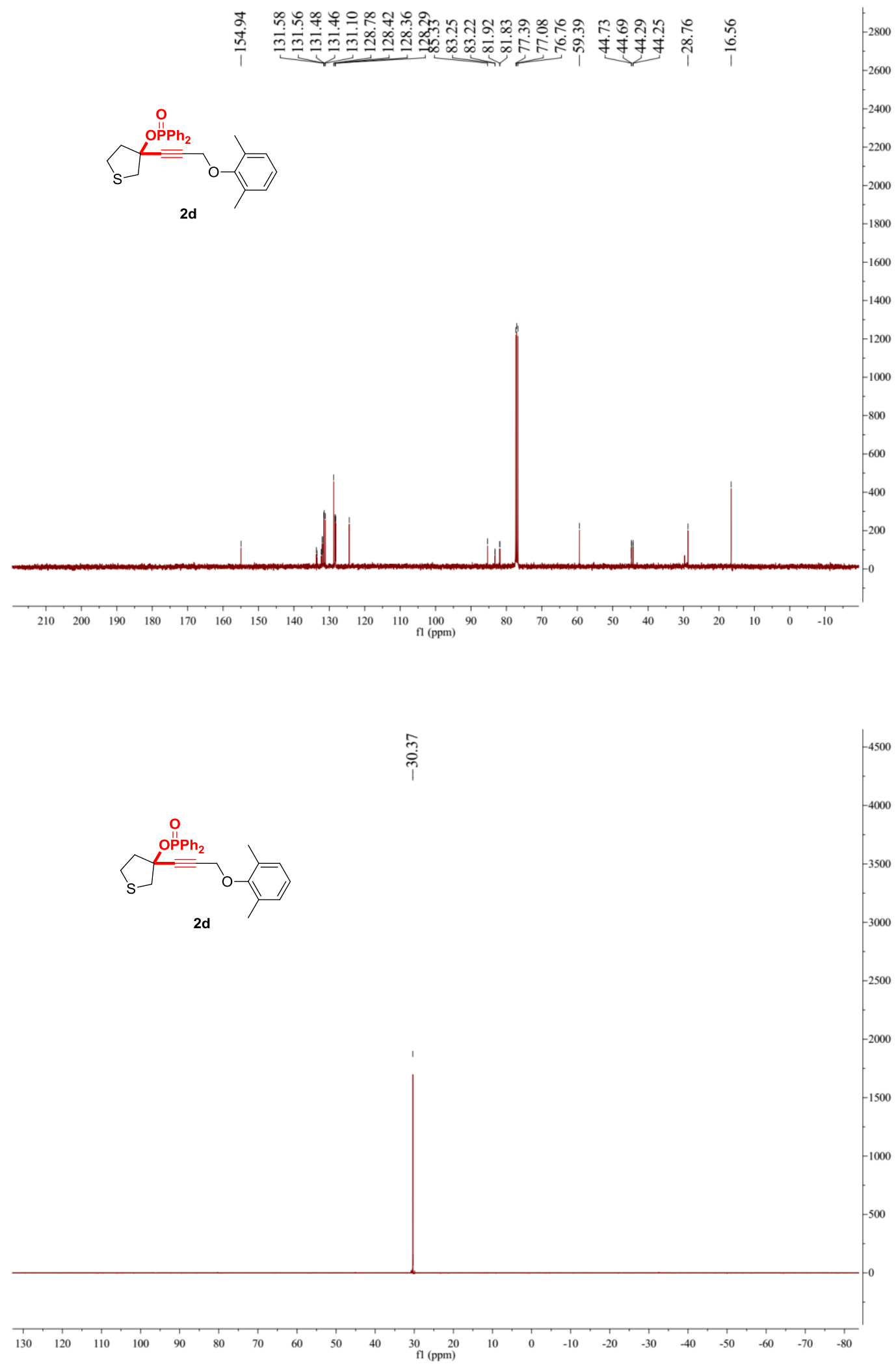
20171124-19 \#32-33 RT: 0.29-0.30 AV: 2 NL: 3.81E6

T: FTMS $\{1,1\}+$ p ESI Full ms [100.00-1000.00]

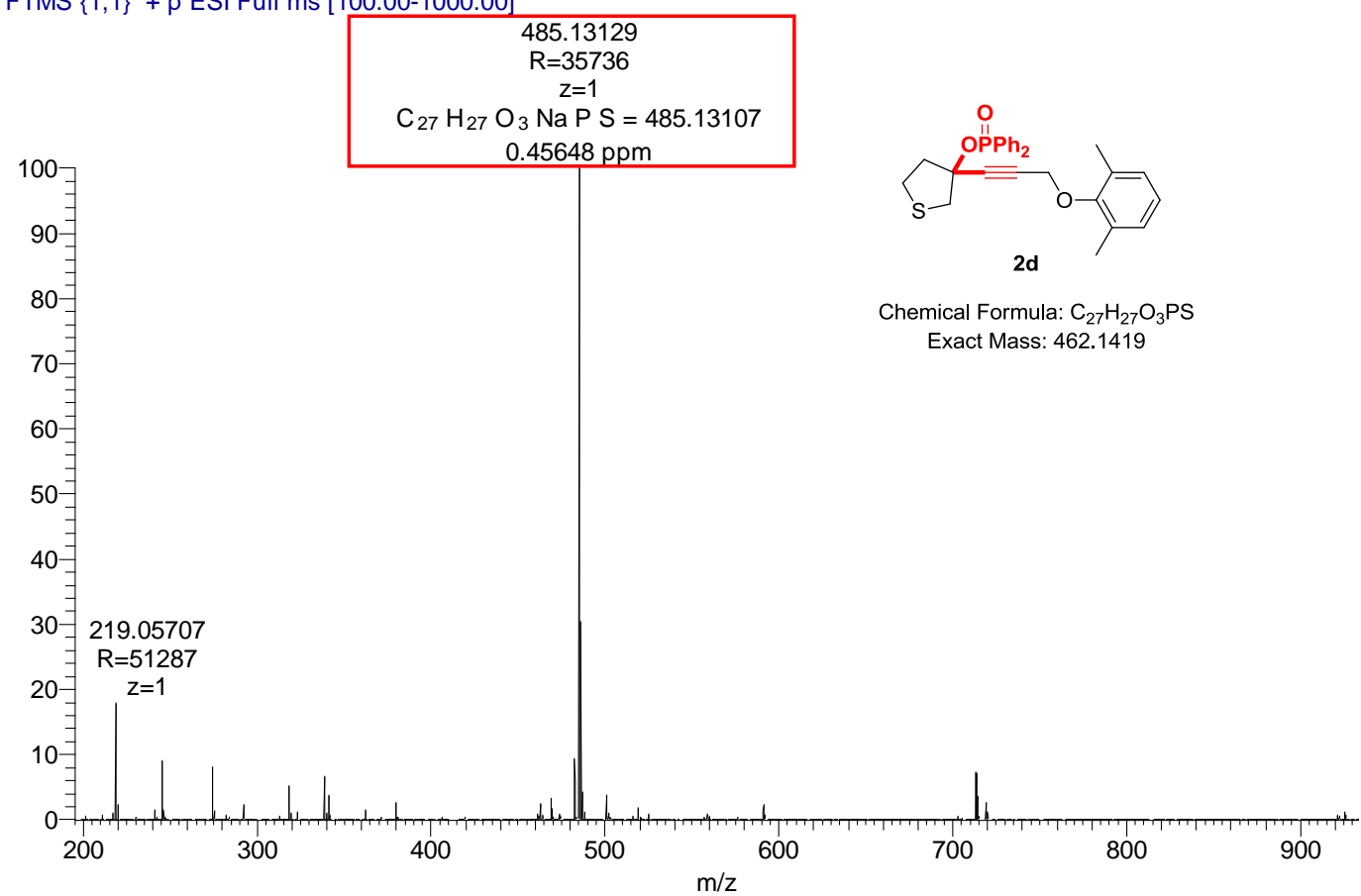

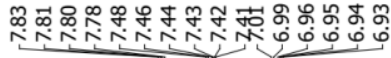
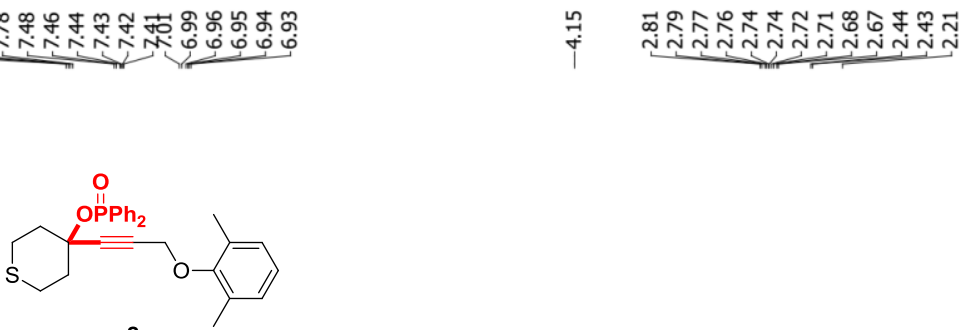

$2 \mathrm{e}$

$-60000$

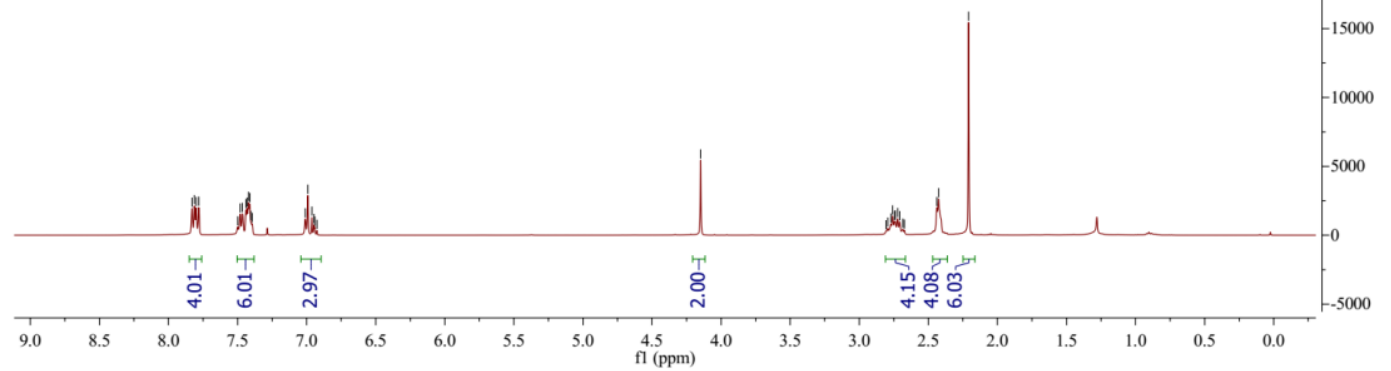




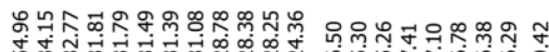

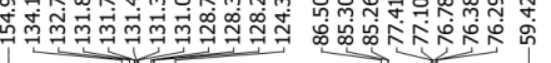
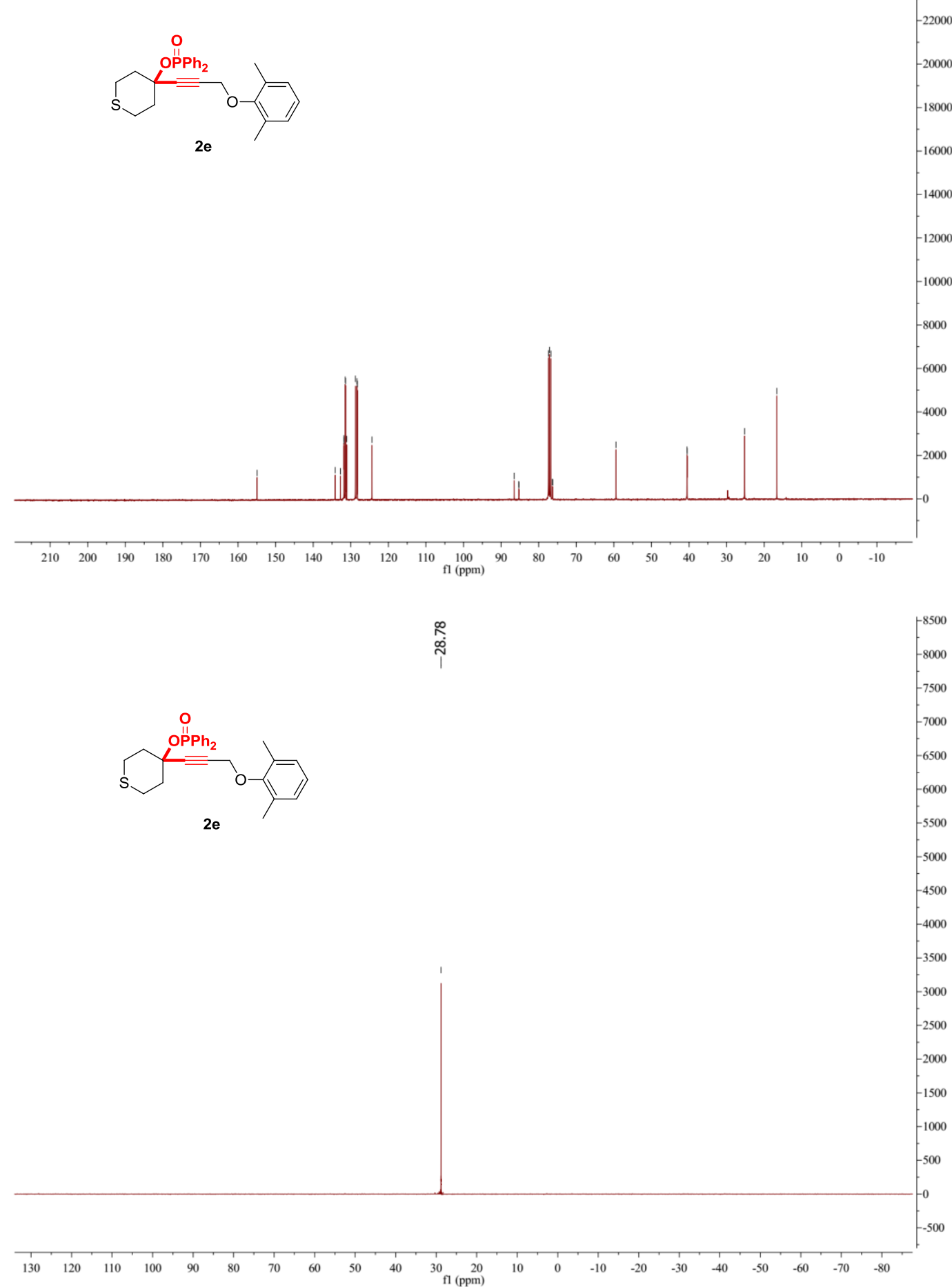
20170618-4 \#41-42 RT: 0.37-0.38 AV: 2 NL: 4.45E5

T: FTMS $\{1,1\}+p$ ESI Full ms [100.00-1000.00]
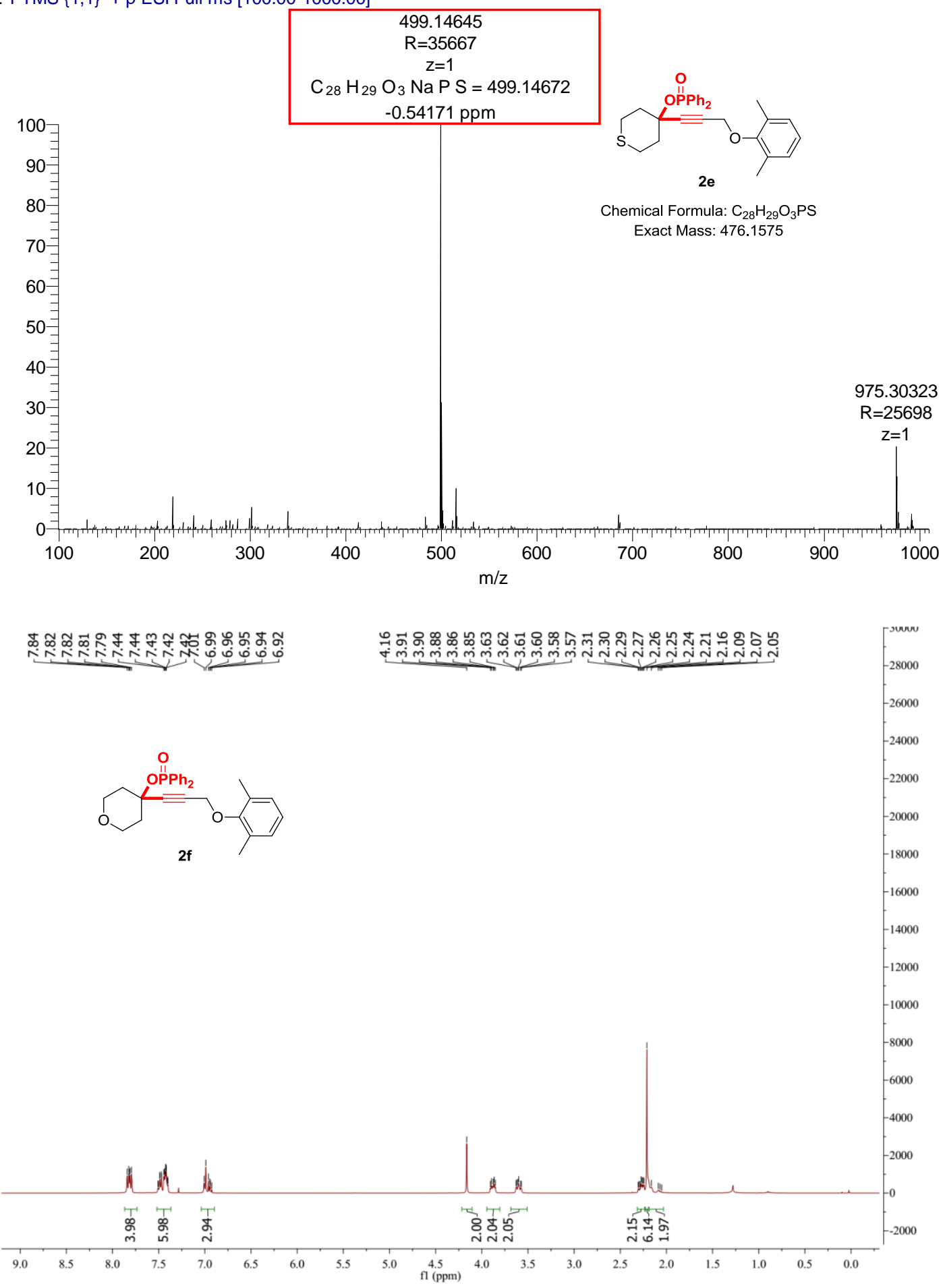

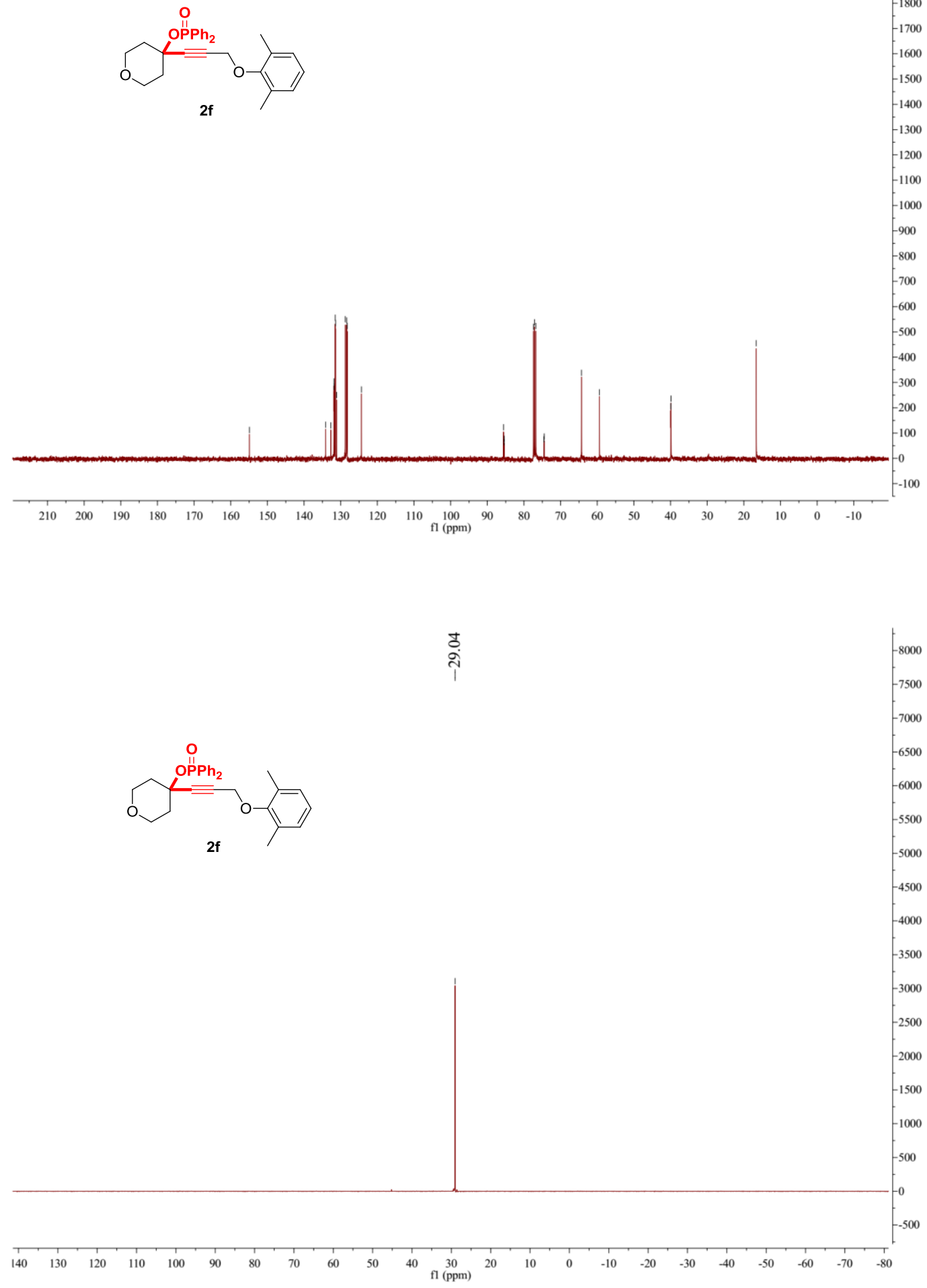
20170618-3 \#51-53 RT: 0.45-0.48 AV: 3 NL: 8.17E5

T: FTMS $\{1,1\}+p$ ESI Full ms [100.00-1000.00]
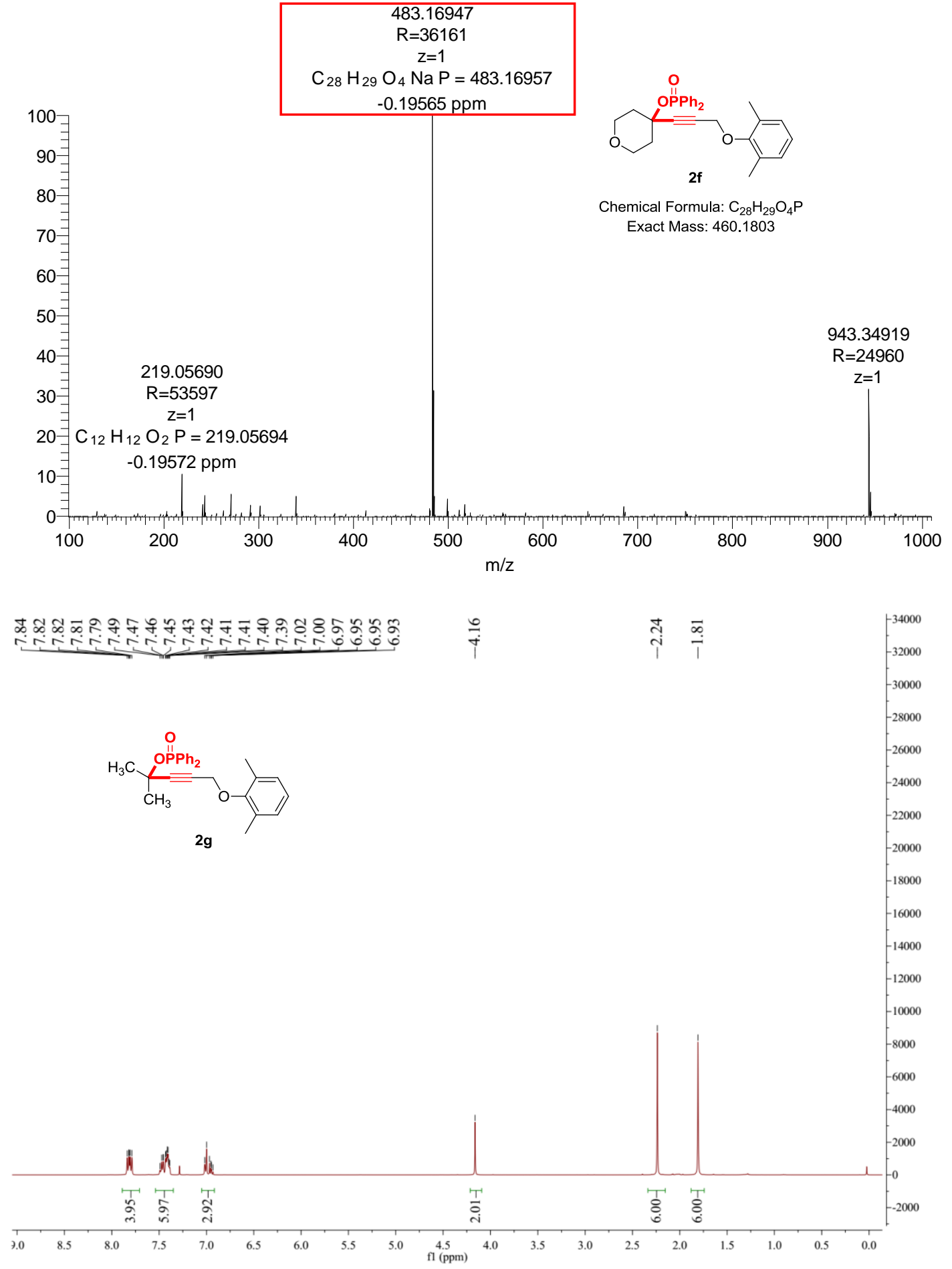

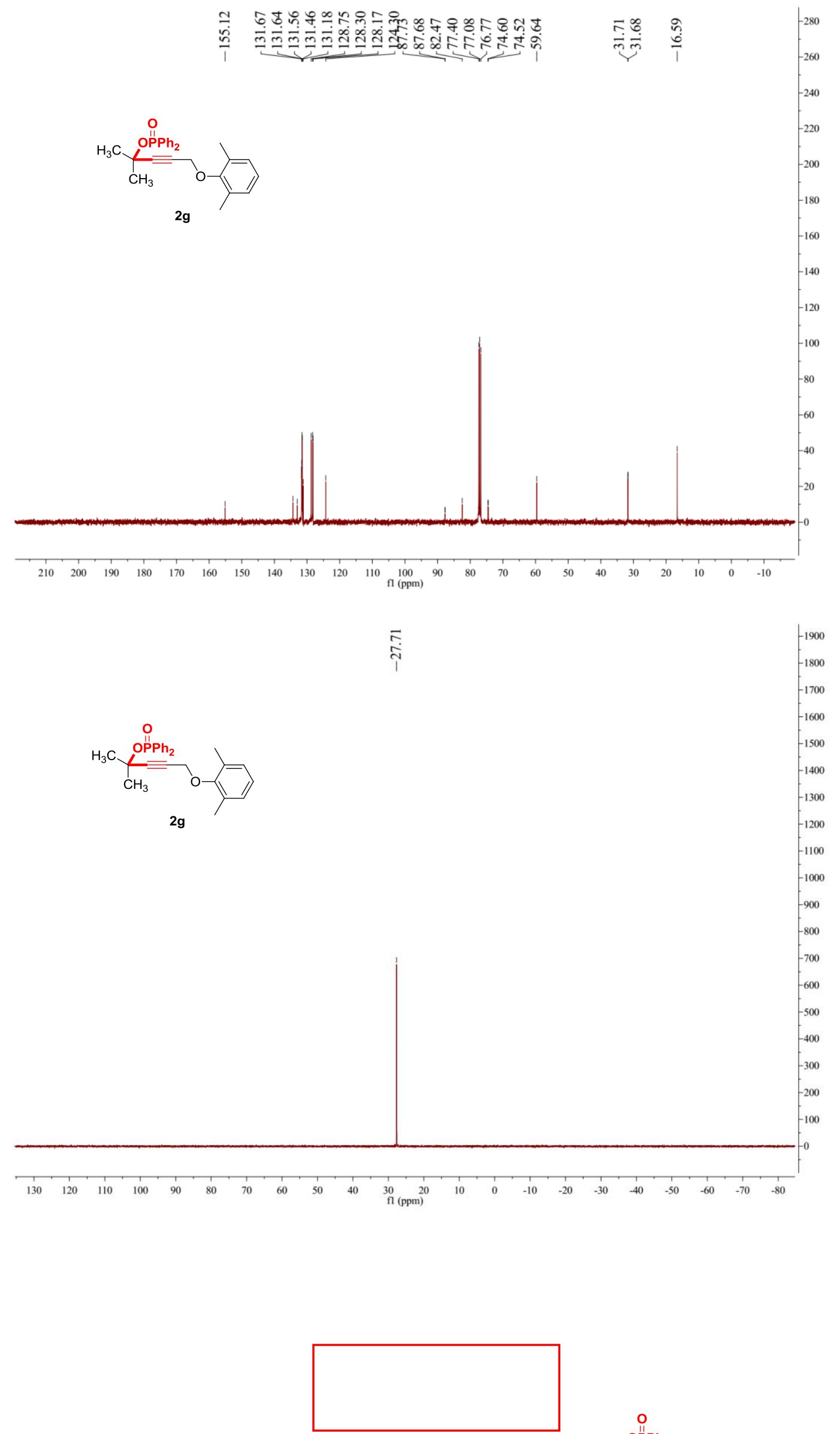

$90 / 143$

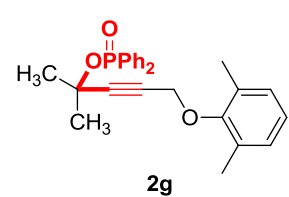

Chemical Formula: $\mathrm{C}_{26} \mathrm{H}_{27} \mathrm{O}_{3} \mathrm{P}$ Exact Mass: 418.1698 
20170505-44_170506153344 \#13-15 RT: 0.13-0.15 AV: 3 NL: 8.32E5

T: FTMS $\{1, \overline{1}\}+p$ ESI Full ms [100.00-1000.00]

219.05685

$\mathrm{C}_{12} \mathrm{H}_{12} \mathrm{O}_{2} \mathrm{P}=219.05694$

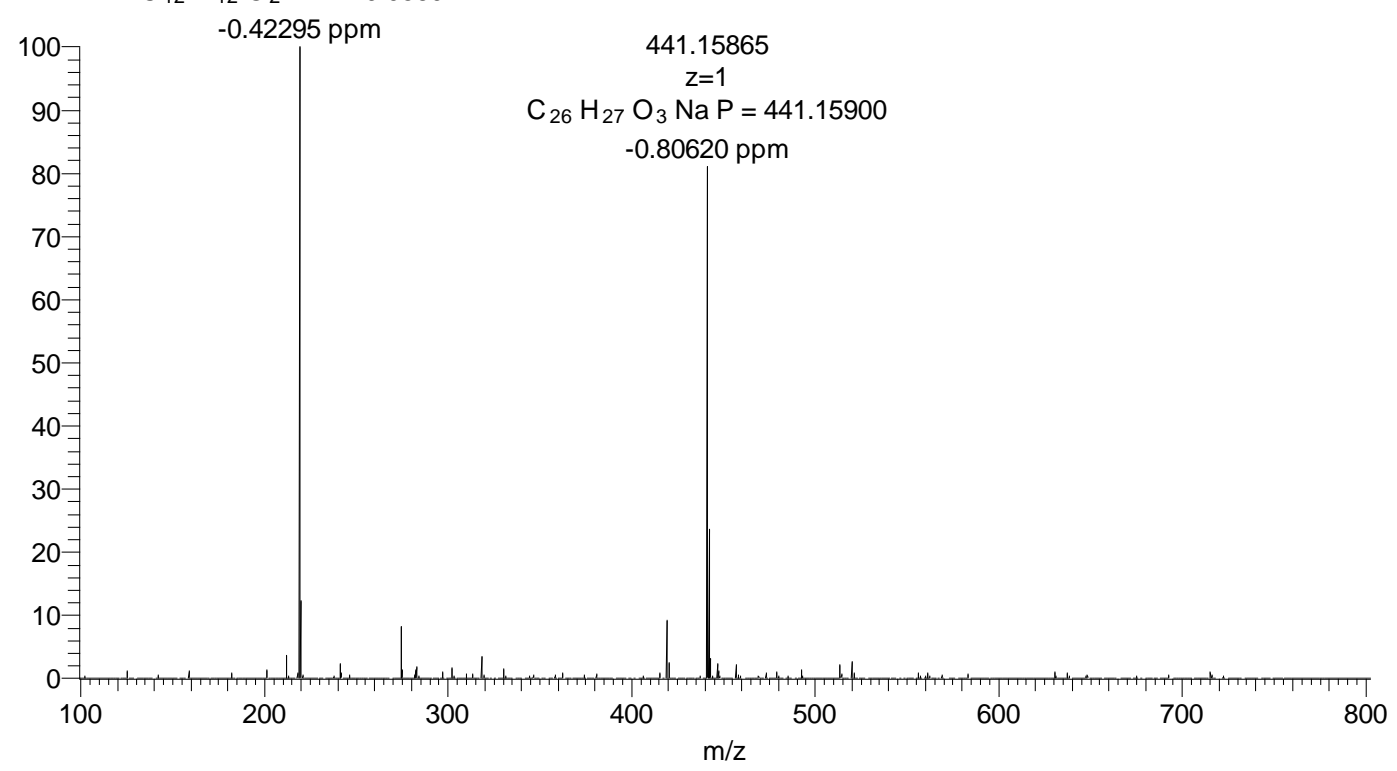

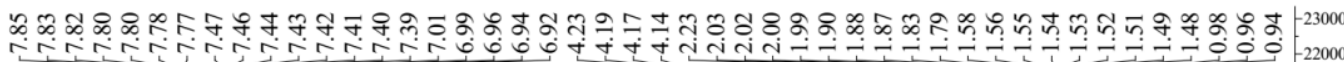

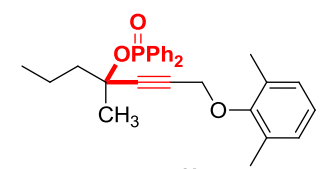

$2 h$

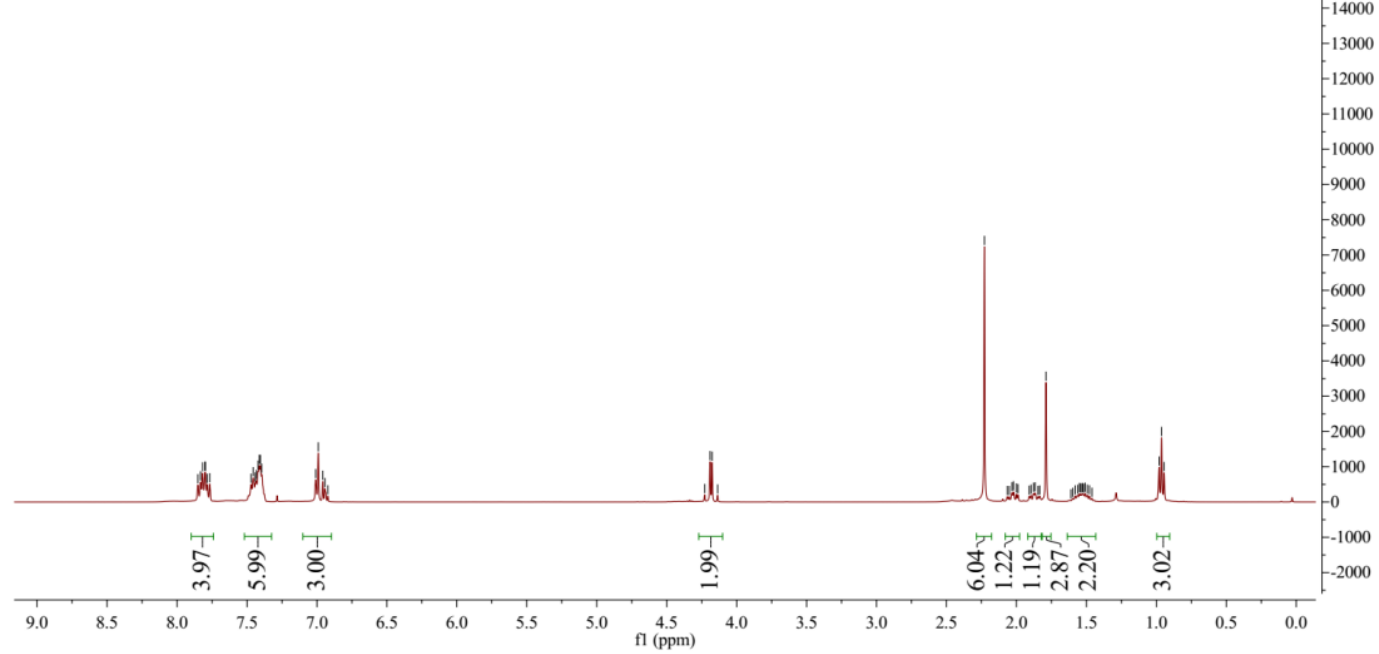




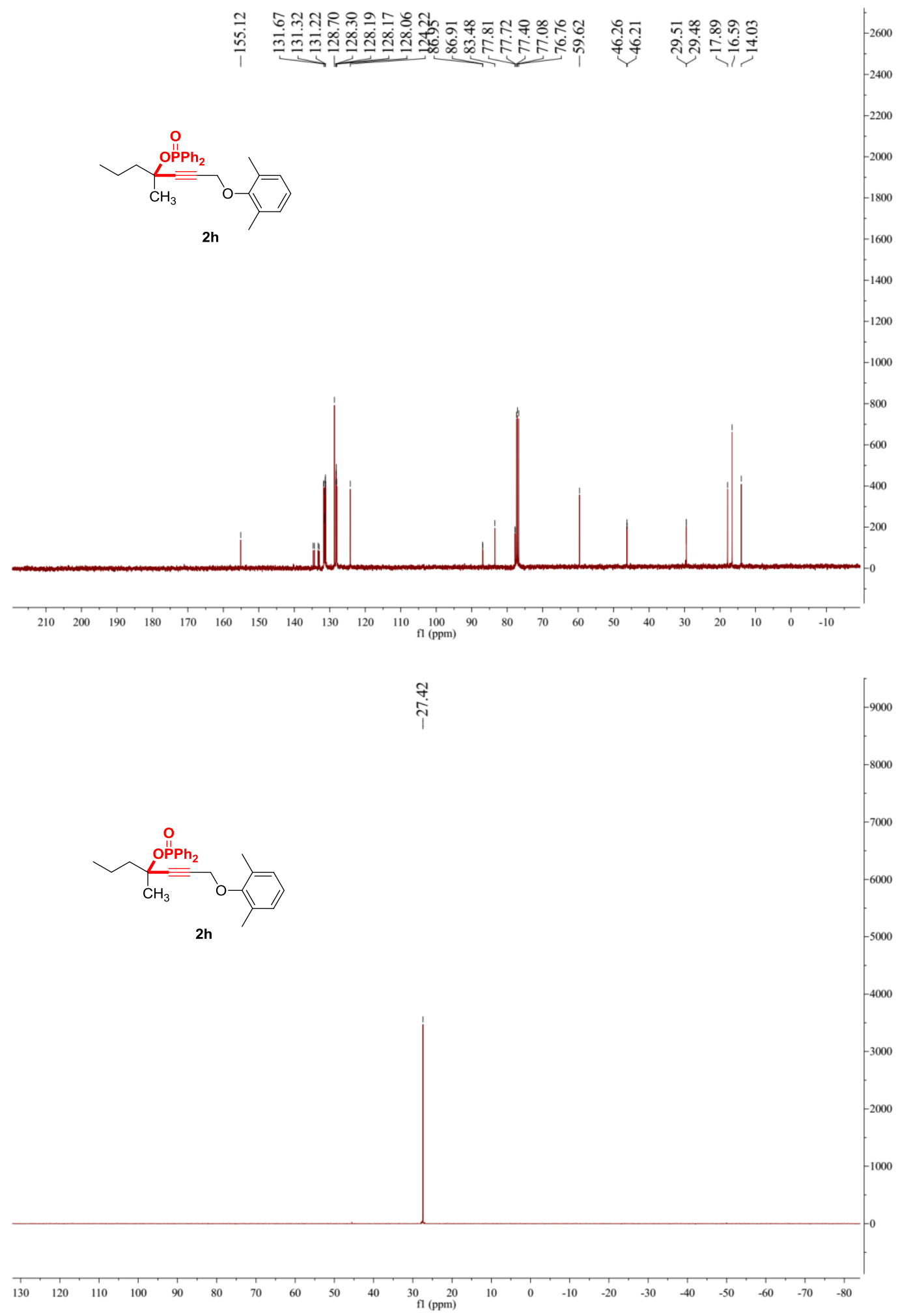


20170505-45_170506154924 \#46-47 RT: 0.44-0.45 AV: 2 NL: 3.82E5 T: FTMS $\{1, \overline{1}\}+p$ ESI Full ms [100.00-1000.00]

219.05692
$R=53595$

$\mathrm{Z}=1$

$\mathrm{C}_{12} \mathrm{H}_{12} \mathrm{O}_{2} \mathrm{P}=219.05694$
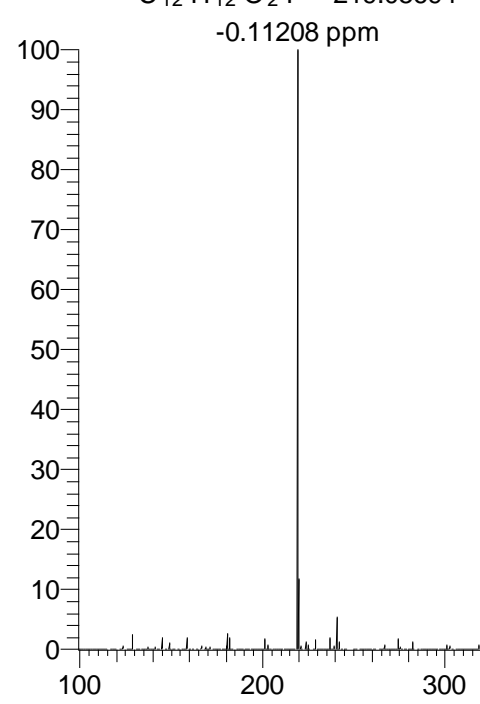

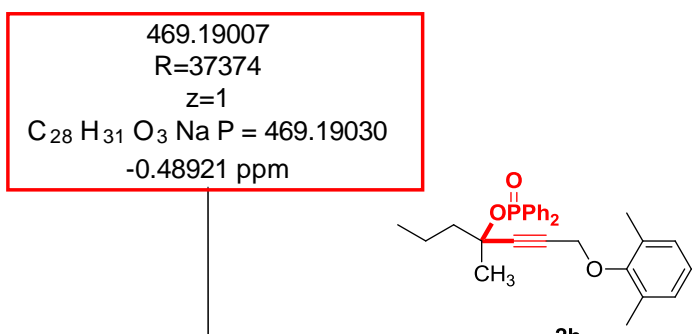

Chemical Formula: $\mathrm{C}_{28} \mathrm{H}_{31} \mathrm{O}_{3} \mathrm{P}$ Exact Mass: 446.2011

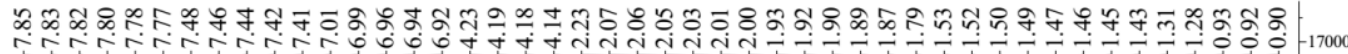

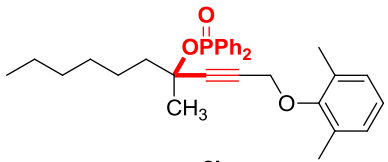

2i

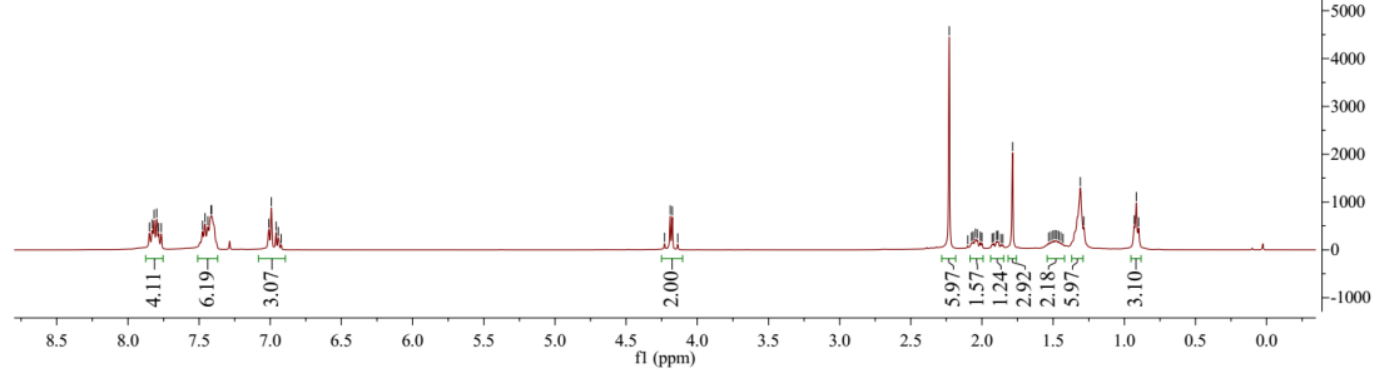



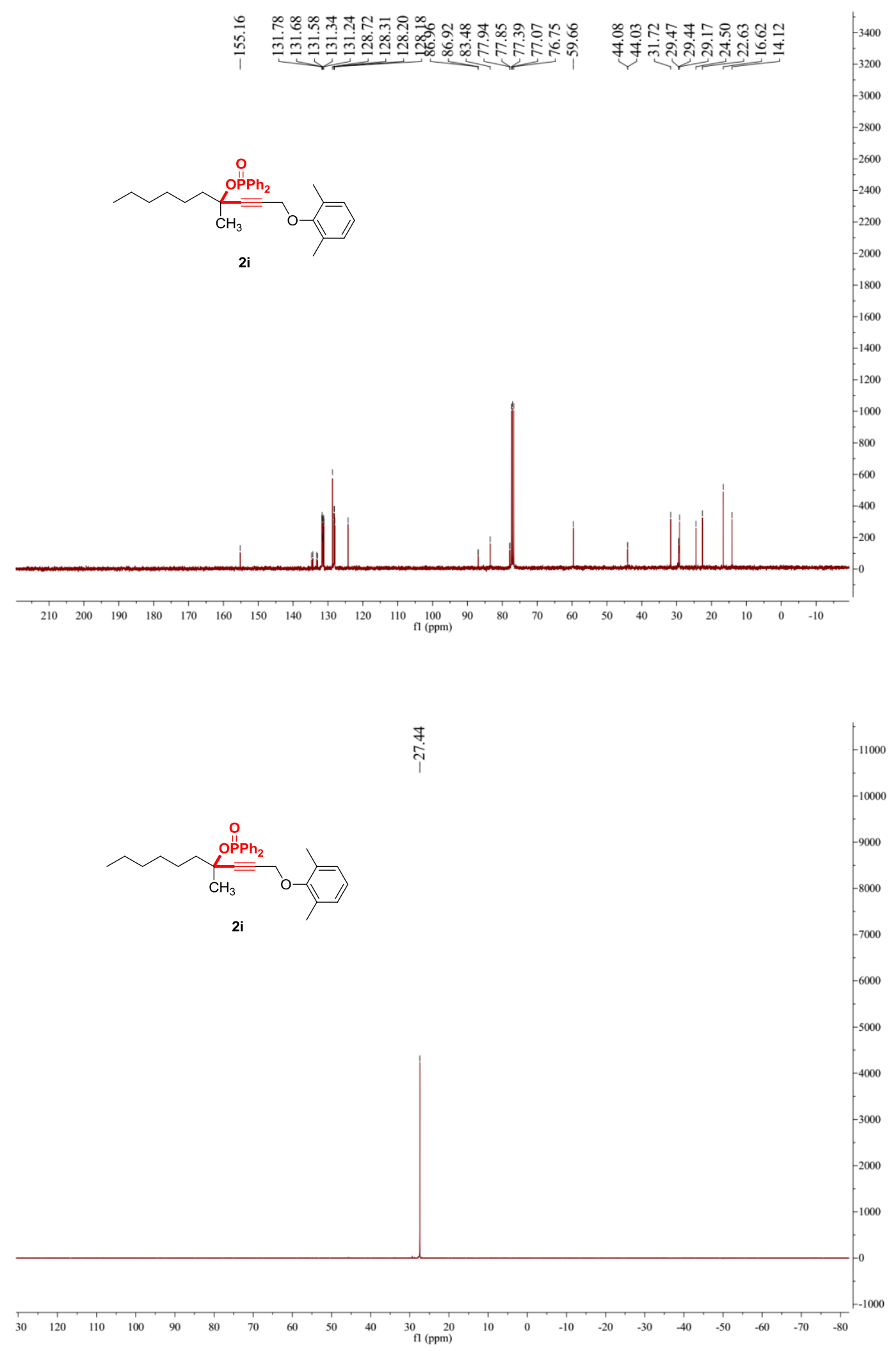
20170618-2 \#64-69 RT: 0.56-0.62 AV: 6 NL: 6.56E5

T: FTMS $\{1,1\}+p$ ESI Full ms [100.00-1000.00]

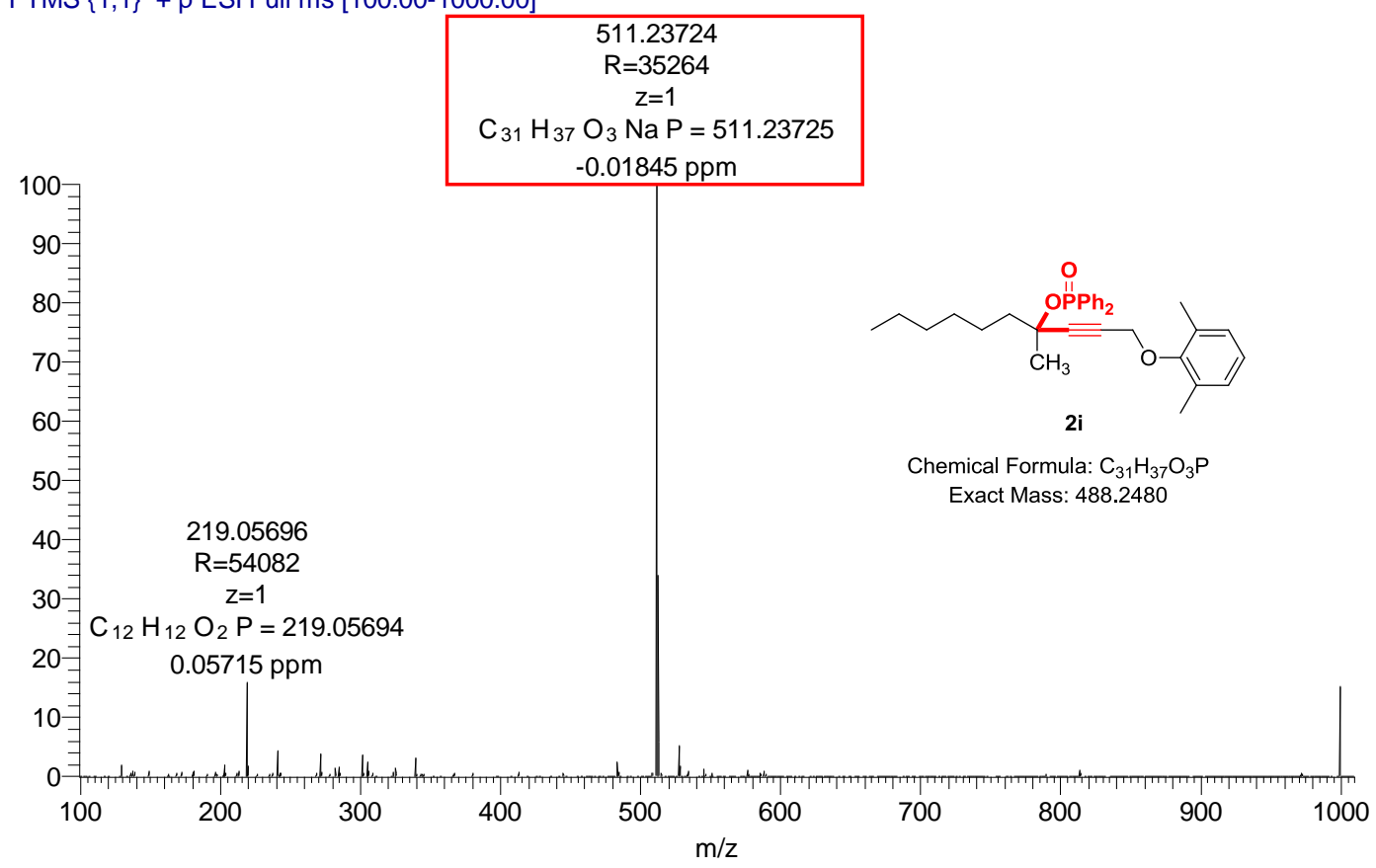

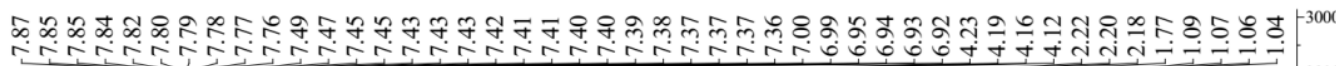

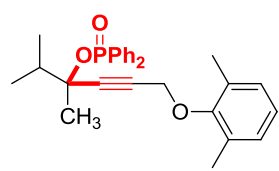

2j

\section{0}

26000

22000

20000

8000

16000

$-14000$

$-12000$

$-10000$

$-8000$

6000

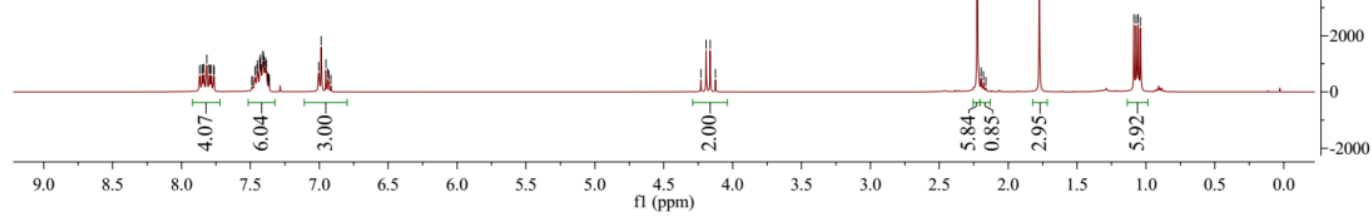



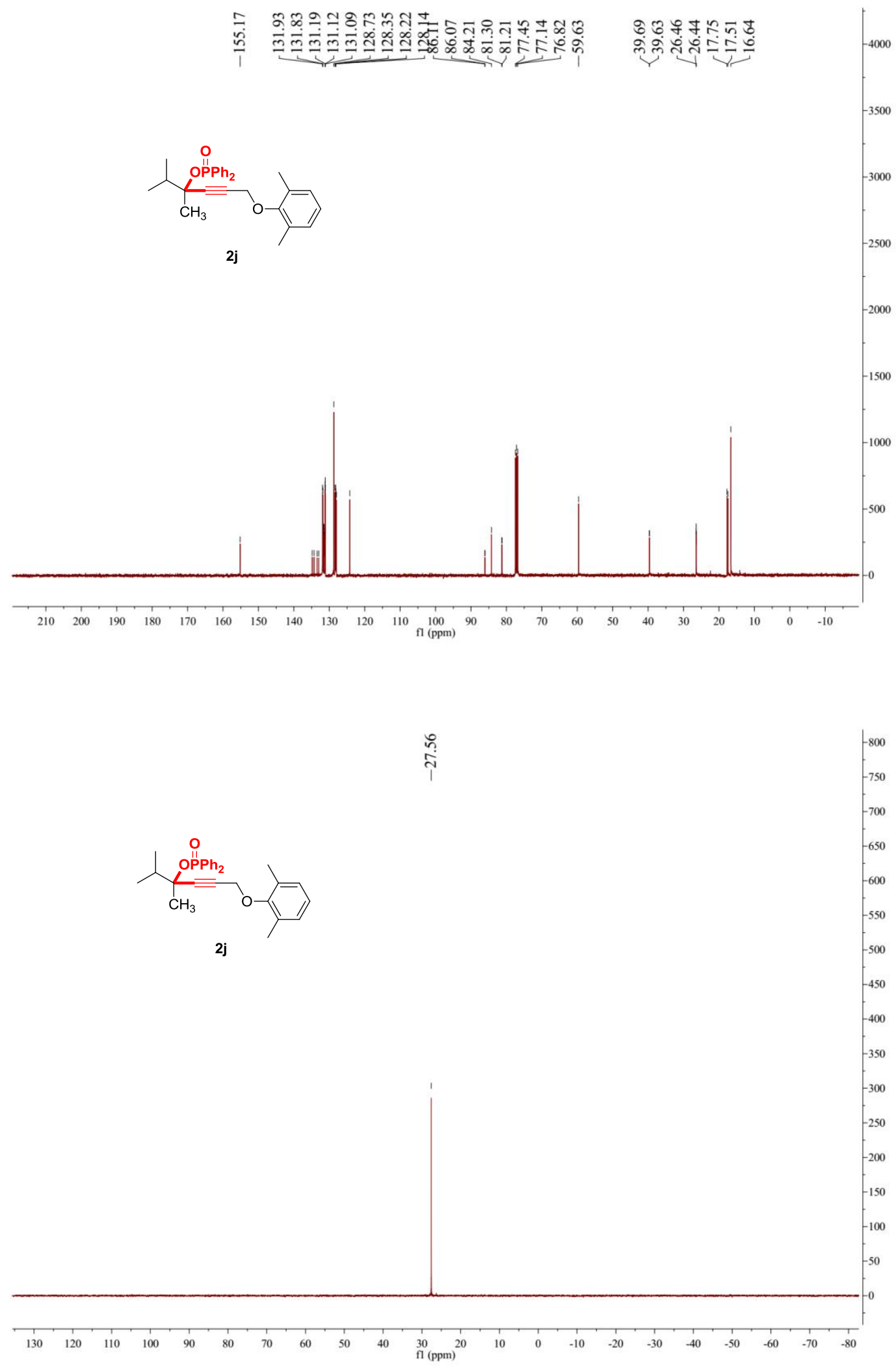
20170505-47_170506162034 \#37-39 RT: 0.30-0.32 AV: 3 NL: 1.79E6

T: FTMS $\{1, \overline{1}\}+p$ ESI Full ms [100.00-1000.00]

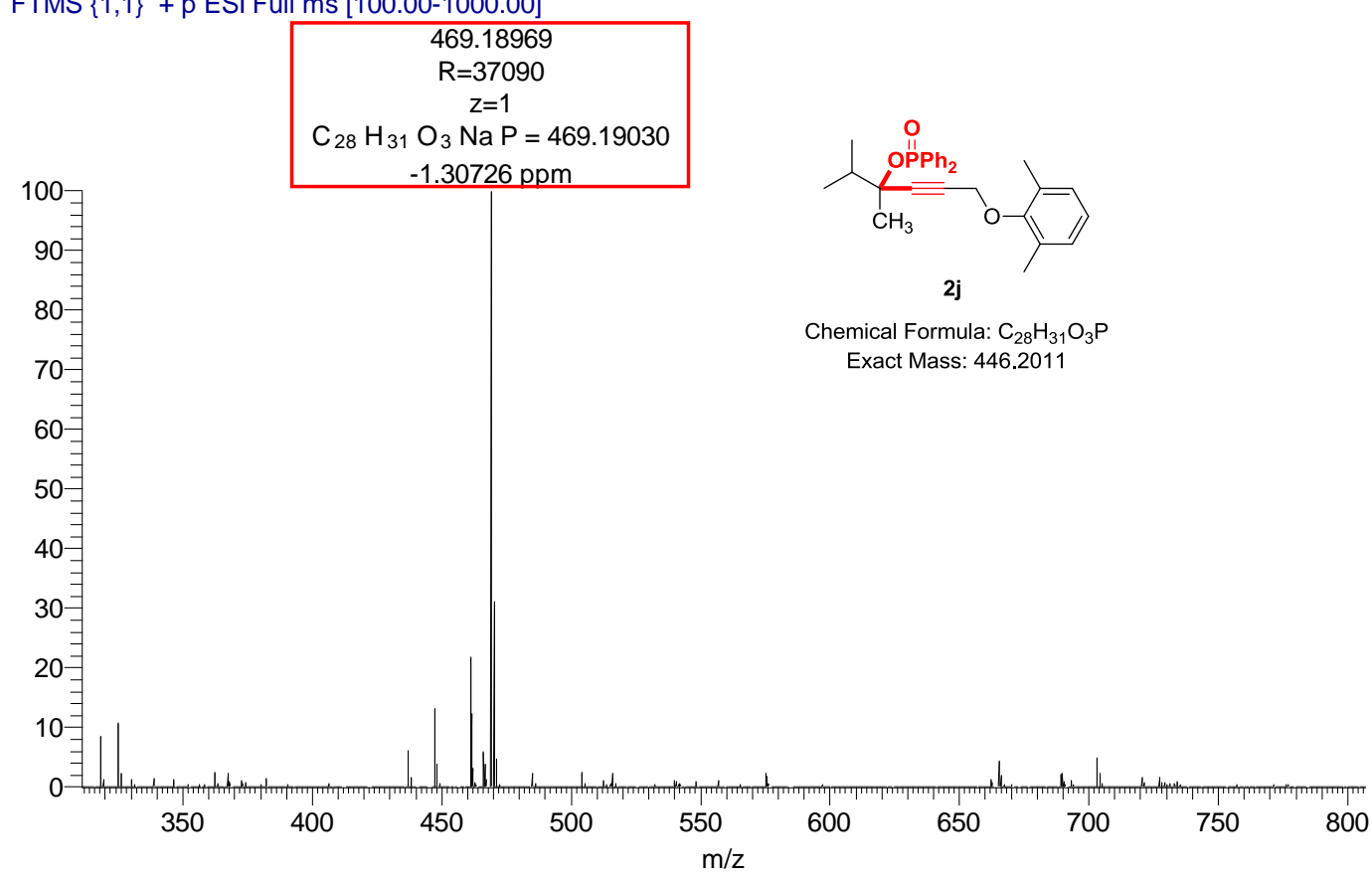

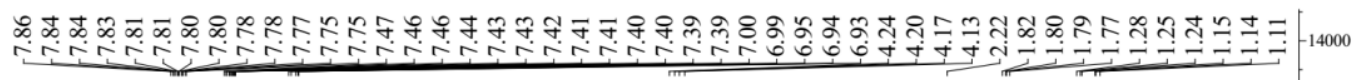

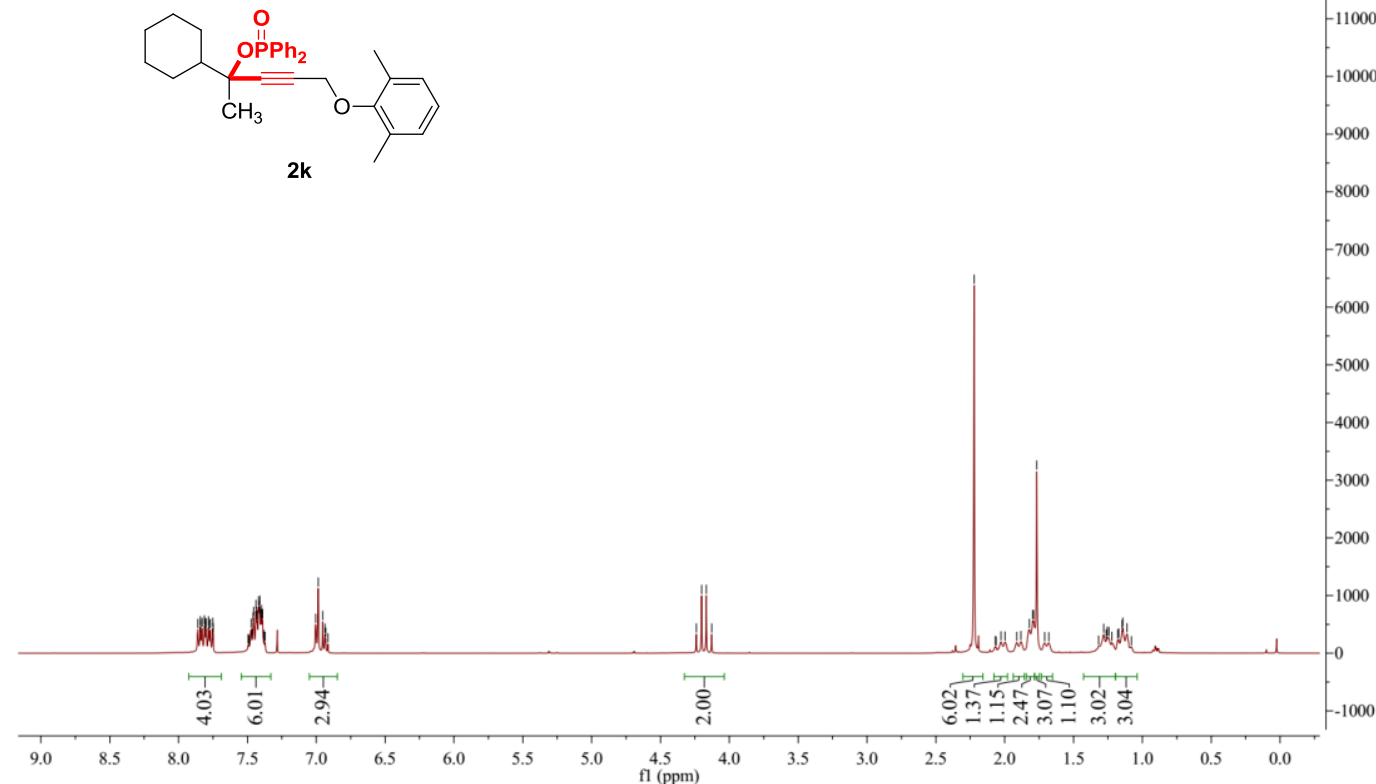



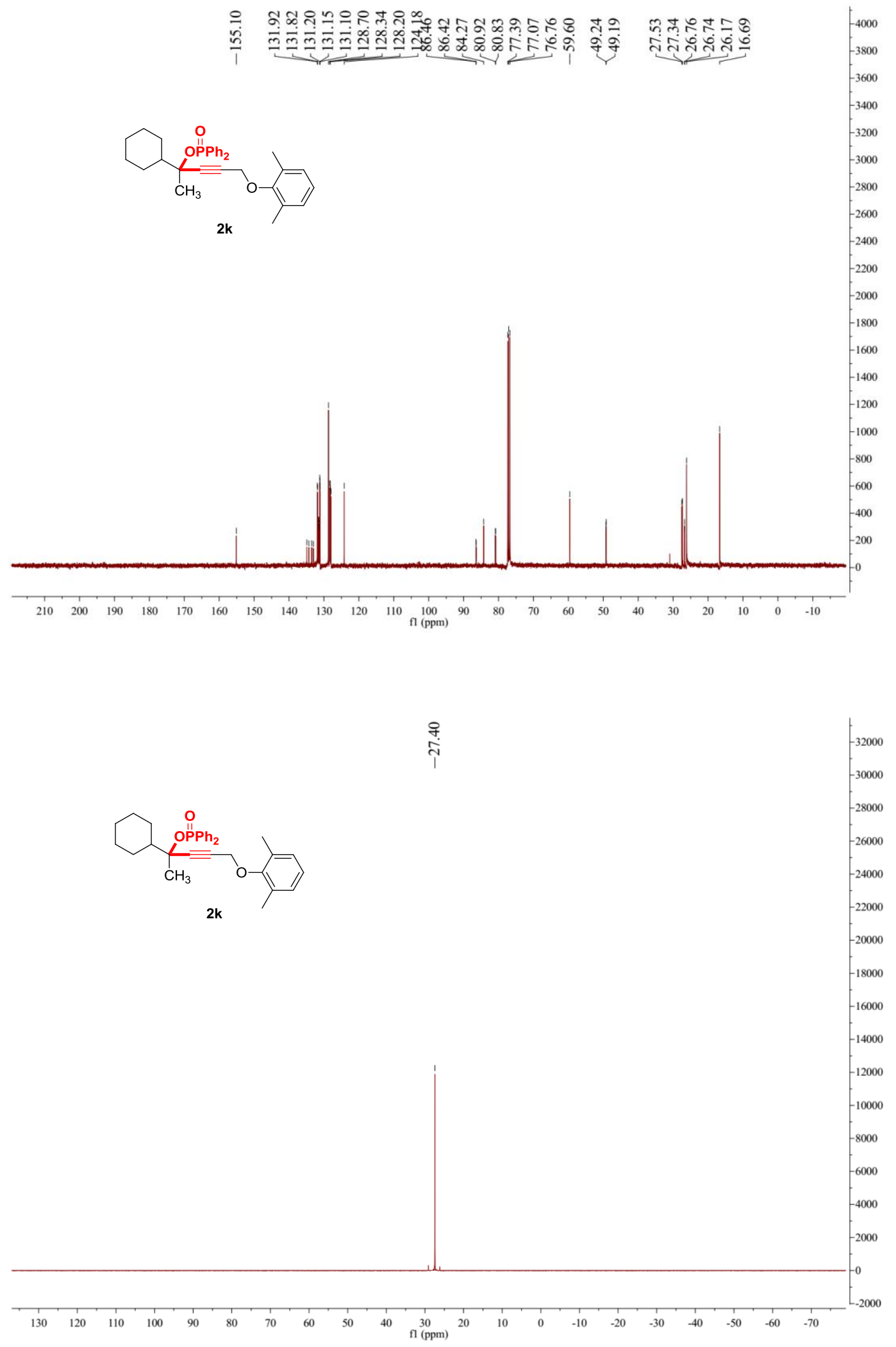
20170505-48_170506163607 \#53-58 RT: 0.42-0.47 AV: 6 NL: 4.65E5

T: FTMS $\{1, \overline{1}\}+p$ ESI Full ms [100.00-1000.00]

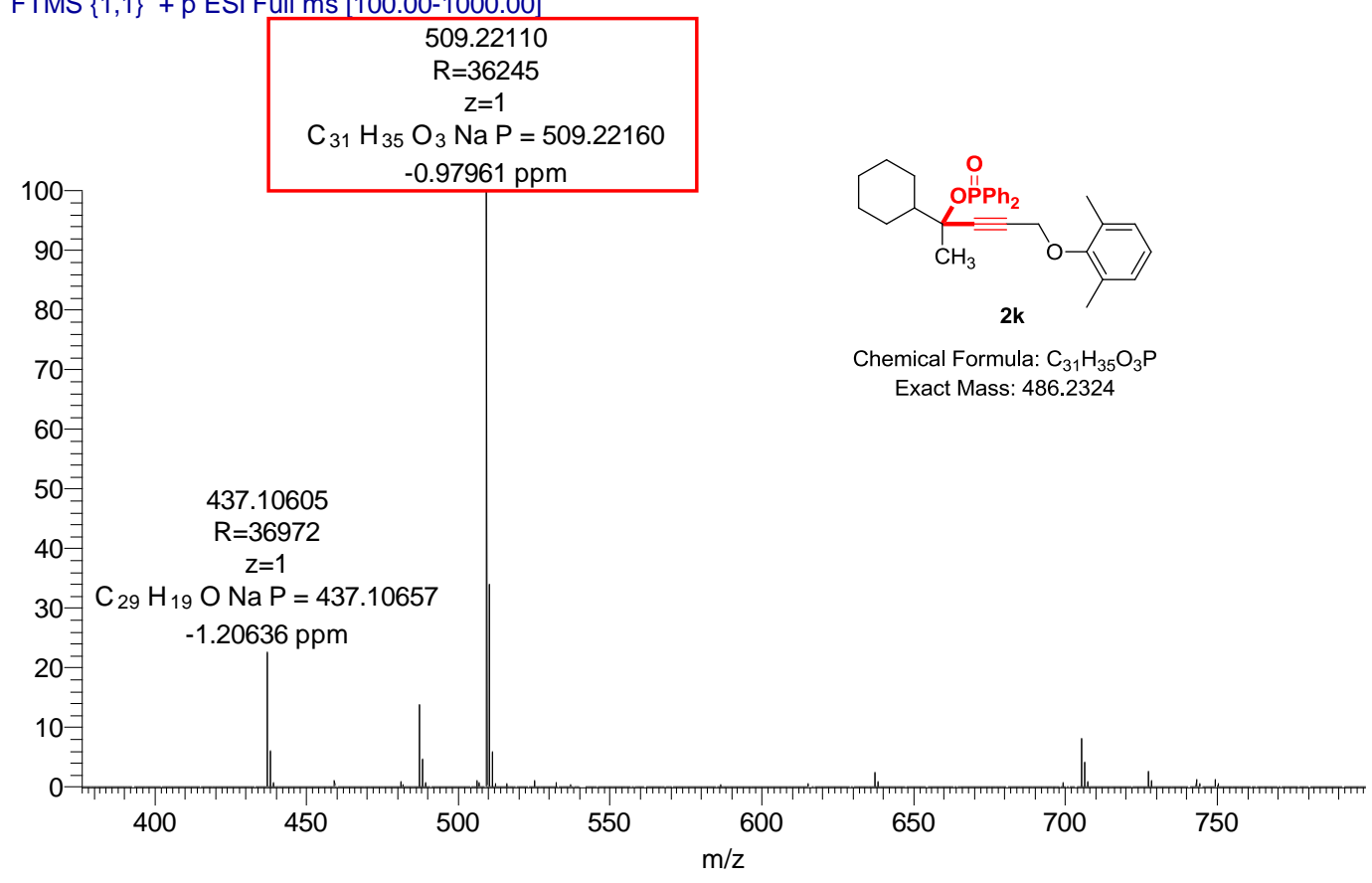

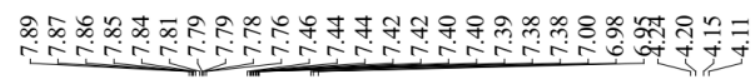

กิ

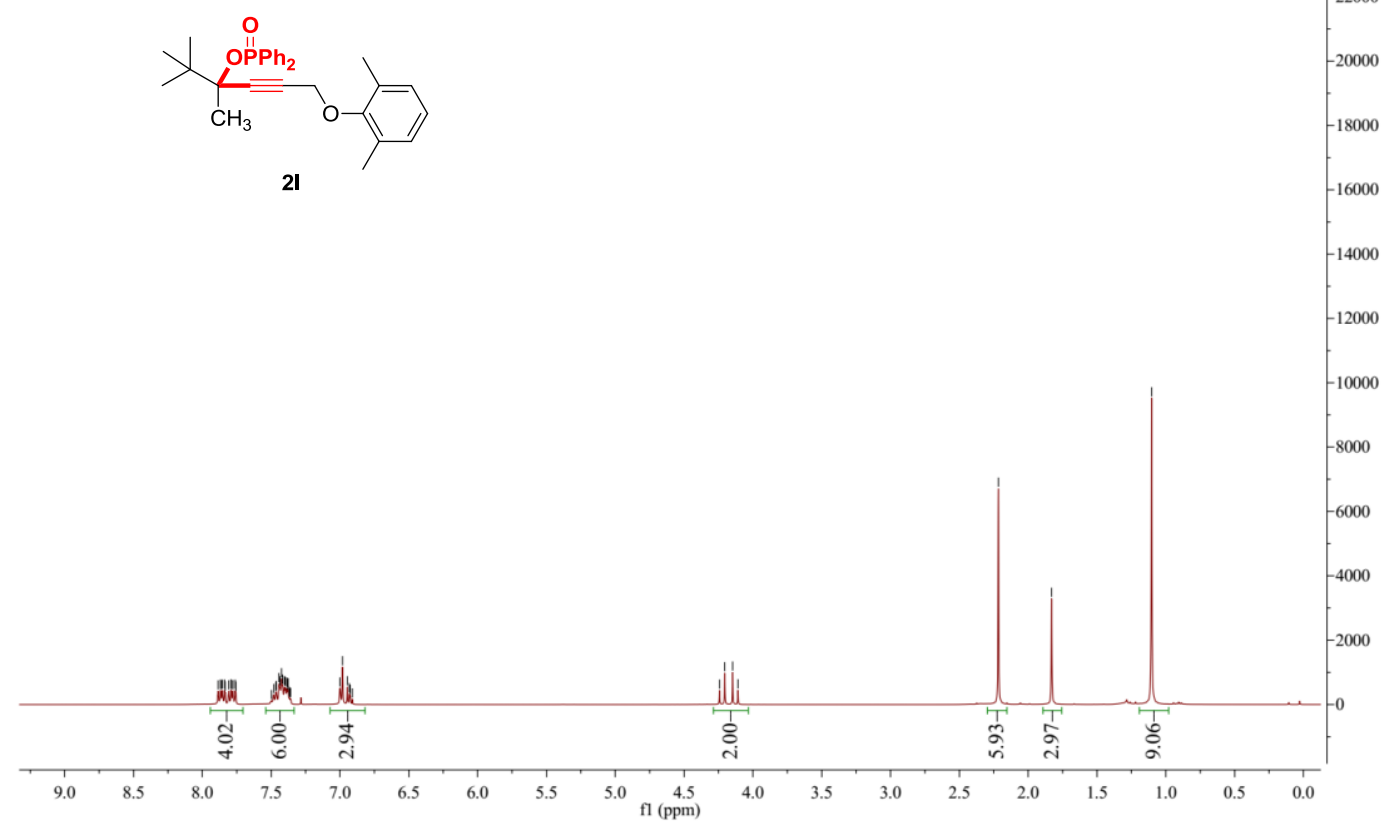

2

$R=36245$

$-0.97961 \mathrm{ppm}$

emical Formula: $\mathrm{C}_{31} \mathrm{H}_{35} \mathrm{O}_{3} \mathrm{P}$

Exact Mass: 486.2324 





20170618-1 \#54-56 RT: 0.44-0.46 AV: 3 NL: 1.32E6

$\mathrm{T}: \mathrm{FTMS}\{1,1\}+\mathrm{p}$ ESI Full ms $[100.00-1000.00]$
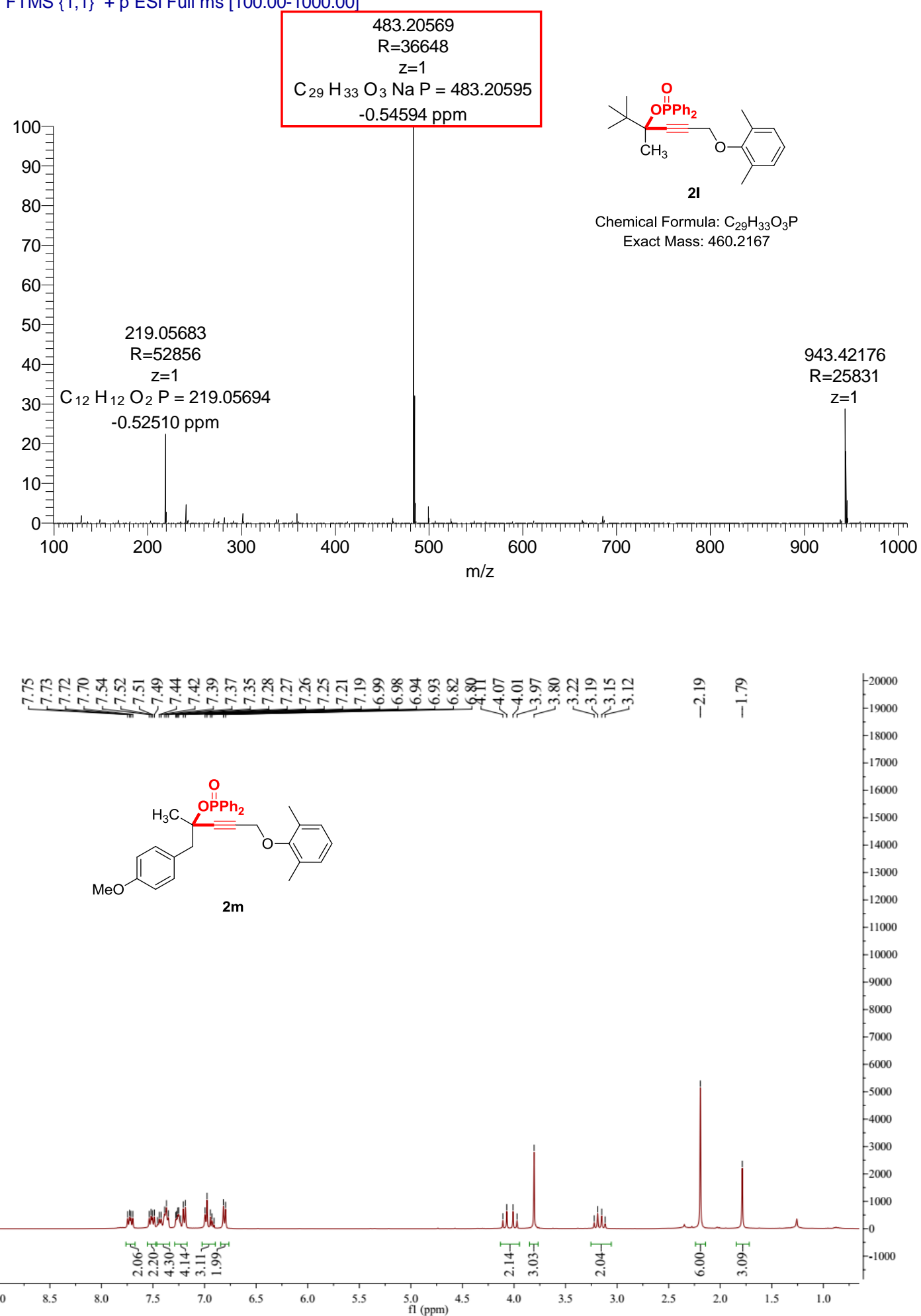


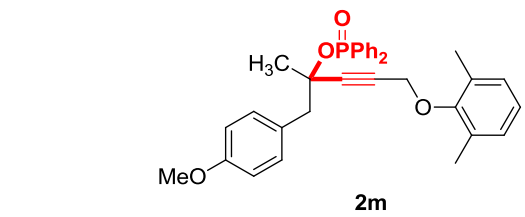

$2 m$
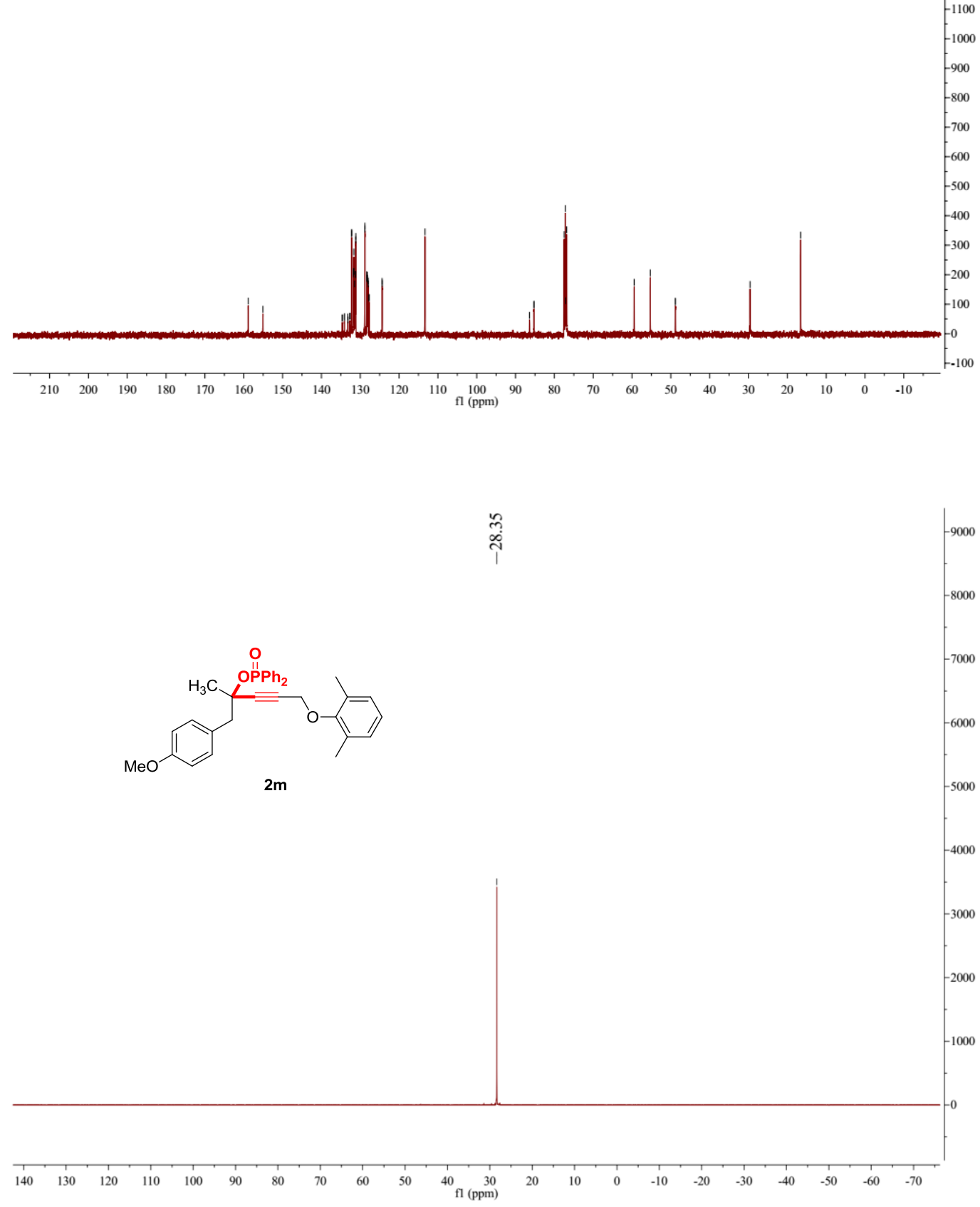
20170618-9 \#50-51 RT: 0.44-0.45 AV: 2 NL: 1.12E6

T: FTMS $\{1,1\}+p$ ESI Full ms $[100.00-1000.00]$
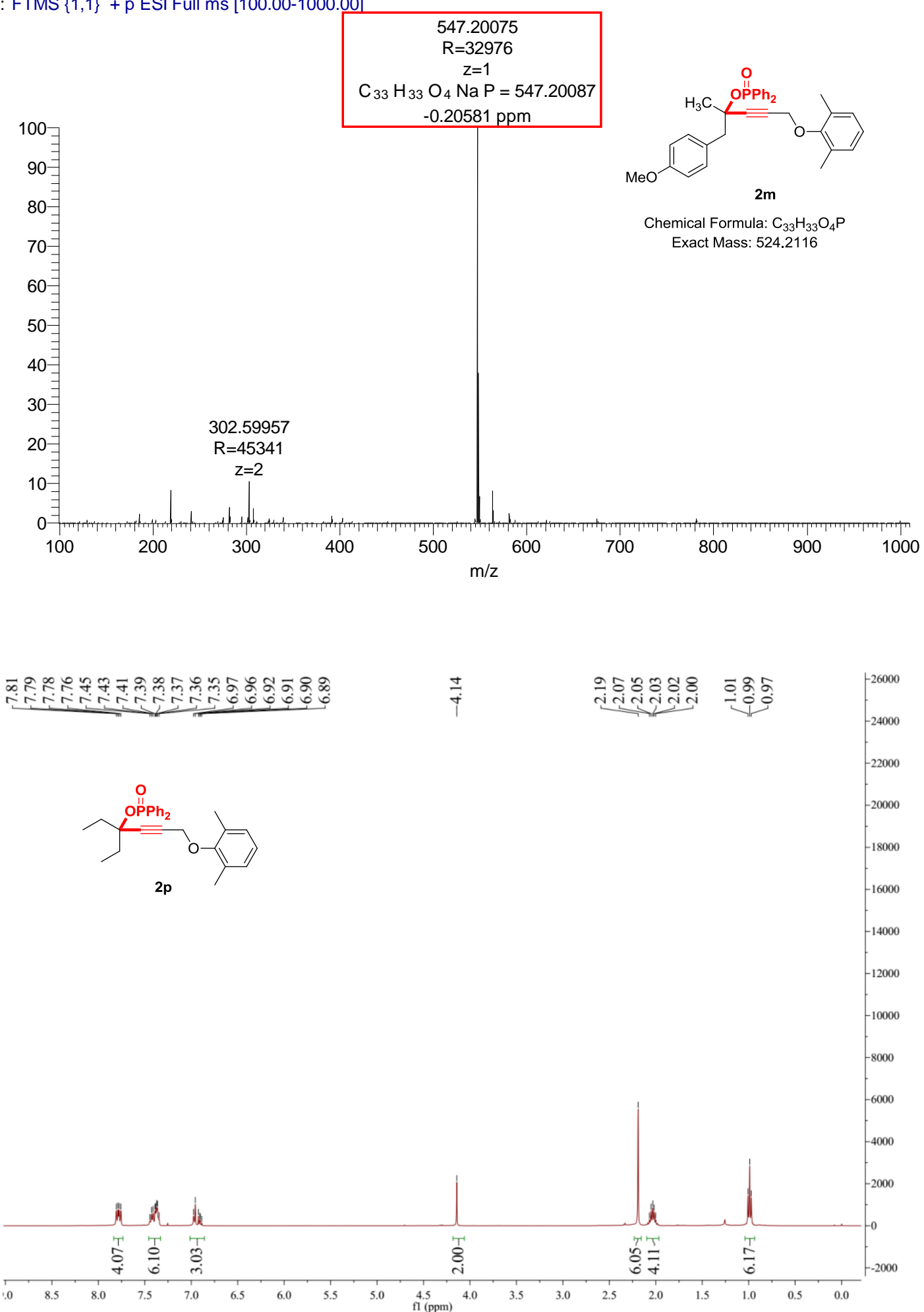


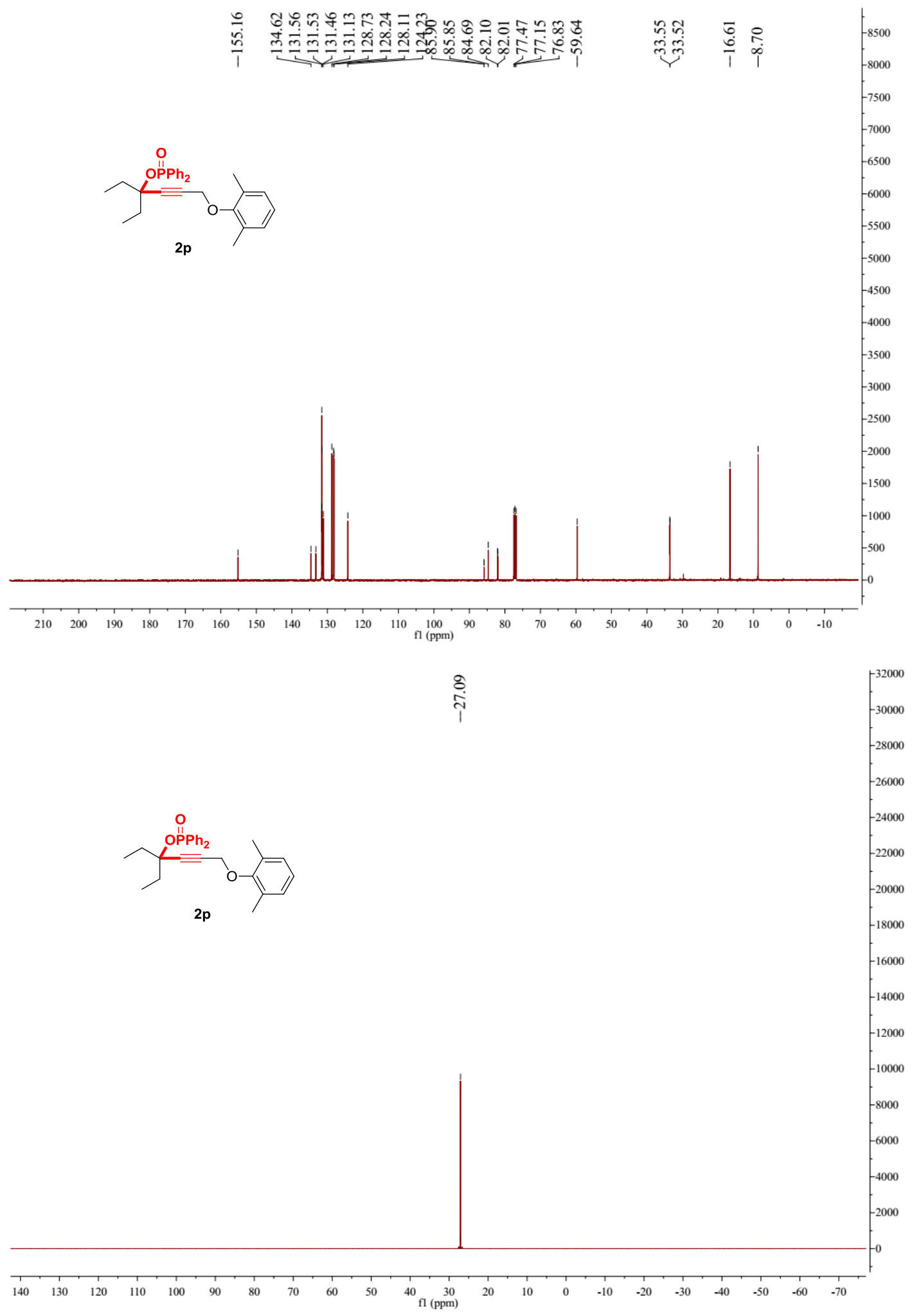


20170505-46_170506160458 \#43-45 RT: 0.40-0.42 AV: 3 NL: 2.01E6

T: FTMS $\{1, \overline{1}\}+p$ ESI Full ms [100.00-1000.00]

$$
\begin{gathered}
219.05684 \\
R=53177
\end{gathered}
$$

$$
\mathrm{z}=1
$$

$\mathrm{C}_{12} \mathrm{H}_{12} \mathrm{O}_{2} \mathrm{P}=219.05694$
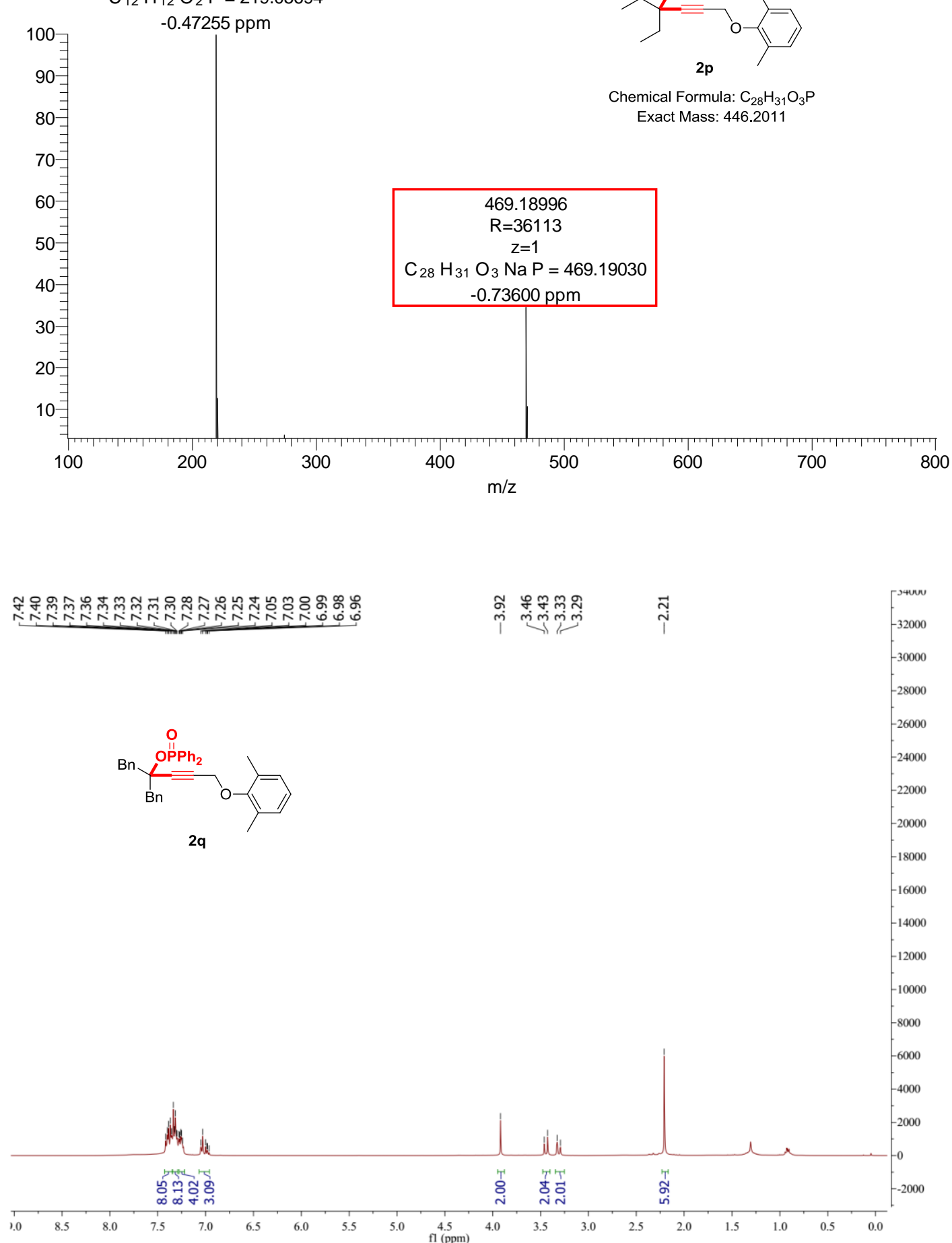

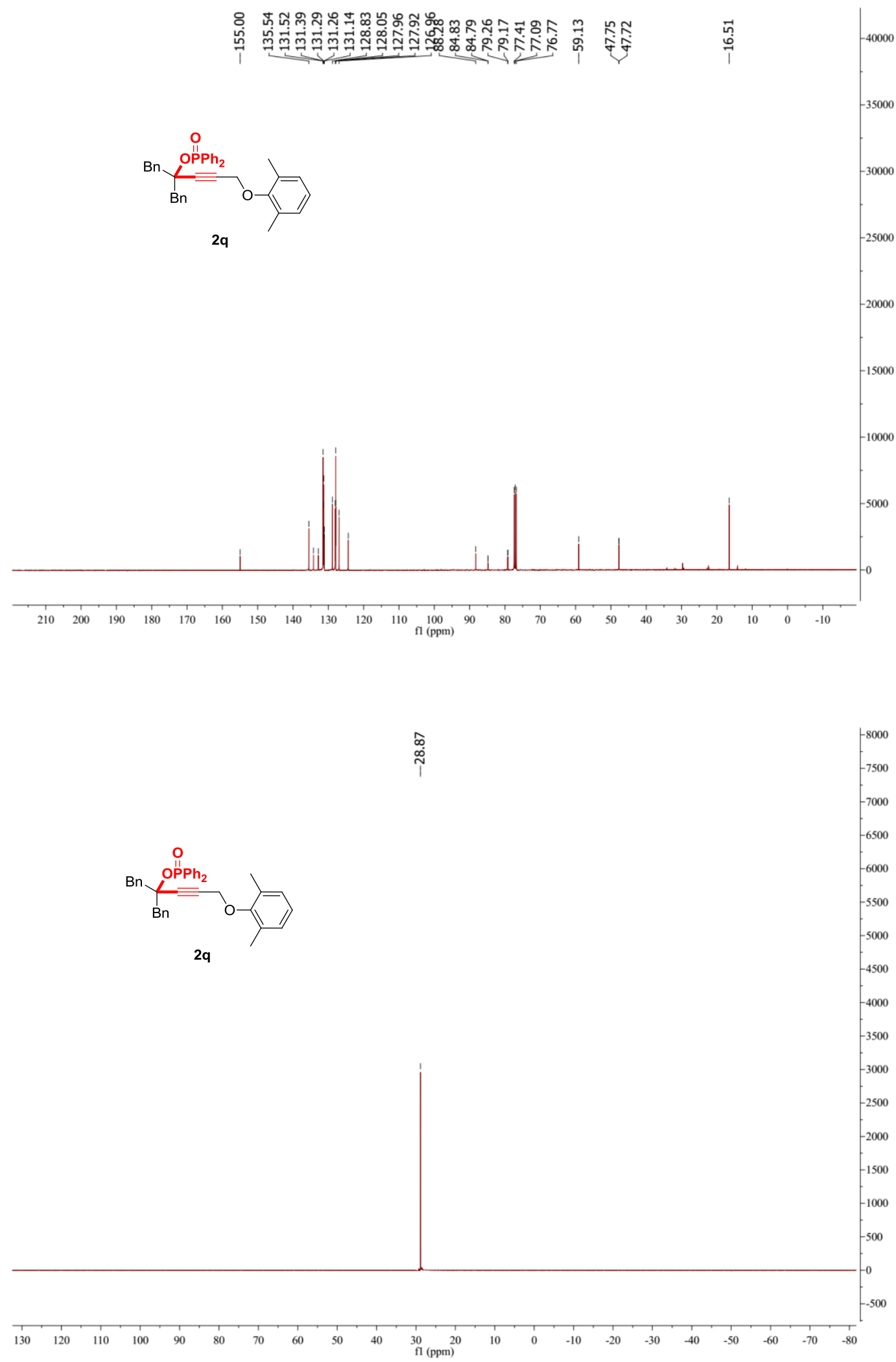
NJNY-20180326-3 \#17 RT: 0.25 AV: 1 NL: 8.20E6

T: FTMS $\{1,1\}+p$ ESI Full ms [100.00-1000.00]

\begin{tabular}{|c|}
593.22131 \\
$\mathrm{R}=33100$ \\
$\mathrm{Z}=1$ \\
$\mathrm{C}_{38} \mathrm{H}_{35} \mathrm{O}_{3} \mathrm{Na} \mathrm{P}=593.22160$ \\
$-0.48746 \mathrm{ppm}$ \\
\hline
\end{tabular}
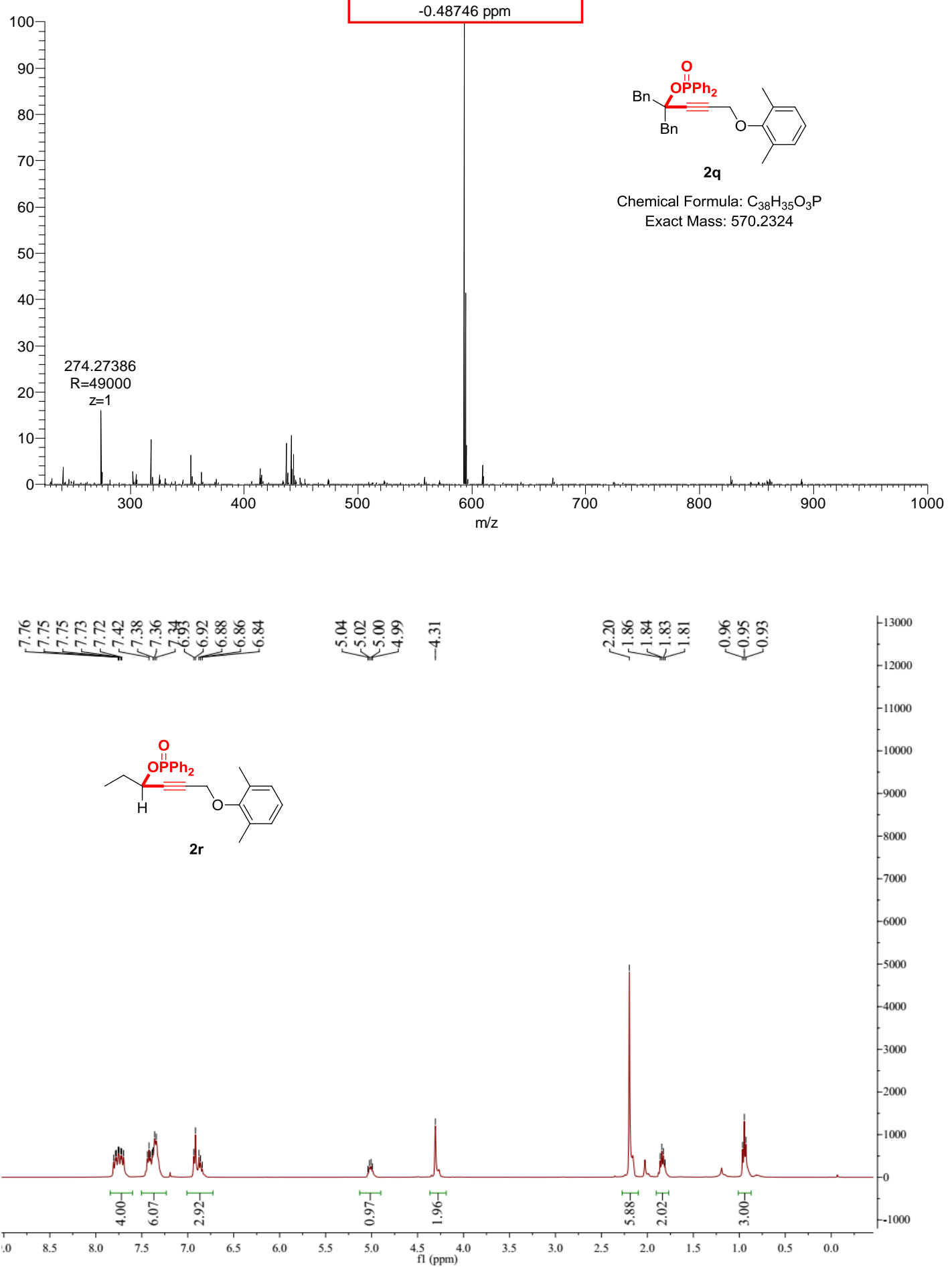

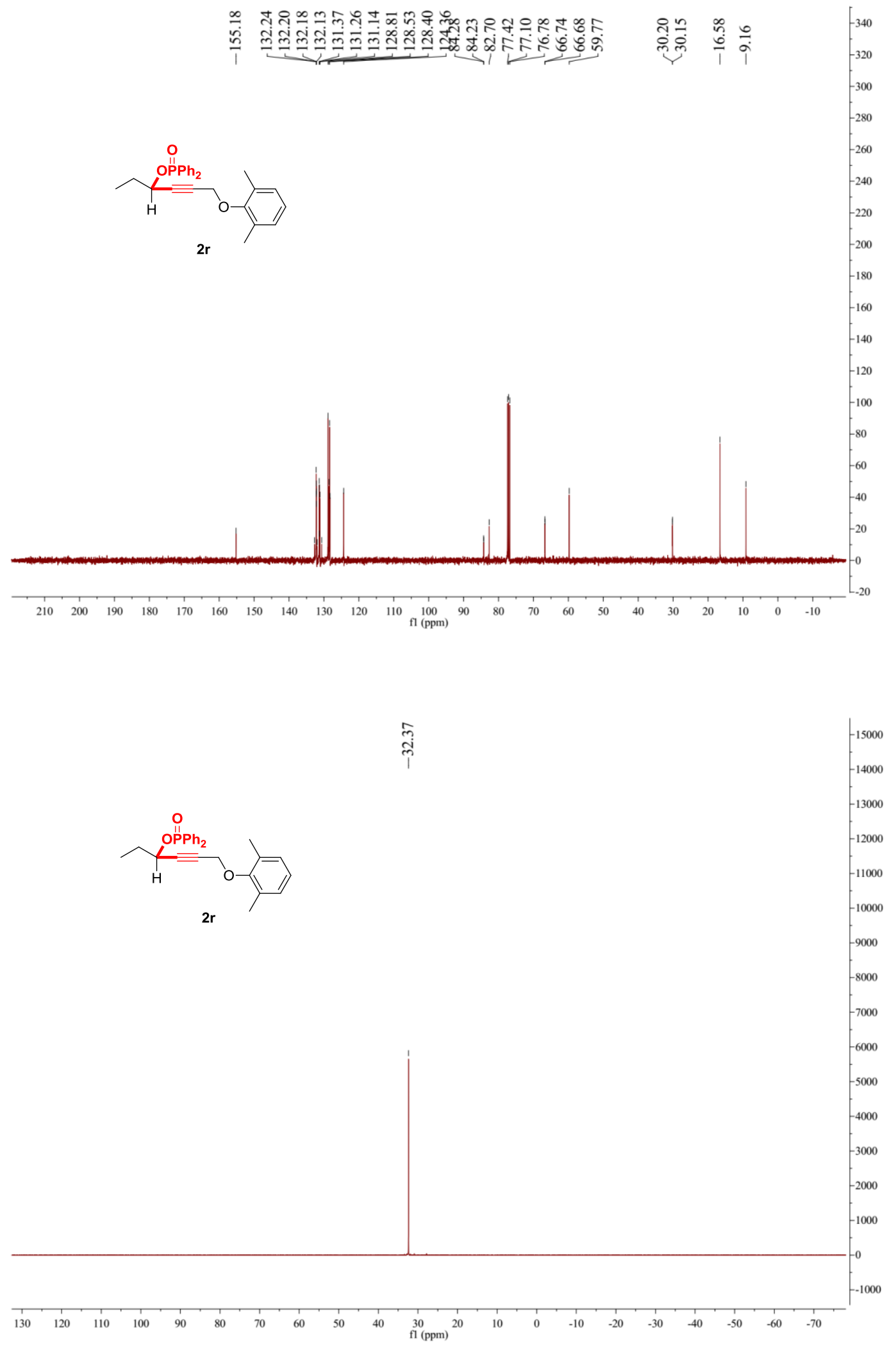
20170714-30 \#61-62 RT: 0.52-0.53 AV: 2 NL: 1.08E6

T: FTMS $\{1,1\}+$ p ESI Full ms [100.00-1000.00]
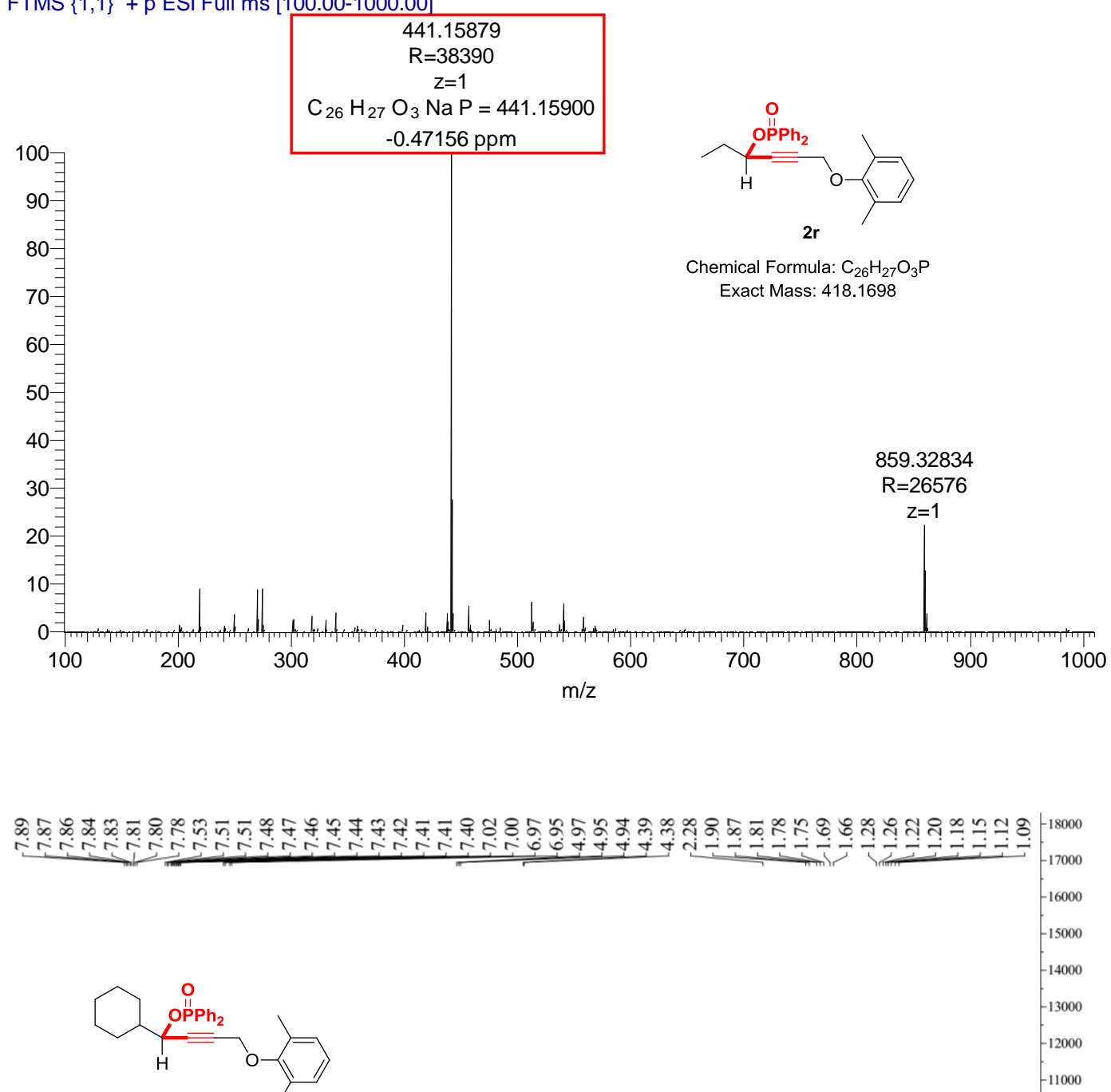

2s

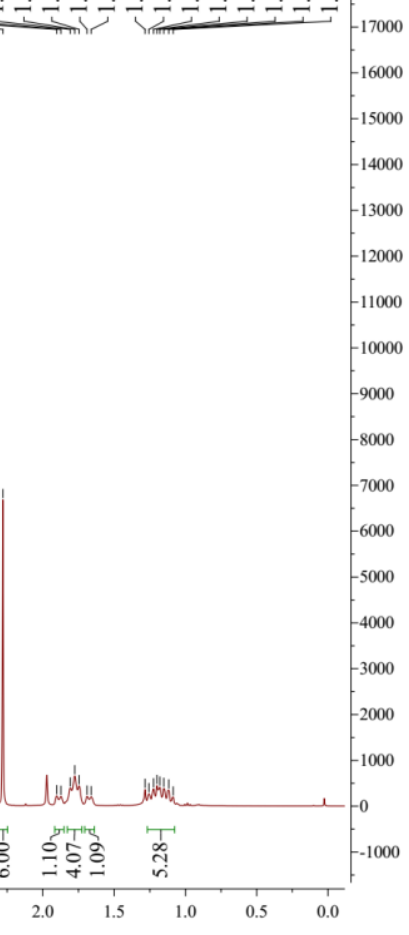



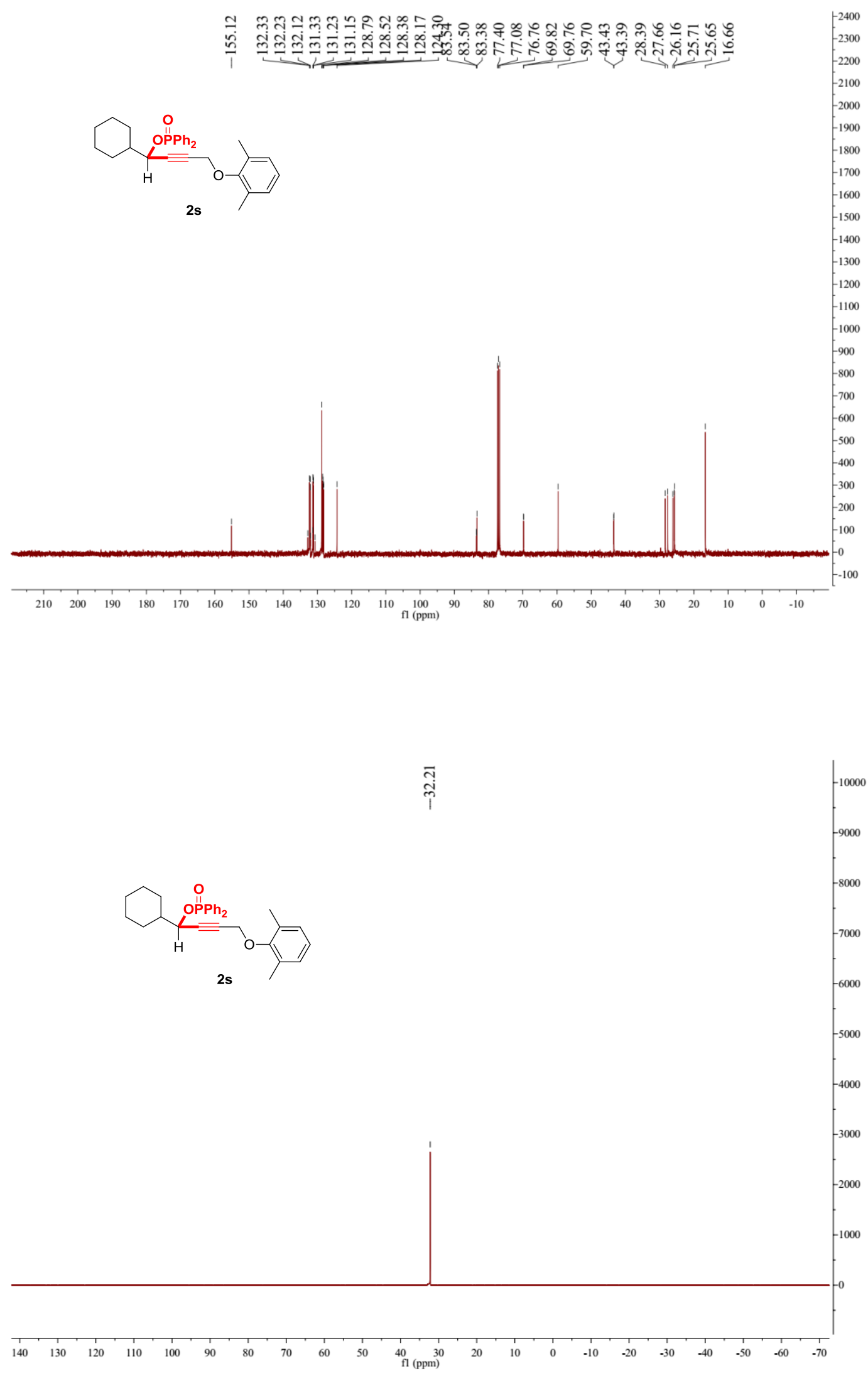
20170714-29 \#57-59 RT: 0.48-0.50 AV: 3 NL: $1.03 E 6$

T: FTMS $\{1,1\}+p$ ESI Full ms [100.00-1000.00]

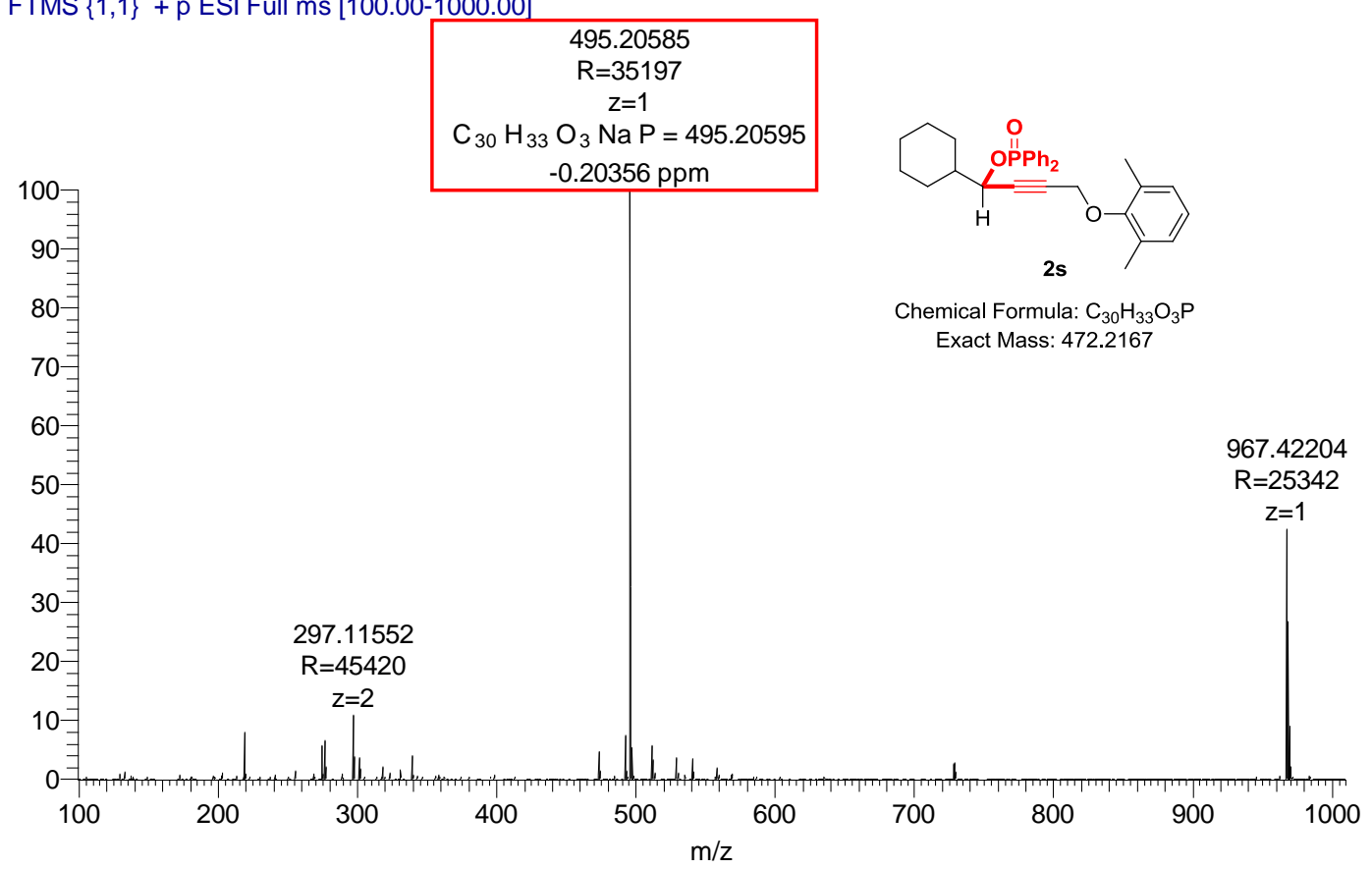

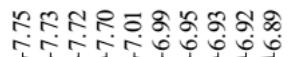

†

$-42000$

1,

$\mathrm{MeO}$

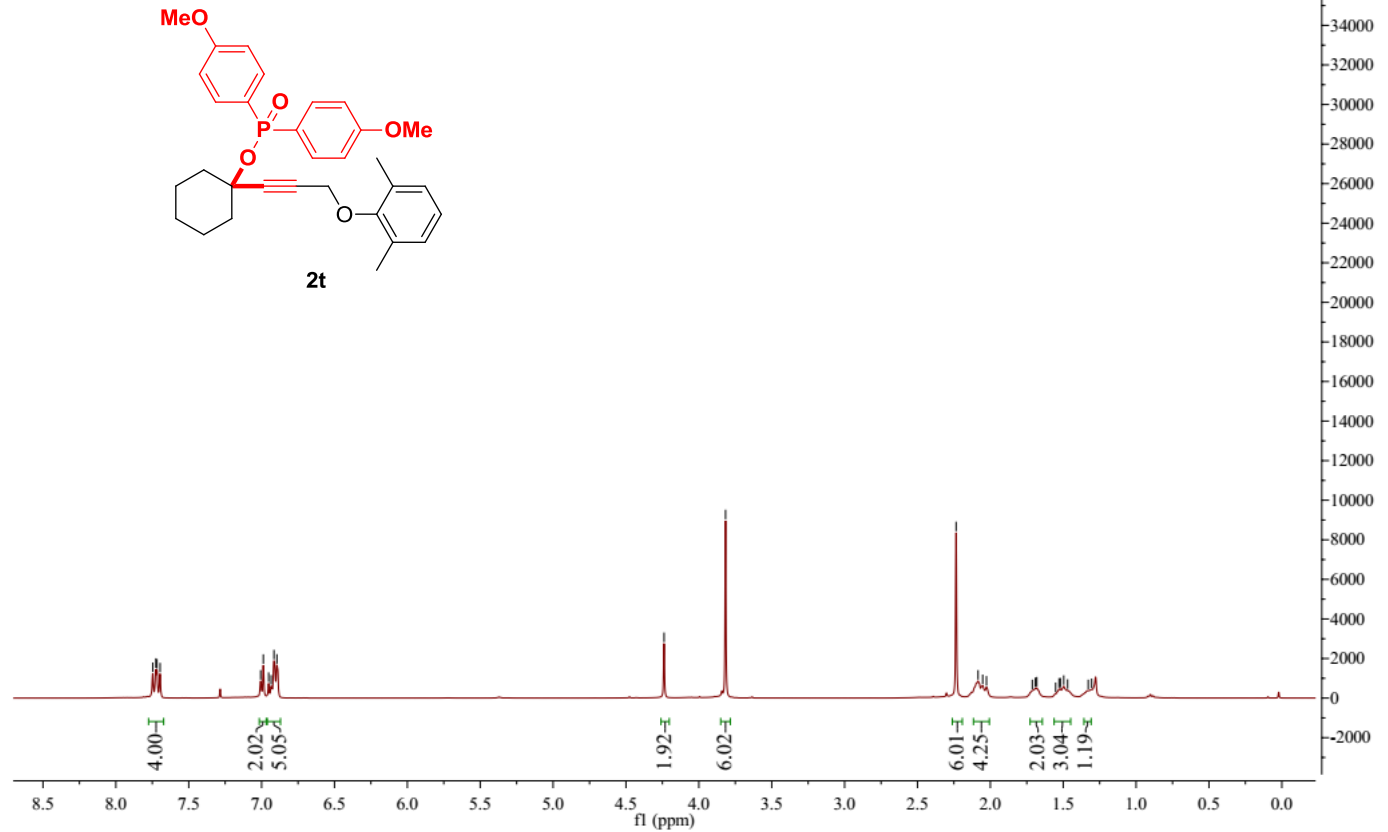

25342 

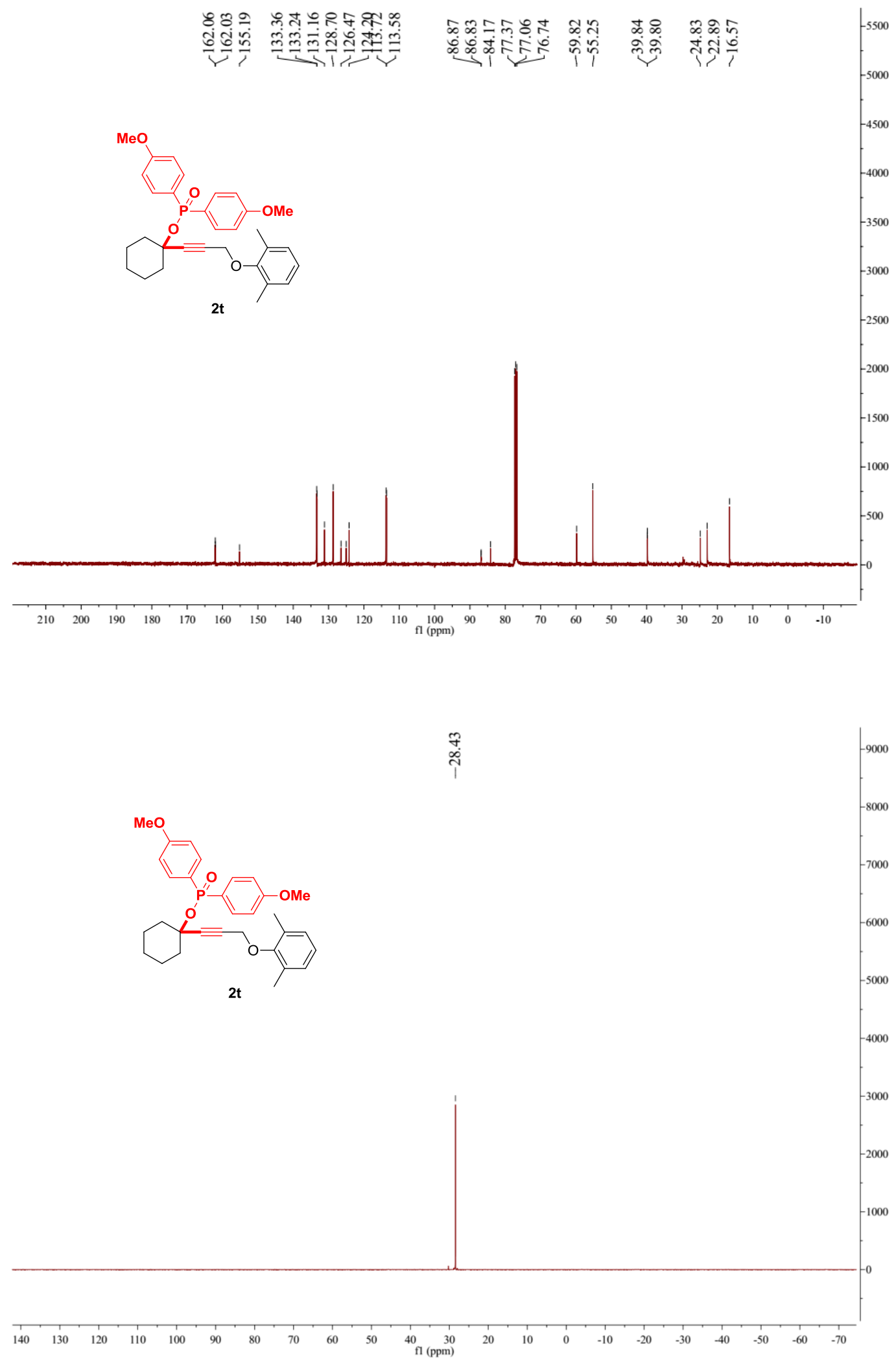
20170618-7 \#56 RT: 0.52 AV: 1 NL: 4.10E5

T: FTMS $\{1,1\}+p$ ESI Full ms [100.00-1000.00]

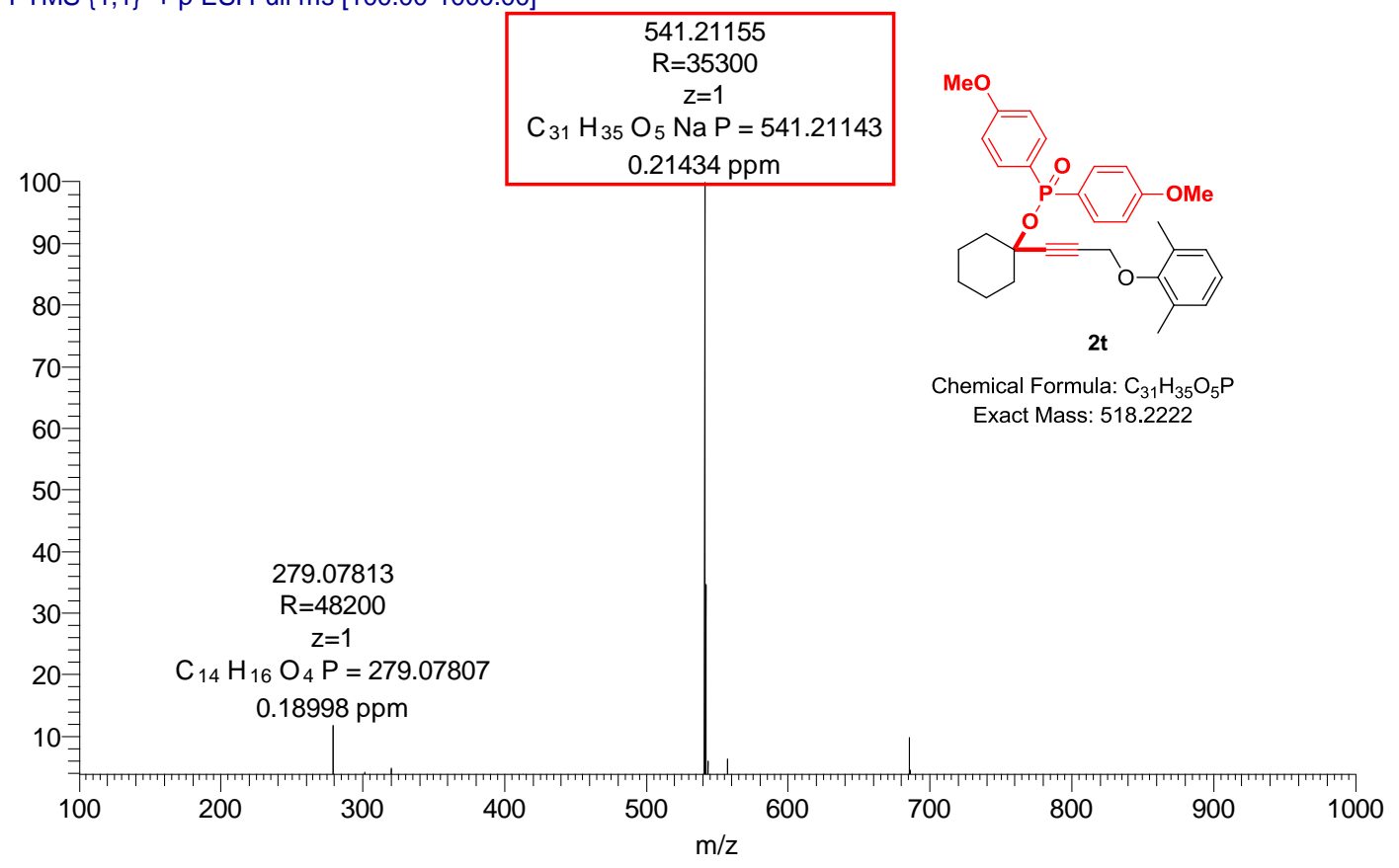

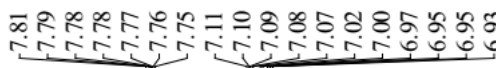

$\underset{d}{7}$

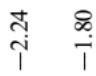
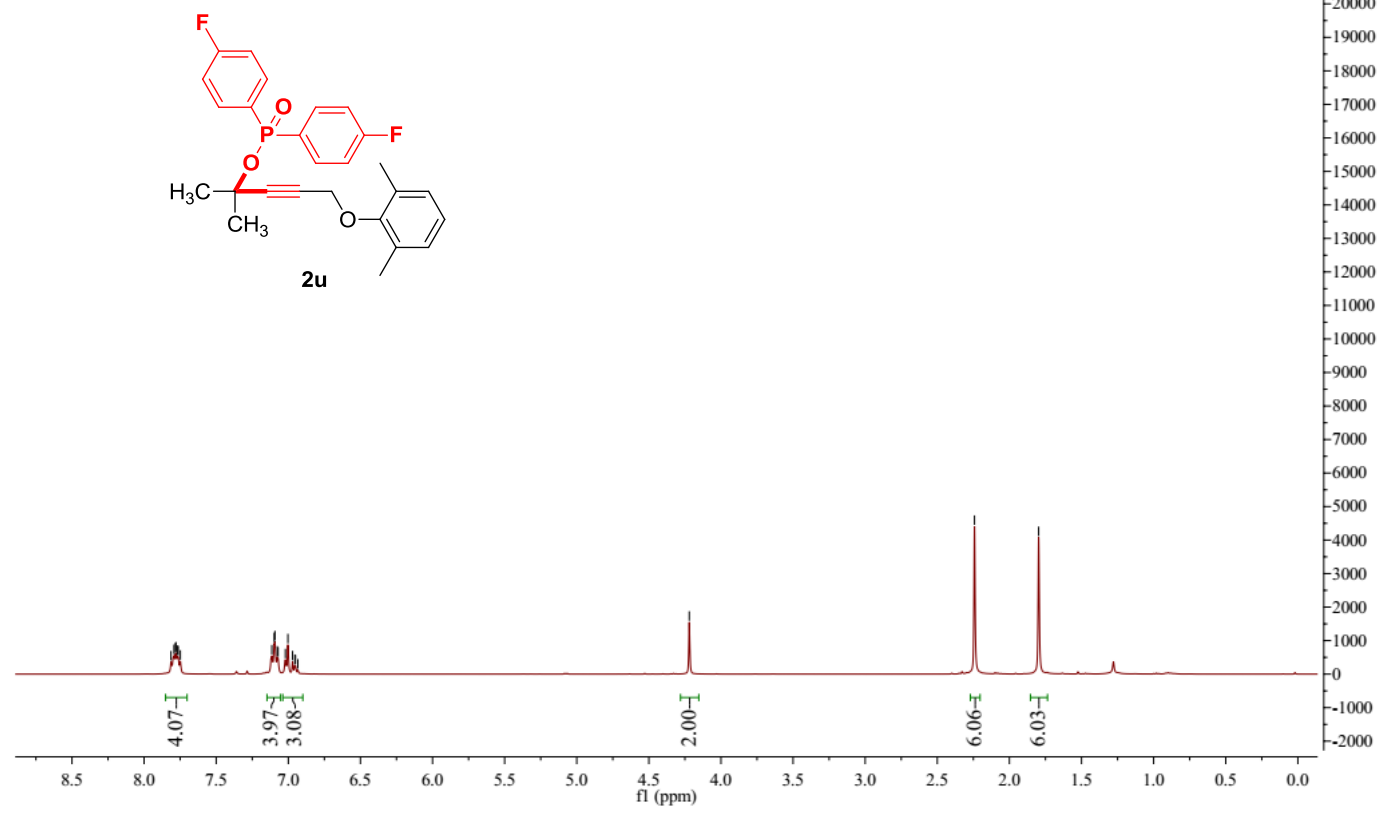

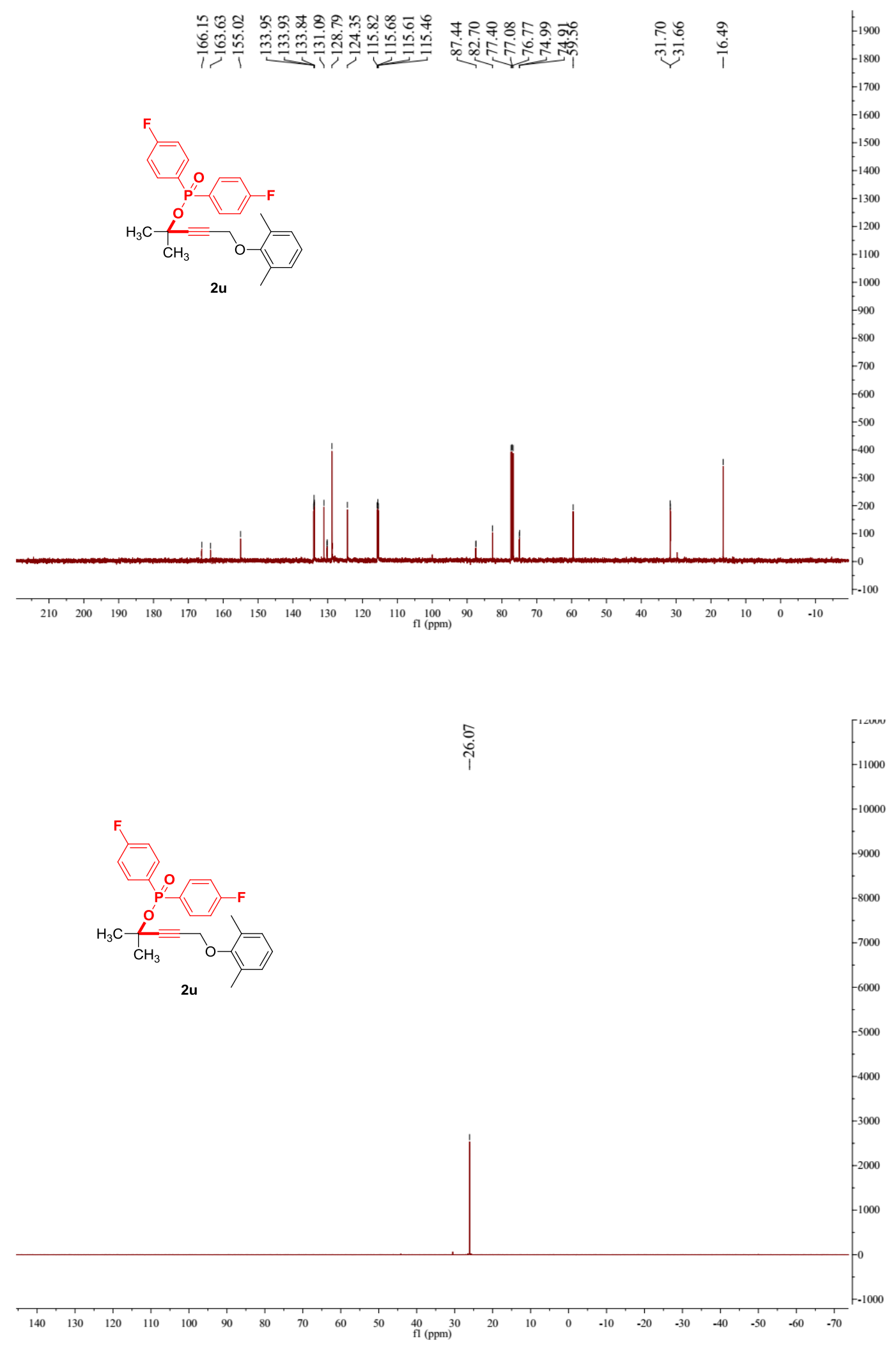
20171124-23 \#21-23 RT: 0.21-0.22 AV: 3 NL: 1.17E7

T: FTMS $\{1,1\}+p$ ESI Full ms [100.00-1000.00]

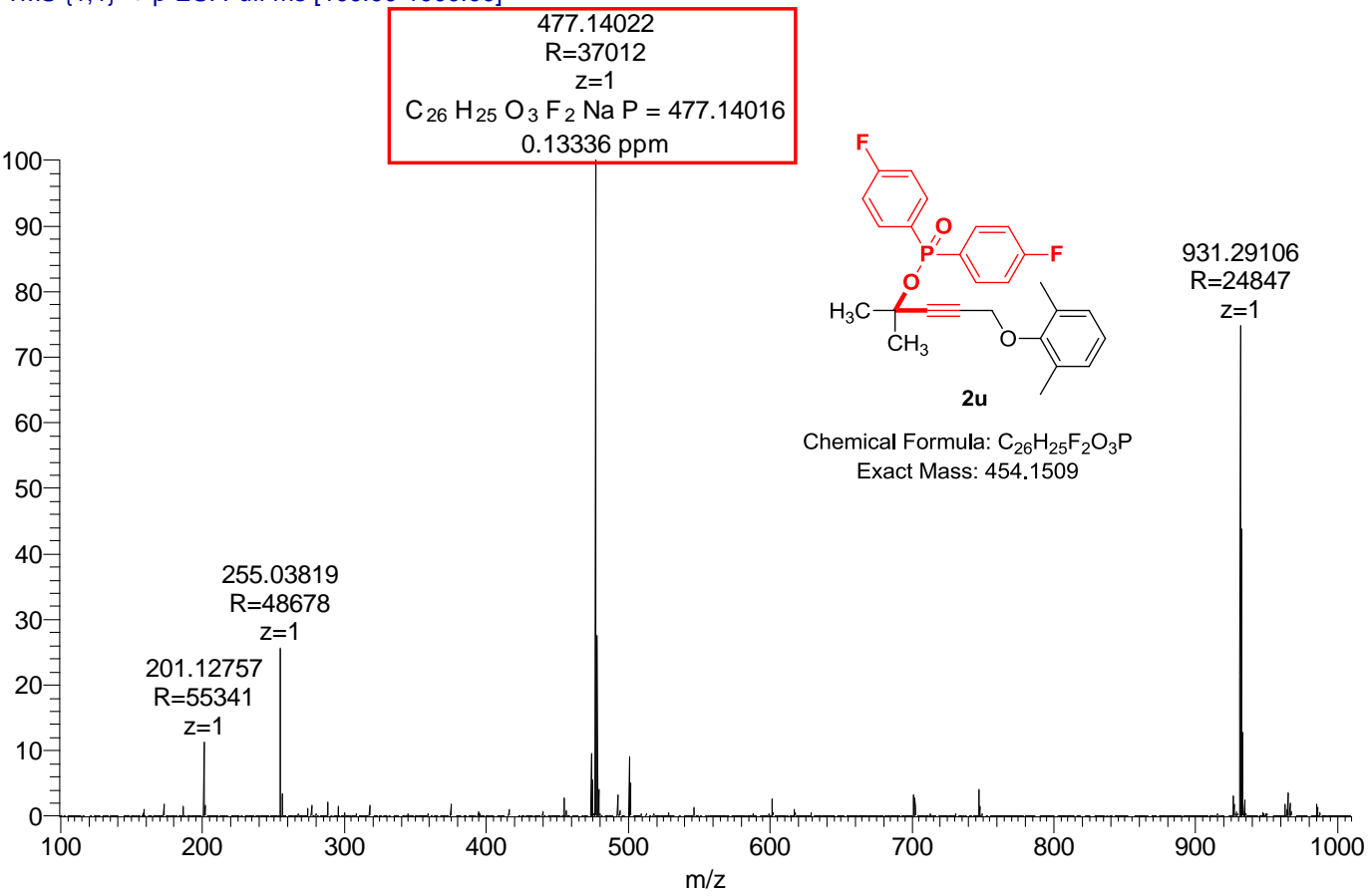

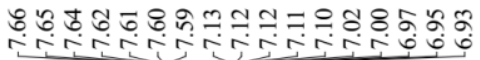

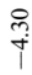

$\stackrel{\text { i⿱ }}{\stackrel{\infty}{i}}$
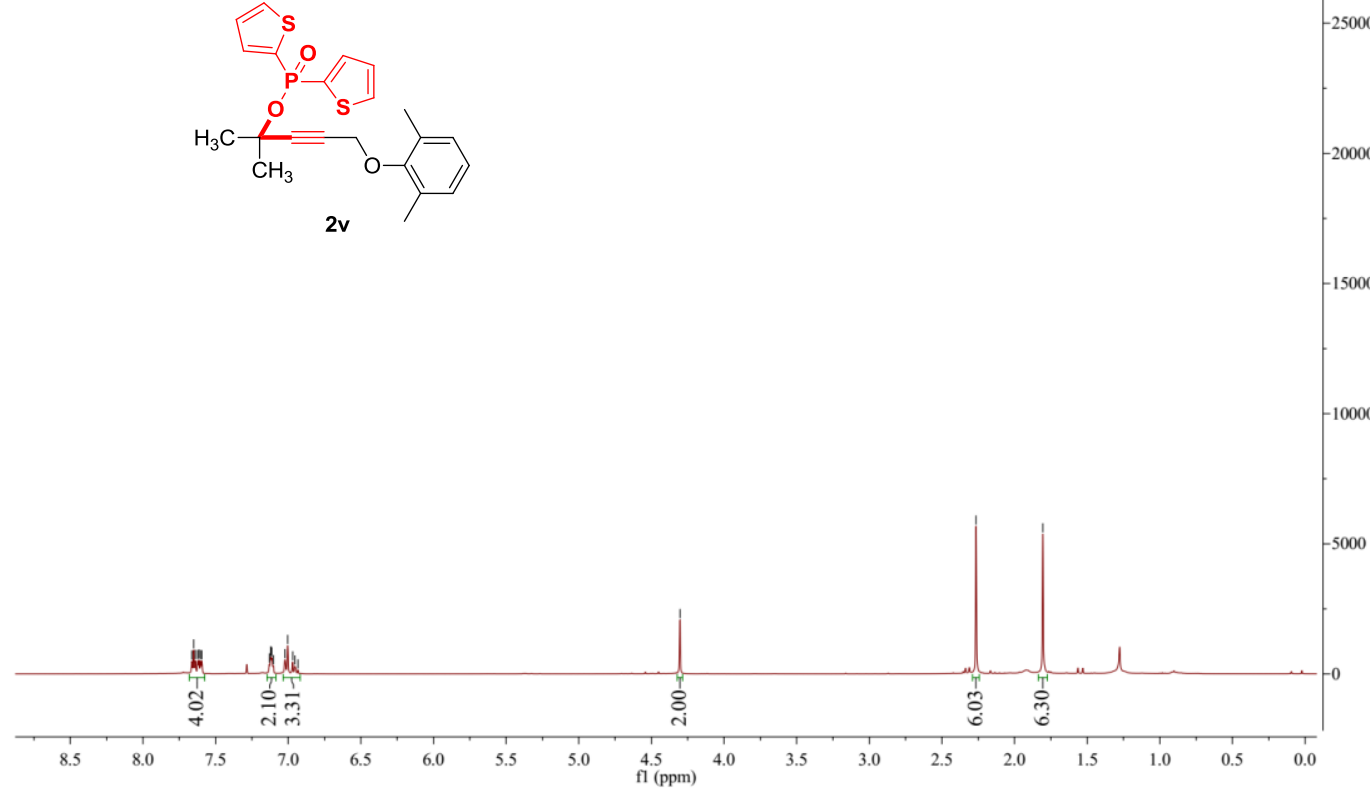

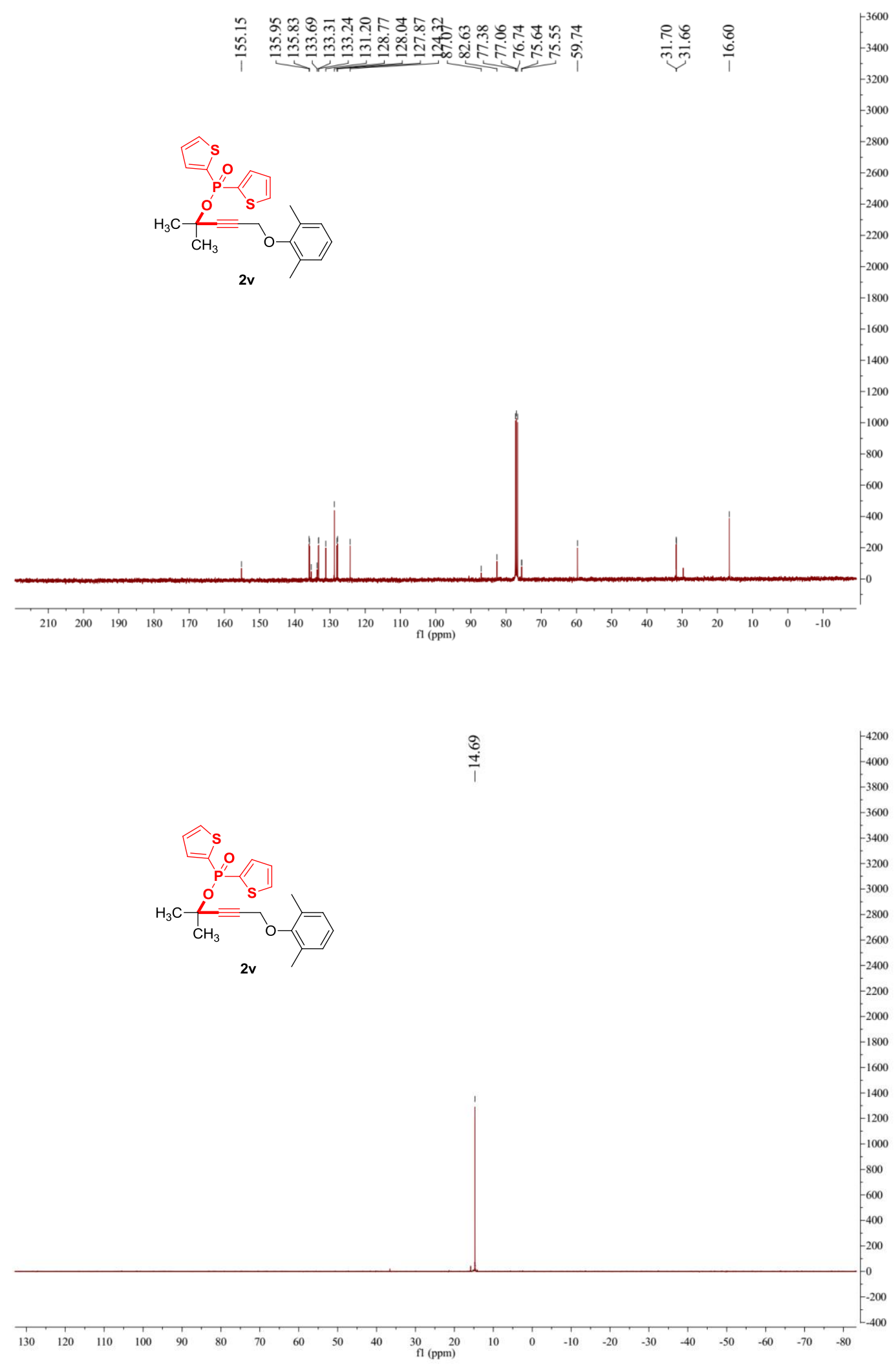
20170618-8 \#50-53 RT: 0.45-0.48 AV: 4 NL: 3.97E5

T: FTMS $\{1,1\}+p$ ESI Full ms $[100.00-1000.00]$

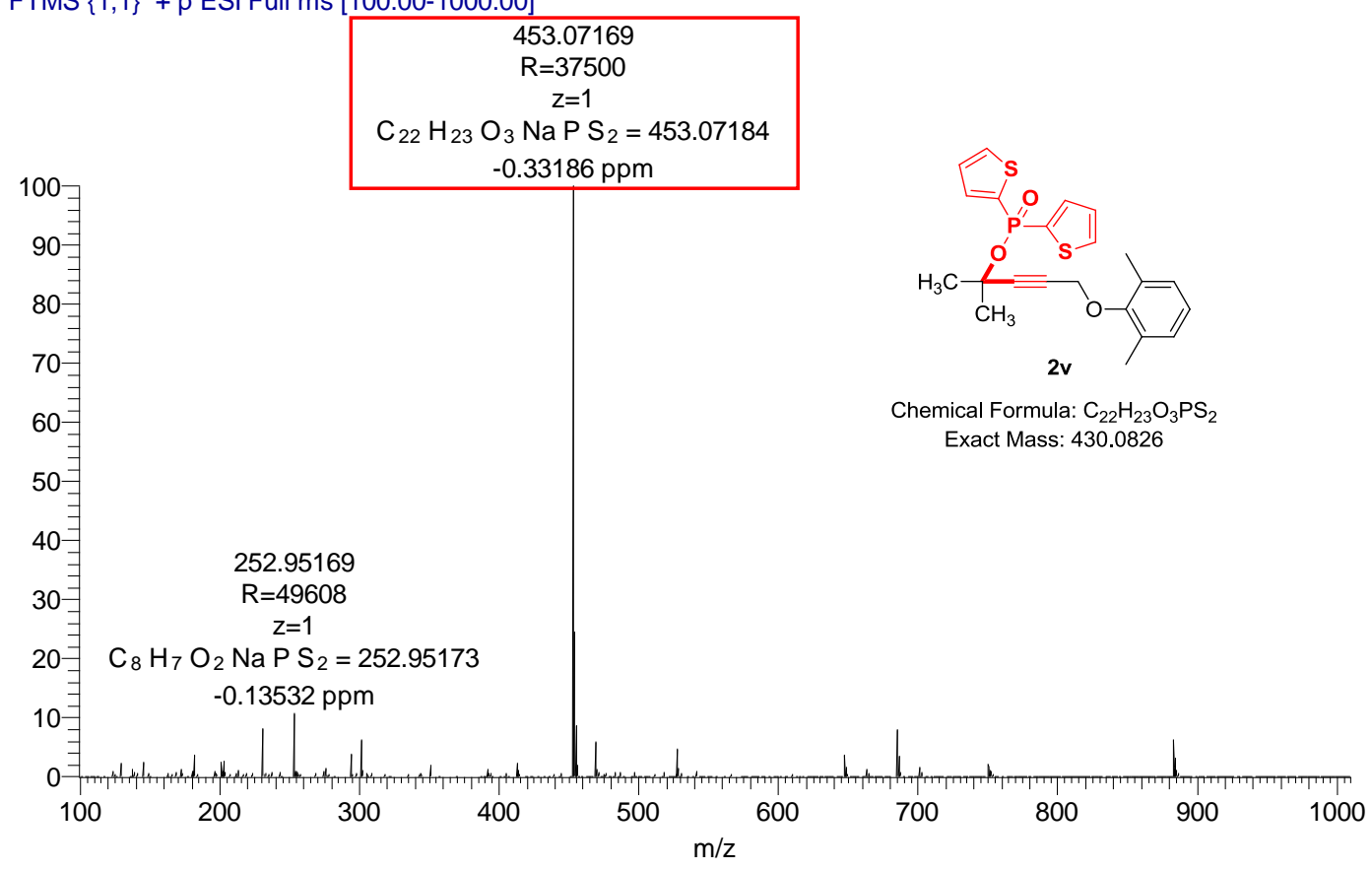

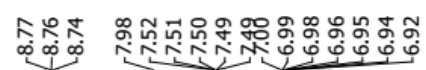

$\underset{+}{\tilde{T}} \quad \stackrel{n}{i} \underset{i}{\infty}$

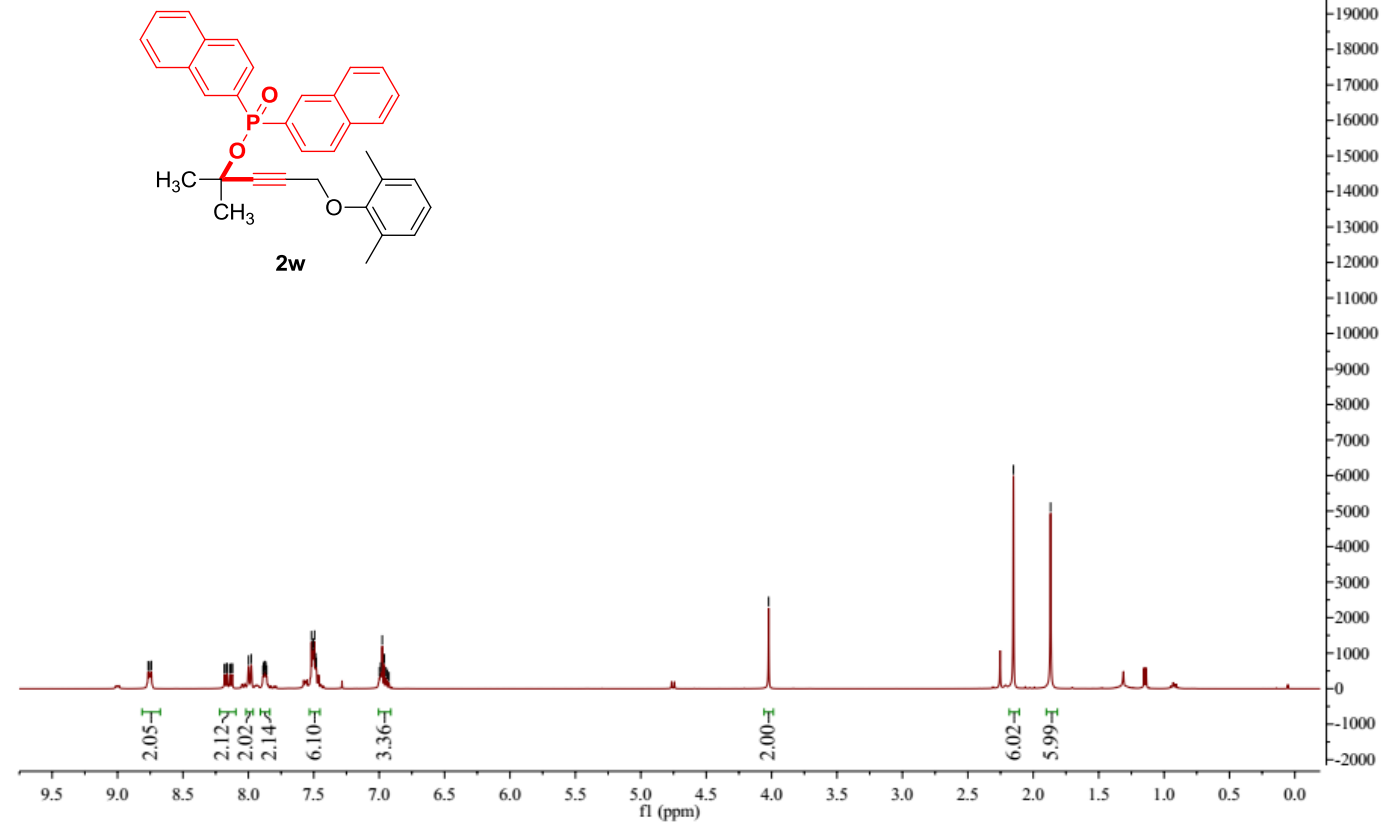

$-23000$ 


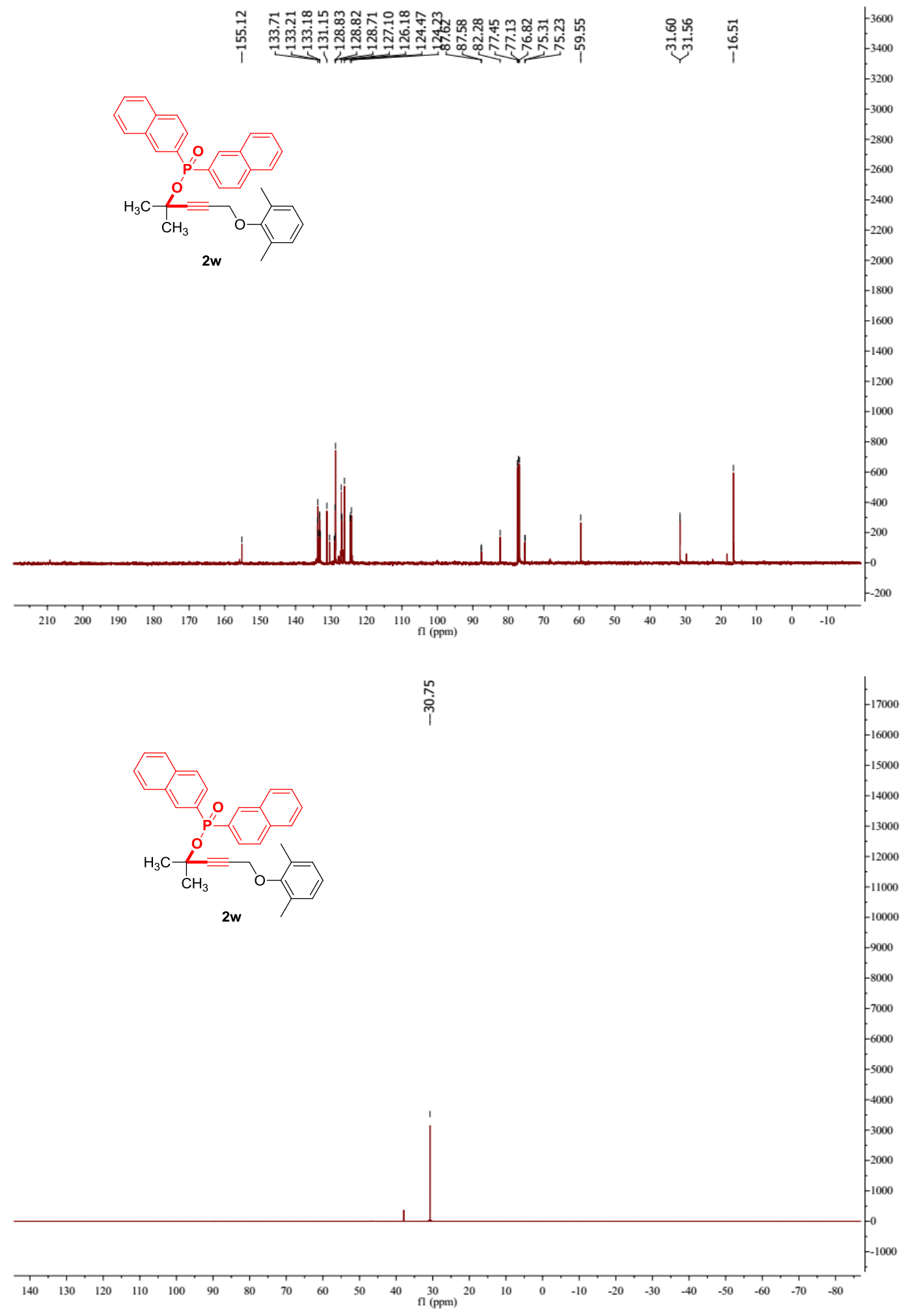


20181115-24 \#39 RT: 0.58 AV: 1 SB: 3 0.01-0.04 NL: 3.64E5 T: FTMS $\{1,1\}+p$ ESI Full ms [100.00-1000.00]

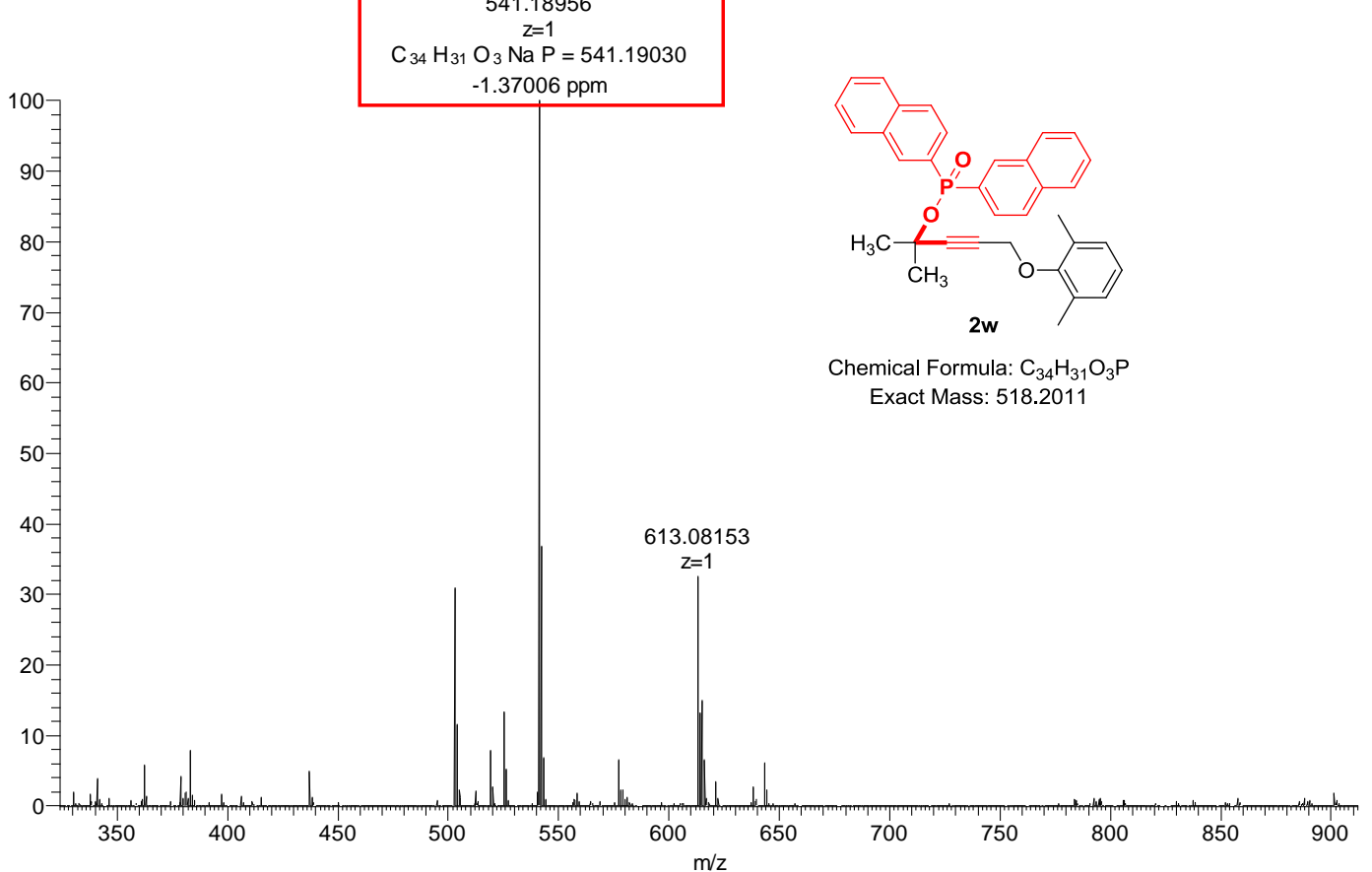

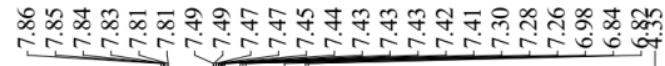

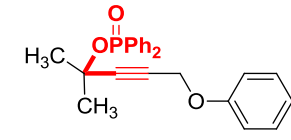

2x
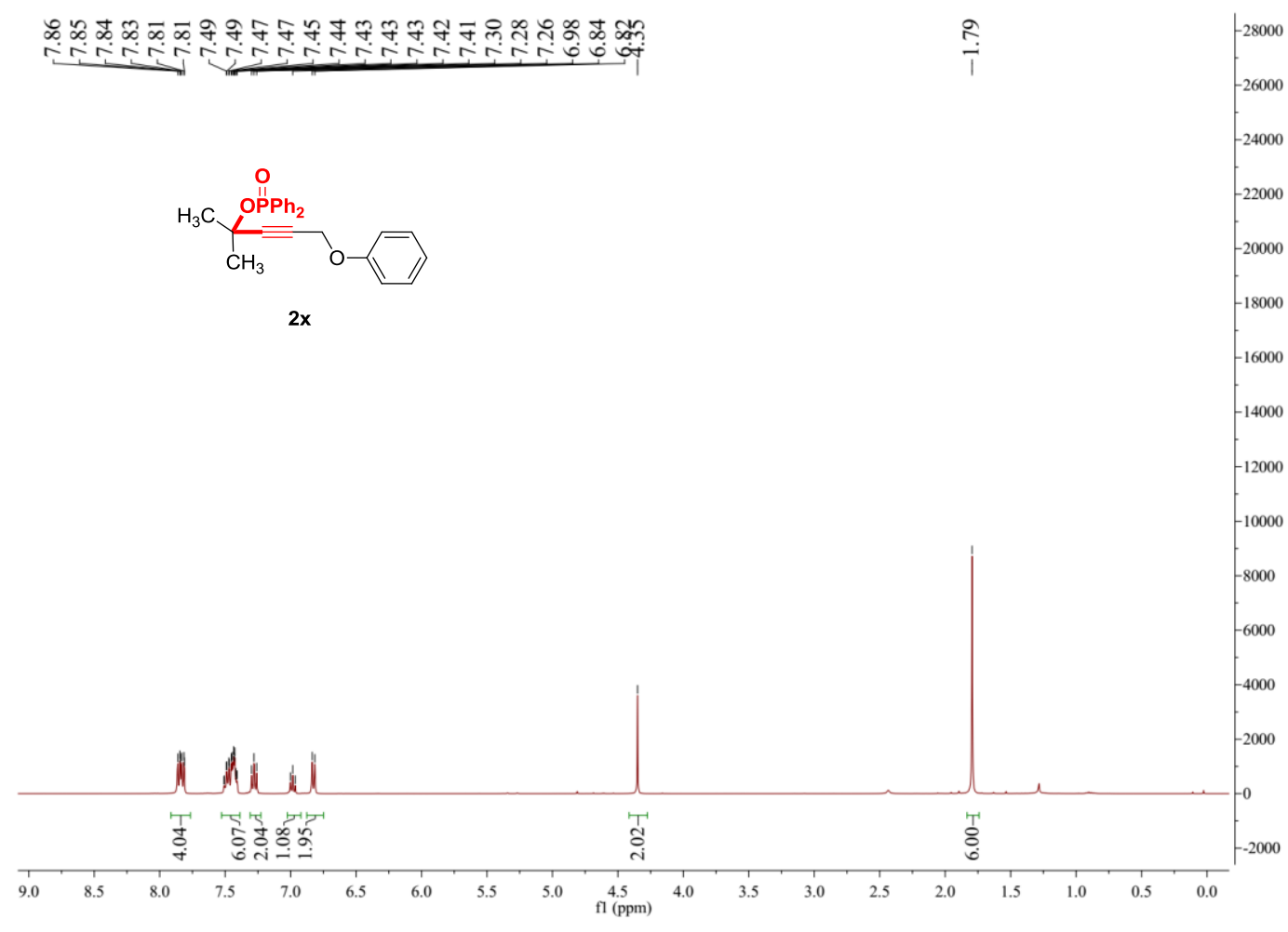

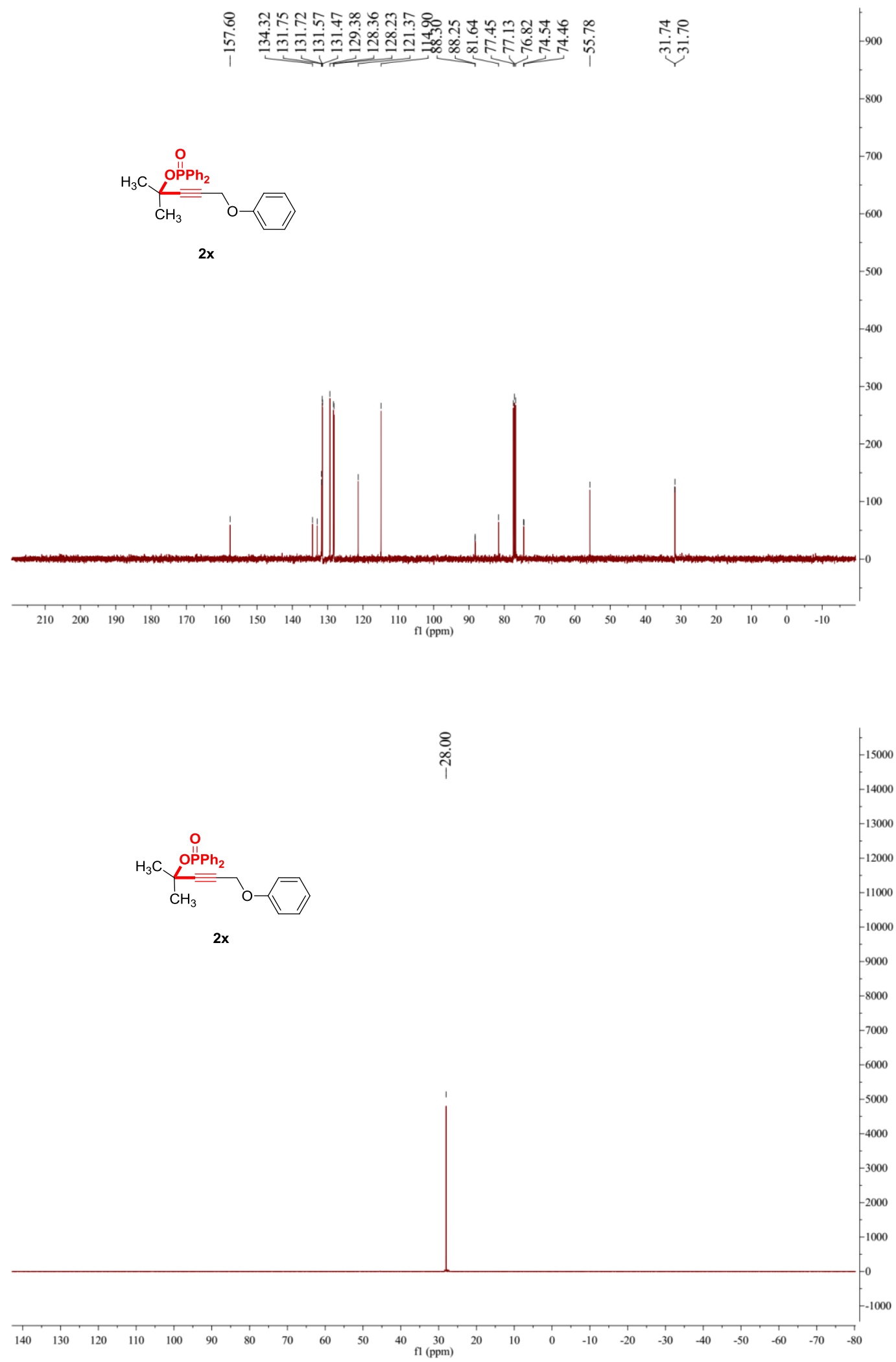
20171124-22 \#43 RT: 0.38 AV: 1 NL: 3.02E6

T: FTMS $\{1,1\}+$ p ESI Full ms [100.00-1000.00]

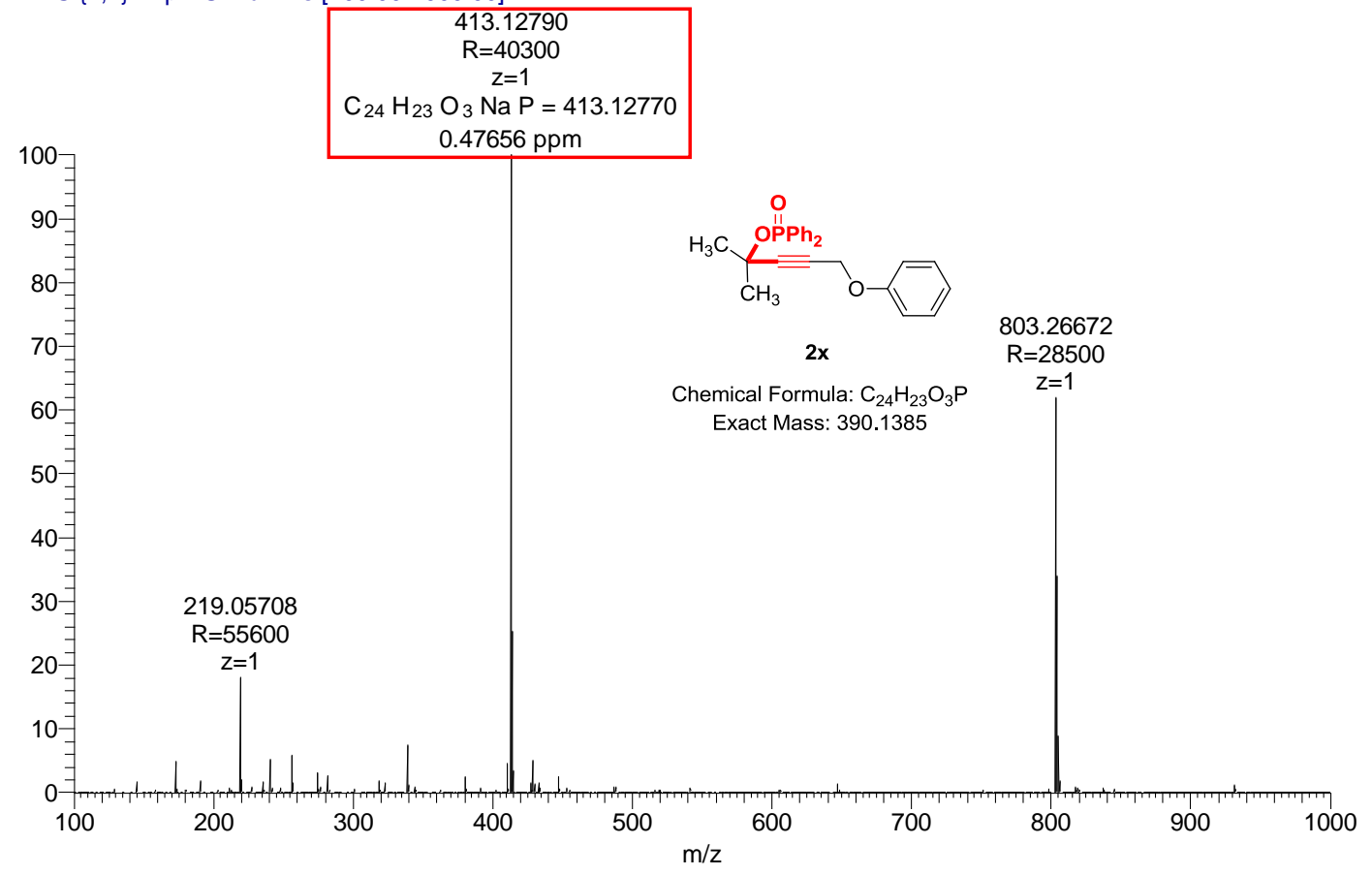

$\underbrace{0}$

तิ
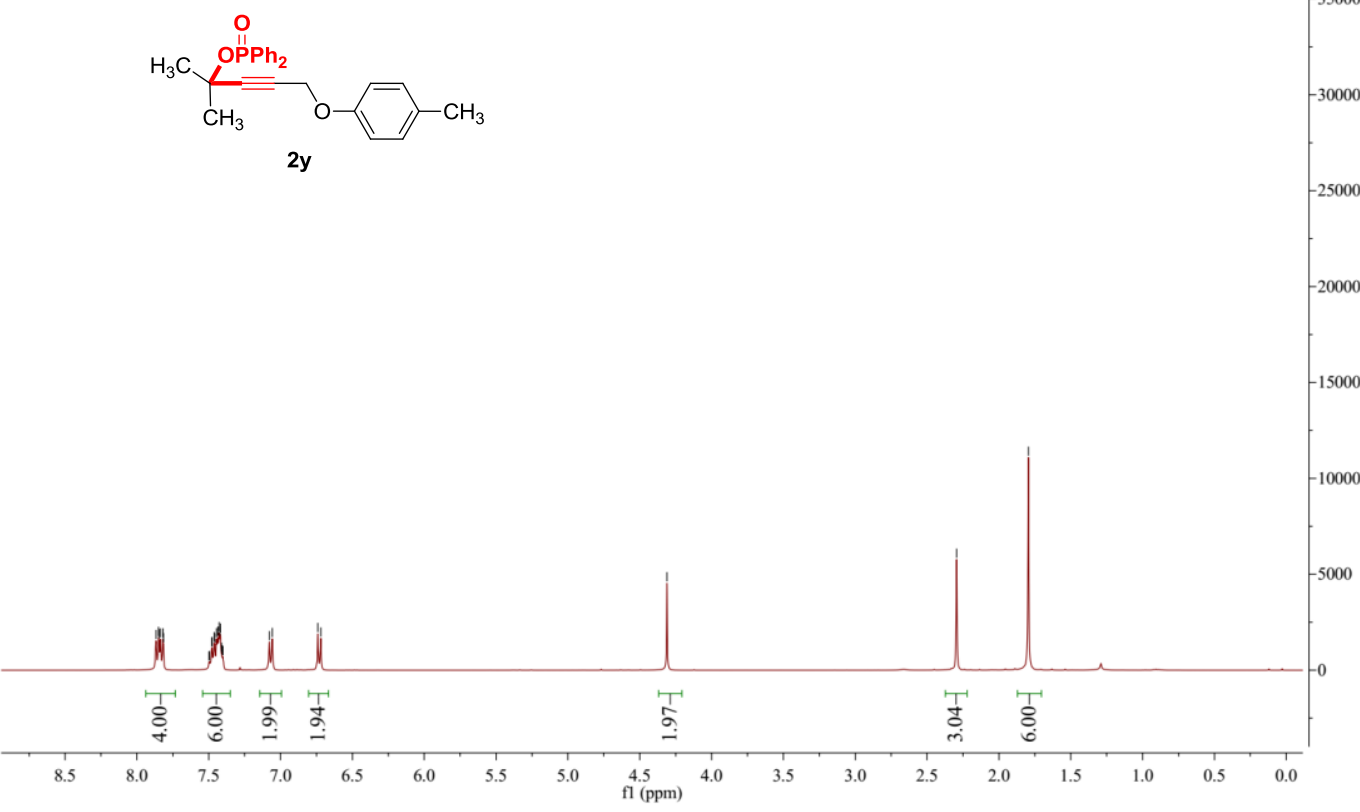


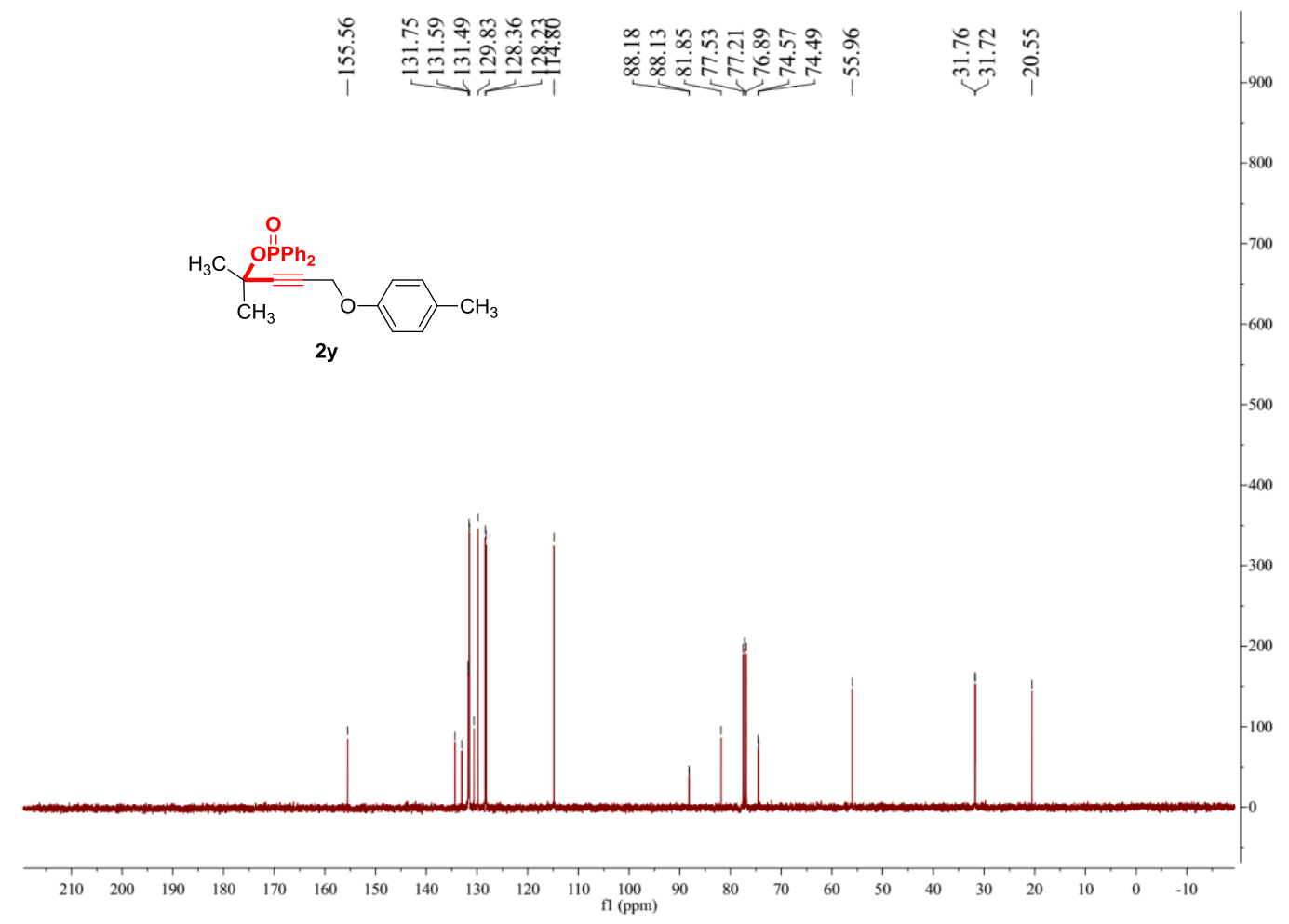

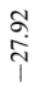
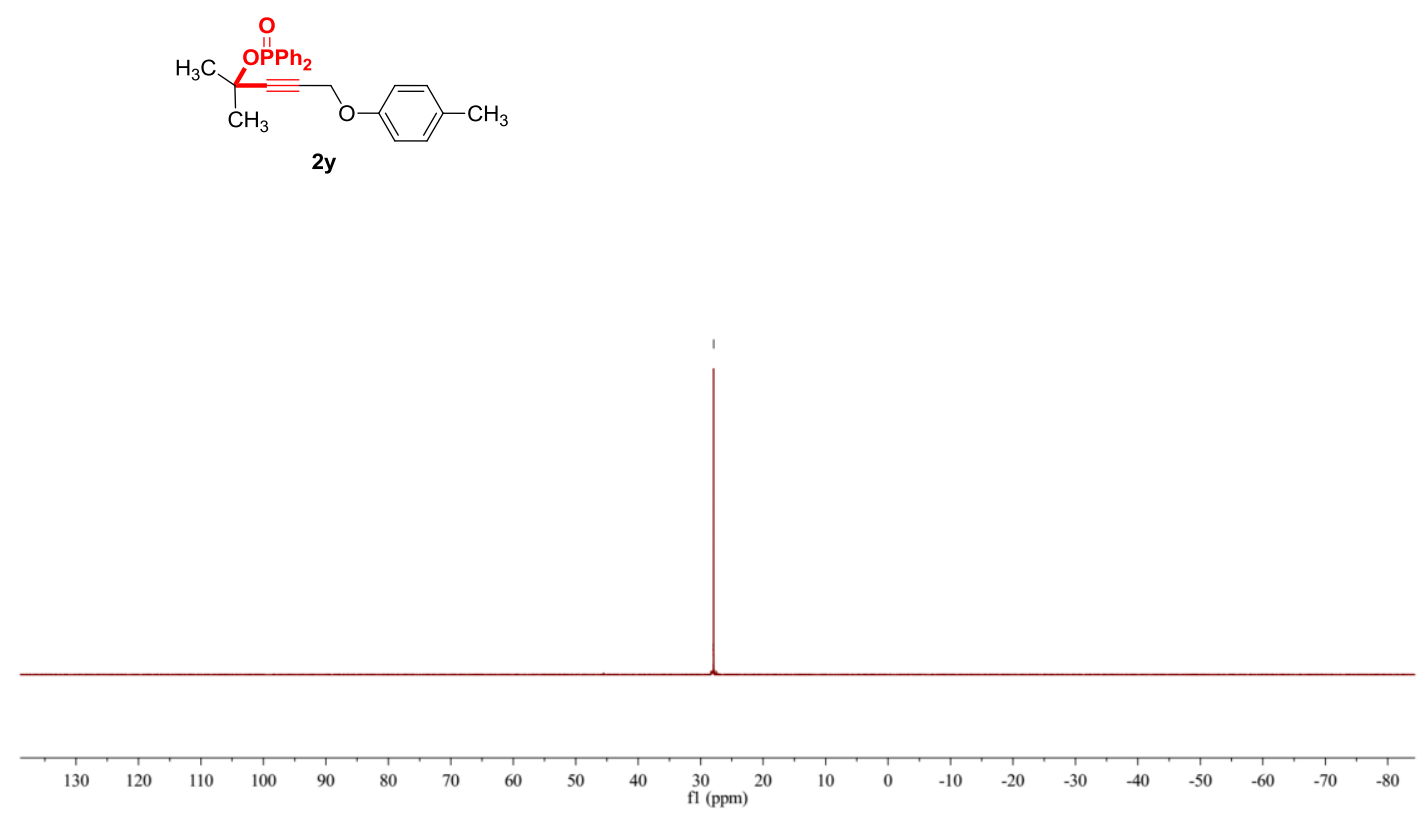
20171124-21 \#45-47 RT: 0.39-0.40 AV: 3 NL: 3.19E6

T: FTMS $\{1,1\}$ + p ESI Full ms [100.00-1000.00]

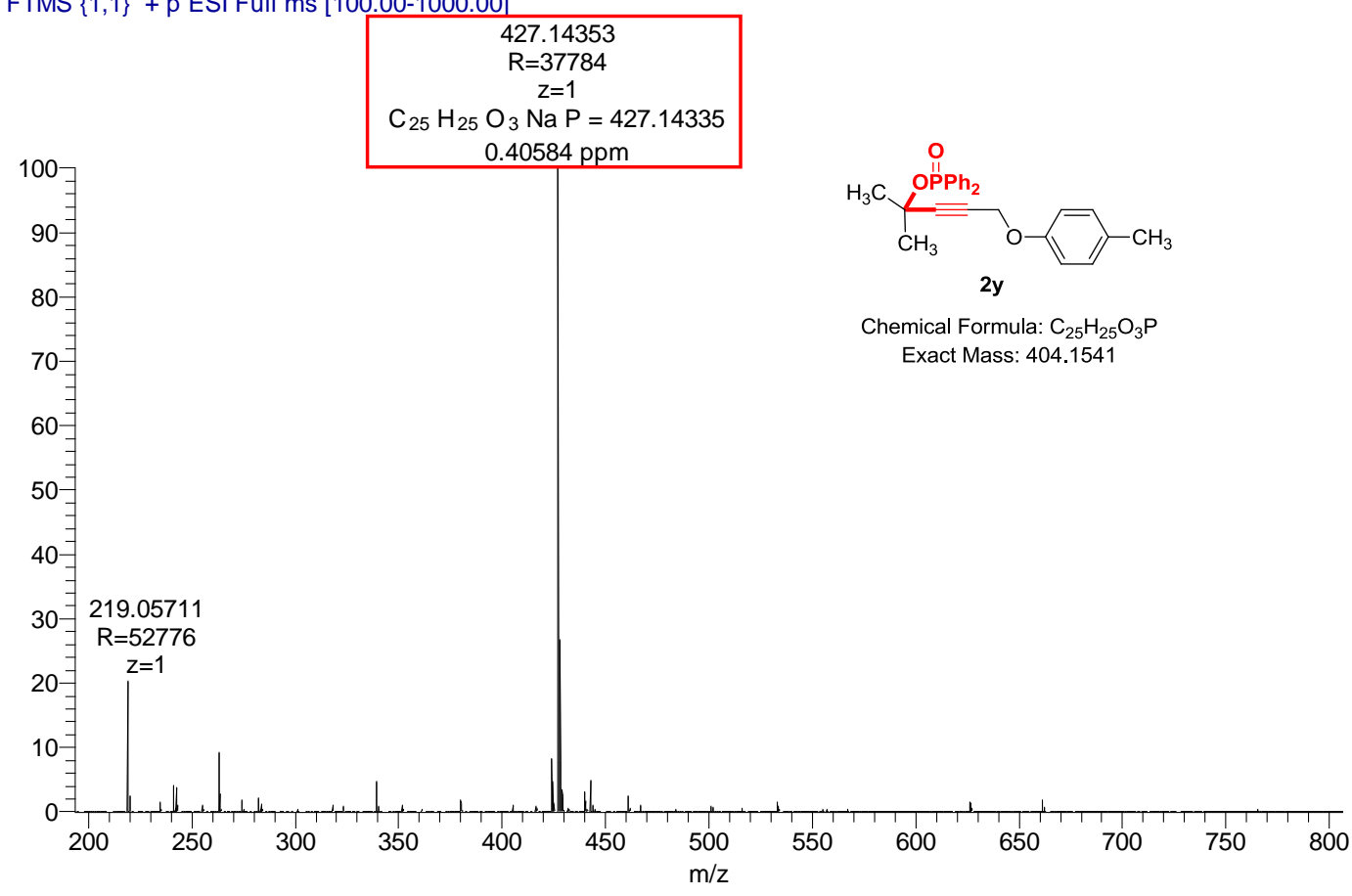

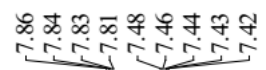

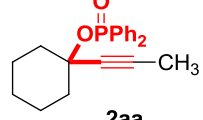

2aa

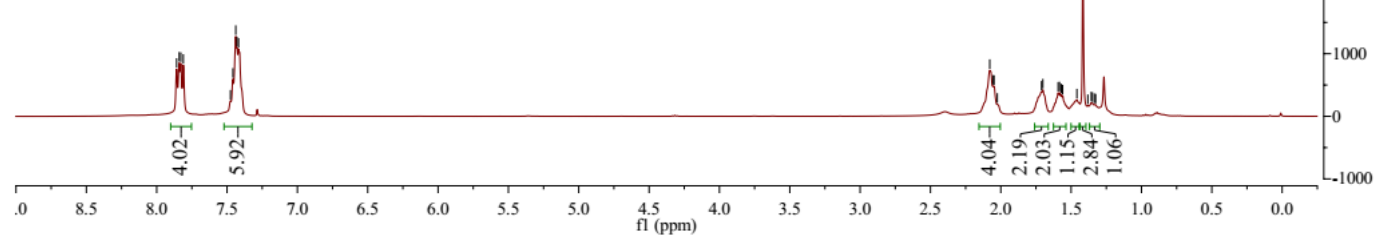



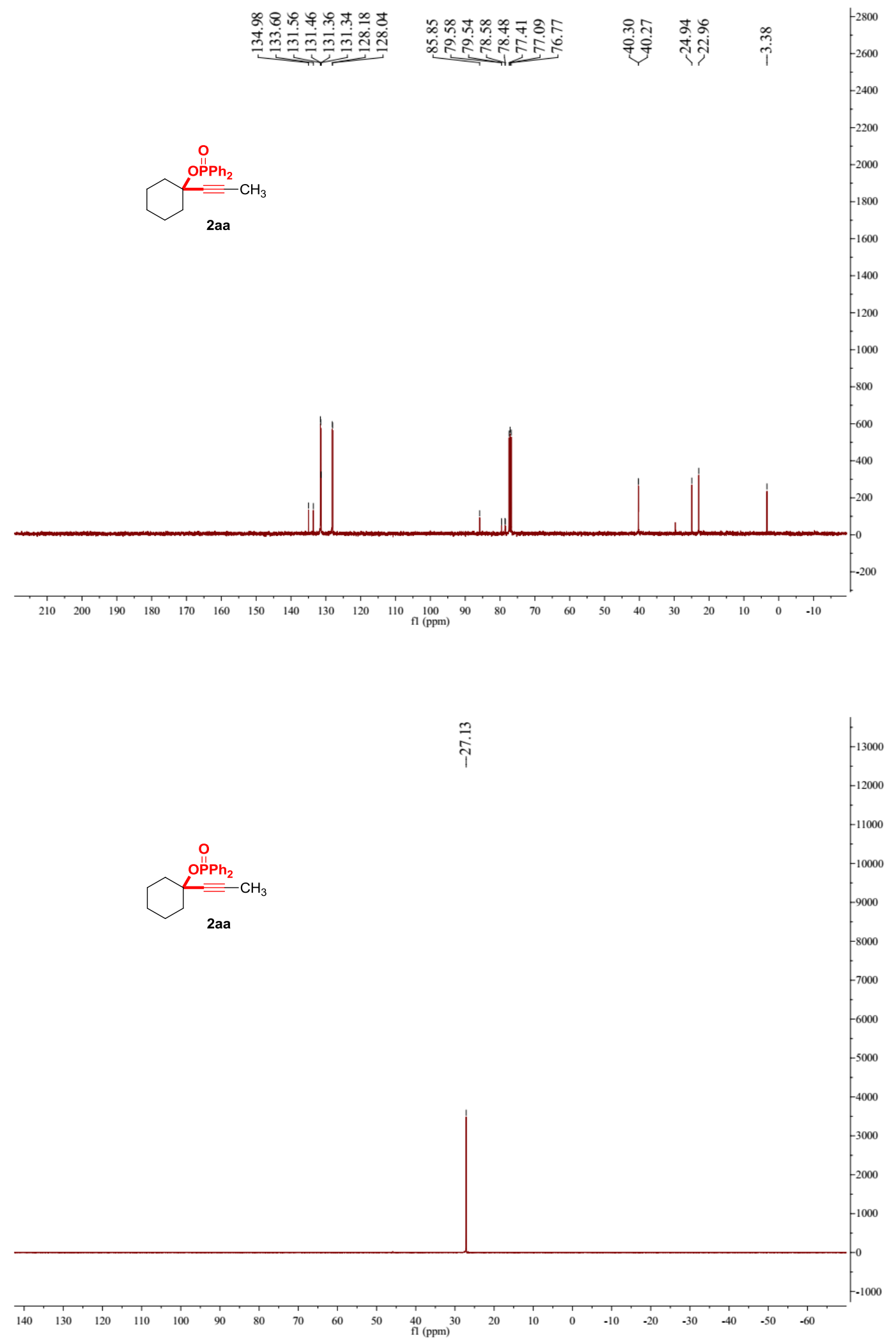
20171124-20 \#47-49 RT: 0.39-0.41 AV: 3 NL: 9.30E5 T: FTMS $\{1,1\}+$ p ESI Full ms [100.00-1000.00]
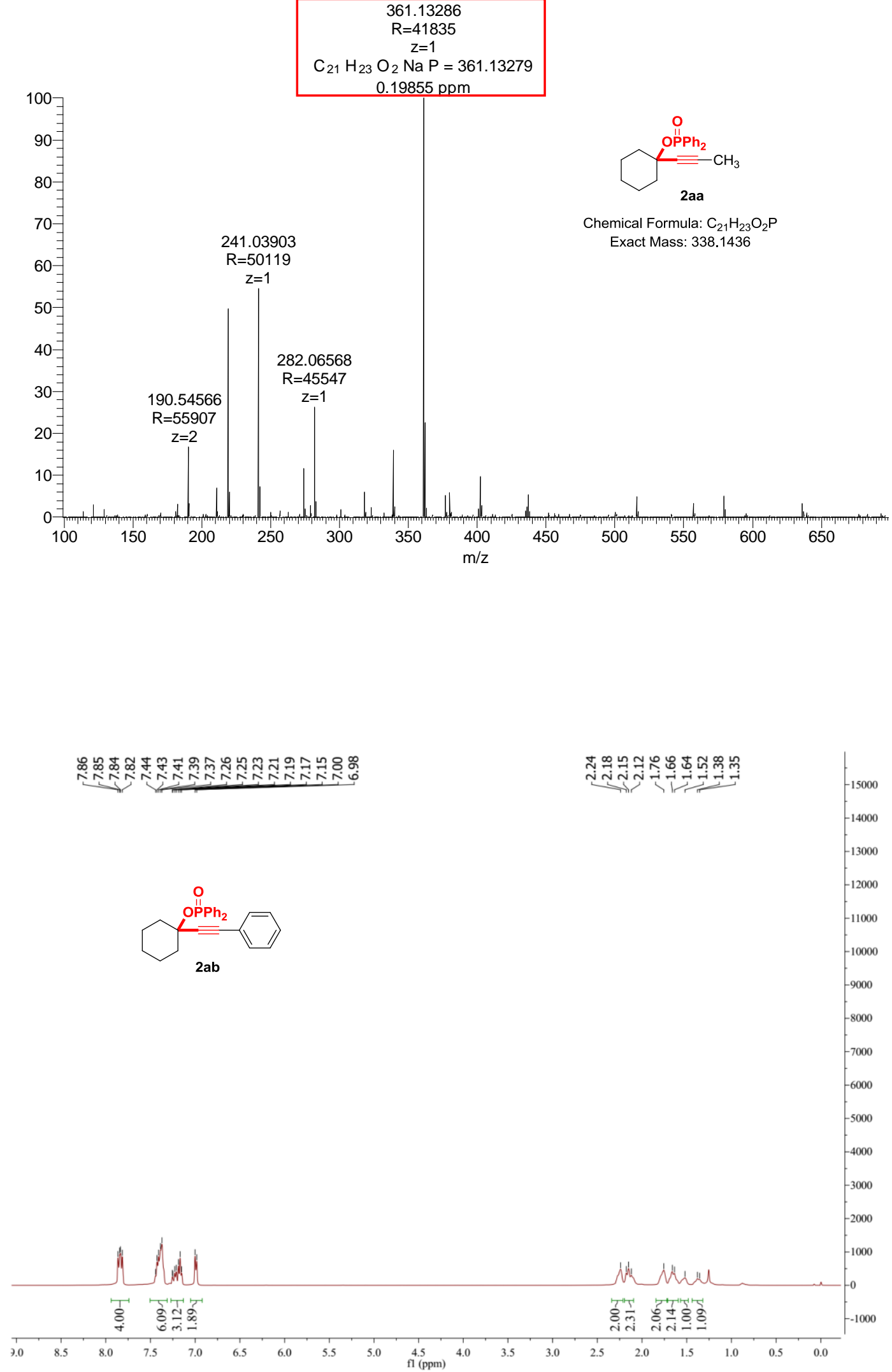

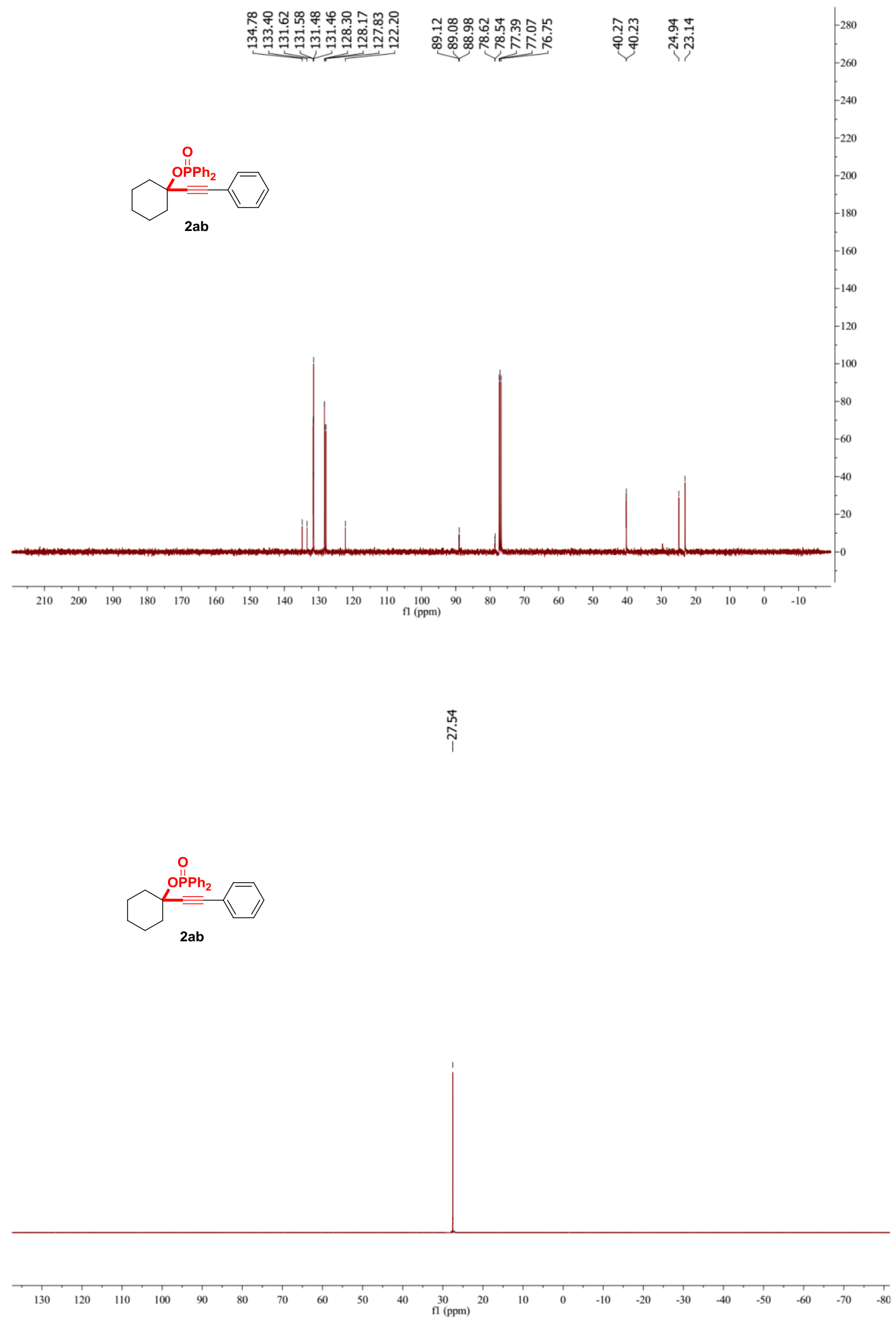
NJNY-20180507-1_180507161546 \#29 RT: 0.43 AV: 1 NL: 3.48E4 T: FTMS $\{1,1\}+p \overline{E S I}$ Full ms [100.00-1000.00]

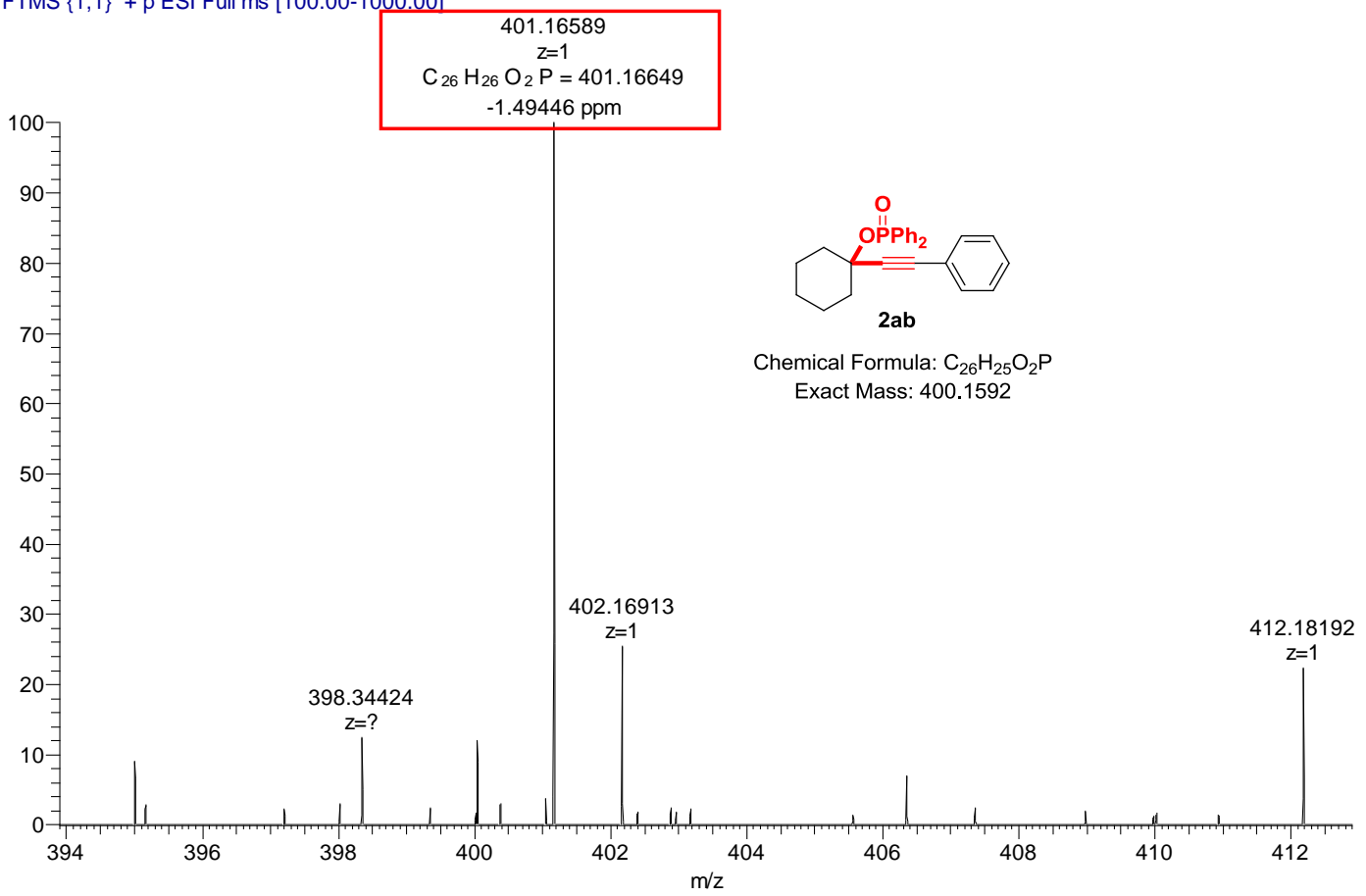

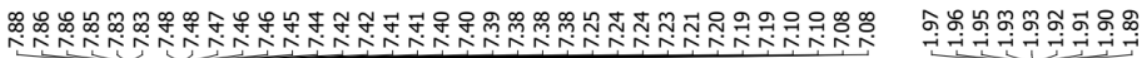

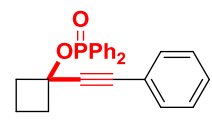

2ac

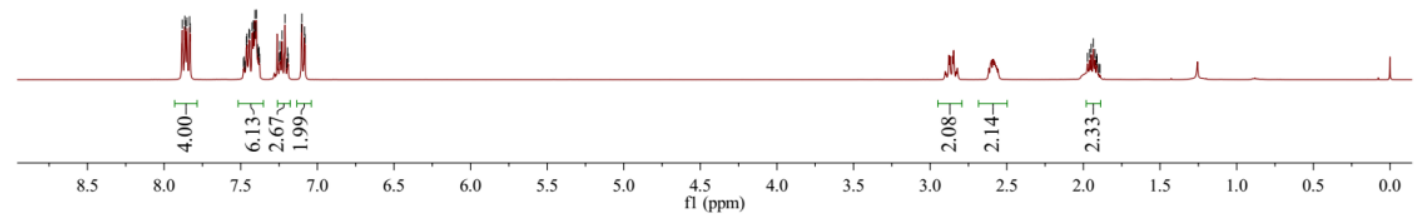




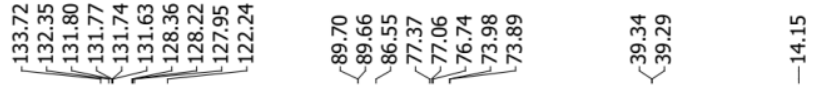
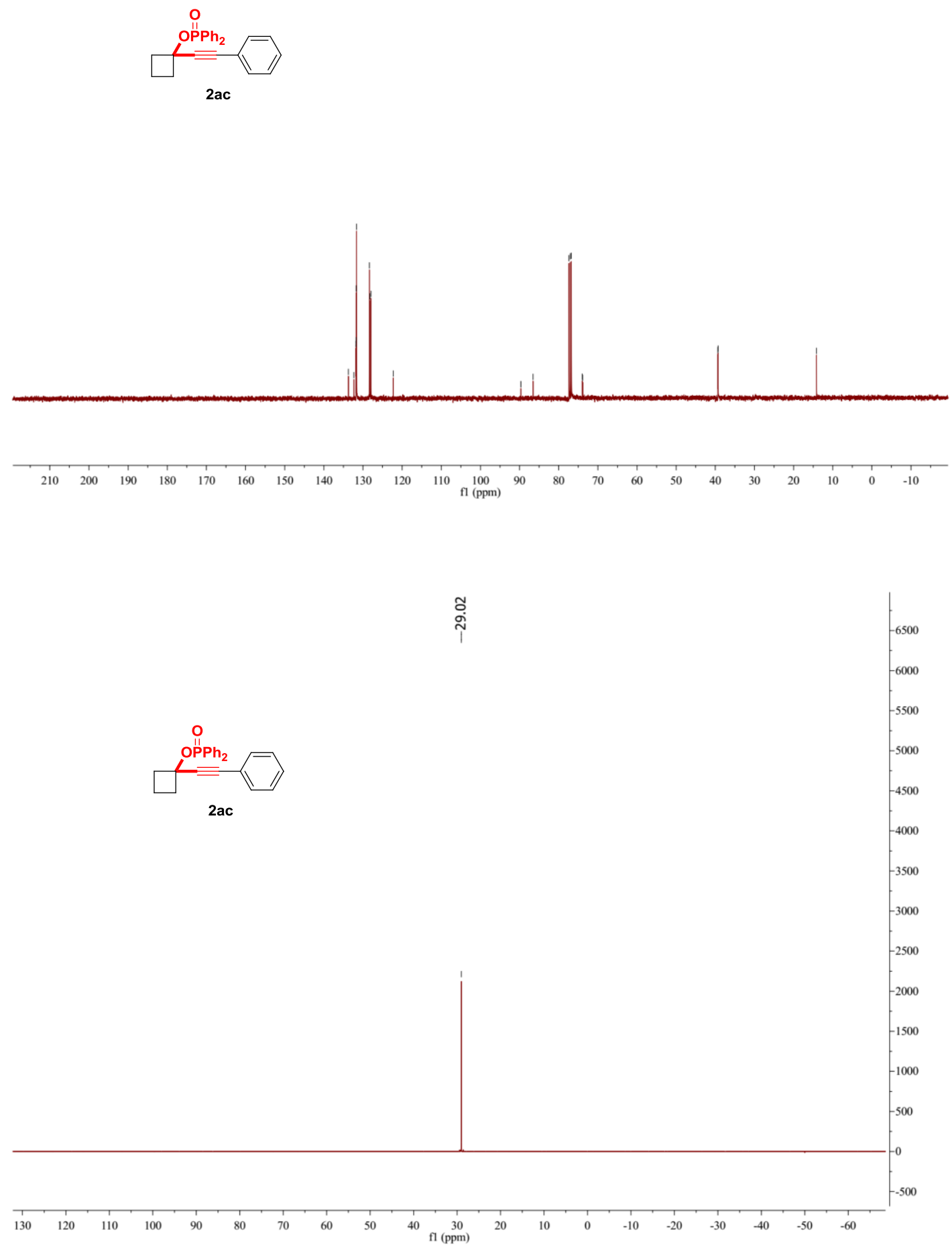
36 \#41 RT: 0.58 AV: 1 NL: $1.35 E 6$
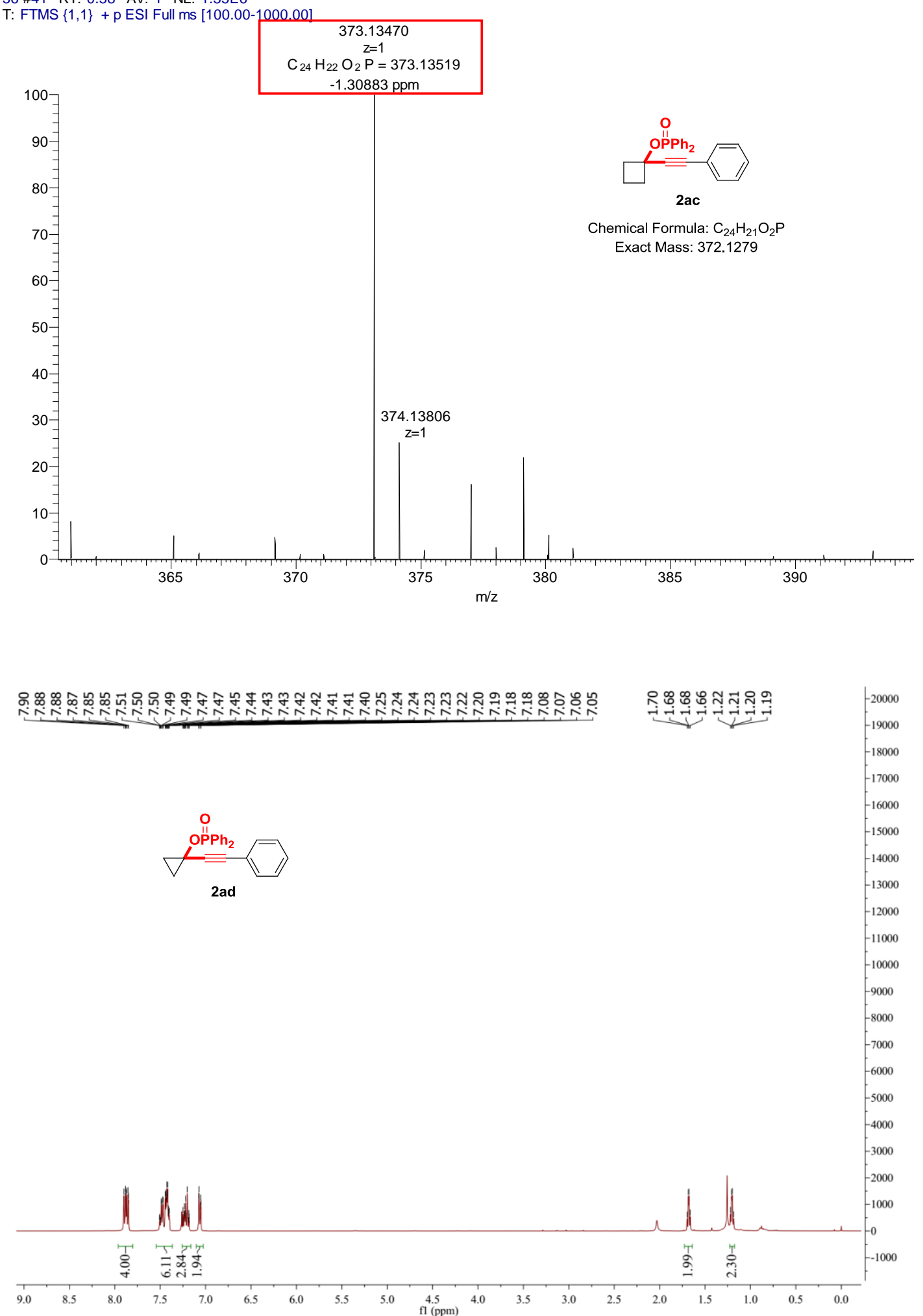

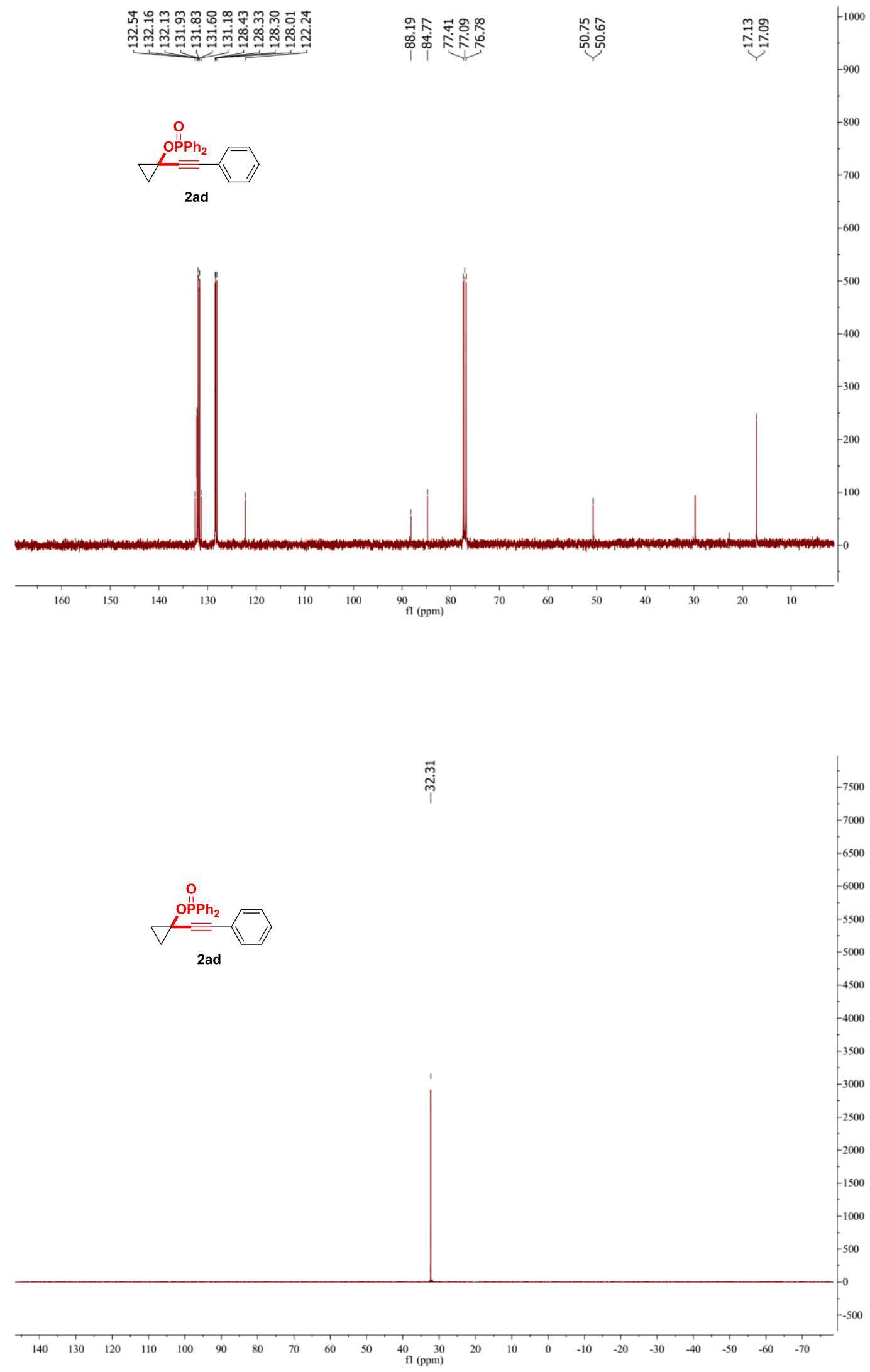
34 \#29 RT: 0.37 AV: 1 NL: $4.58 \mathrm{E} 7$

T: FTMS $\{1,1\}+p$ ESI Full ms [100.00-1000.00]

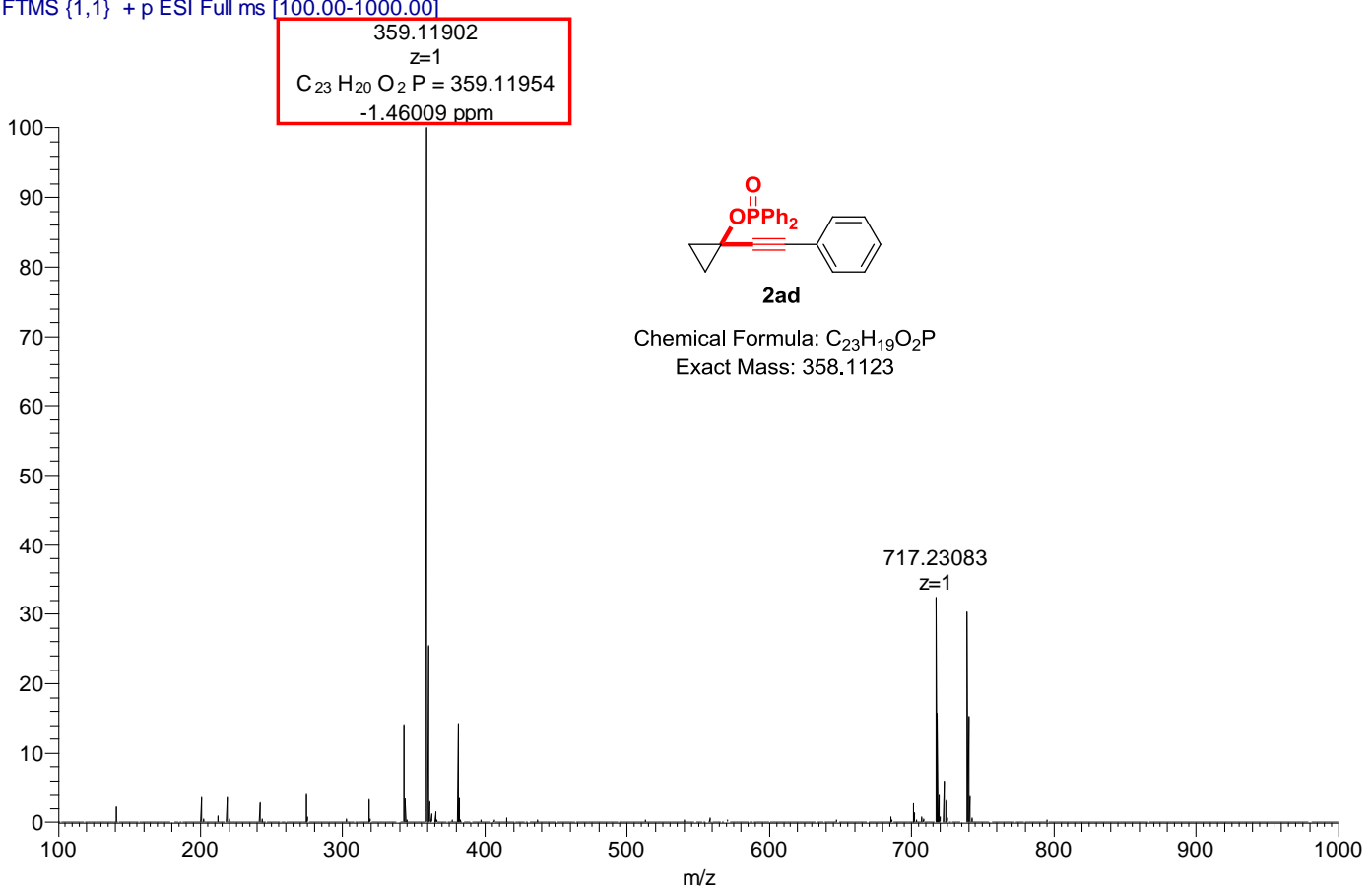

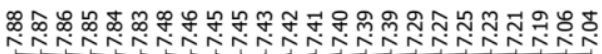

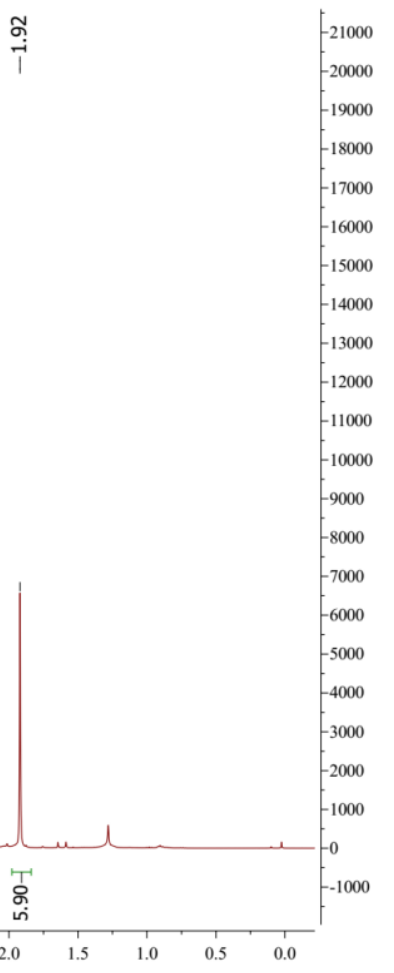



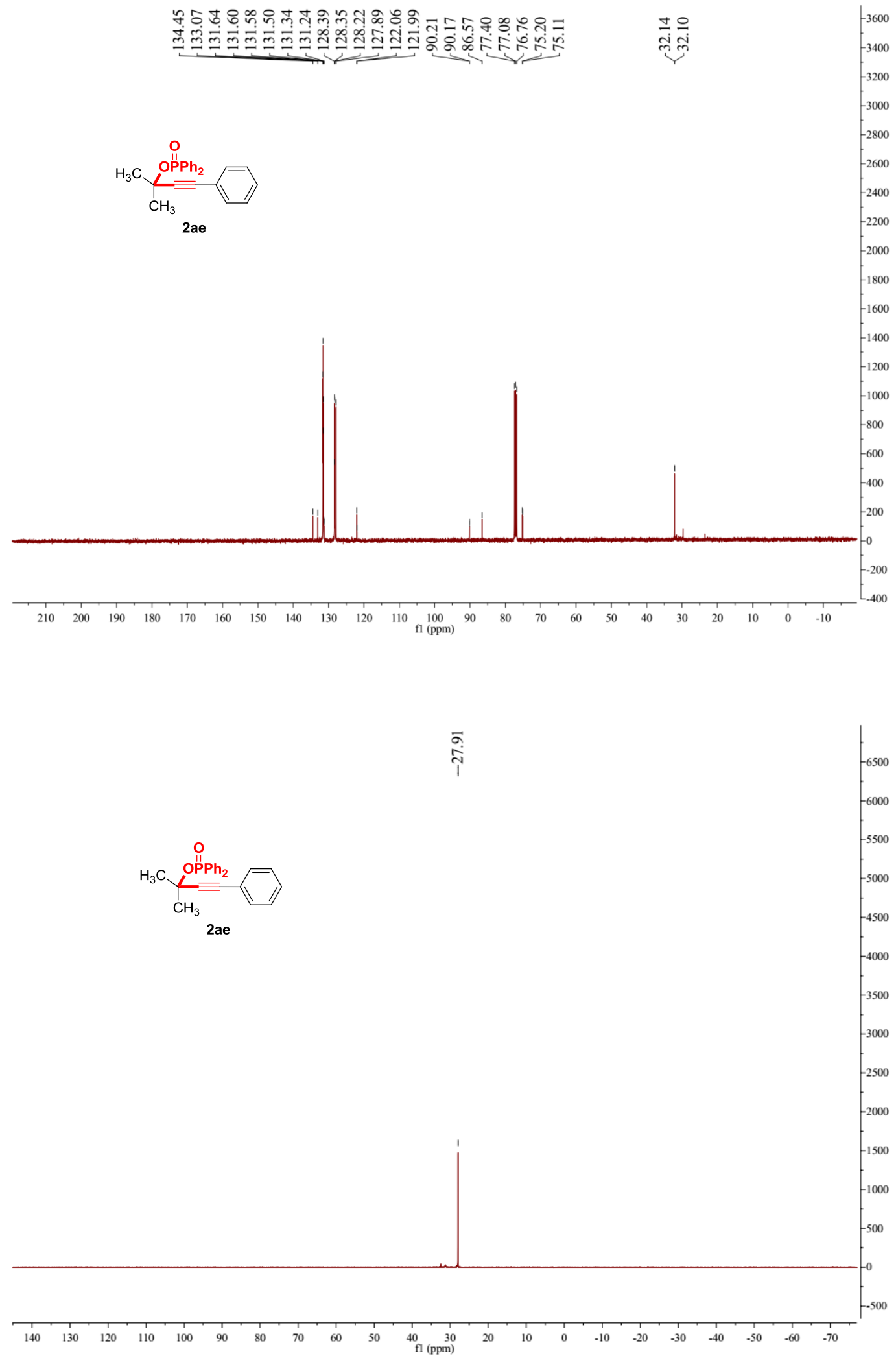
20170618-6 \#35-37 RT: 0.32-0.34 AV: 3 NL: 1.31E5

T: FTMS $\{1,1\}+p$ ESI Full ms [100.00-1000.00]

383.11676
$R=40478$

$\mathrm{z}=1$

$\mathrm{C}_{23} \mathrm{H}_{21} \mathrm{O}_{2} \mathrm{NaP}=383.11714$

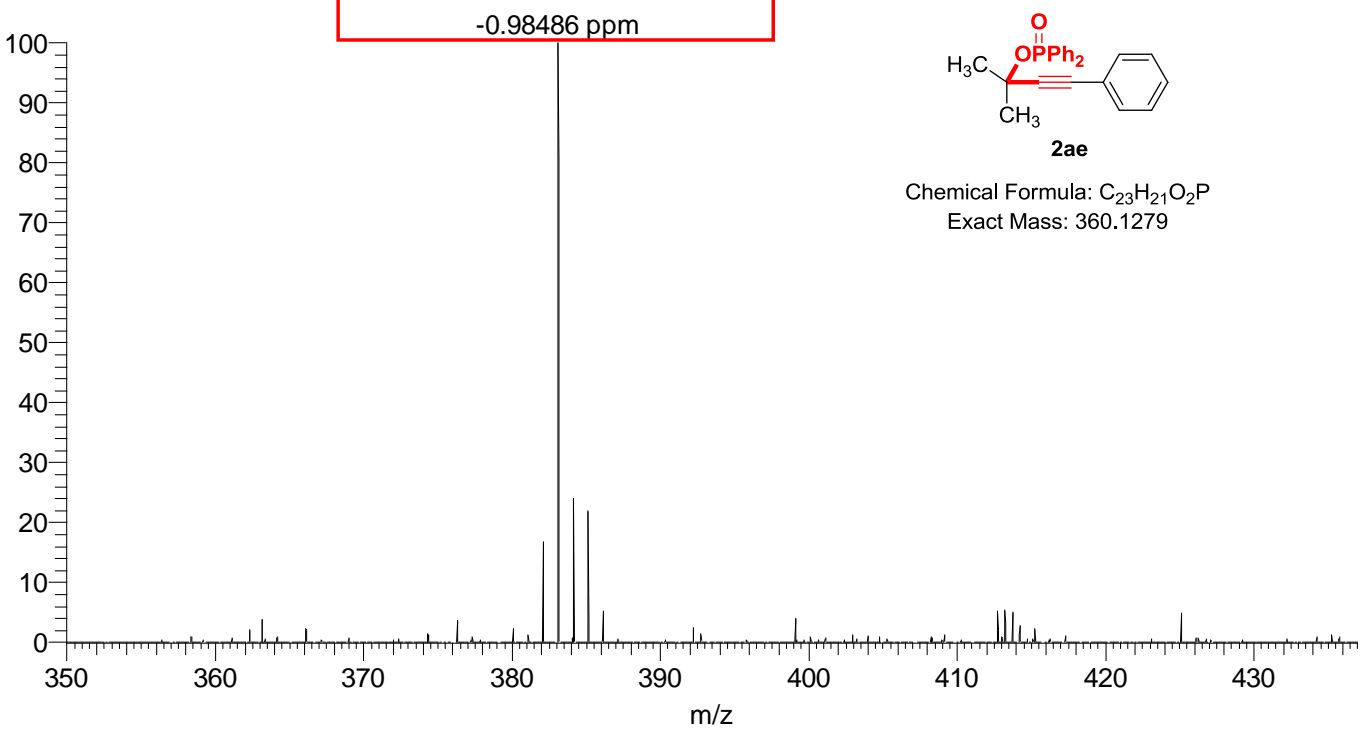
som

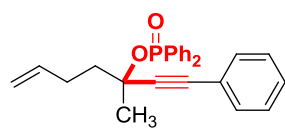

2af

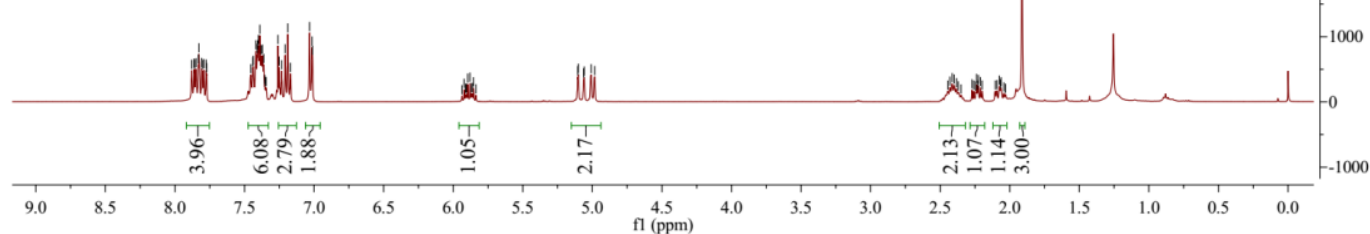



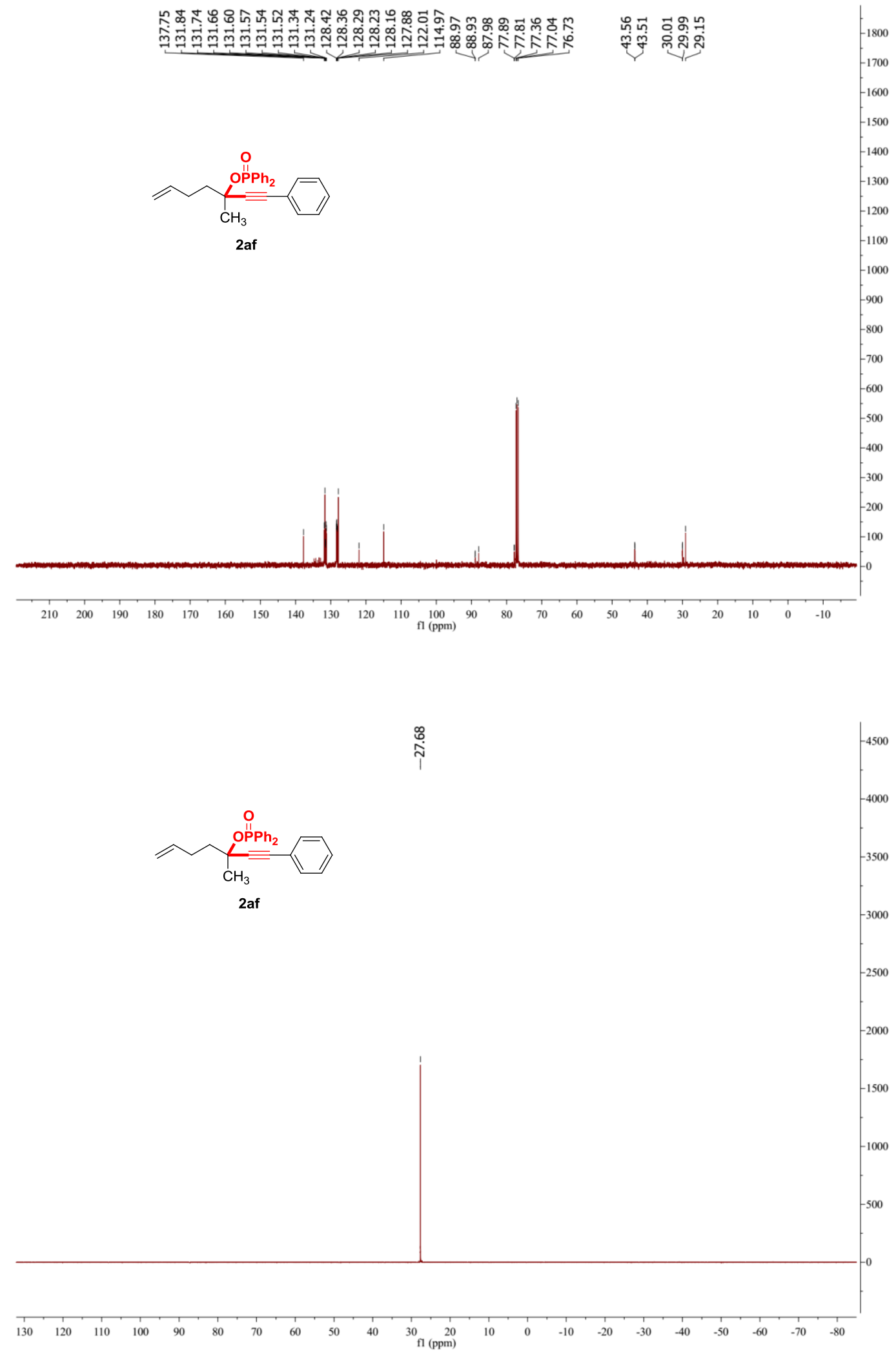
NJNY-20180507-2_180507162122 \#31 RT: 0.43 AV: 1 NL: 9.82E4 T: FTMS $\{1,1\}+$ p ESI Full ms [100.00-1000.00]

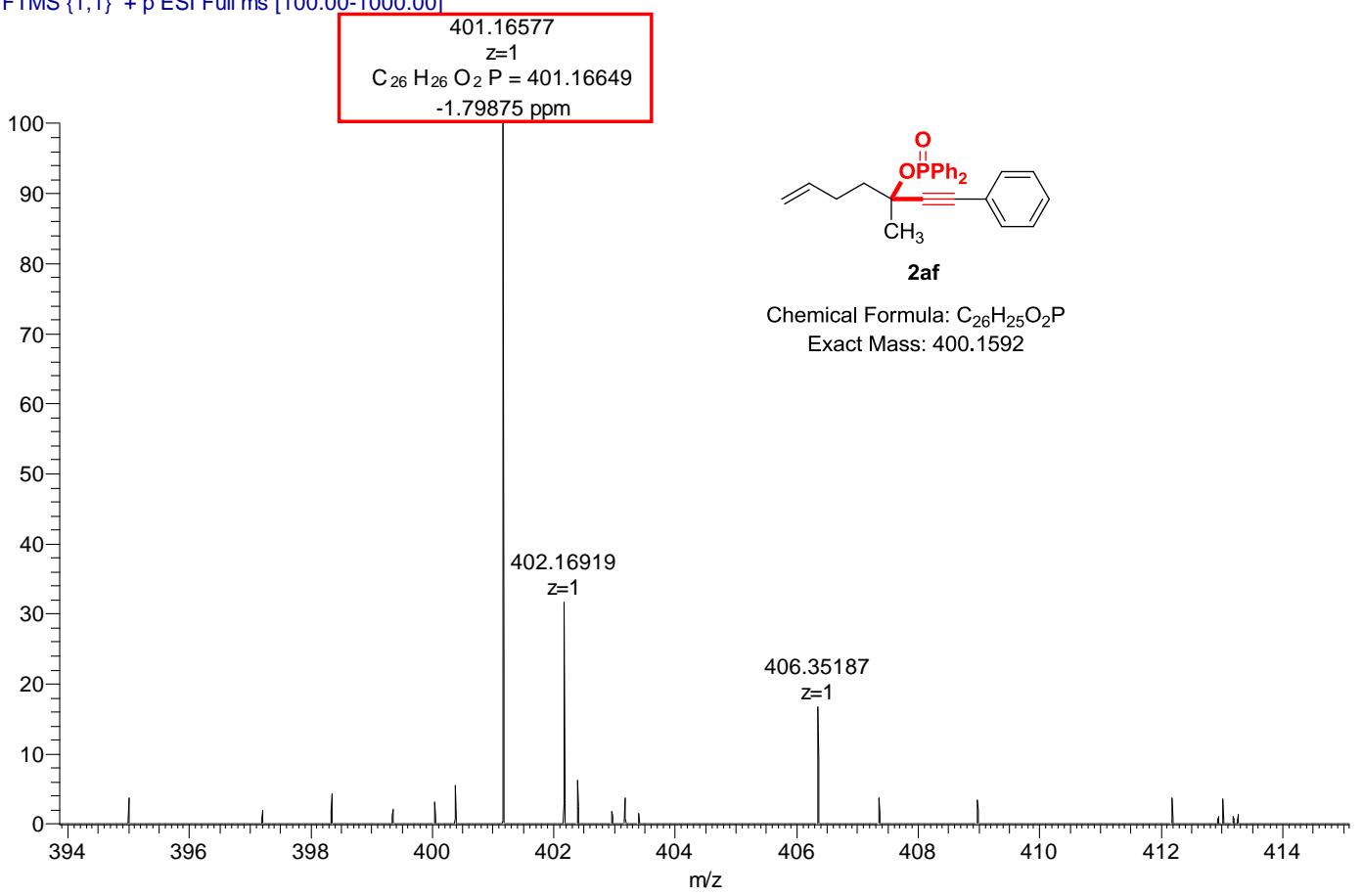

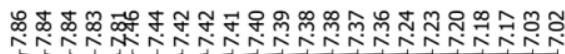<smiles></smiles>

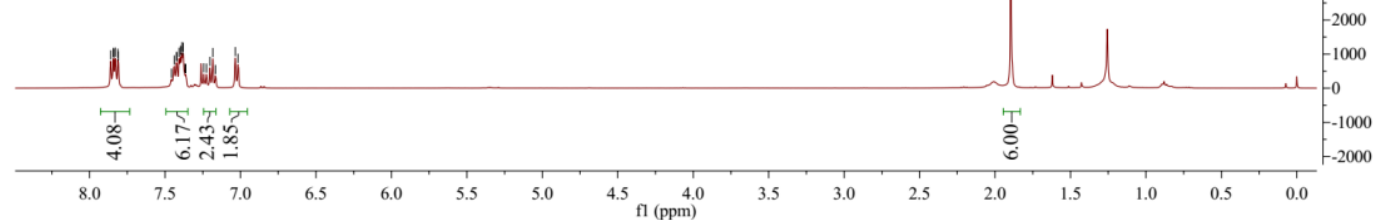



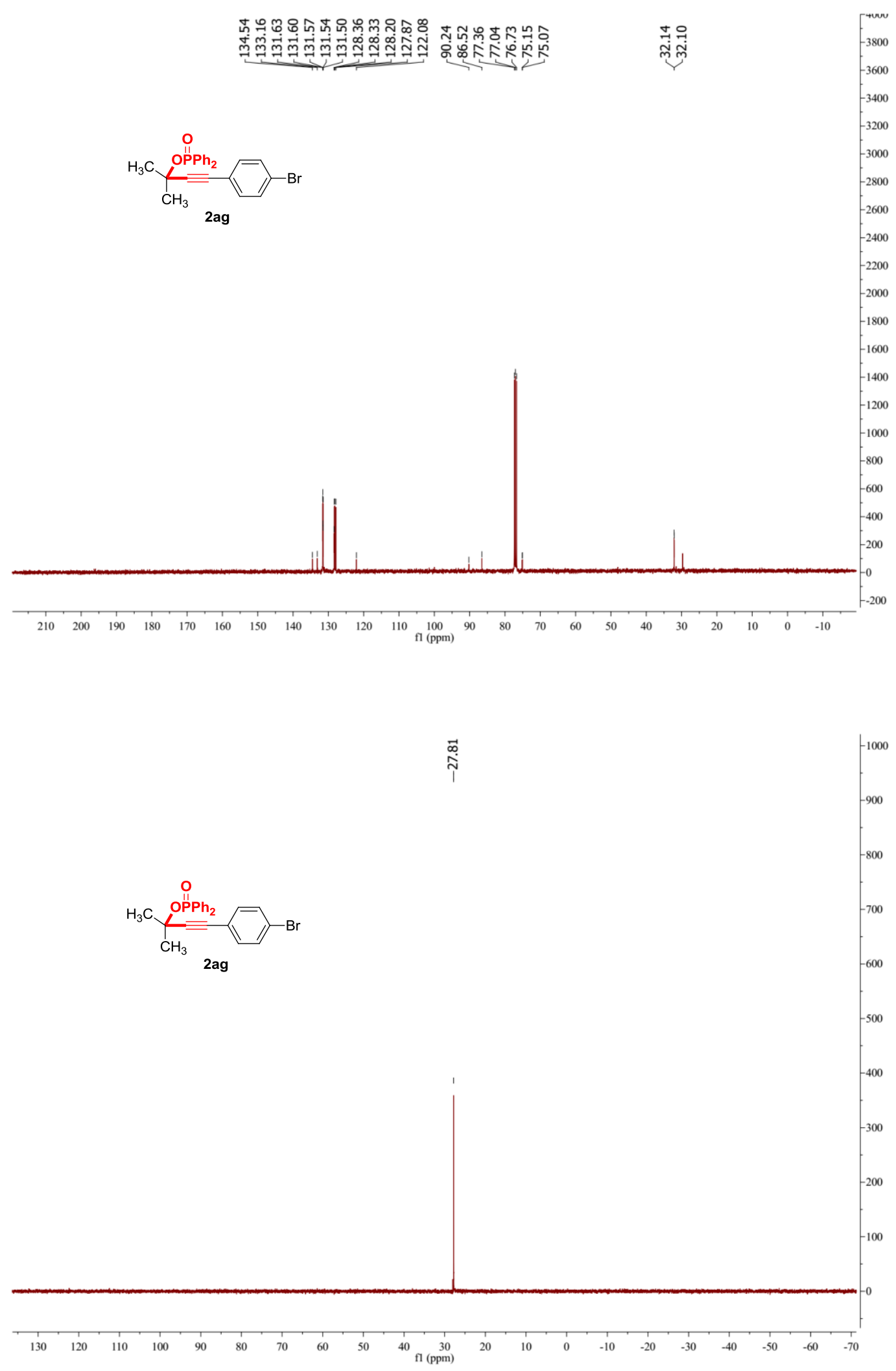

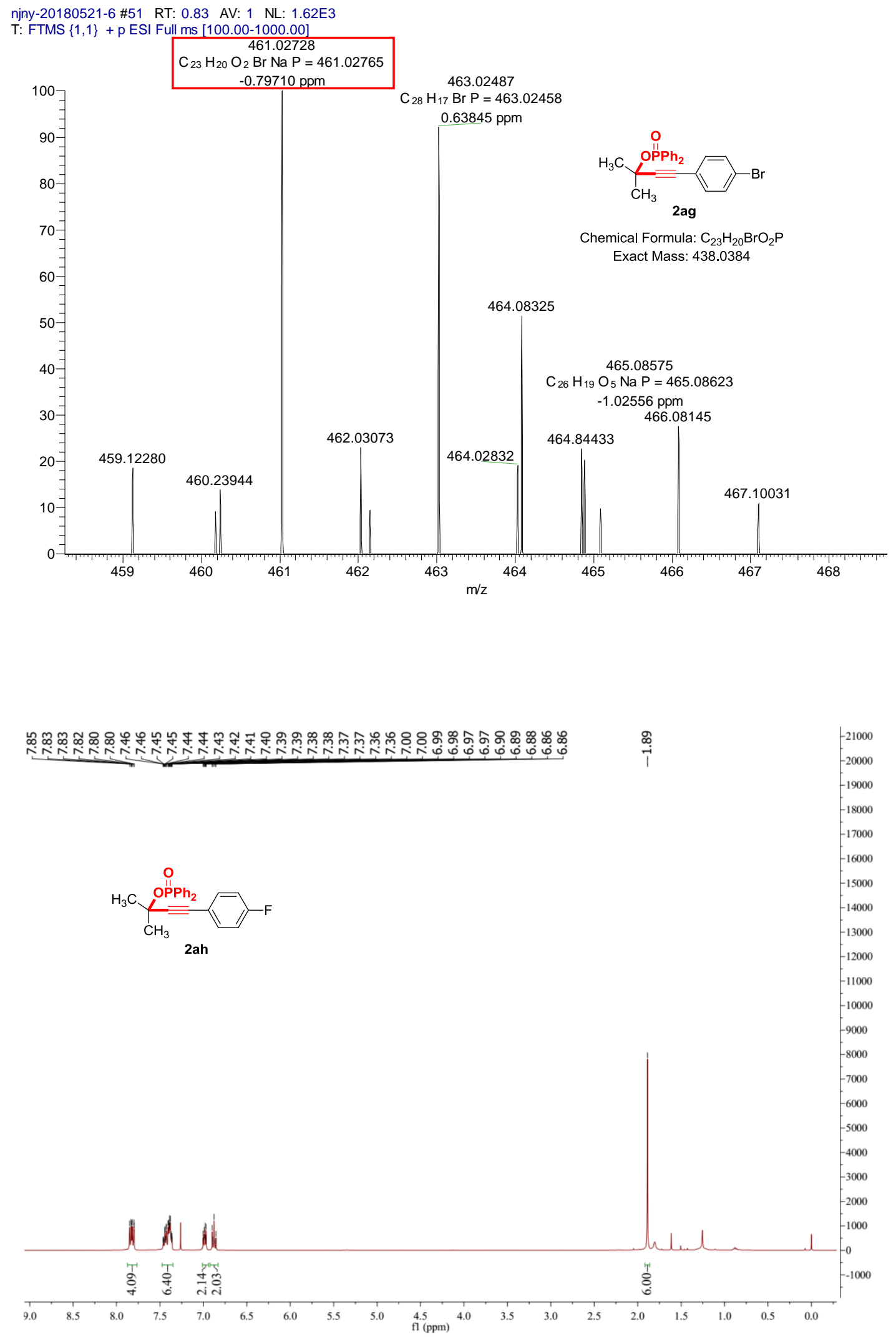

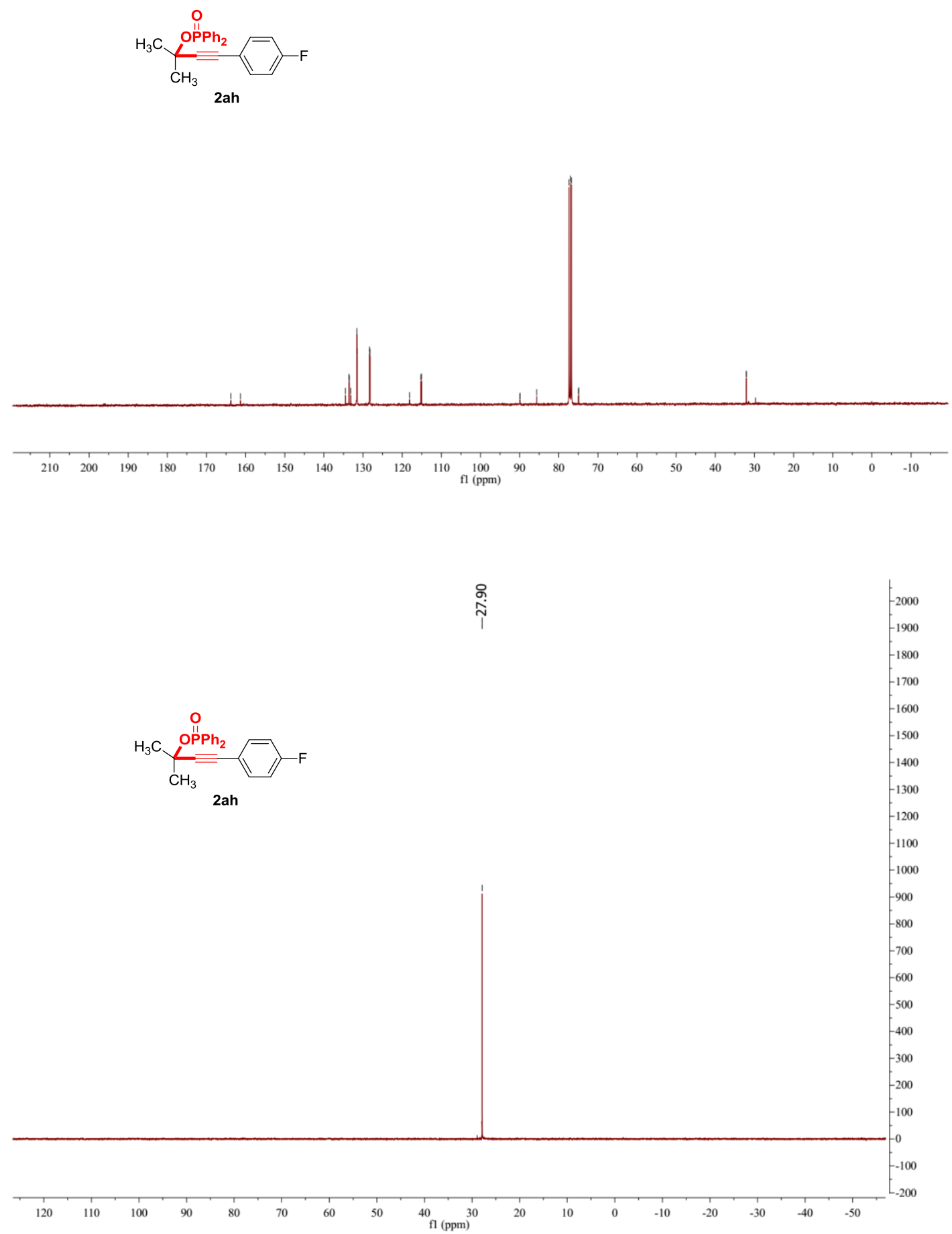


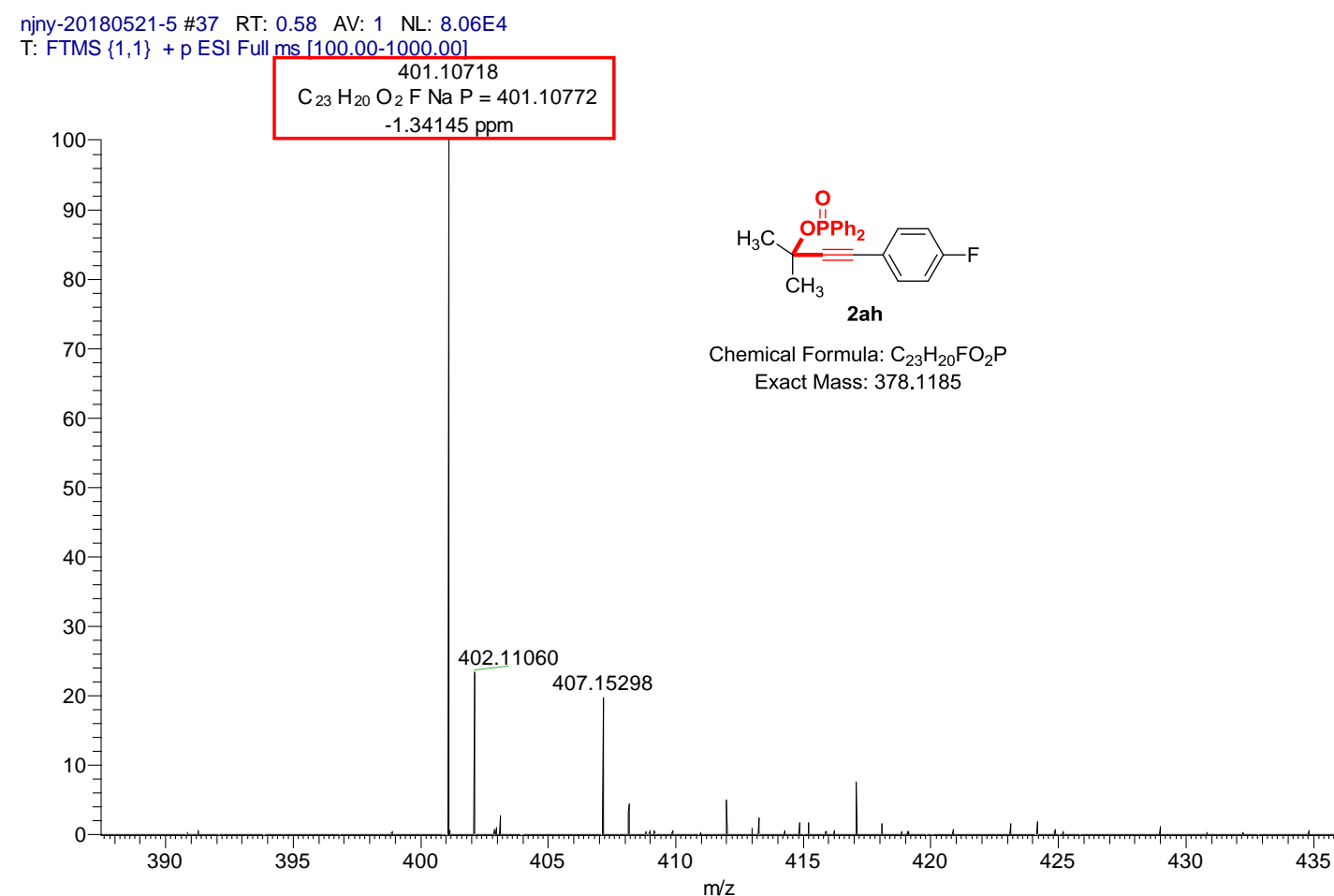

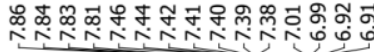

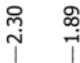

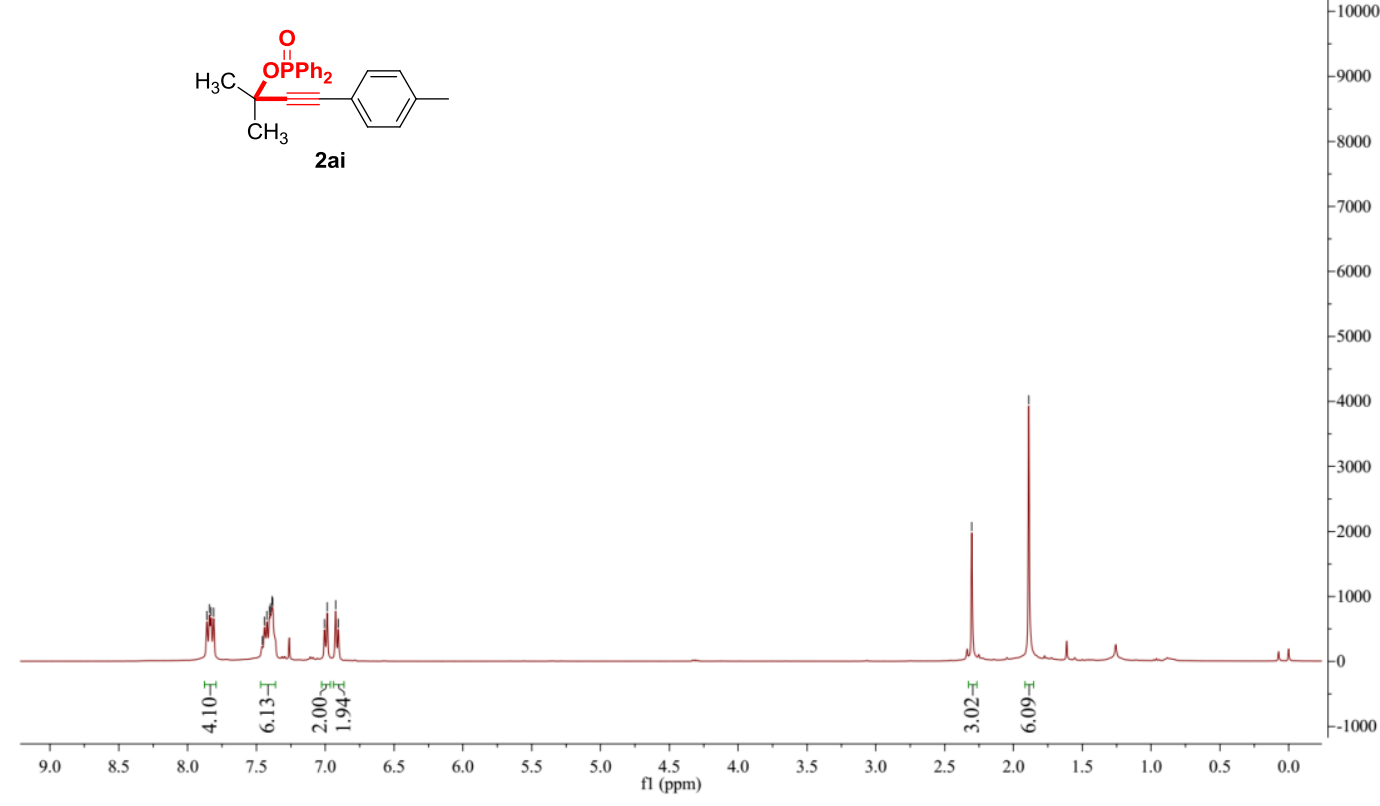



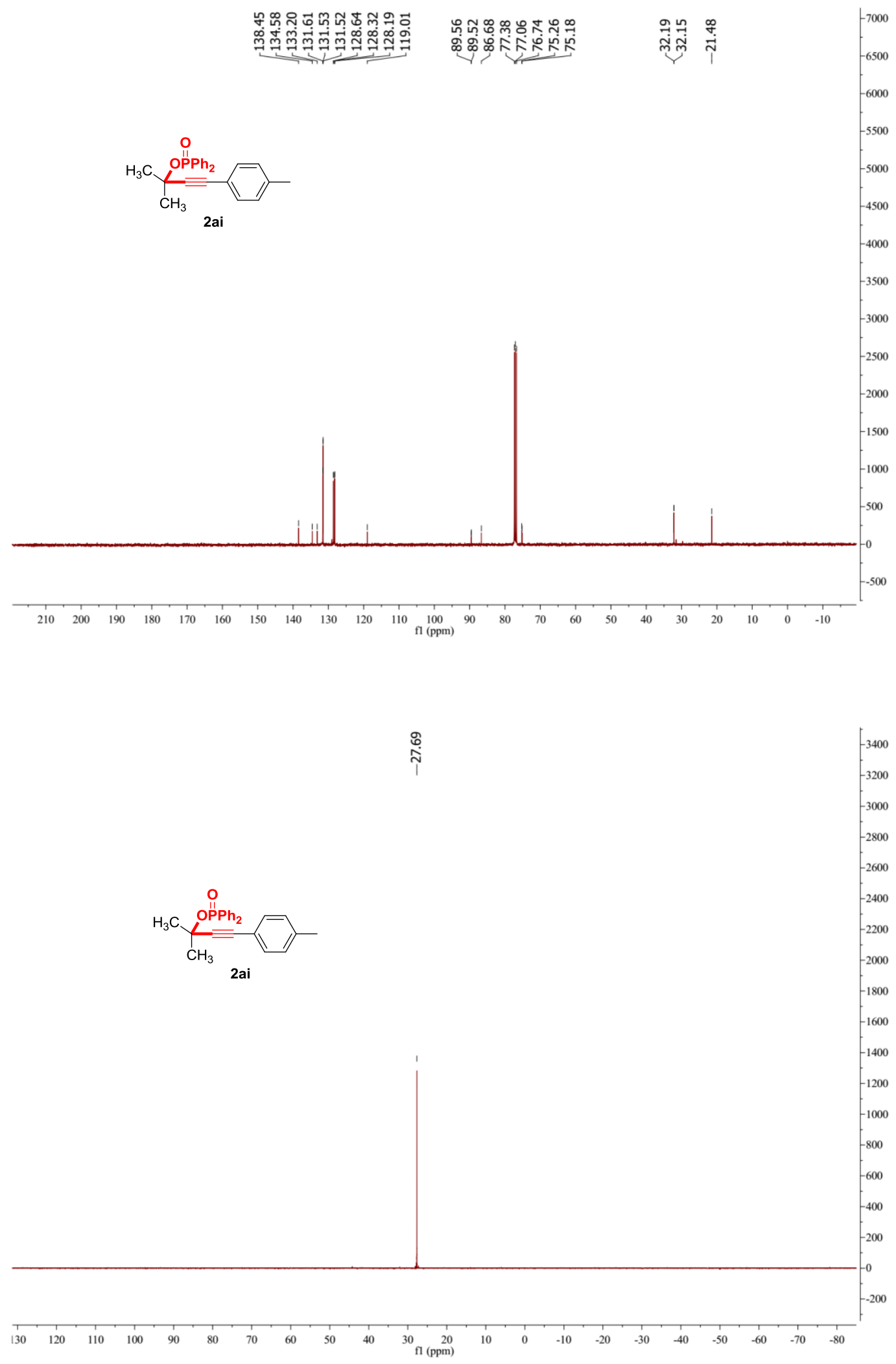
njny-20180521-11 \#39 RT: 0.53 AV: 1 NL: 1.12E5
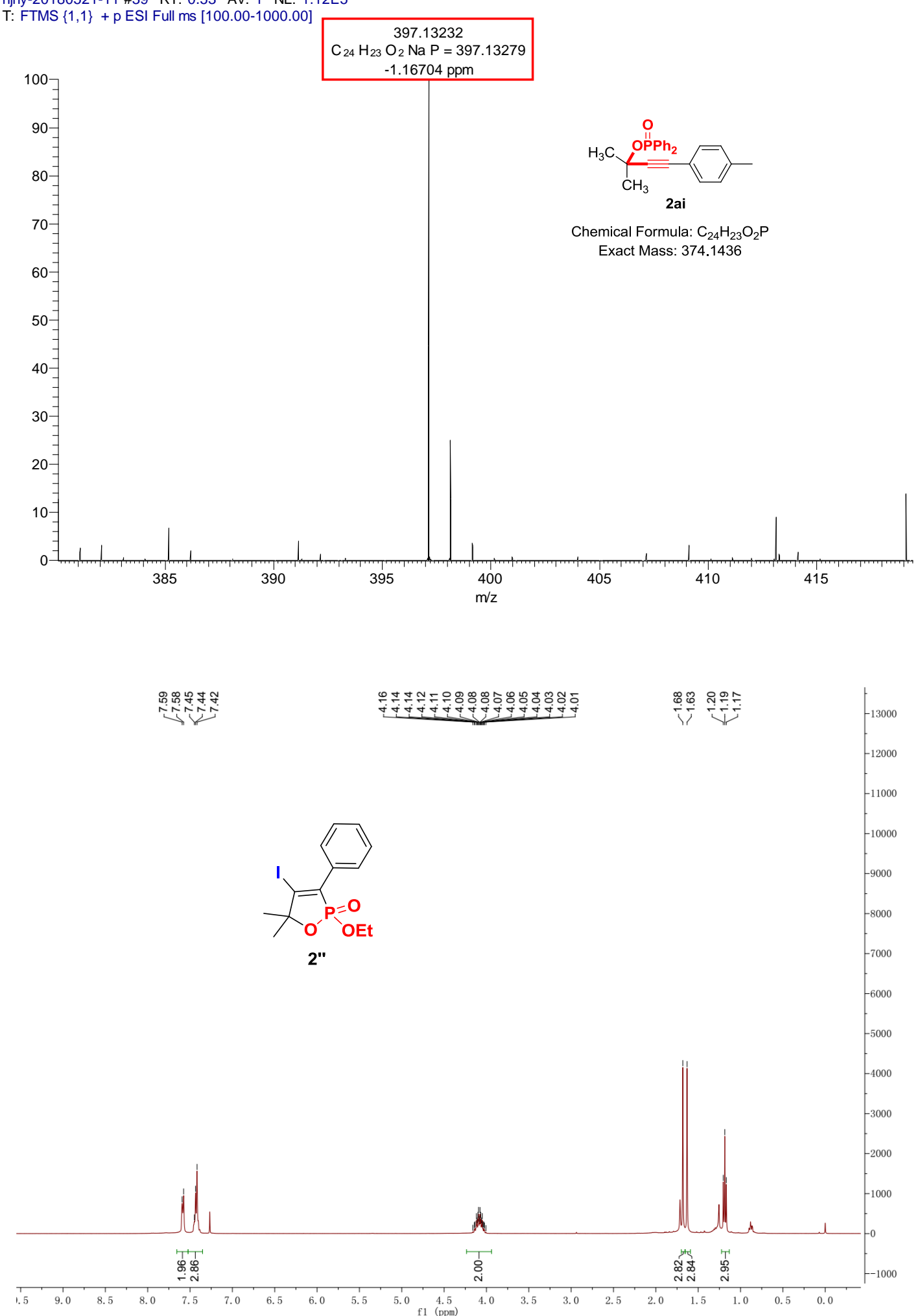


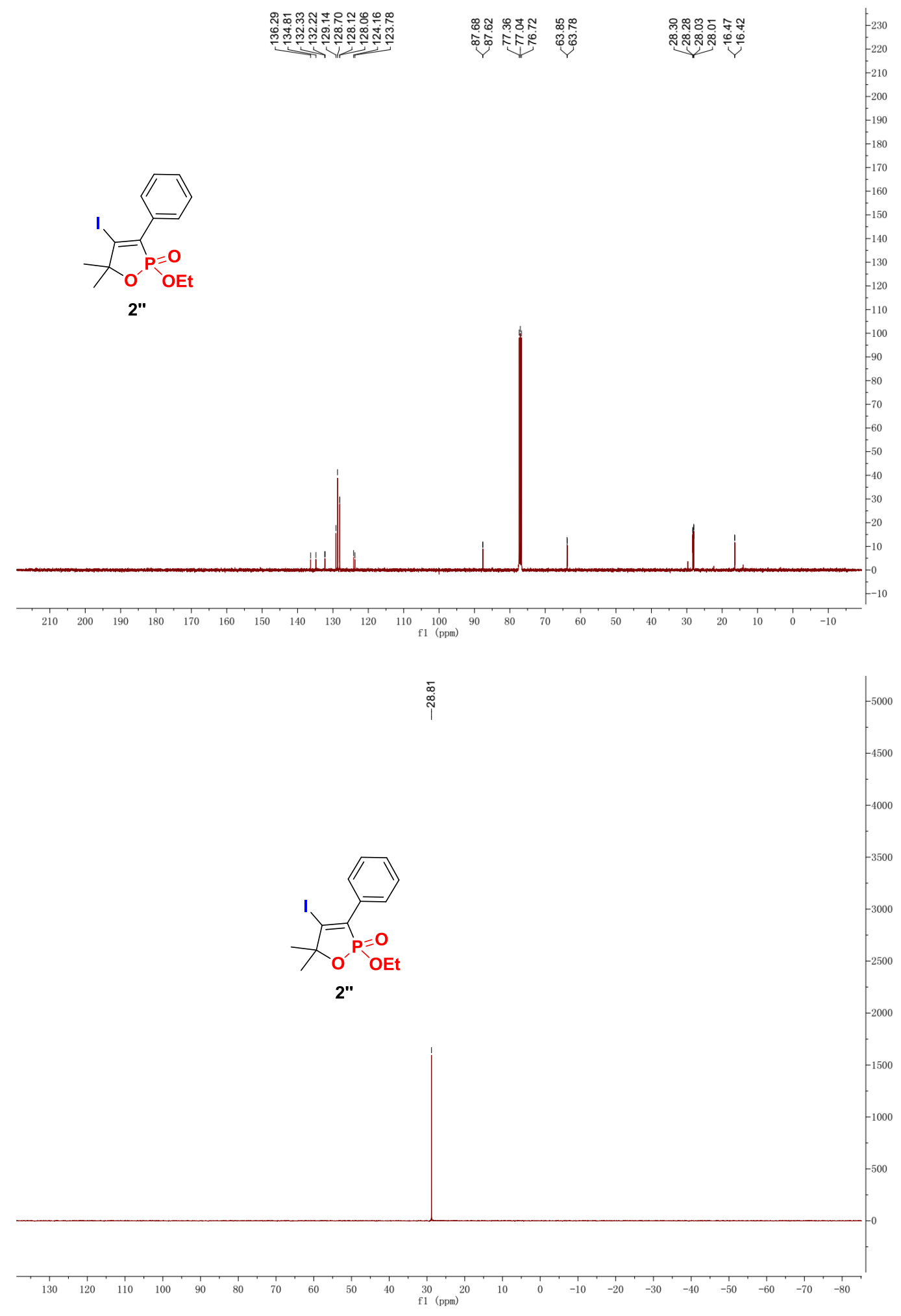




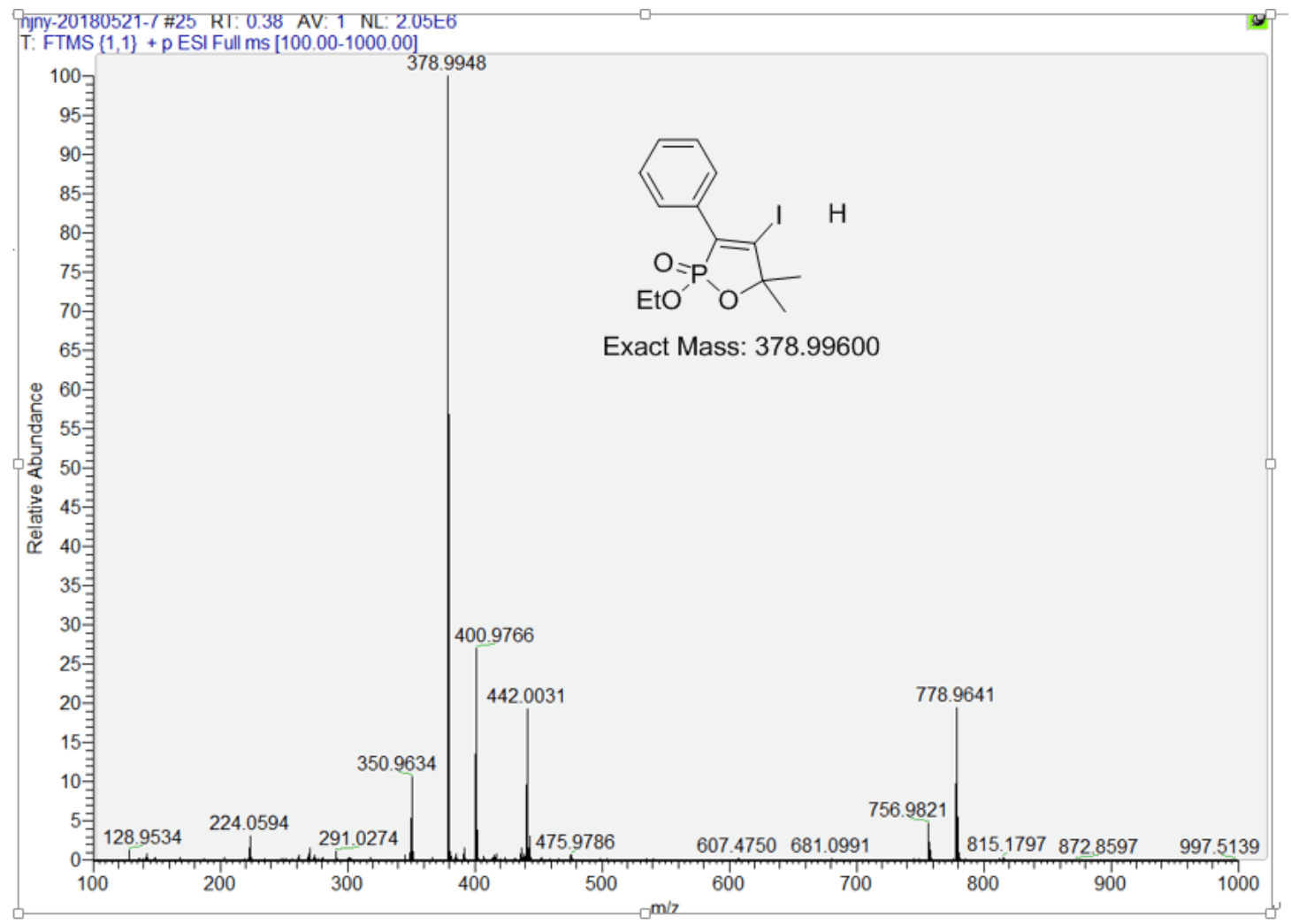

